\title{
Biomimetic Total Synthesis of Dysoxylum Alkaloids
}

\author{
Carlos E. Puerto Galvis, and Vladimir V. Kouznetsov* \\ Laboratorio de Química Orgánica y Biomolecular, CMN, Universidad Industrial de Santander. Parque \\ Tecnológico Guatiguará, Km 2 Vía Refugio, Piedecuesta 681011, Colombia. \\ *kouznet@uis.edu.co;vkuznechnik@gmail.com
}

\section{ELECTRONIC SUPPORTING INFORMATION}

List of contents

1. Copies of ${ }^{1} \mathrm{H}$ NMR, ${ }^{13} \mathrm{C}$ NMR and DEPT-135 charts of zanthoxylamide protoalkaloids 3a-i

2. Copies of ${ }^{1} \mathrm{H}$ NMR, ${ }^{13} \mathrm{C}$ NMR and DEPT-135 charts of $N$-phenethyl-3phenylpropanamides $4 \mathbf{a}-\mathbf{h}$

3. Copies of ${ }^{1} \mathrm{H}$ NMR, ${ }^{13} \mathrm{C}$ NMR and DEPT-135 charts of 1-phenethyl3,4-dihydroisoquinolines 5a-i

4. Copies of ${ }^{1} \mathrm{H}$ NMR, ${ }^{13} \mathrm{C}$ NMR and DEPT-135 charts of $(R)-7 \mathrm{a}-\mathrm{i}$ and (S)-7a-i Dysoxylum alkaloids

5. Copies of chiral HPLC Chromatograms of $(R)-7 \mathrm{a}-\mathrm{i}$ and $(S)-7 \mathrm{a}-\mathrm{i}$ Dysoxylum alkaloids
Pages

SI-2

SI-15

SI-25

SI-37

SI-64 


\section{Copies of ${ }^{1} \mathrm{H}$ NMR, ${ }^{13} \mathrm{C}$ NMR and DEPT-135 charts of zanthoxylamide protoalkaloids 3a-i}

Figure S1. ${ }^{1} \mathrm{H}-\mathrm{NMR}$ spectrum of $N$-(3,4-dimethoxyphenylethyl) cinnamamide (3a).

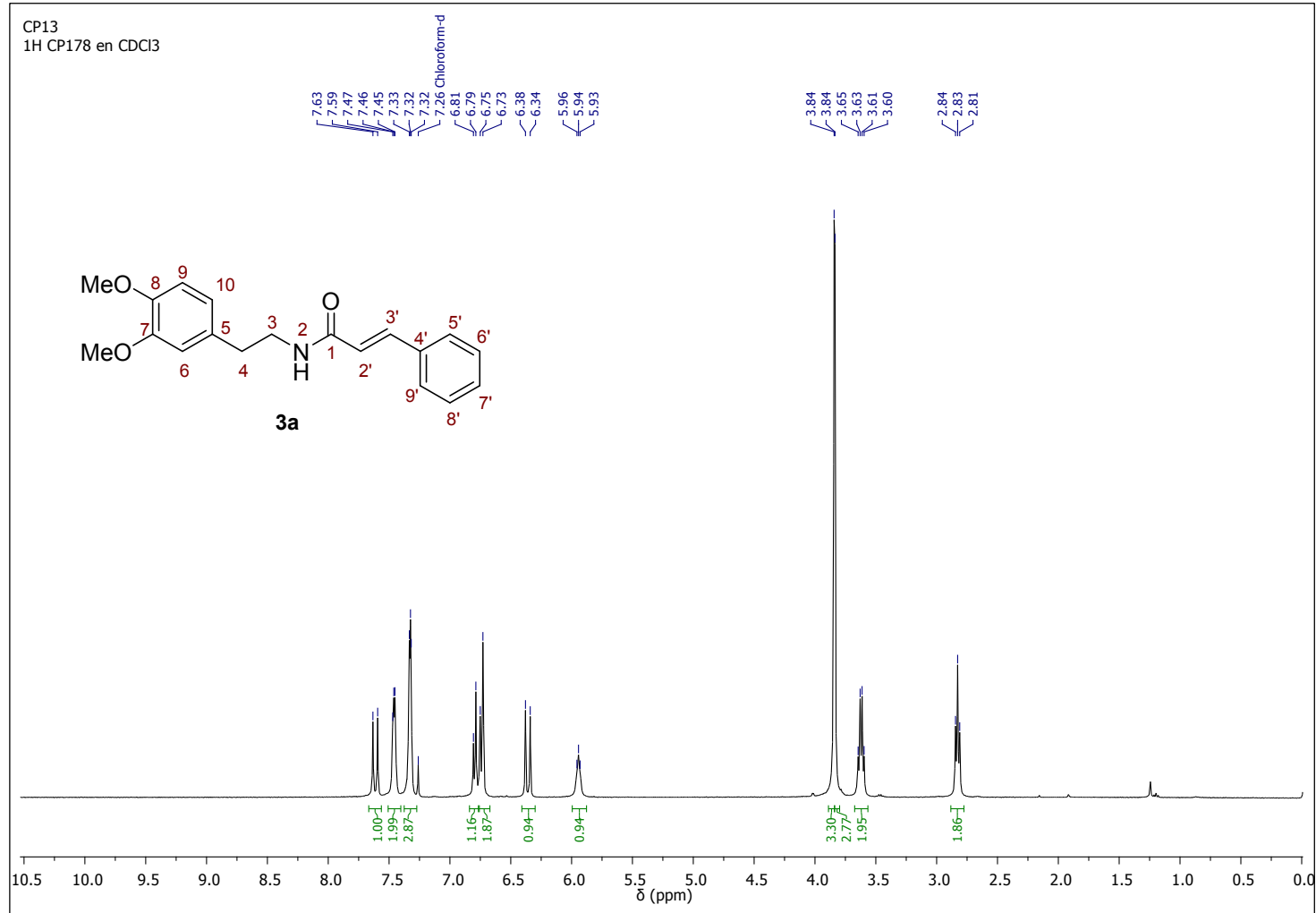

Figure S2. ${ }^{13} \mathrm{C}-\mathrm{NMR}$ spectrum of $N$-(3,4-dimethoxyphenylethyl) cinnamamide (3a).

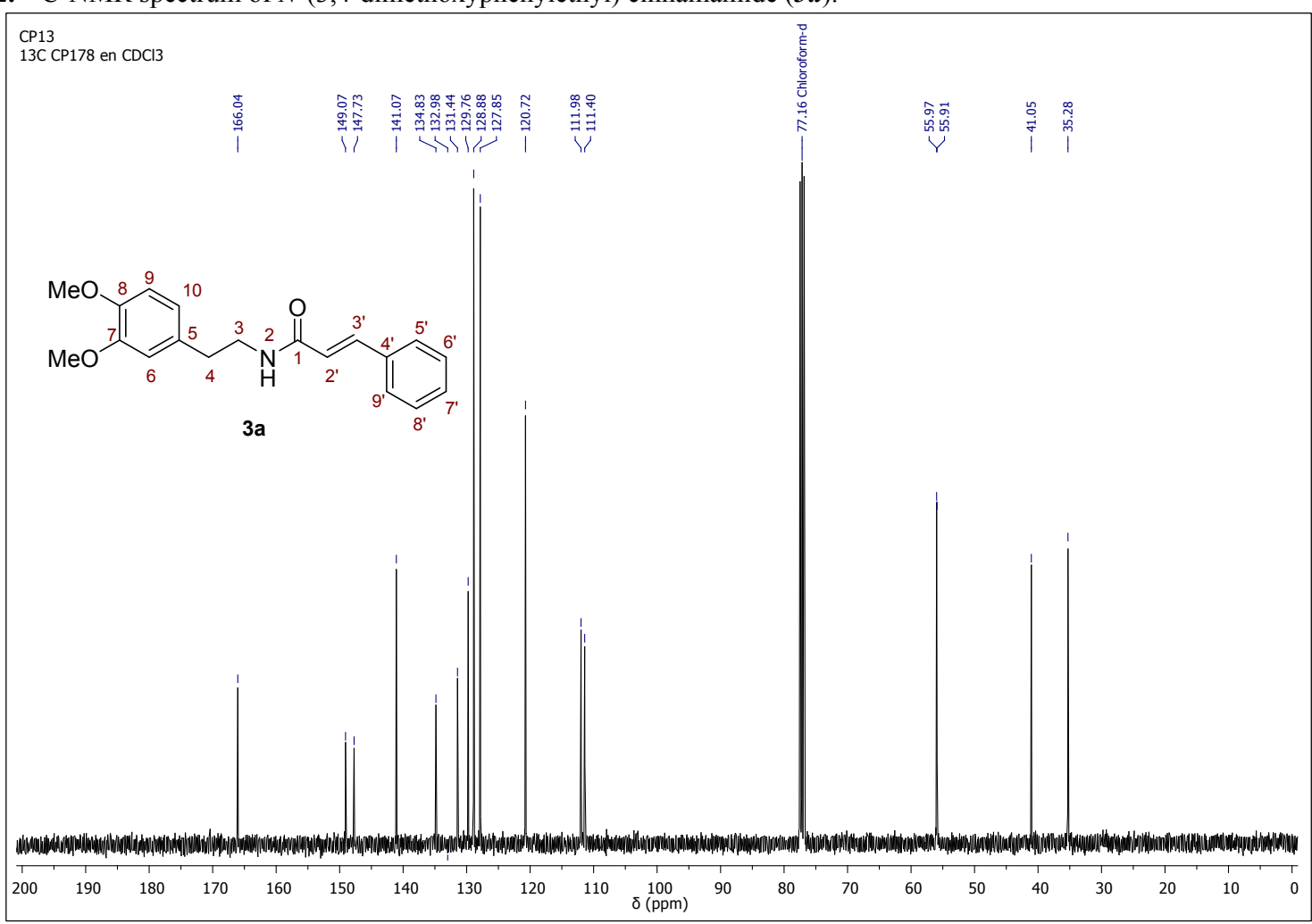


Figure S3. DEPT-135 spectrum of $N$-(3,4-dimethoxyphenylethyl) cinnamamide (3a).

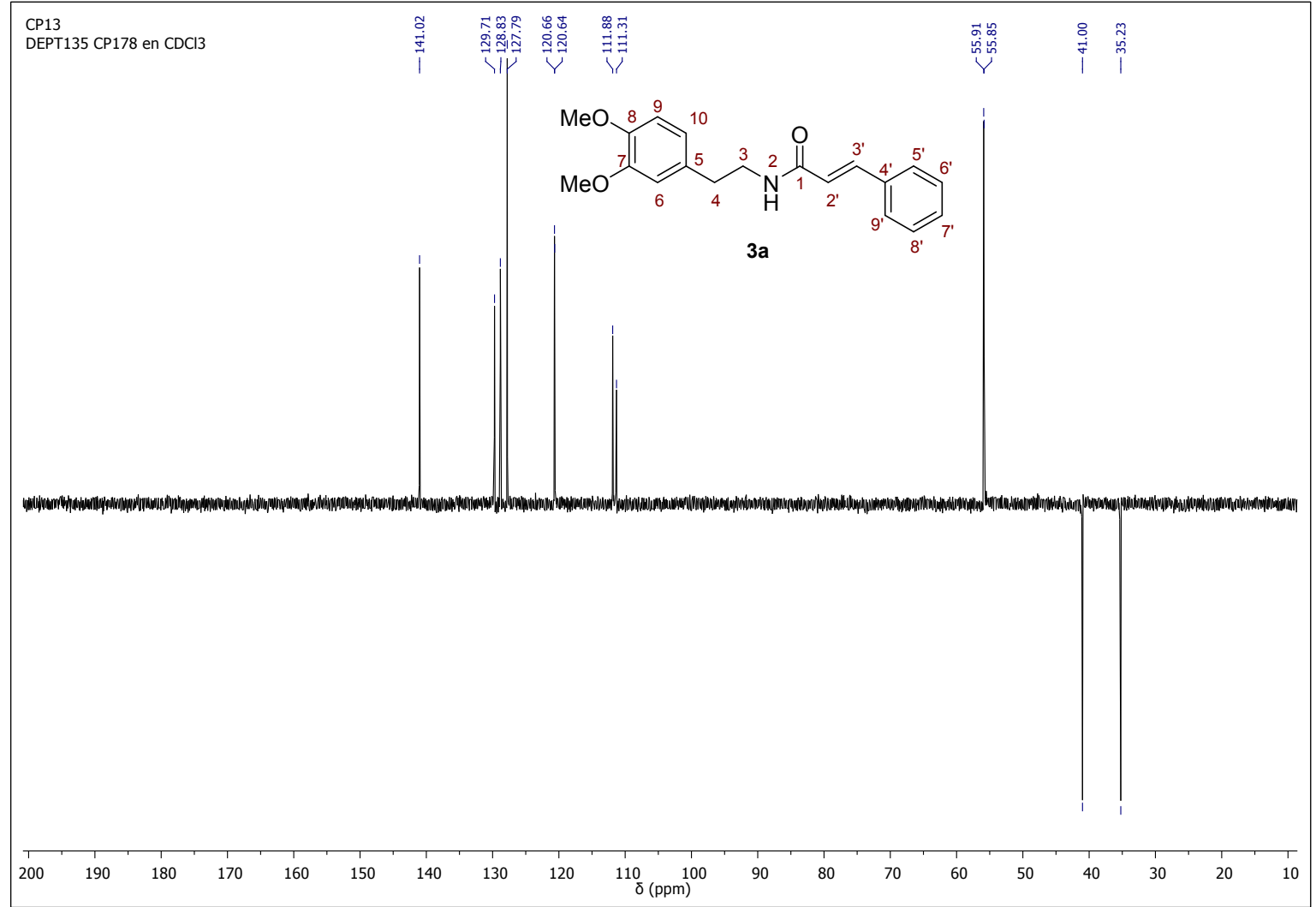

Figure S4. ${ }^{1} \mathrm{H}-\mathrm{NMR}$ spectrum of $N$-(3,4-dimethoxyphenethyl)-3-(4-methoxyphenyl)propanamide (3b).

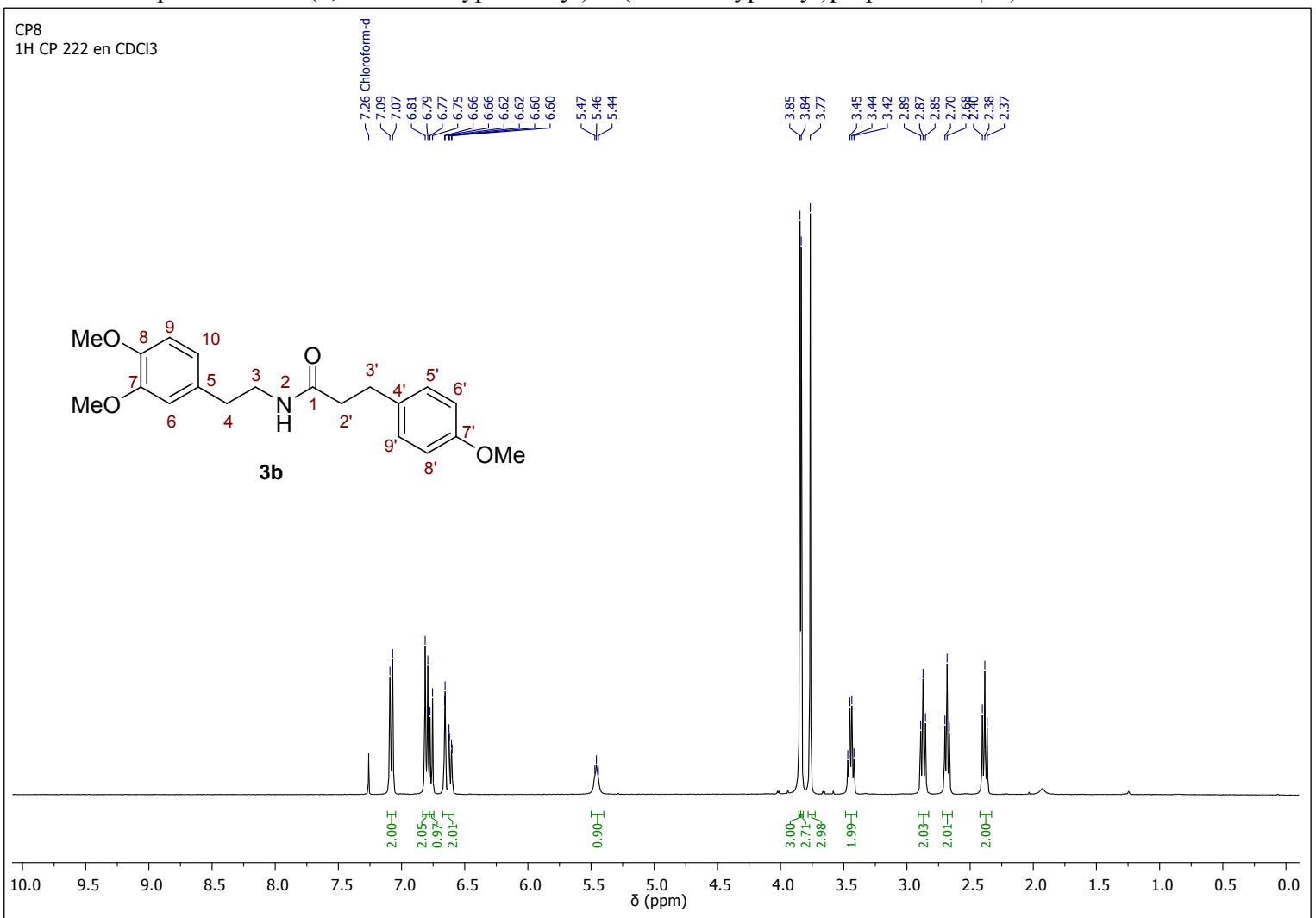


Figure S5. ${ }^{13} \mathrm{C}-\mathrm{NMR}$ spectrum of $N$-(3,4-dimethoxyphenethyl)-3-(4-methoxyphenyl)propanamide (3b).

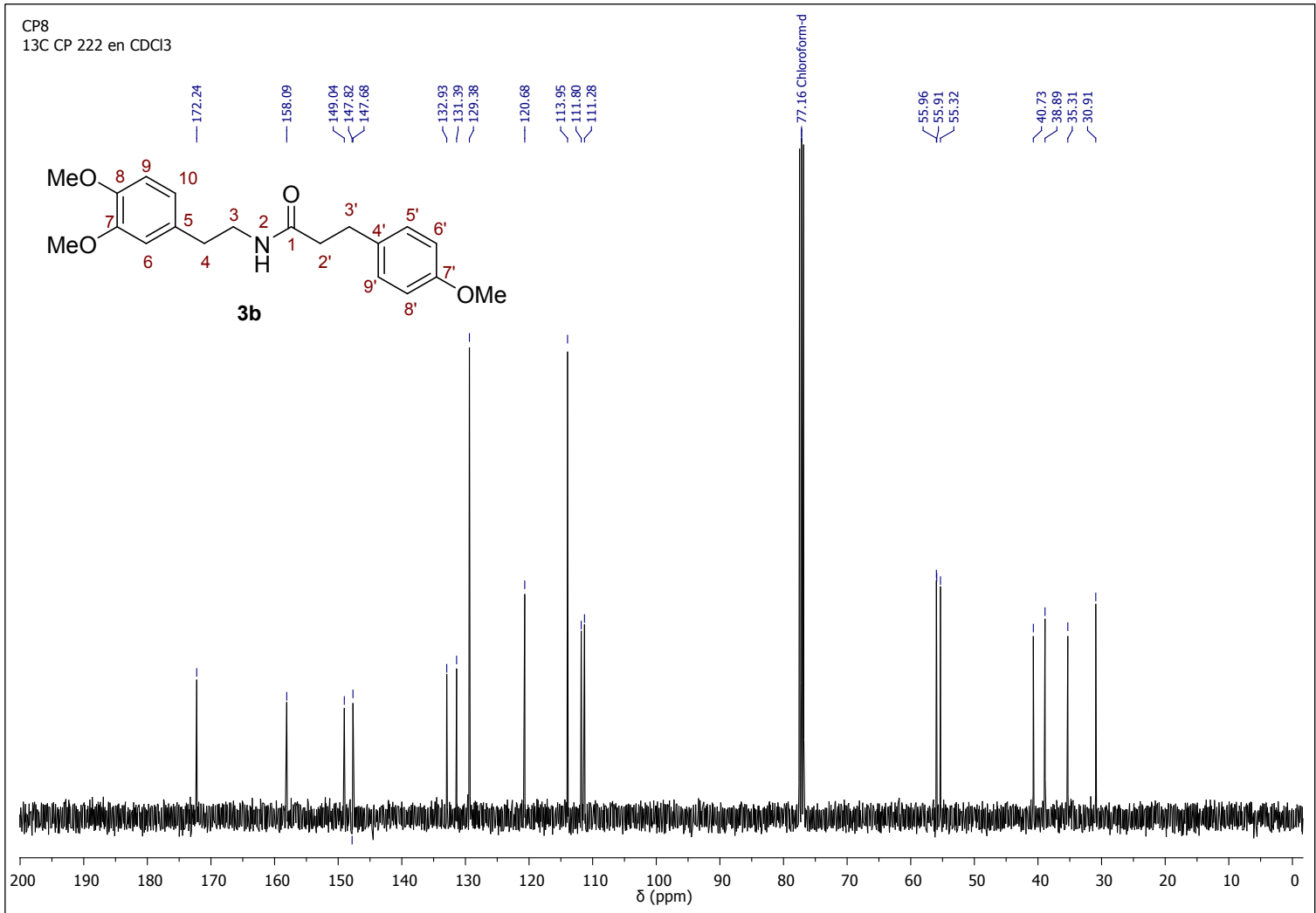

Figure S6. ${ }^{1} \mathrm{H}-\mathrm{NMR}$ spectrum of $N$-(3,4-dimethoxyphenylethyl)-3,4-dimethoxycinnamamide (3c).

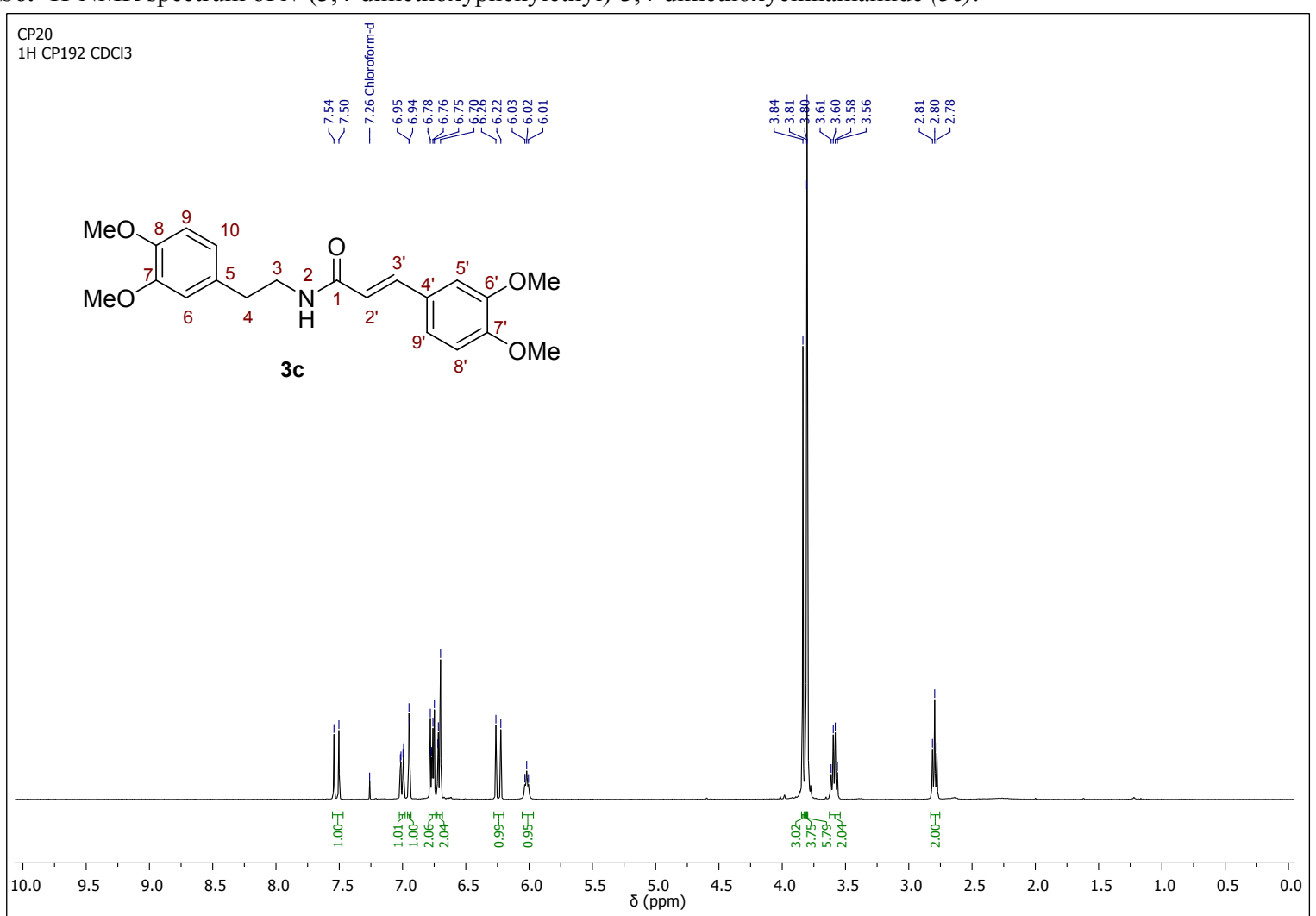


Figure S7. ${ }^{13} \mathrm{C}-\mathrm{NMR}$ spectrum of $N$-(3,4-dimethoxyphenylethyl)-3,4-dimethoxycinnamamide (3c).

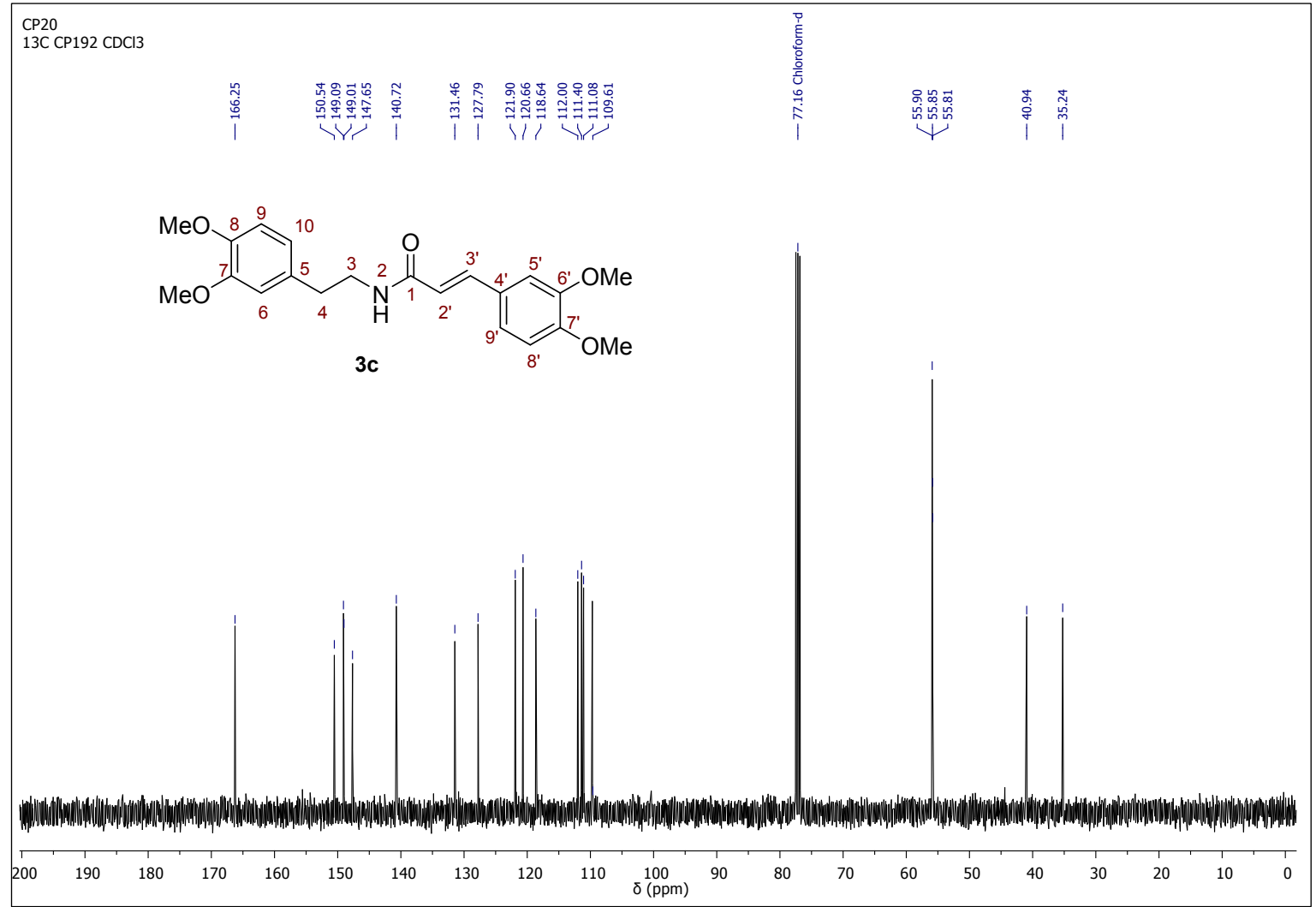

Figure S8. ${ }^{1} \mathrm{H}-\mathrm{NMR}$ spectrum of $N$-(3,4-dimethoxyphenylethyl)-3,4,5-trimethoxycinnamamide (3d).

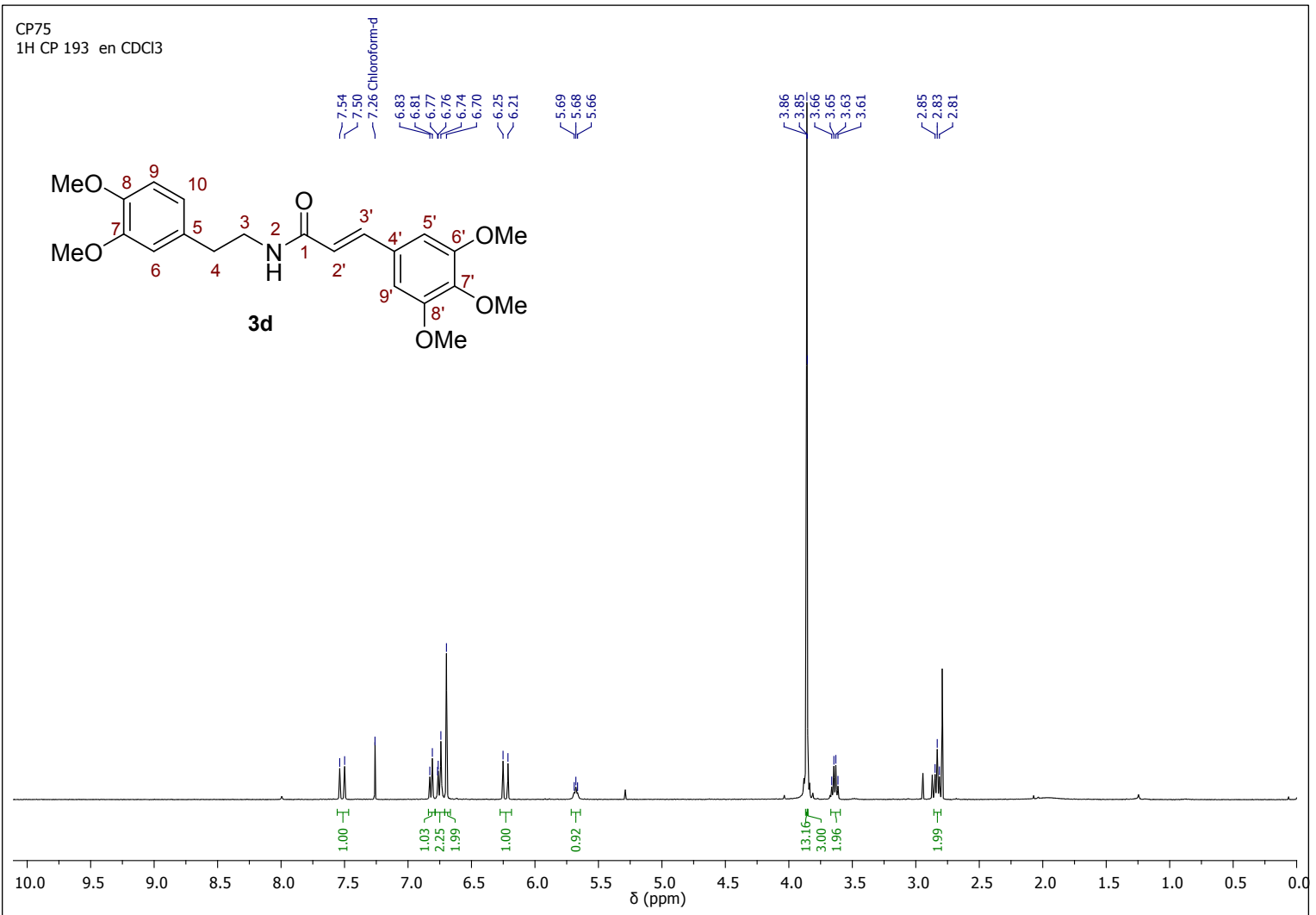


Figure S9. ${ }^{13} \mathrm{C}-\mathrm{NMR}$ spectrum of $N$-(3,4-dimethoxyphenylethyl)-3,4,5-trimethoxycinnamamide (3d).

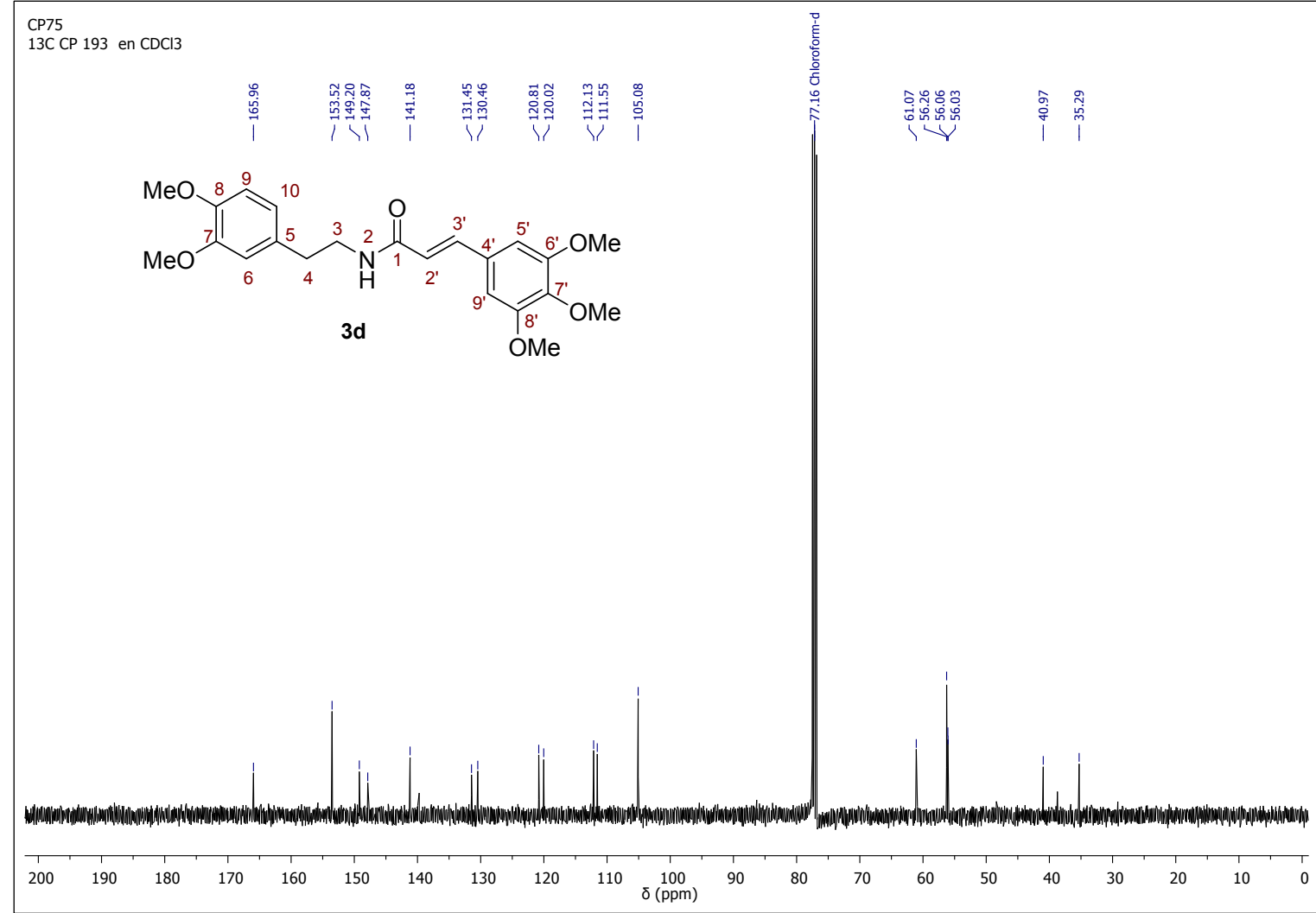

Figure S10. DEPT-135 spectrum of $N$-(3,4-dimethoxyphenylethyl)-3,4,5-trimethoxycinnamamide (3d).

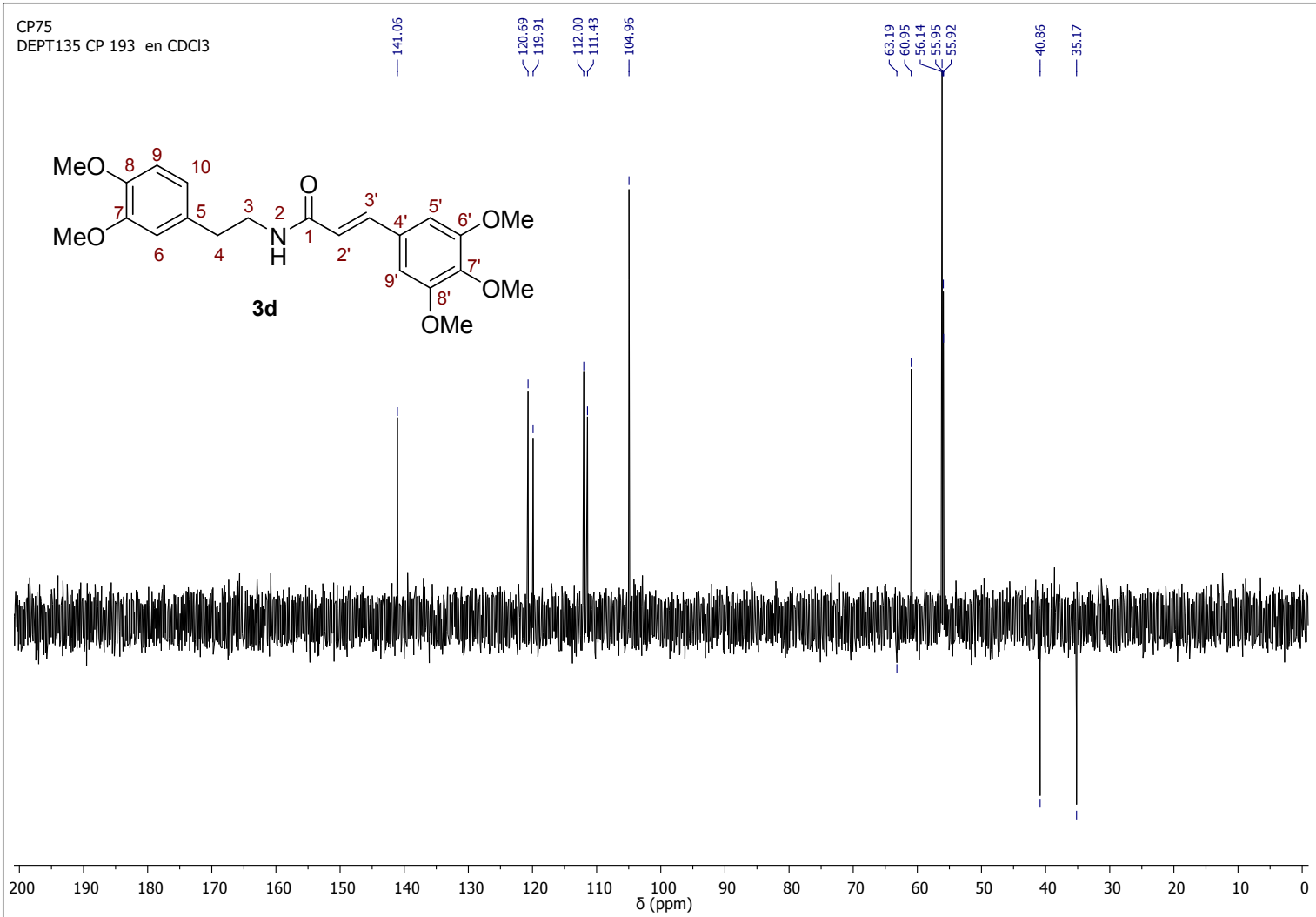


Figure S11. ${ }^{1} \mathrm{H}-\mathrm{NMR}$ spectrum of $N$-(3,4-dimethoxyphenylethyl)-3,4-methylenedioxycinnamamide (3e).

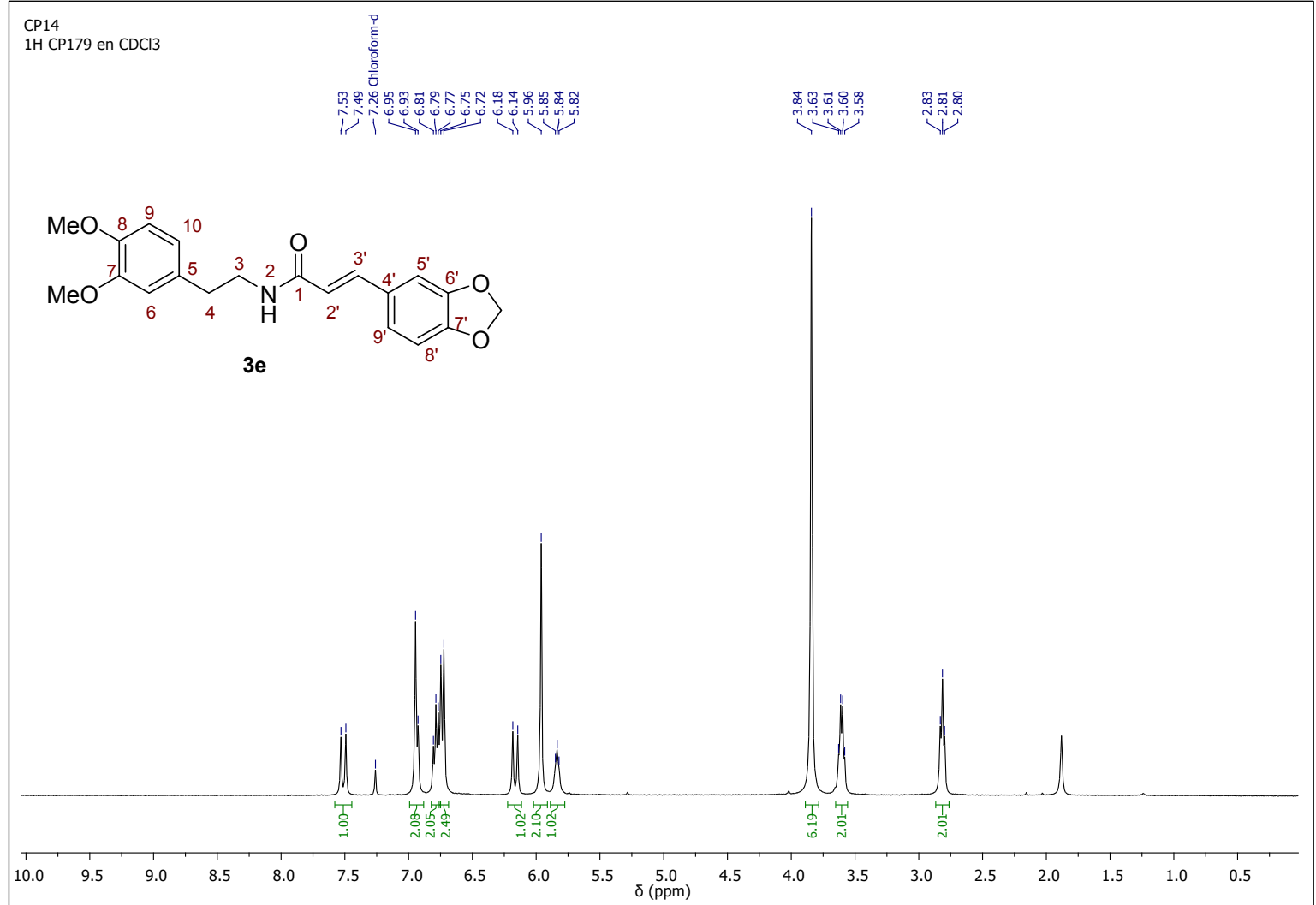

Figure S12. ${ }^{13} \mathrm{C}$-NMR spectrum of $N$-(3,4-dimethoxyphenylethyl)-3,4-methylenedioxycinnamamide (3e).

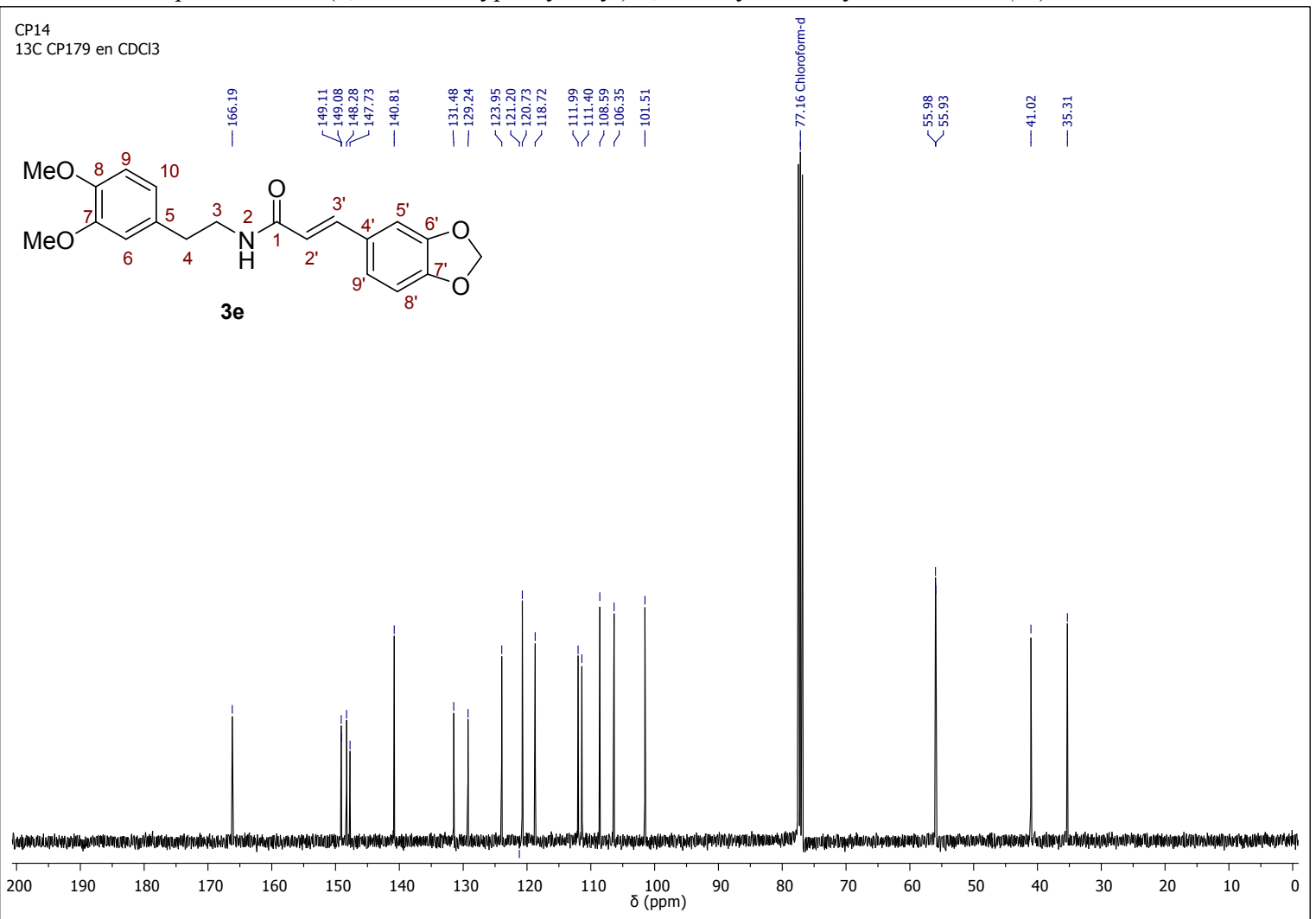


Figure S13. DEPT-135 spectrum of $N$-(3,4-dimethoxyphenylethyl)-3,4-methylenedioxycinnamamide (3e).

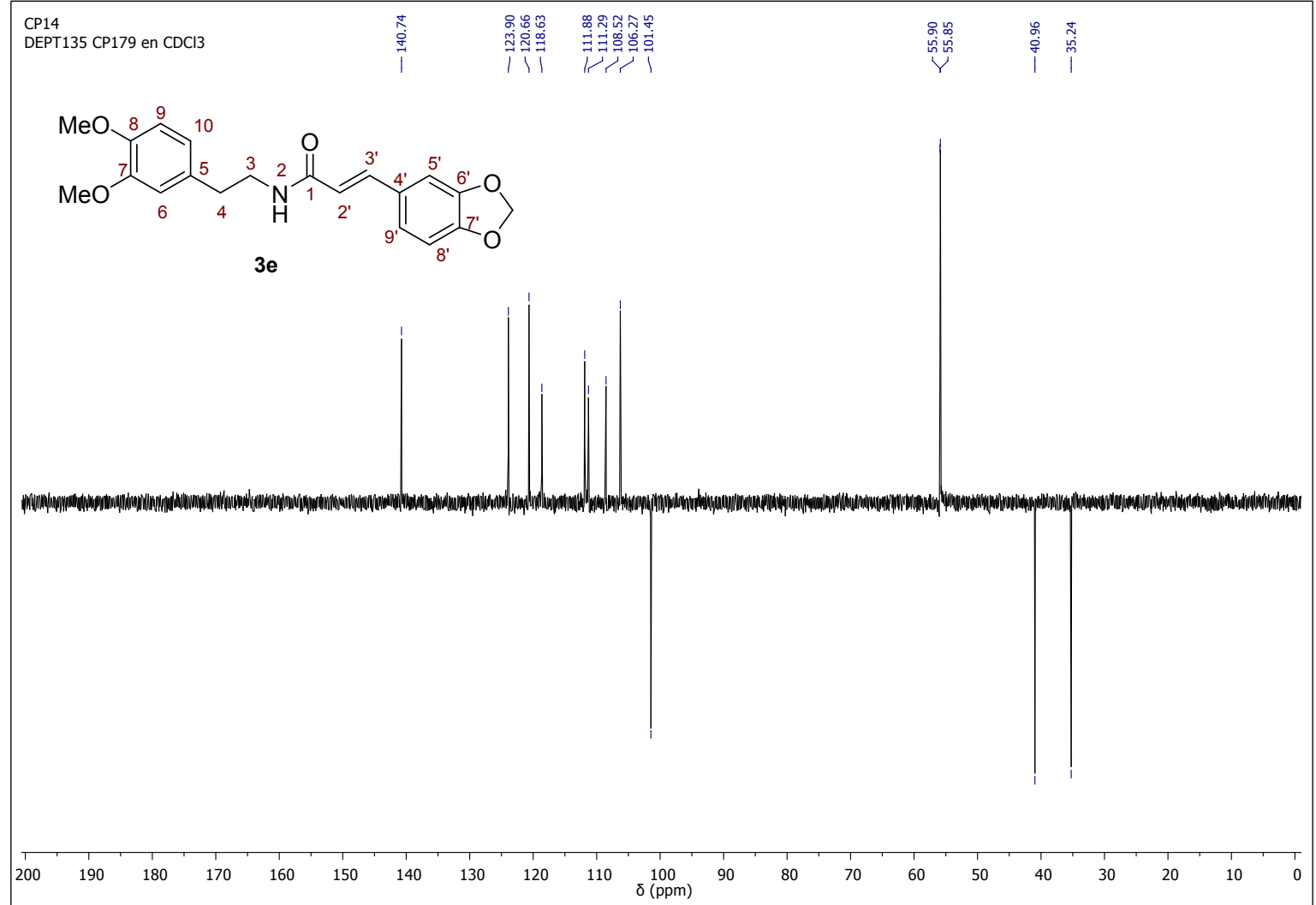

Figure S14. ${ }^{1} \mathrm{H}-\mathrm{NMR}$ spectrum of $N$-(3,4-dimethoxyphenylethyl)-4-acetoxycinnamamide $(3 \boldsymbol{f})$.

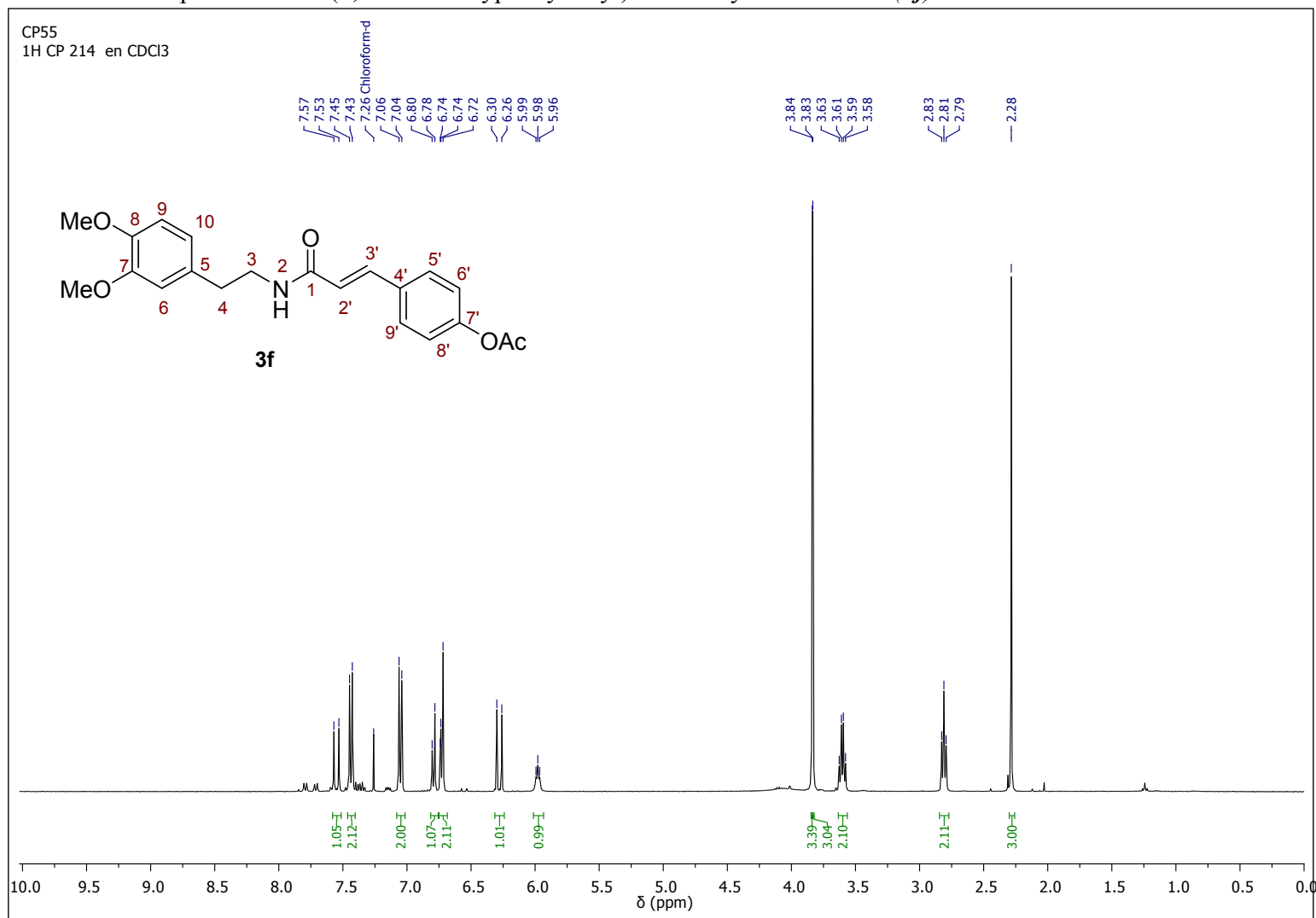


Figure S15. ${ }^{13} \mathrm{C}$-NMR spectrum of $N$-(3,4-dimethoxyphenylethyl)-4-acetoxycinnamamide (3f).

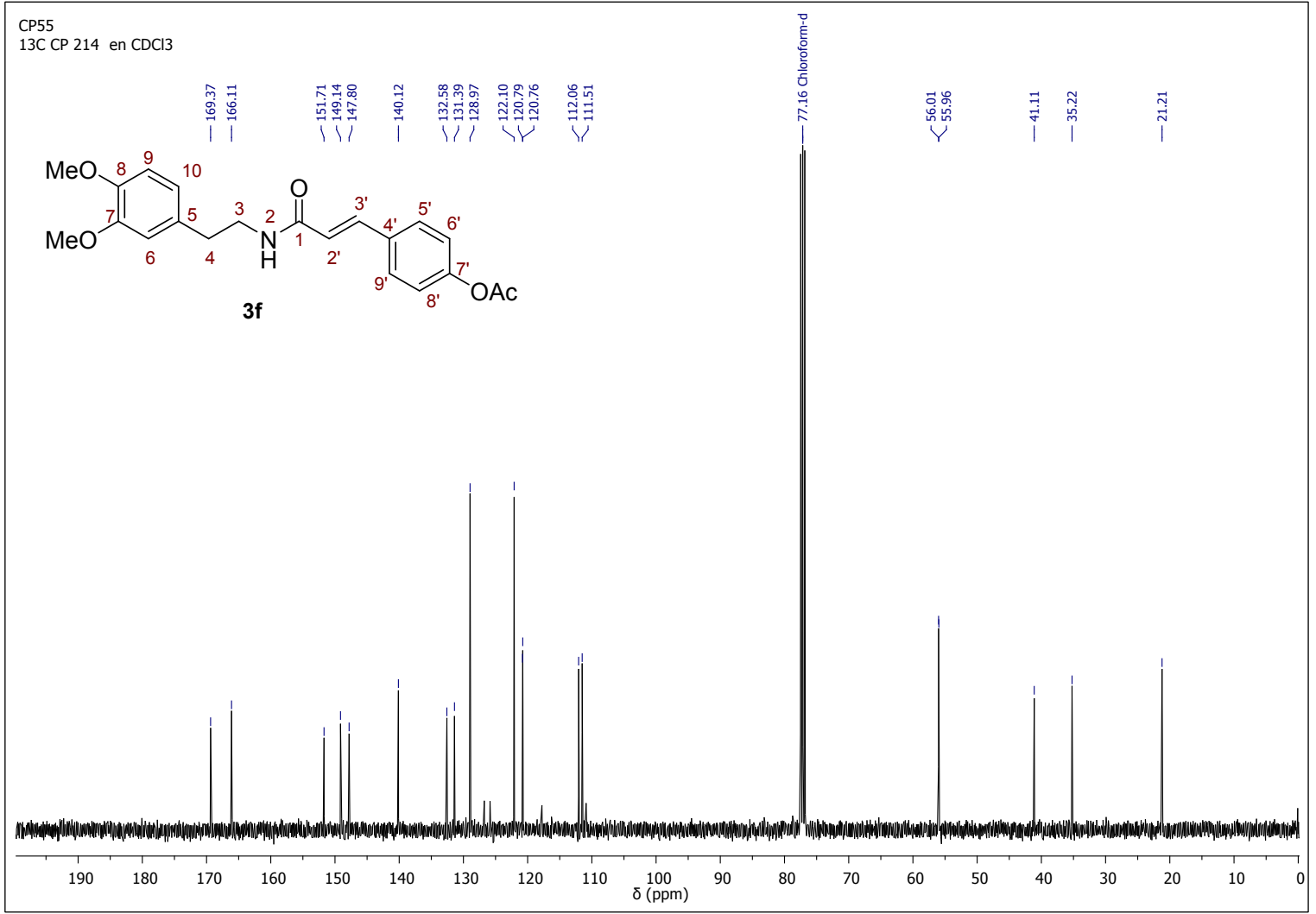

Figure S16. DEPT-135 spectrum of $N$-(3,4-dimethoxyphenylethyl)-4-acetoxycinnamamide (3f).

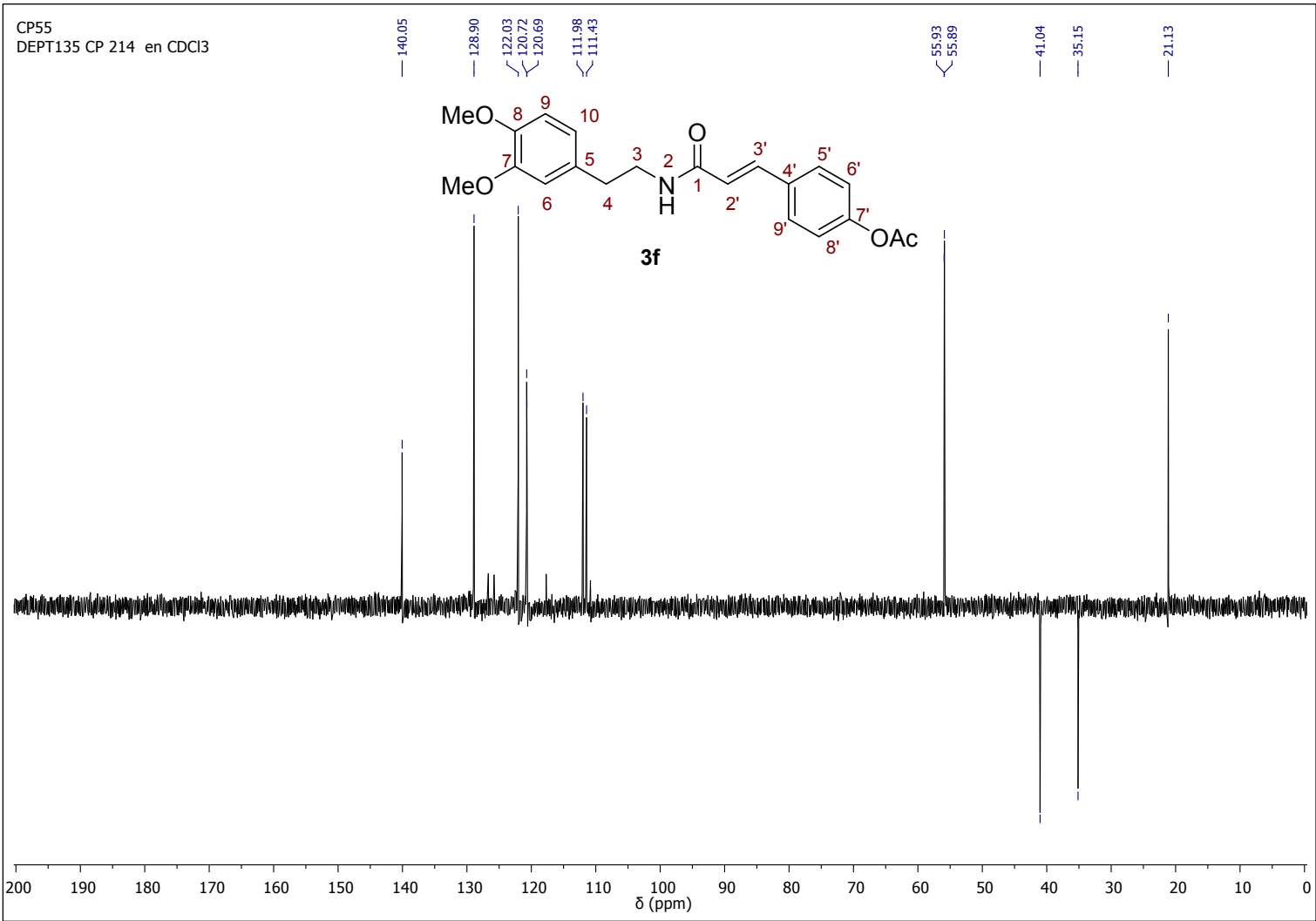


Figure S17. ${ }^{1} \mathrm{H}-\mathrm{NMR}$ spectrum of $N$-(3,4-dimethoxyphenylethyl)-4-acetoxy-3-methoxycinnamamide (3g).

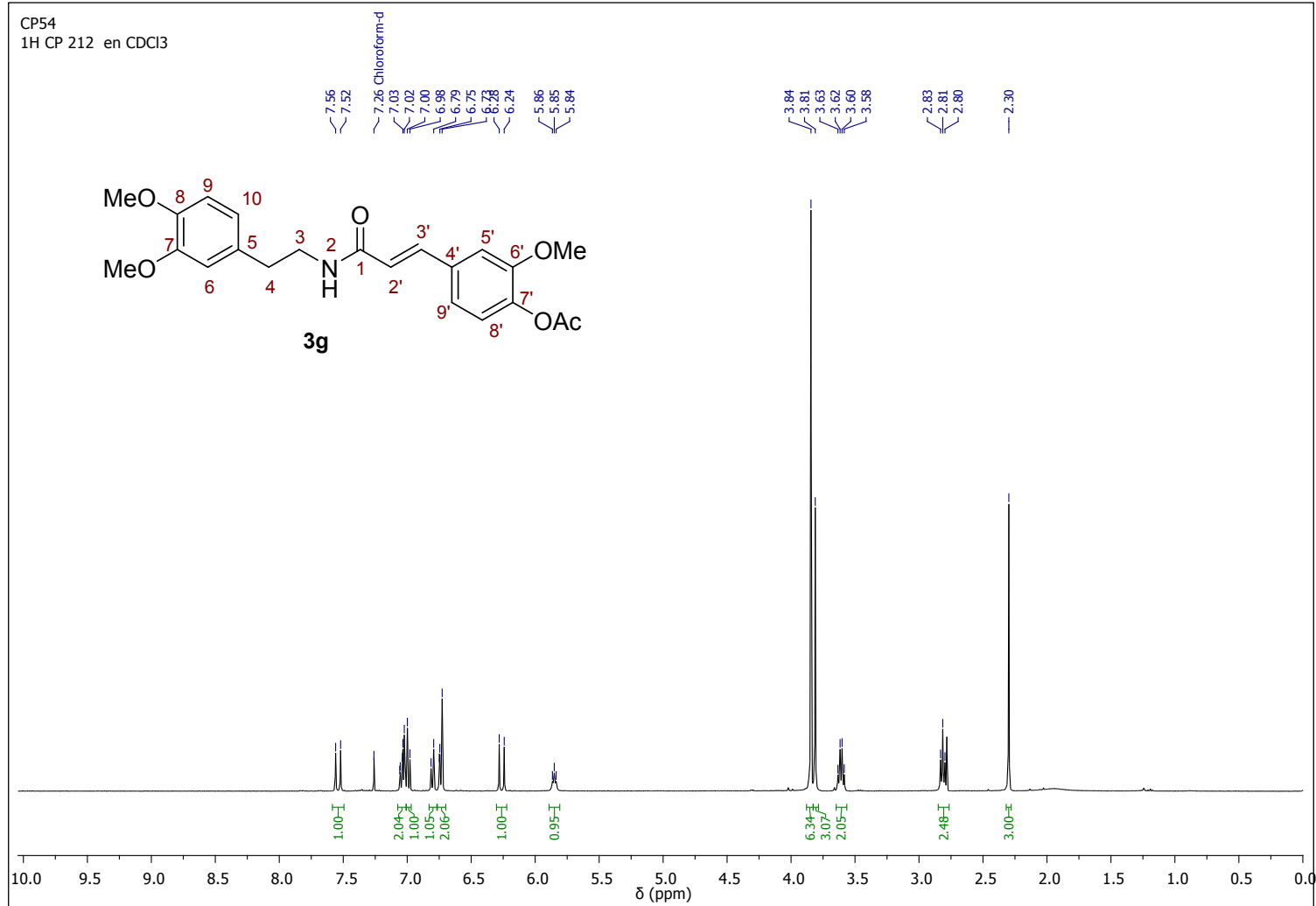

Figure S18. ${ }^{13} \mathrm{C}$-NMR spectrum of $N$-(3,4-dimethoxyphenylethyl)-4-acetoxy-3-methoxycinnamamide (3g).

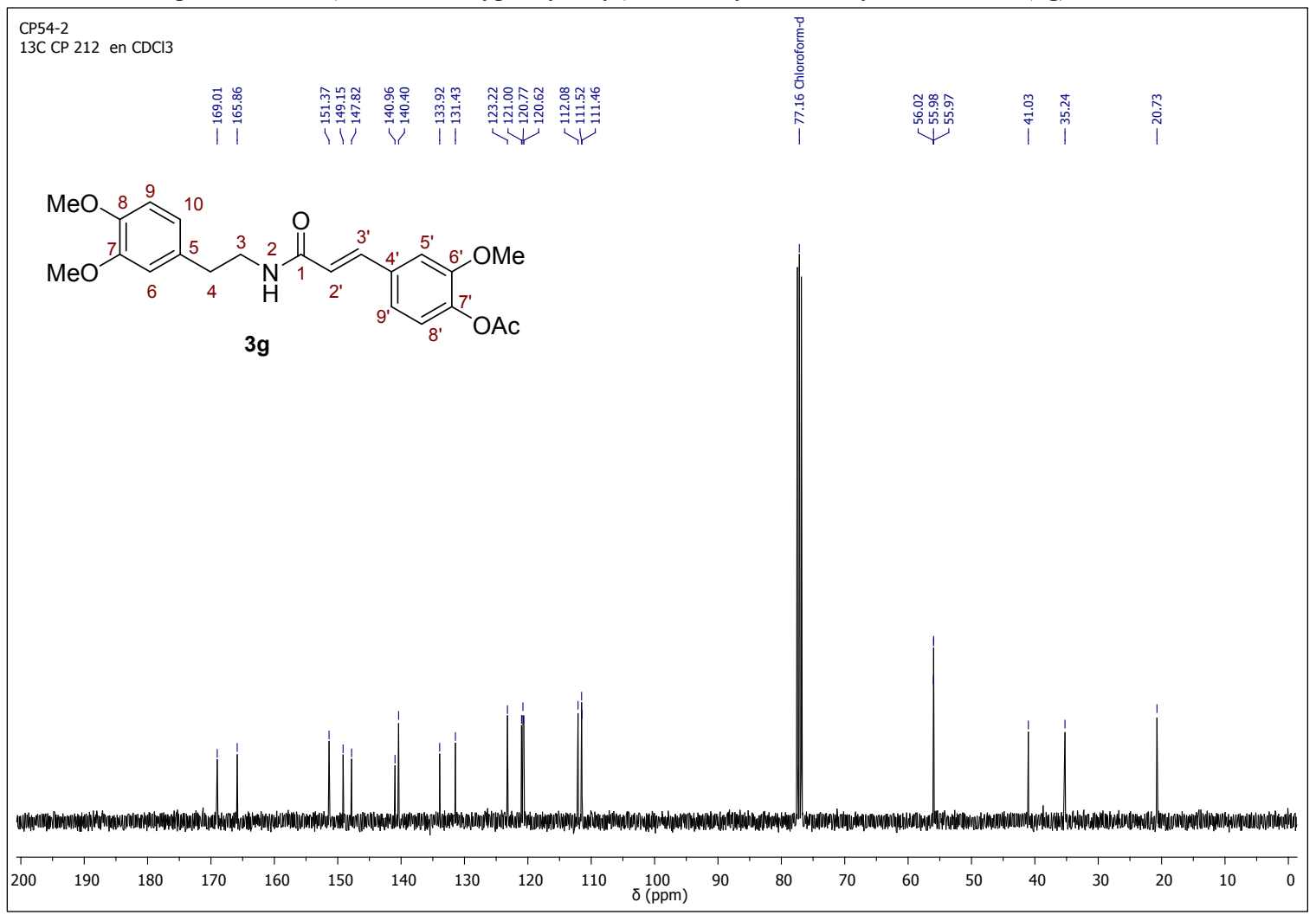


Figure S19. DEPT-135 spectrum of $N$-(3,4-dimethoxyphenylethyl)-4-acetoxy-3-methoxycinnamamide (3g).

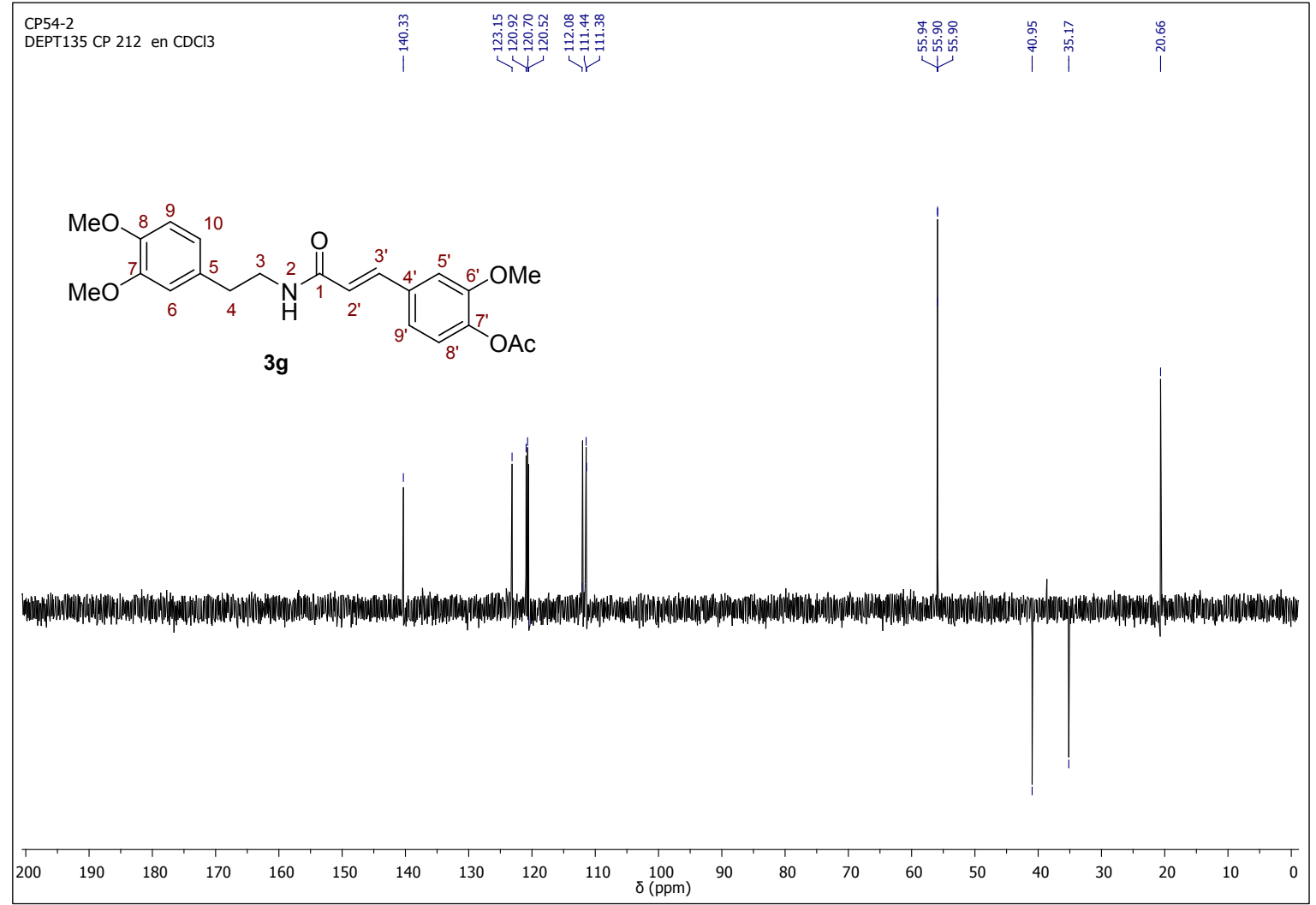

Figure S20. ${ }^{1} \mathrm{H}-\mathrm{NMR}$ spectrum of $N$-(3,4-dimethoxyphenylethyl)-3-acetoxy-4-methoxycinnamamide (3h).

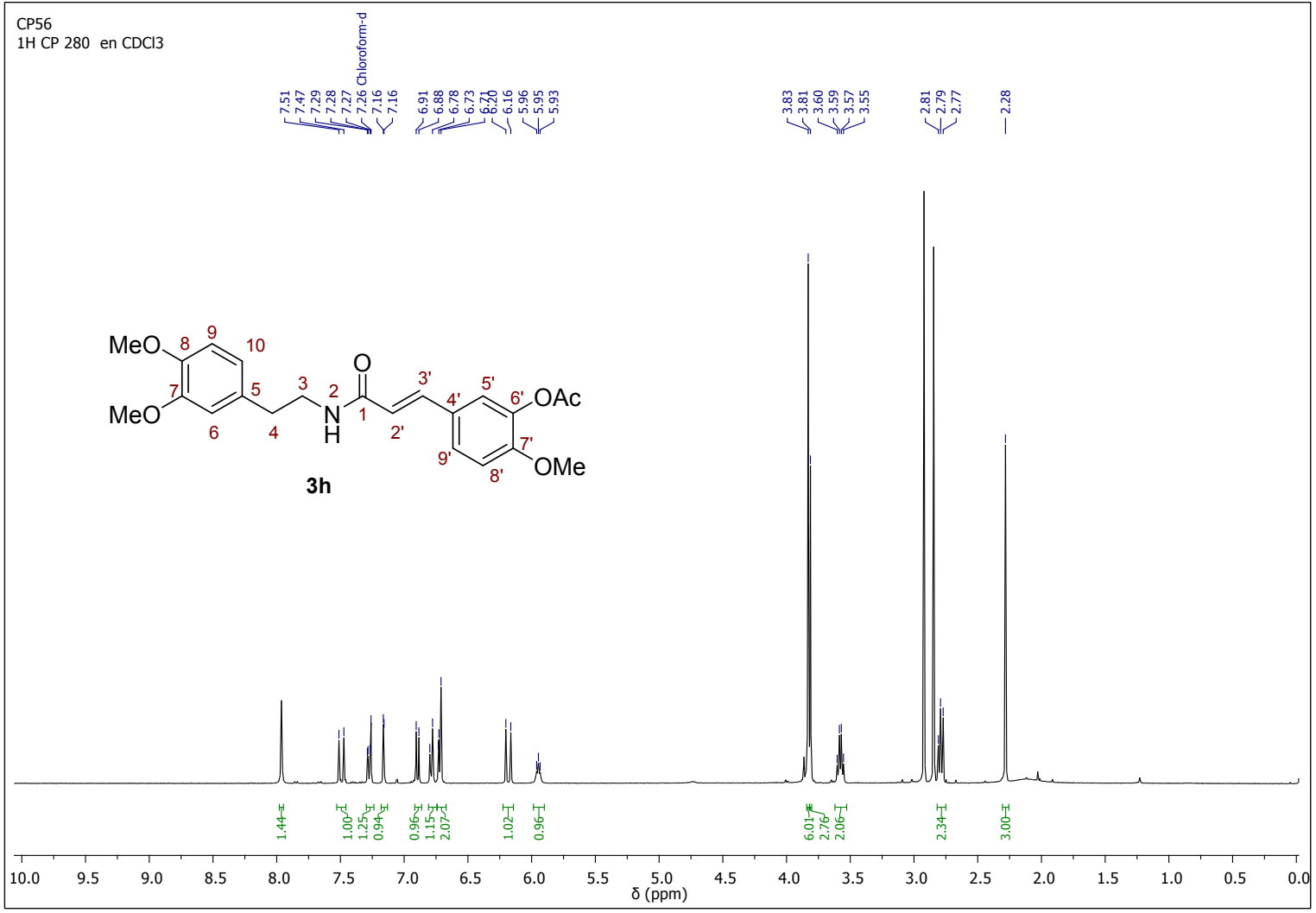


Figure S21. ${ }^{13} \mathrm{C}$-NMR spectrum of $N$-(3,4-dimethoxyphenylethyl)-3-acetoxy-4-methoxycinnamamide (3h).

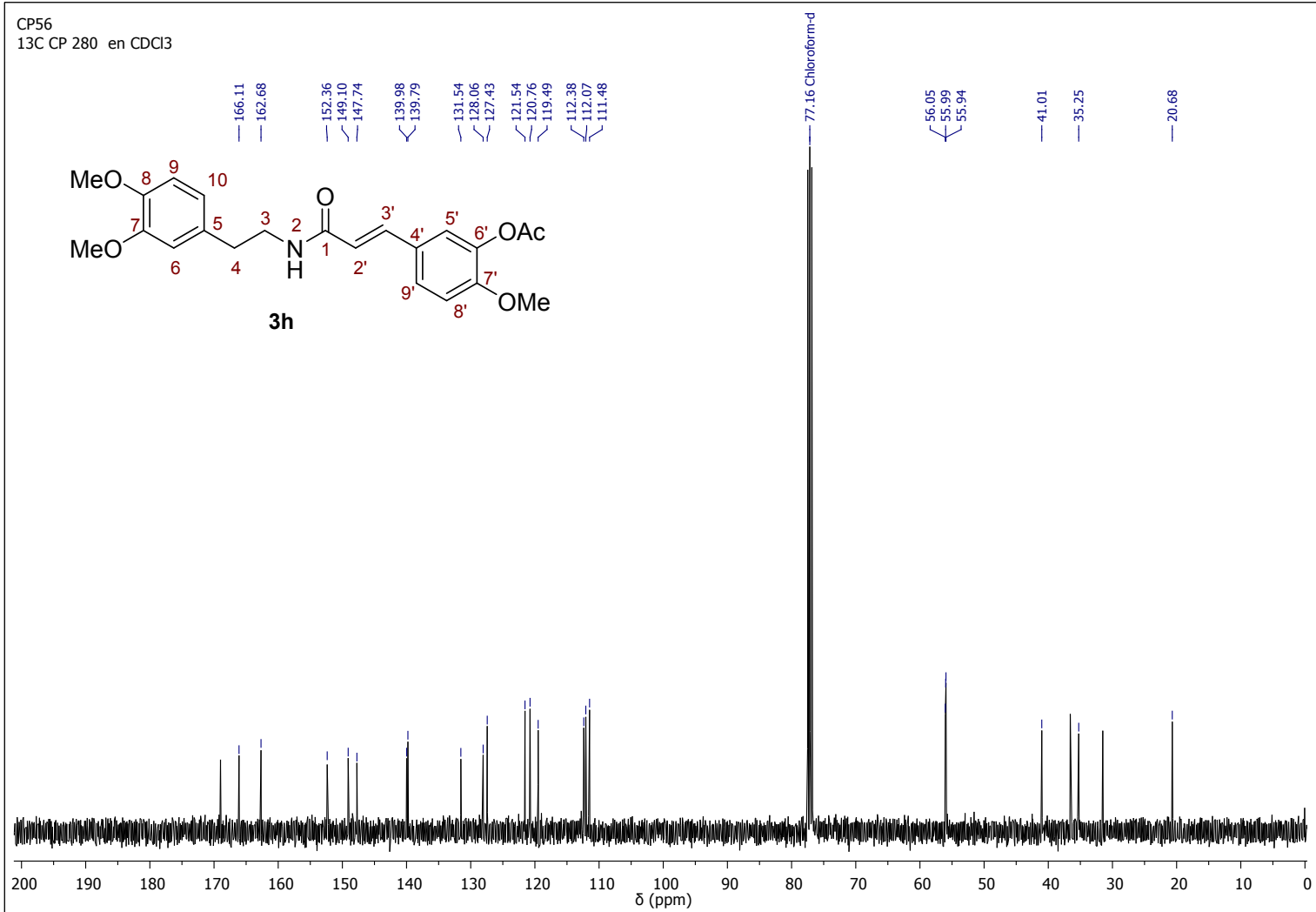

Figure S22. DEPT-135 spectrum of $N$-(3,4-dimethoxyphenylethyl)-3-acetoxy-4-methoxycinnamamide (3h).

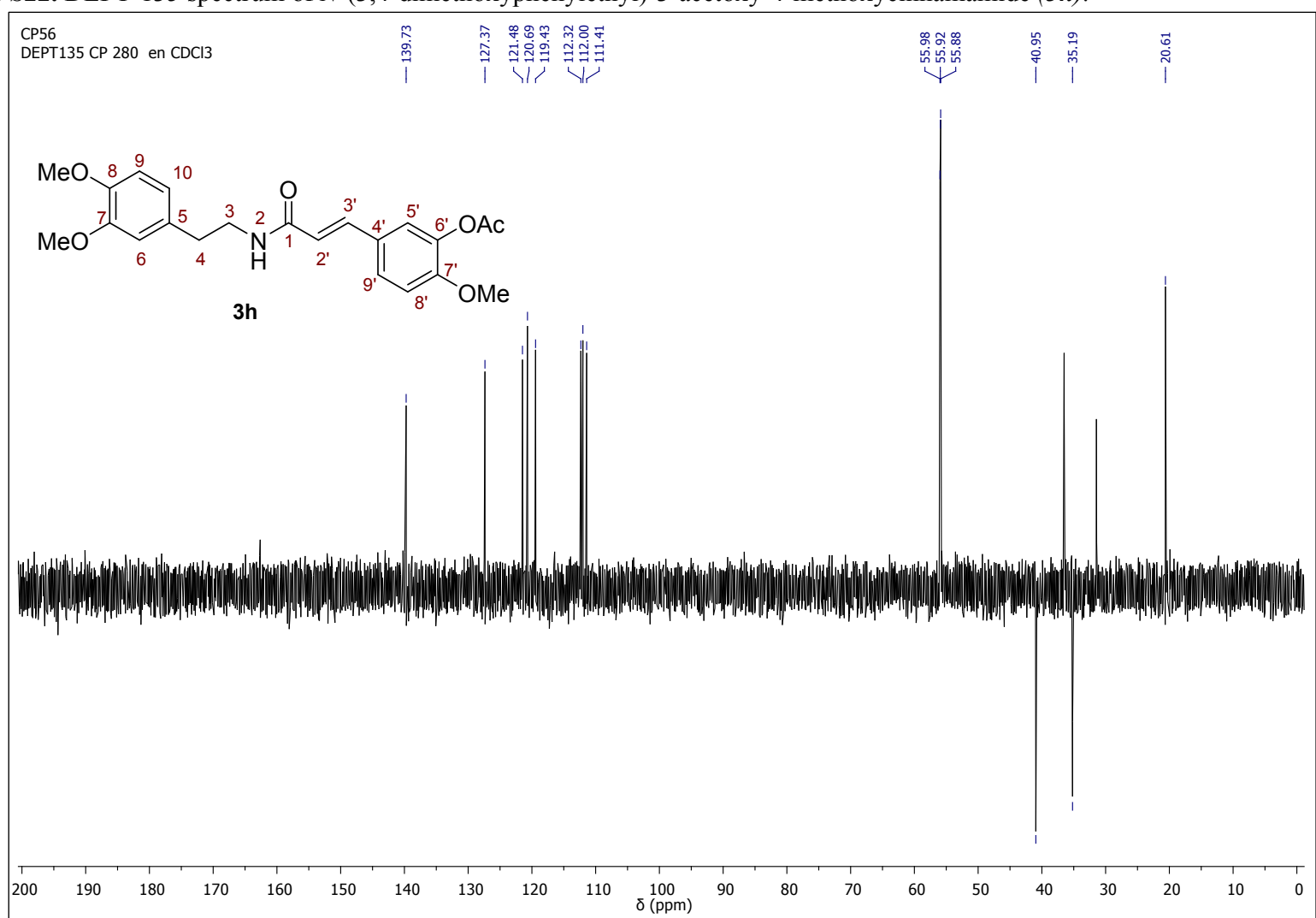


Figure S23. ${ }^{1} \mathrm{H}-\mathrm{NMR}$ spectrum of $N$-(3,4-dimethoxyphenylethyl)-4-acetoxy-3,5-dimethoxycinnamamide (3i).

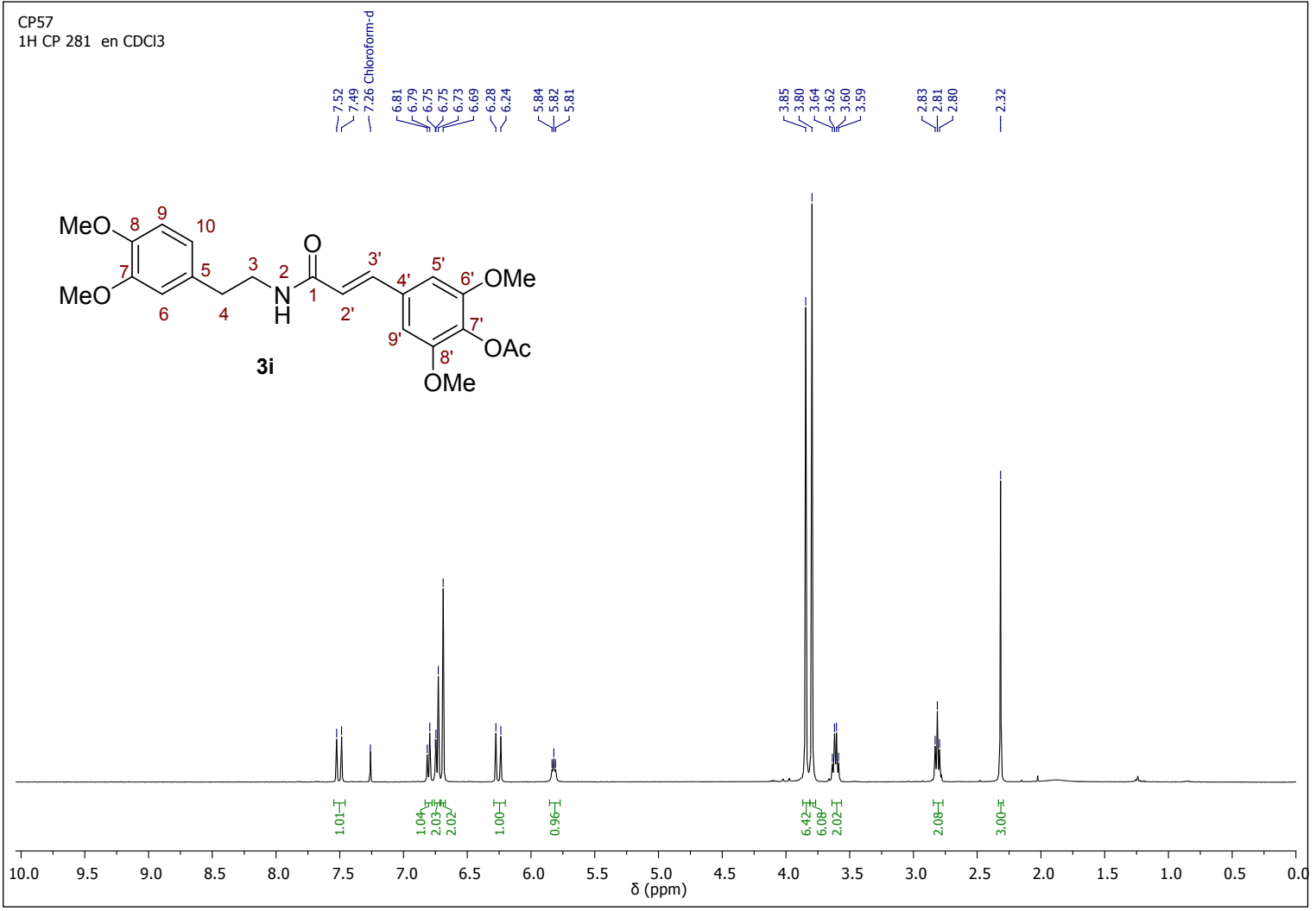

Figure S24. ${ }^{13} \mathrm{C}$-NMR spectrum of $N$-(3,4-dimethoxyphenylethyl)-4-acetoxy-3,5-dimethoxycinnamamide (3i).

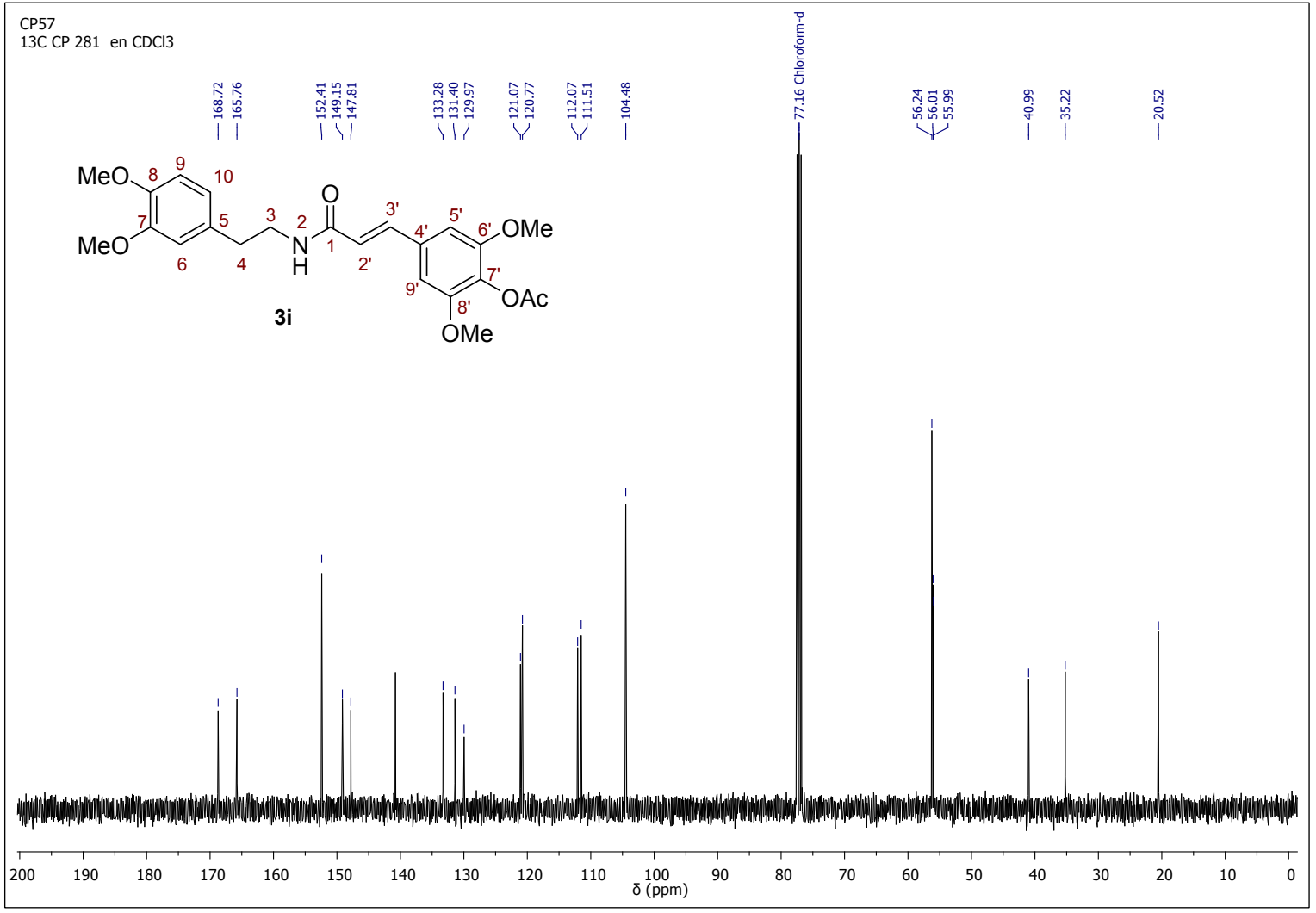


Figure S25. DEPT-135 spectrum of $N$-(3,4-dimethoxyphenylethyl)-4-acetoxy-3,5-dimethoxycinnamamide (3i).

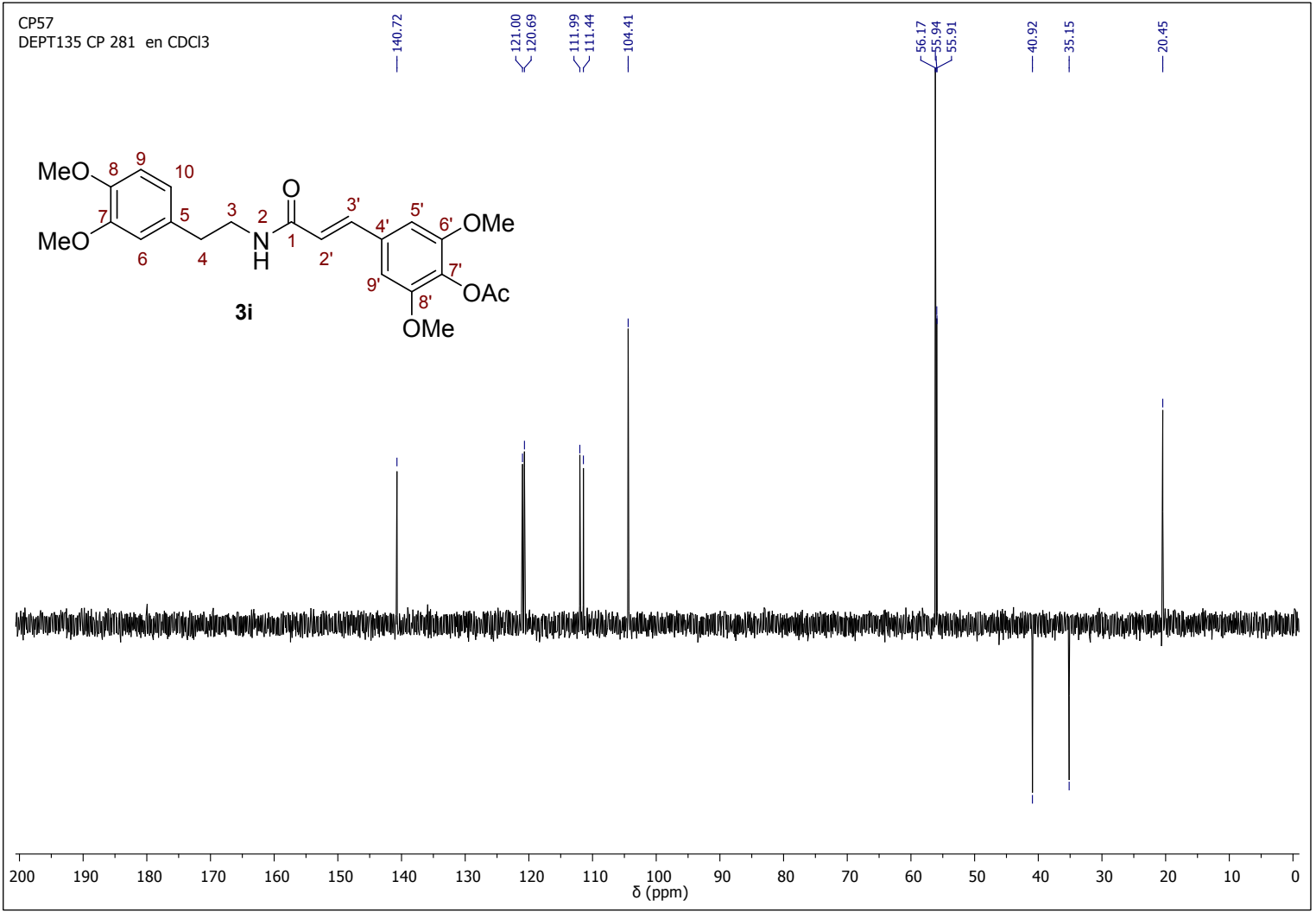




\section{Copies of ${ }^{1} \mathrm{H}$ NMR, ${ }^{13} \mathrm{C}$ NMR and DEPT-135 charts of $N$-phenethyl-3-phenylpropanamides 4a-h}

Figure S26. ${ }^{1} \mathrm{H}-\mathrm{NMR}$ spectrum of $N$-(3,4-dimethoxyphenethyl)-3-phenylpropanamide (4a).

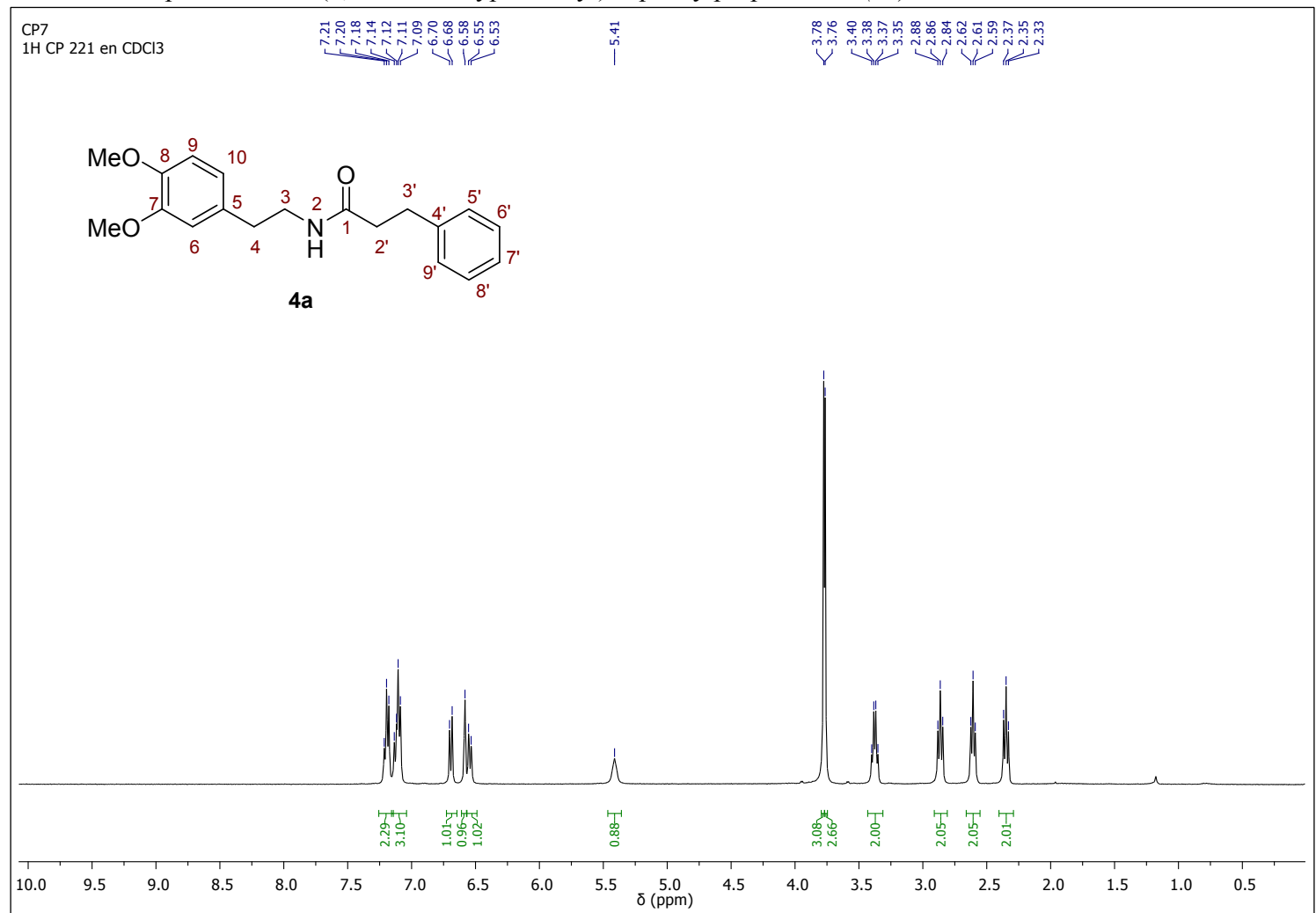

Figure S27. ${ }^{13} \mathrm{C}-\mathrm{NMR}$ spectrum of $N$-(3,4-dimethoxyphenethyl)-3-phenylpropanamide (4a).

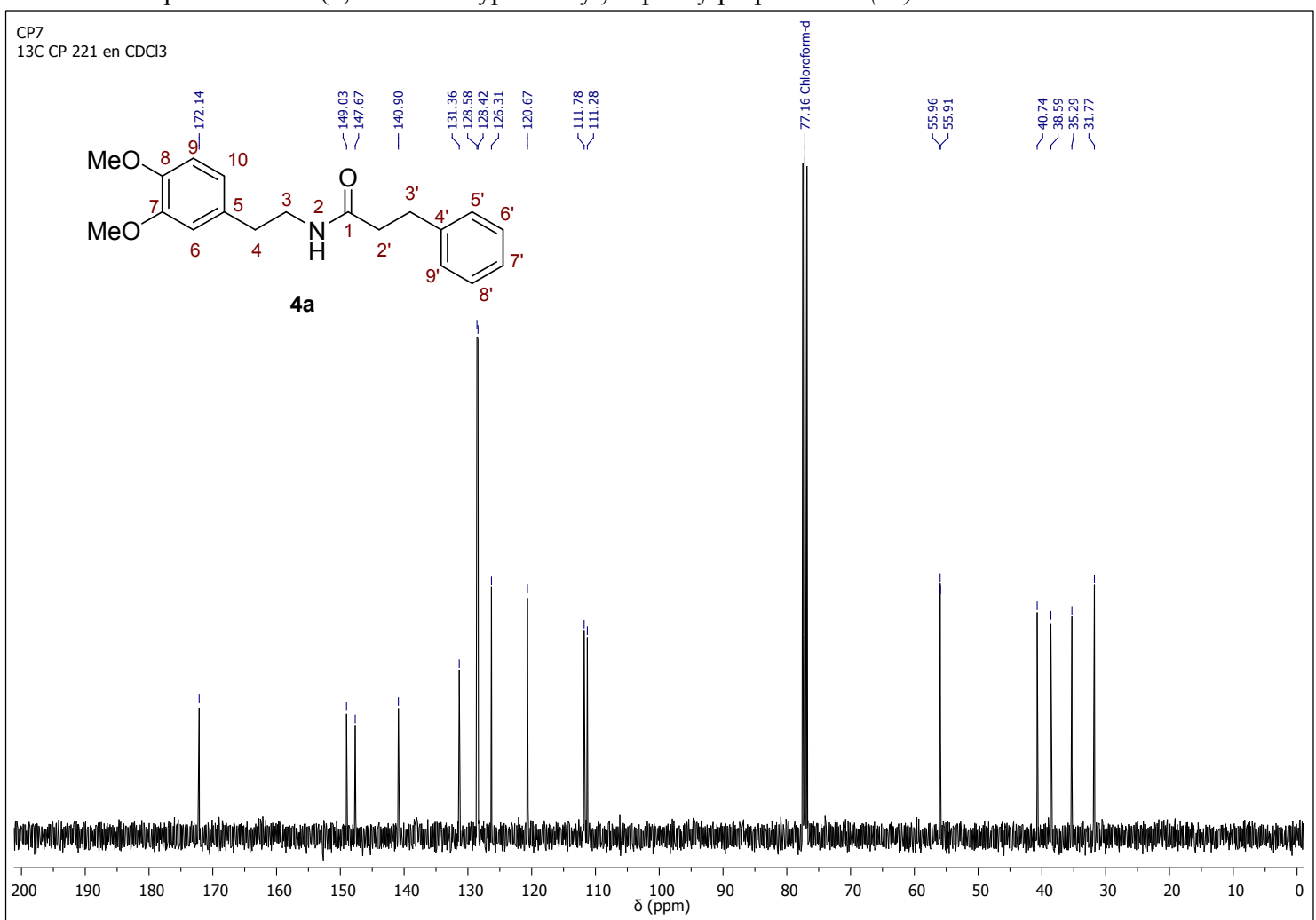


Figure S28. ${ }^{1} \mathrm{H}-\mathrm{NMR}$ spectrum of $N$-(3,4-dimethoxyphenethyl)-3-(3,4-dimethoxyphenyl)propanamide (4b).

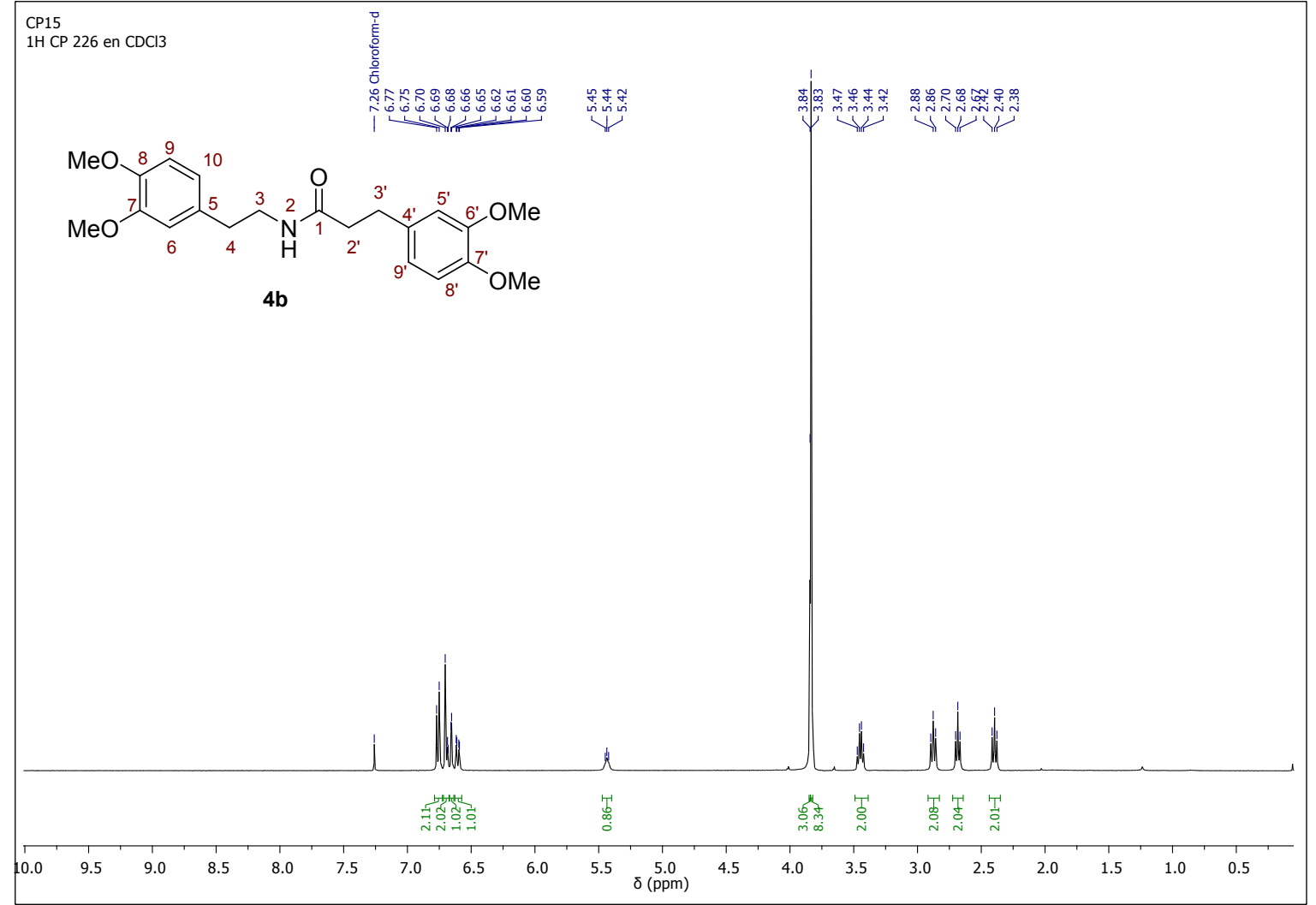

Figure S29. ${ }^{13} \mathrm{C}$-NMR spectrum of $N$-(3,4-dimethoxyphenethyl)-3-(3,4-dimethoxyphenyl)propanamide (4b).

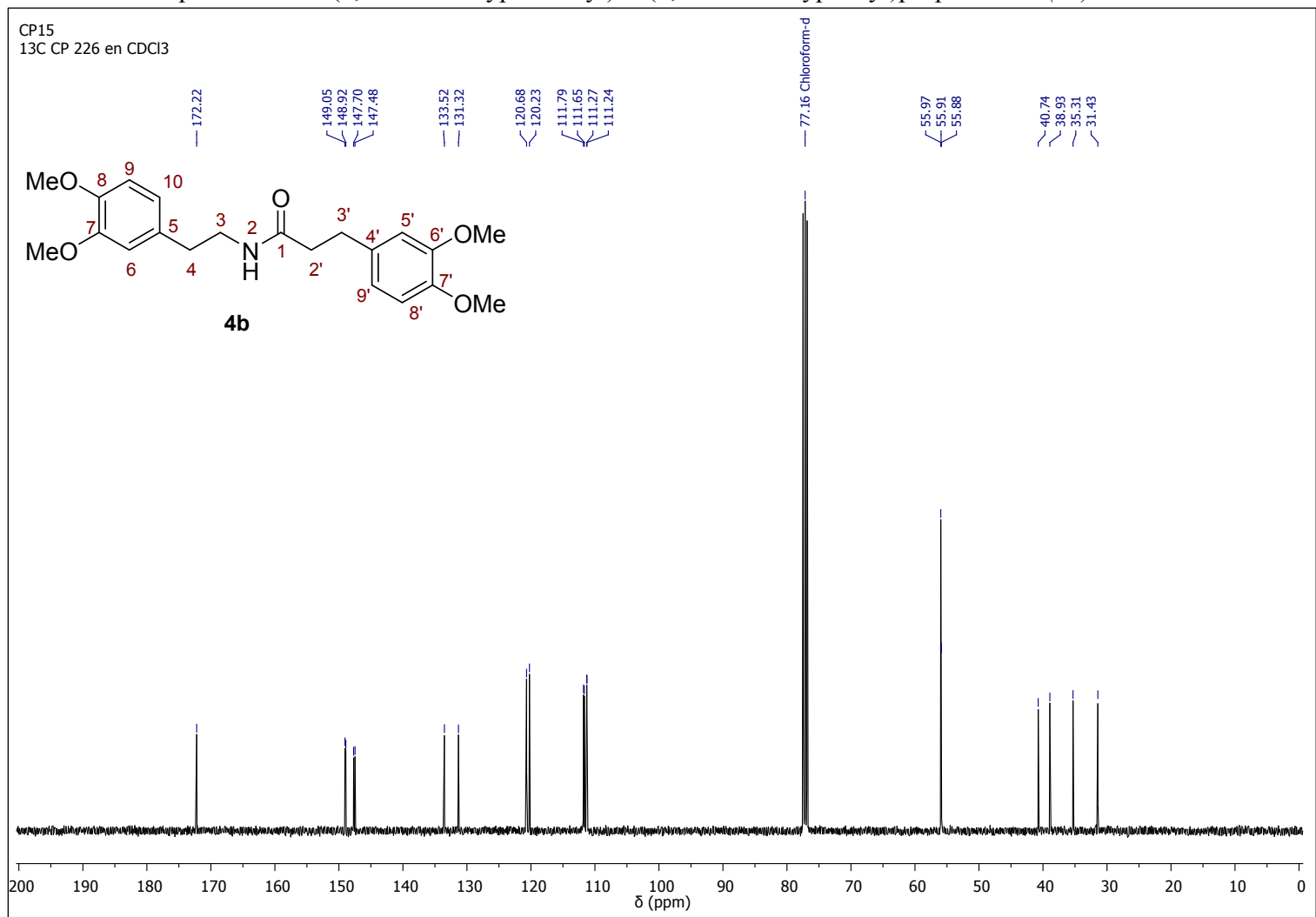


Figure S30. ${ }^{1} \mathrm{H}-\mathrm{NMR}$ spectrum of $N$-(3,4-dimethoxyphenethyl)-3-(3,4,5-trimethoxyphenyl)propanamide (4c).

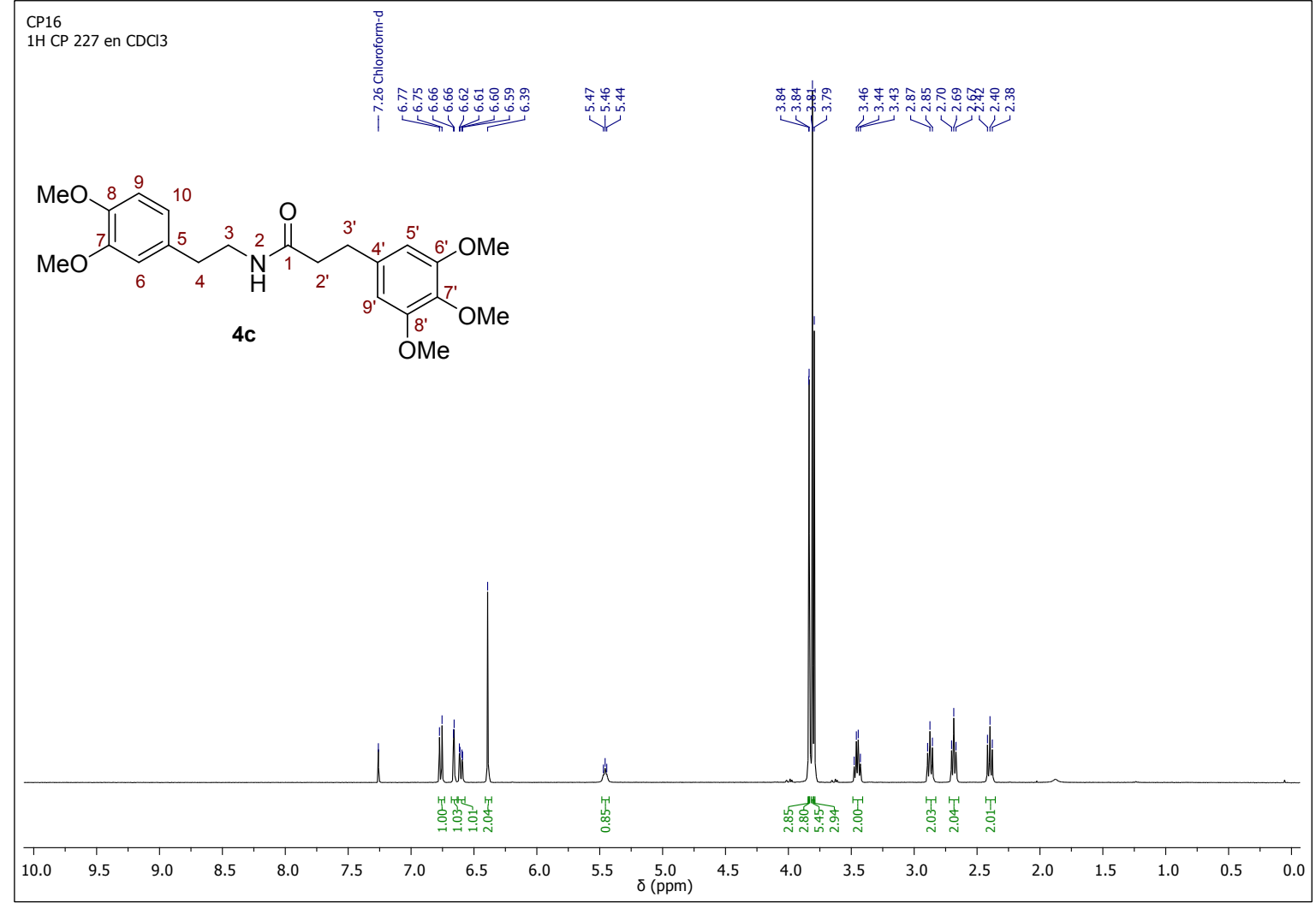

Figure S31. ${ }^{13} \mathrm{C}$-NMR spectrum of $N$-(3,4-dimethoxyphenethyl)-3-(3,4,5-trimethoxyphenyl)propanamide (4c).

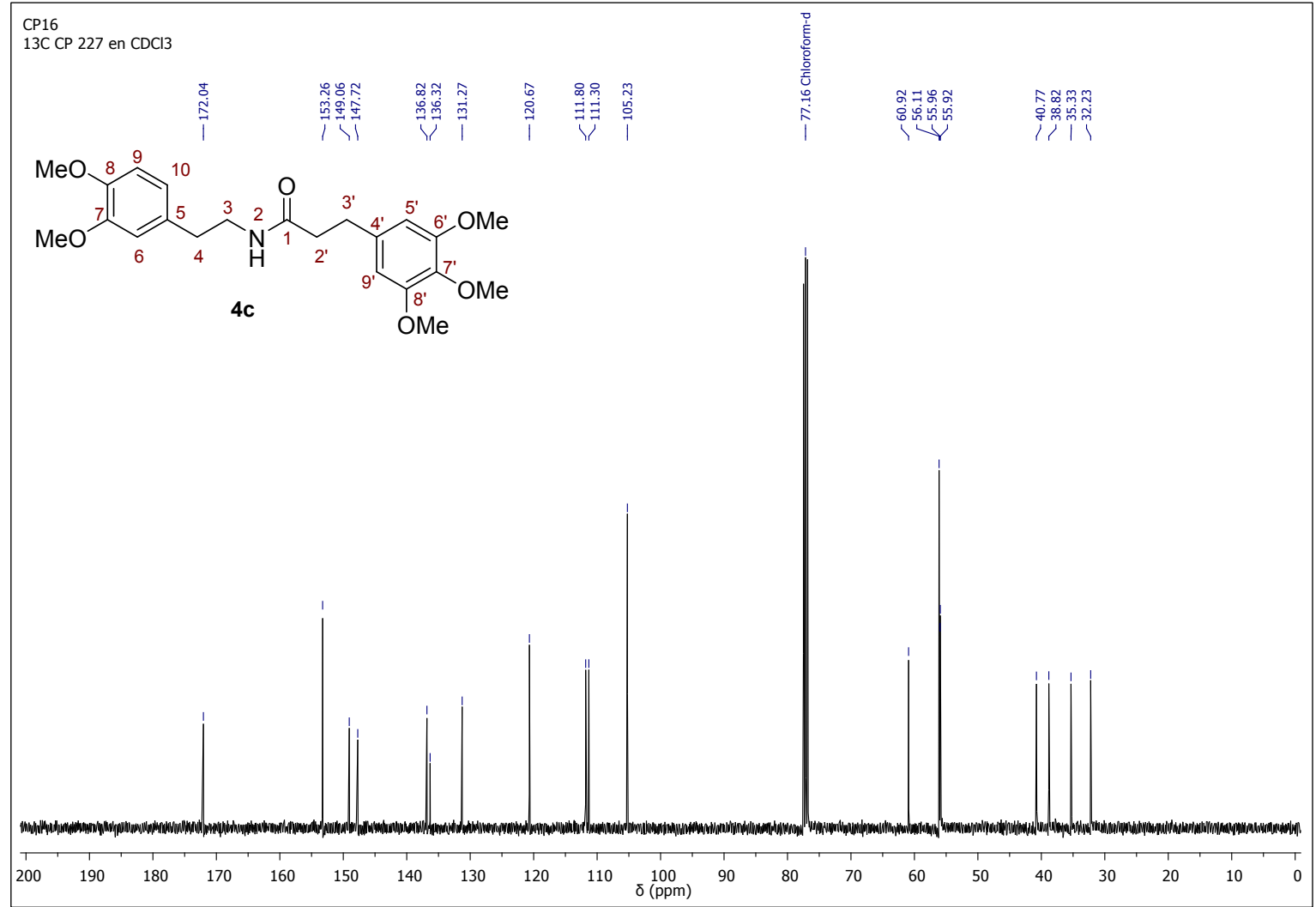


Figure S32. ${ }^{1} \mathrm{H}-\mathrm{NMR}$ spectrum of $N$-(3,4-dimethoxyphenethyl)-3-(3,4-methylendioxyphenyl)propanamide (4d).

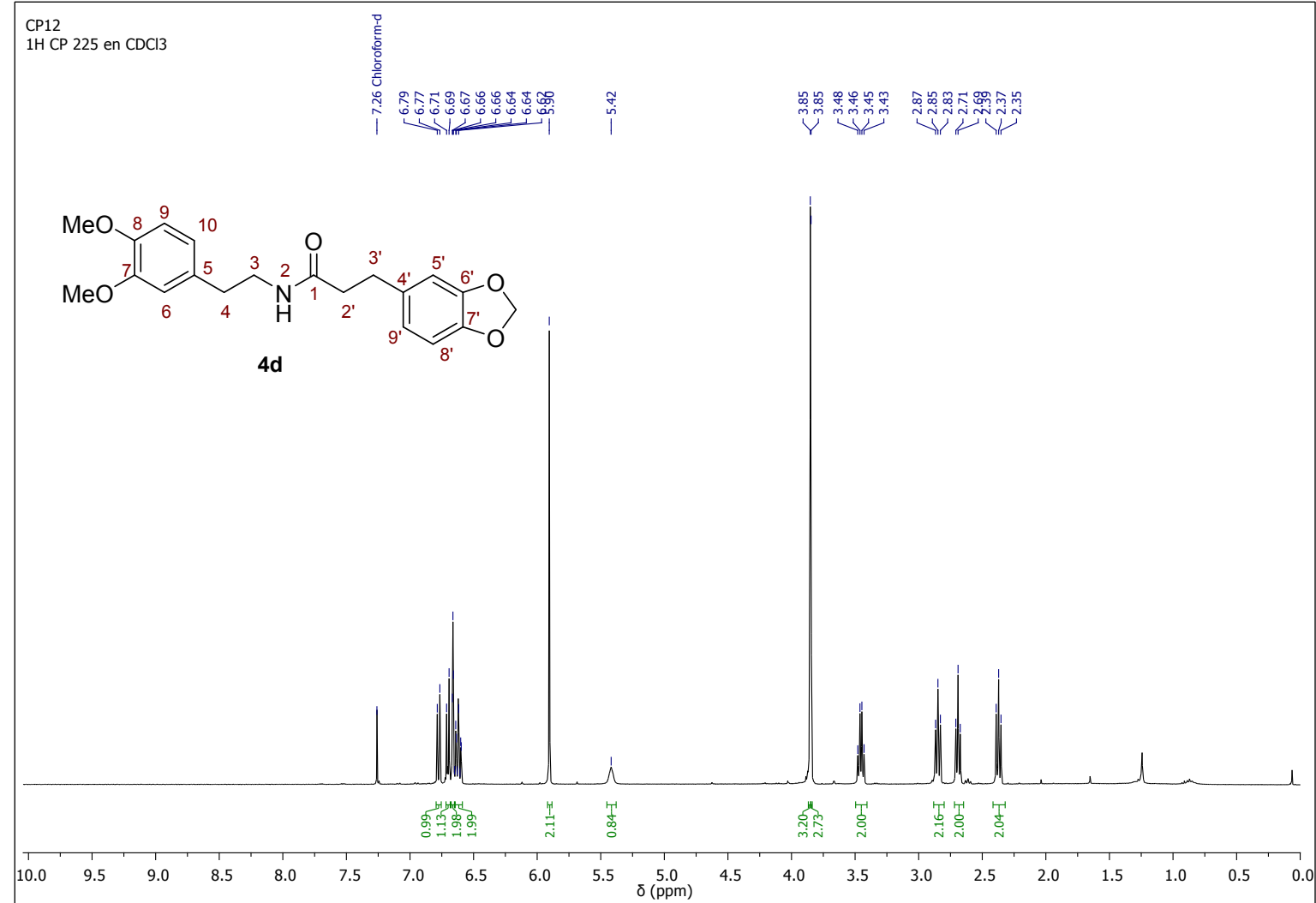

Figure S33. ${ }^{13} \mathrm{C}$-NMR spectrum of $N$-(3,4-dimethoxyphenethyl)-3-(3,4-methylendioxyphenyl)propanamide (4d).

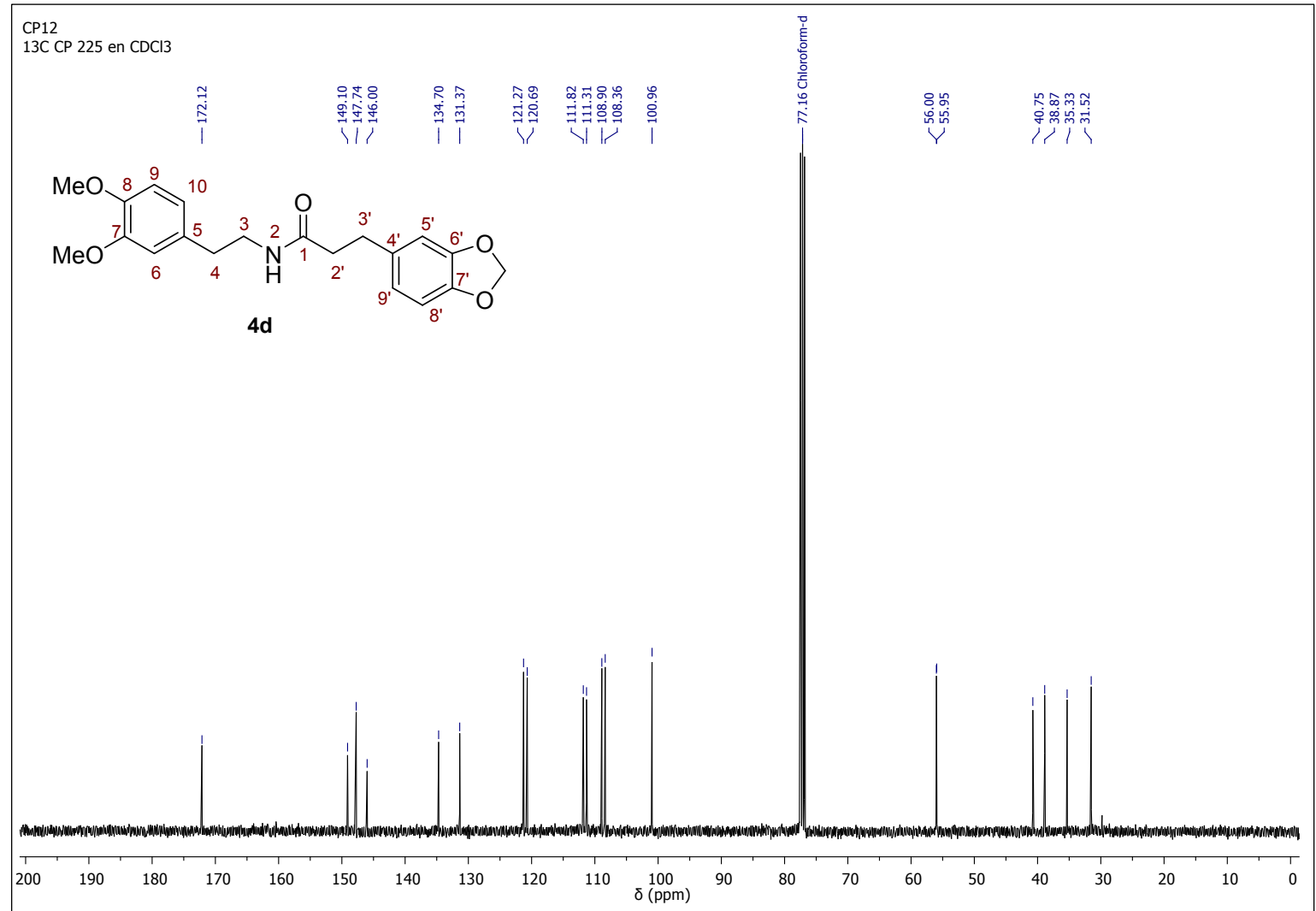


Figure S34. ${ }^{1} \mathrm{H}-\mathrm{NMR}$ spectrum of $N$-(3,4-dimethoxyphenethyl)-3-(4-acetoxyphenyl)propanamide (4e).

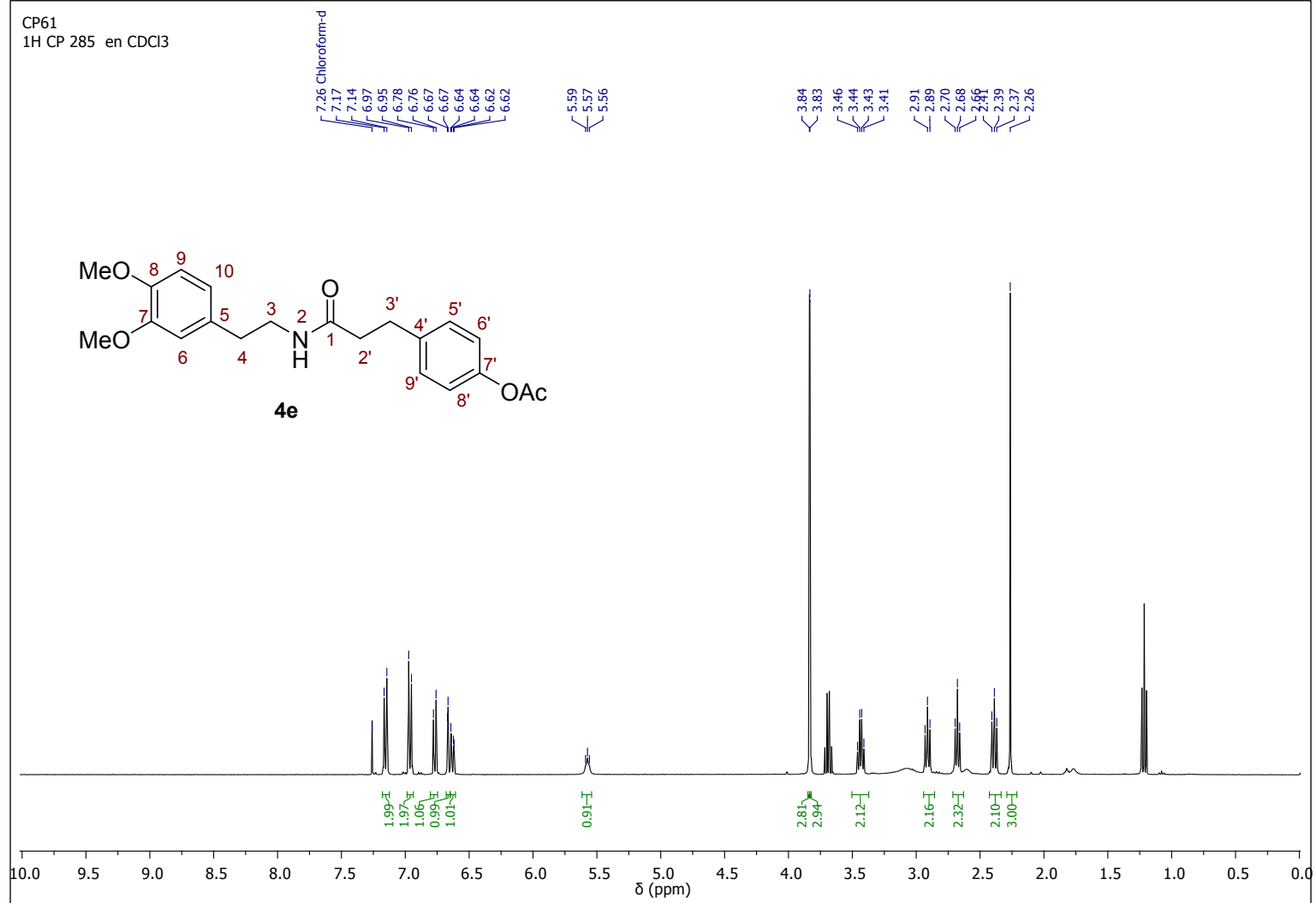

Figure S35. ${ }^{13} \mathrm{C}$-NMR spectrum of $N$-(3,4-dimethoxyphenethyl)-3-(4-acetoxyphenyl)propanamide (4e).

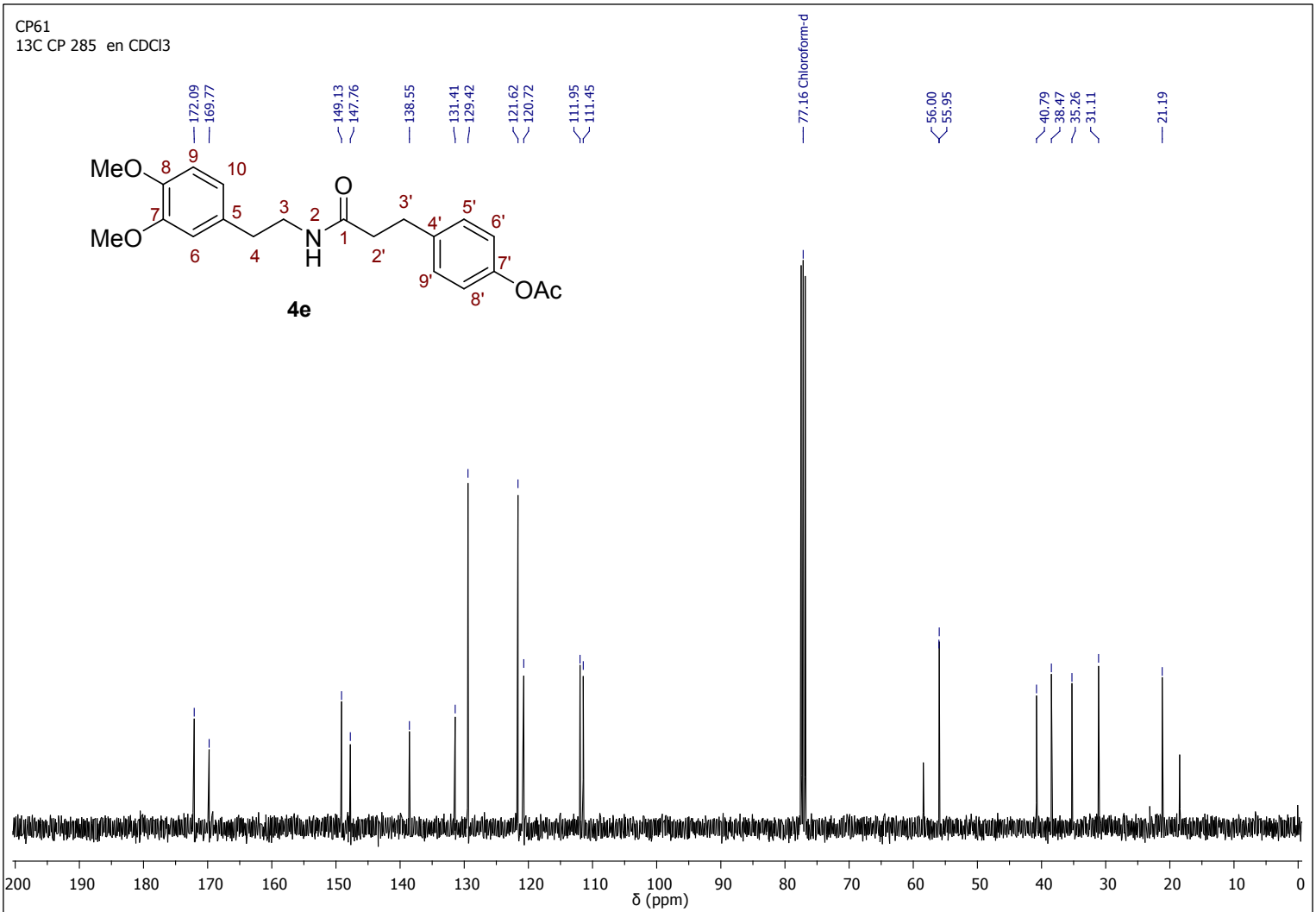


Figure S36. DEPT-135 spectrum of $N$-(3,4-dimethoxyphenethyl)-3-(4-acetoxyphenyl)propanamide (4e).

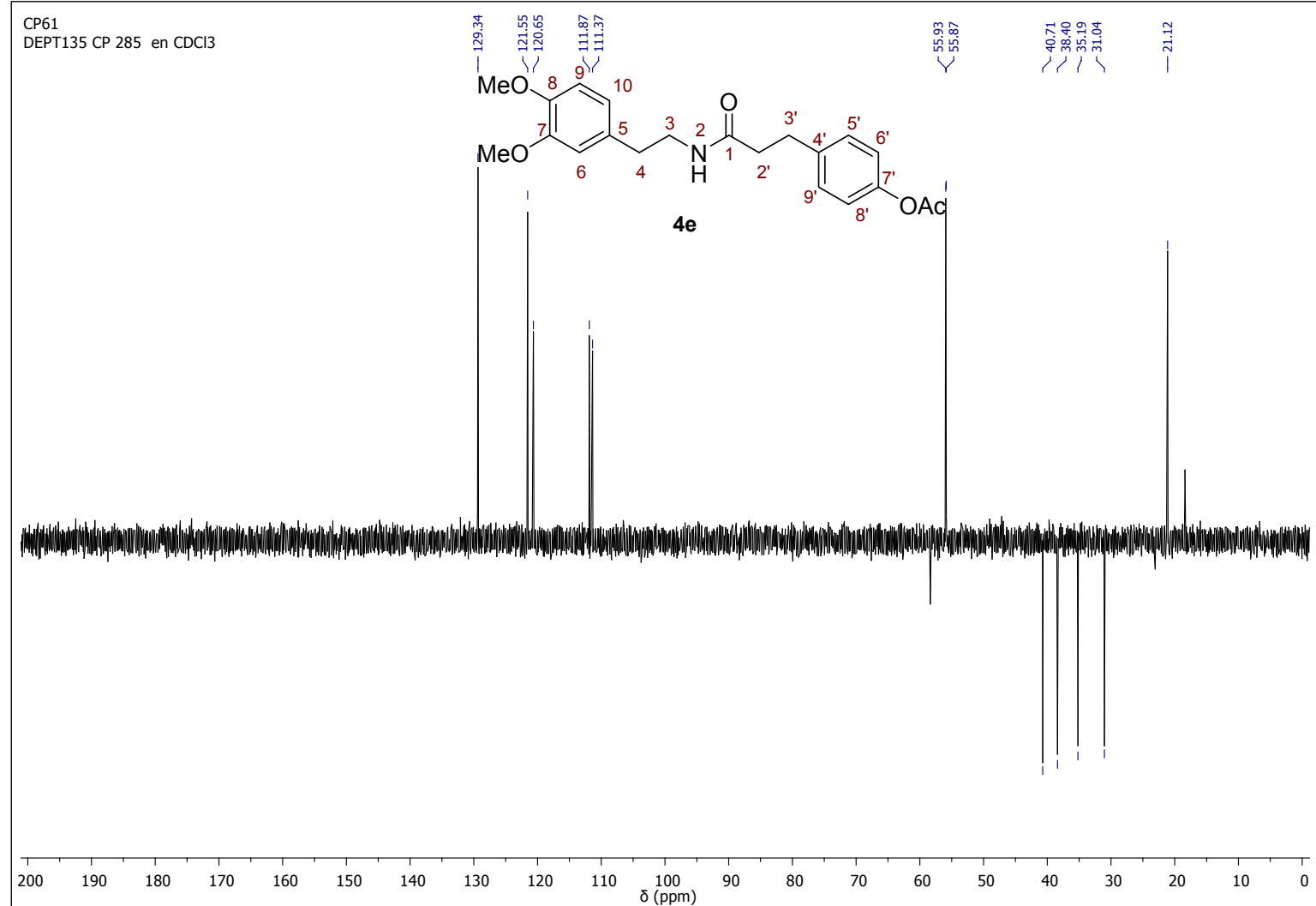

Figure S37. ${ }^{1} \mathrm{H}-\mathrm{NMR}$ spectrum of $N$-(3,4-dimethoxyphenethyl)-3-(4-acetoxy-3-methoxyphenyl)propanamide (4f).

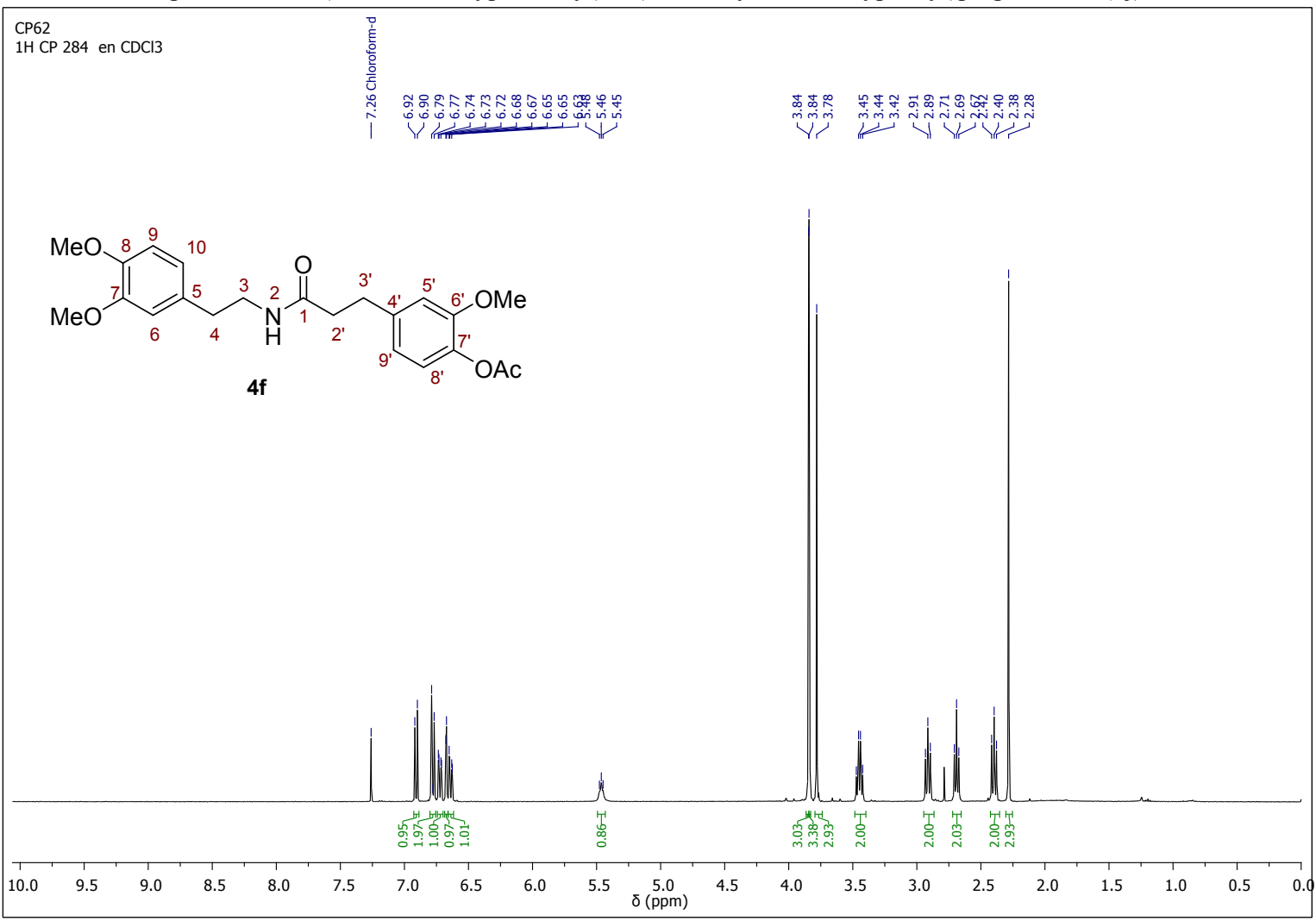


Figure S38. ${ }^{13} \mathrm{C}-\mathrm{NMR}$ spectrum of $N$-(3,4-dimethoxyphenethyl)-3-(4-acetoxy-3-methoxyphenyl)propanamide (4f).

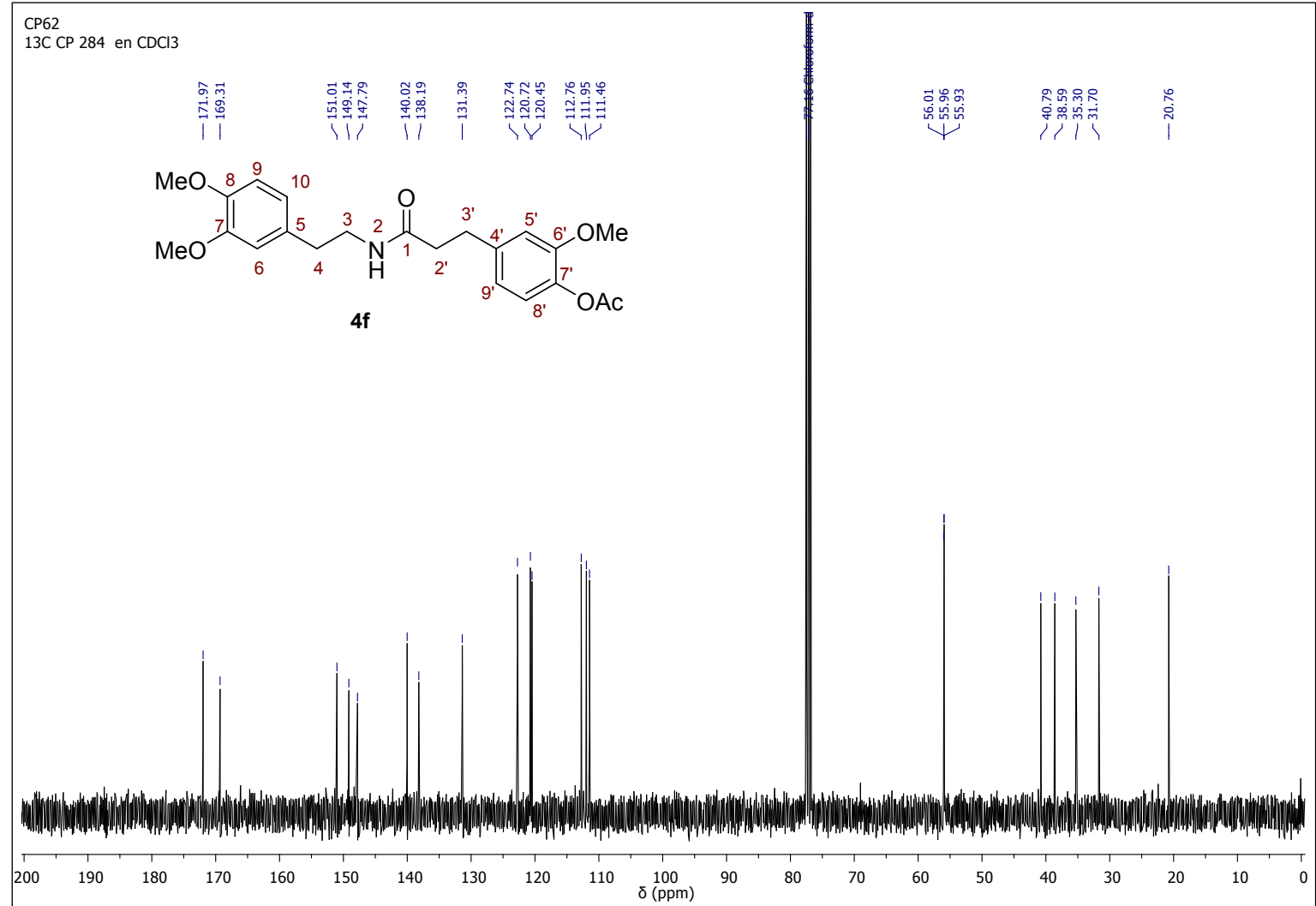

Figure S39. DEPT-135 spectrum of $N$-(3,4-dimethoxyphenethyl)-3-(4-acetoxy-3-methoxyphenyl)propanamide (4f).

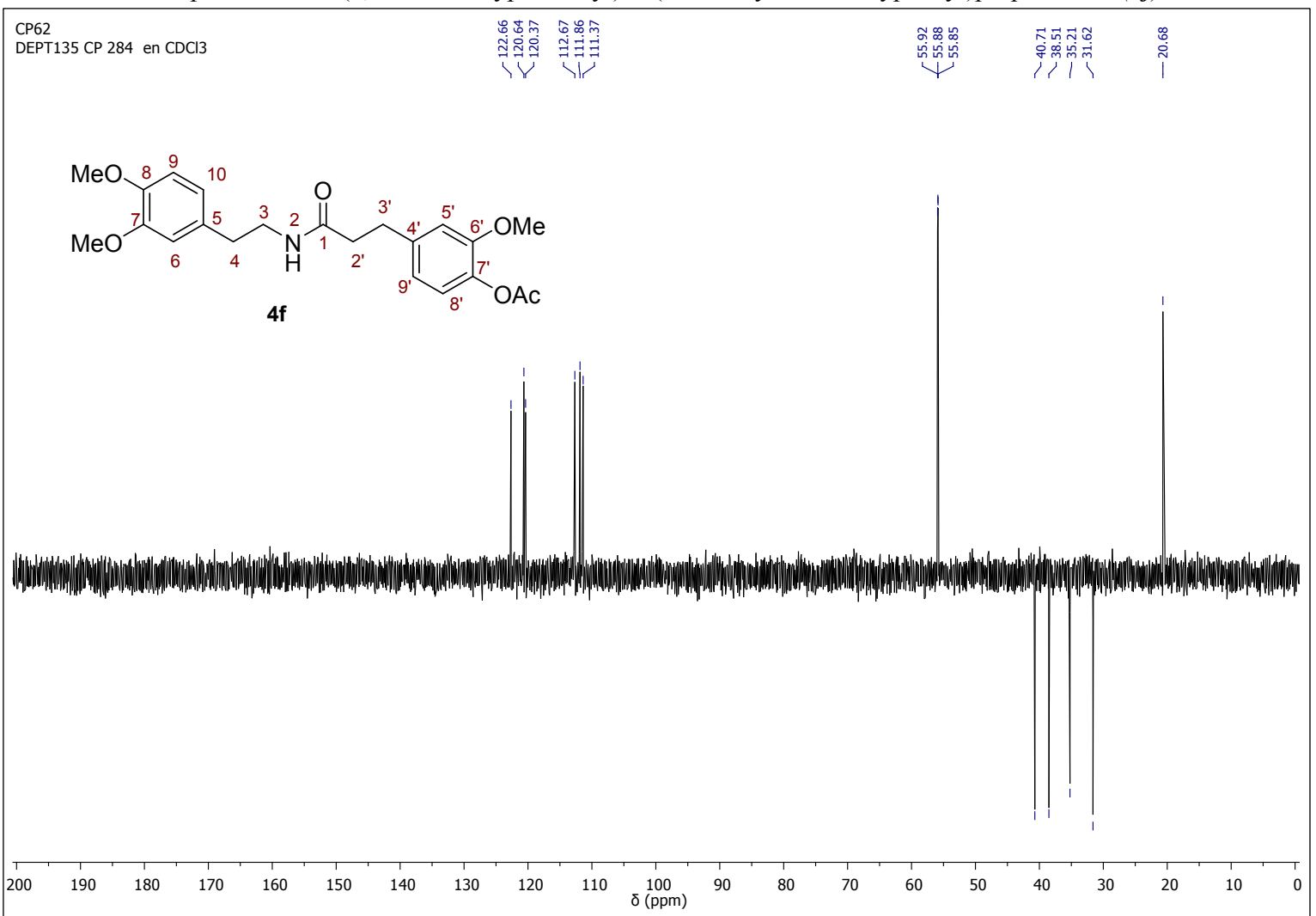


Figure S40. ${ }^{1} \mathrm{H}-\mathrm{NMR}$ spectrum of $N$-(3,4-dimethoxyphenethyl)-3-(3-acetoxy-4-methoxyphenyl)propanamide (4g).

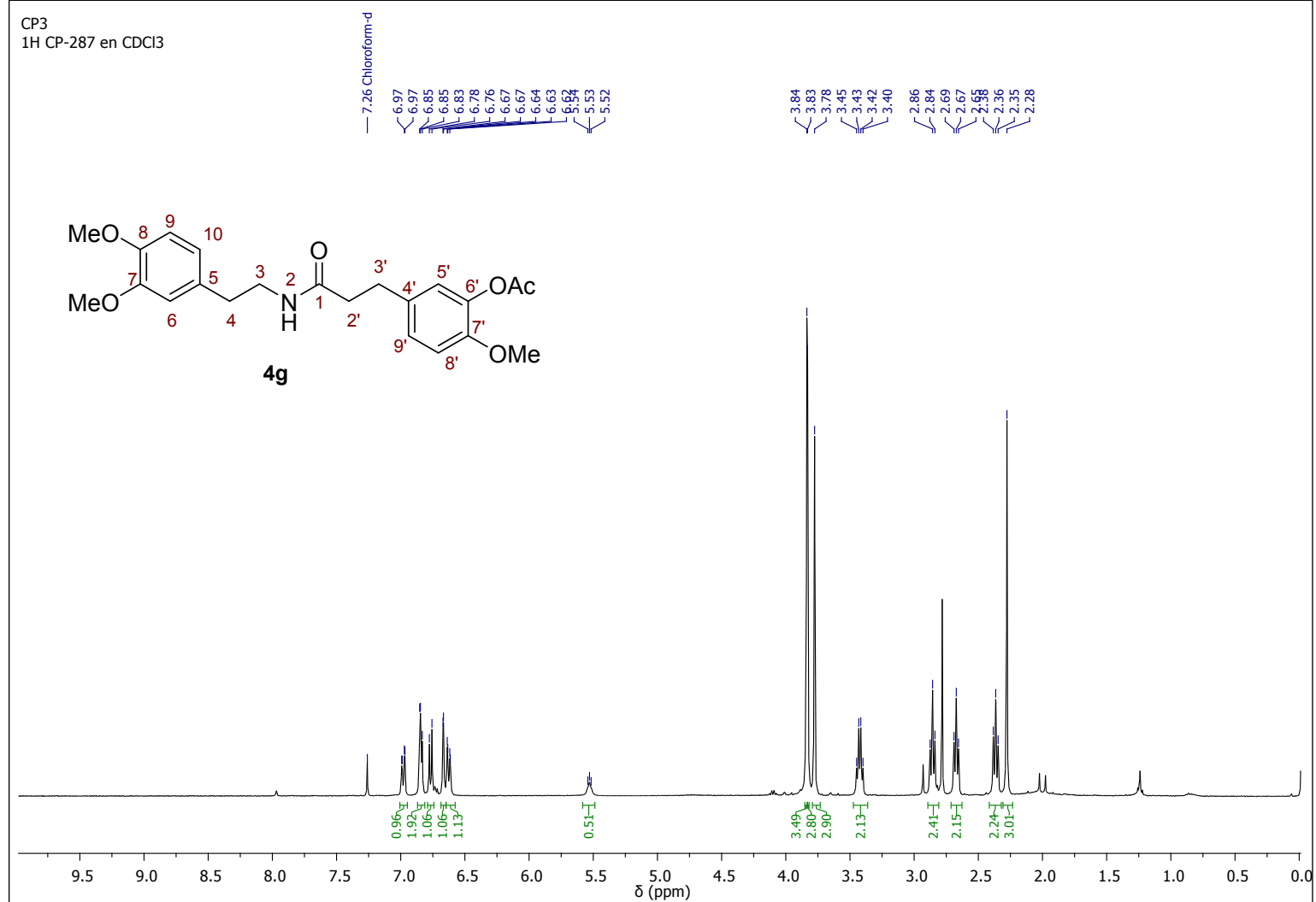

Figure S41. ${ }^{13} \mathrm{C}-\mathrm{NMR}$ spectrum of $N$-(3,4-dimethoxyphenethyl)-3-(3-acetoxy-4-methoxyphenyl)propanamide (4g).

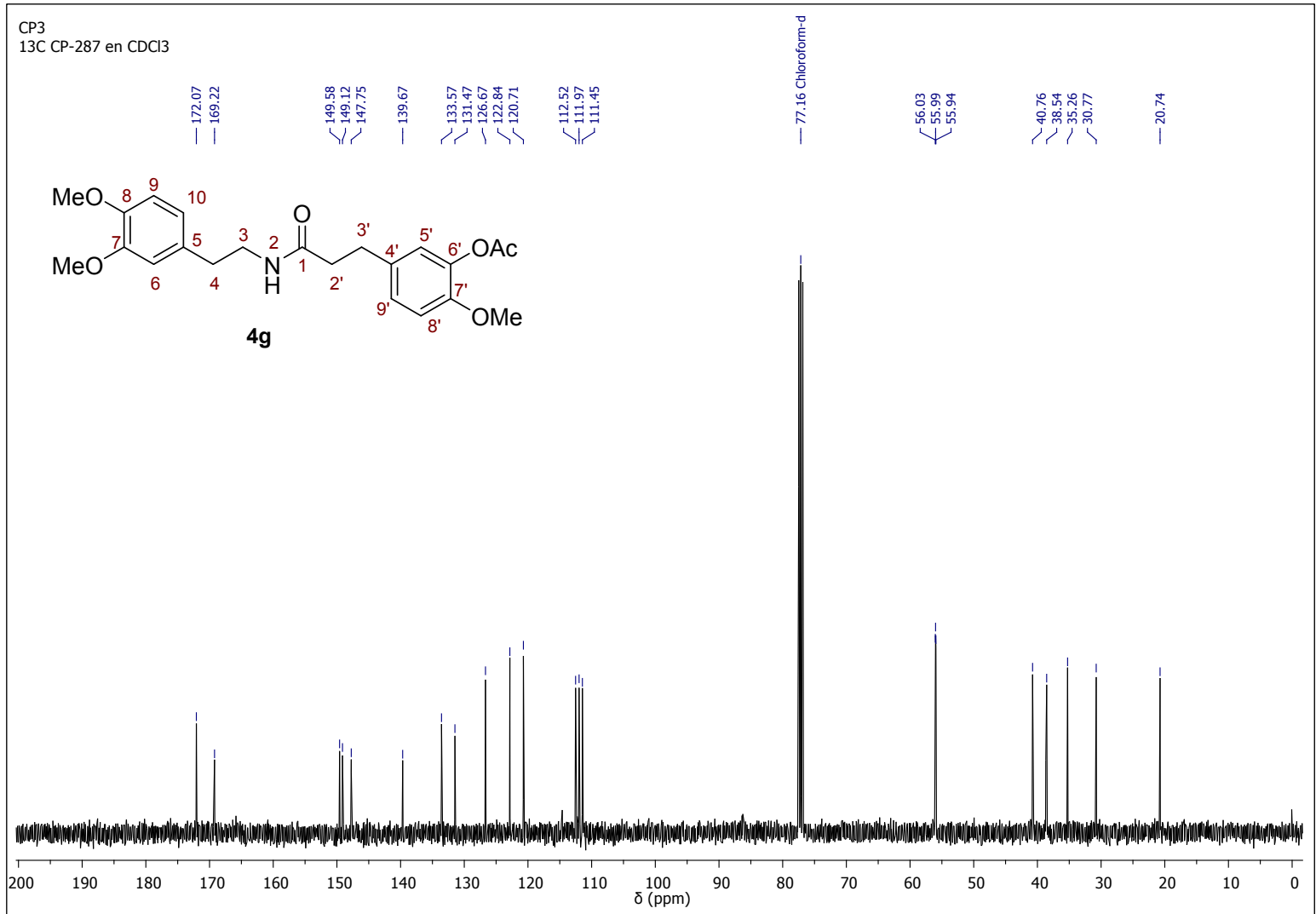


Figure S42. DEPT-135 spectrum of $N$-(3,4-dimethoxyphenethyl)-3-(3-acetoxy-4-methoxyphenyl)propanamide (4g).

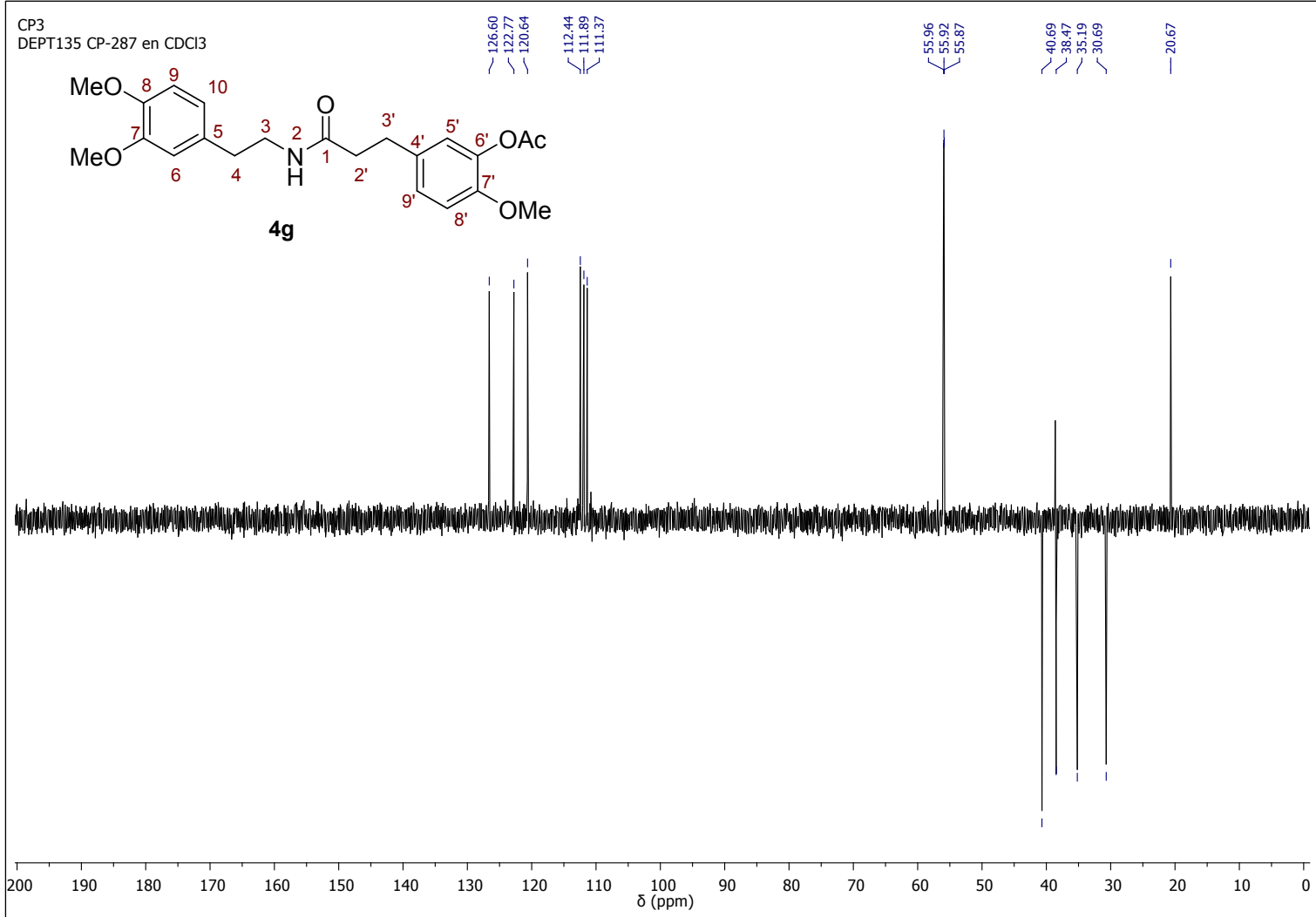

Figure S43. ${ }^{1} \mathrm{H}-\mathrm{NMR}$ spectrum of $N$-(3,4-dimethoxyphenethyl)-3-(4-acetoxy-3,5-dimethoxyphenyl)propanamide (4h).

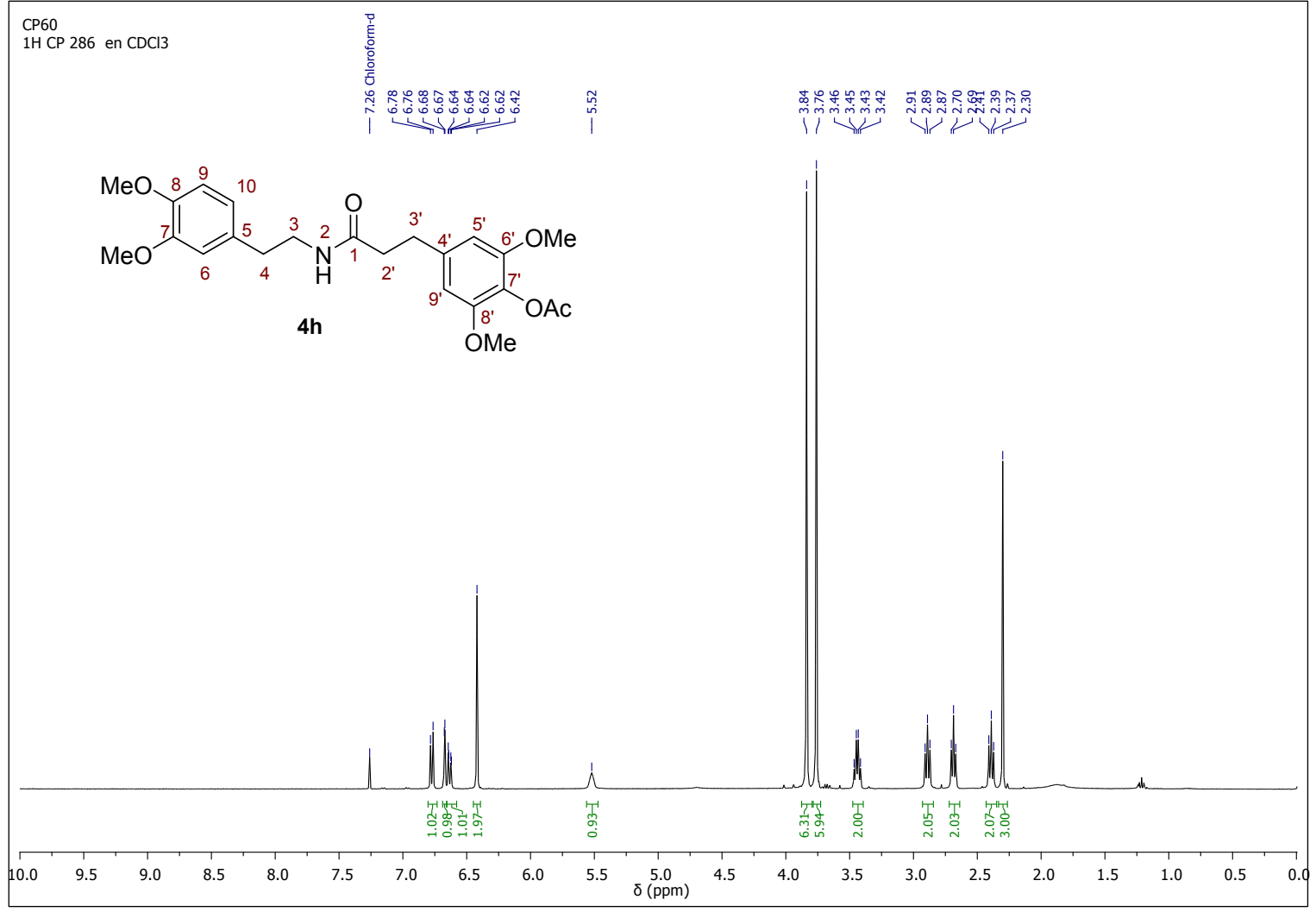


Figure S44. ${ }^{13} \mathrm{C}-\mathrm{NMR}$ spectrum of $N$-(3,4-dimethoxyphenethyl)-3-(4-acetoxy-3,5-dimethoxyphenyl)propanamide (4h).

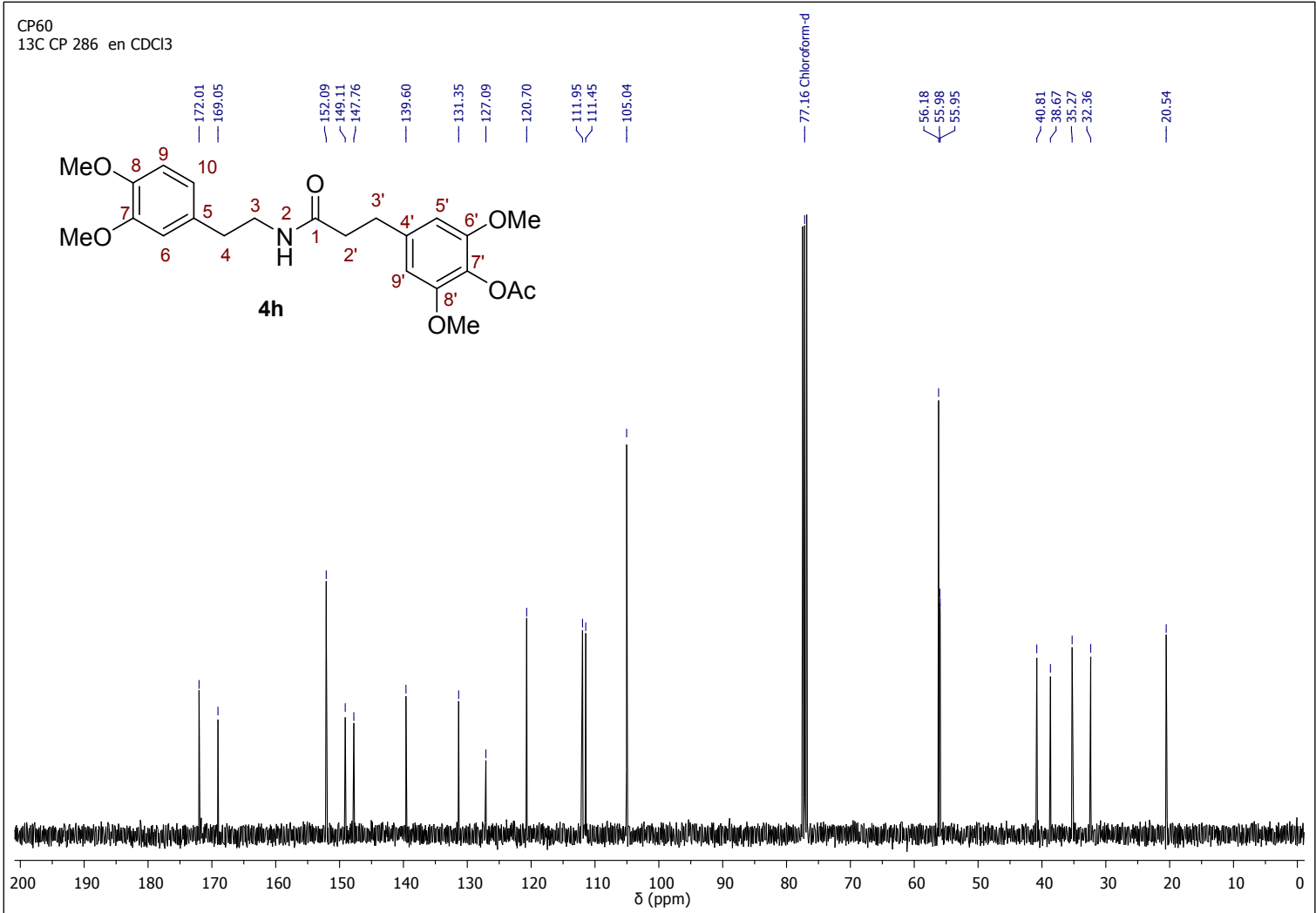

Figure S45. DEPT-135 spectrum of $N$-(3,4-dimethoxyphenethyl)-3-(4-acetoxy-3,5-dimethoxyphenyl)propanamide (4h).

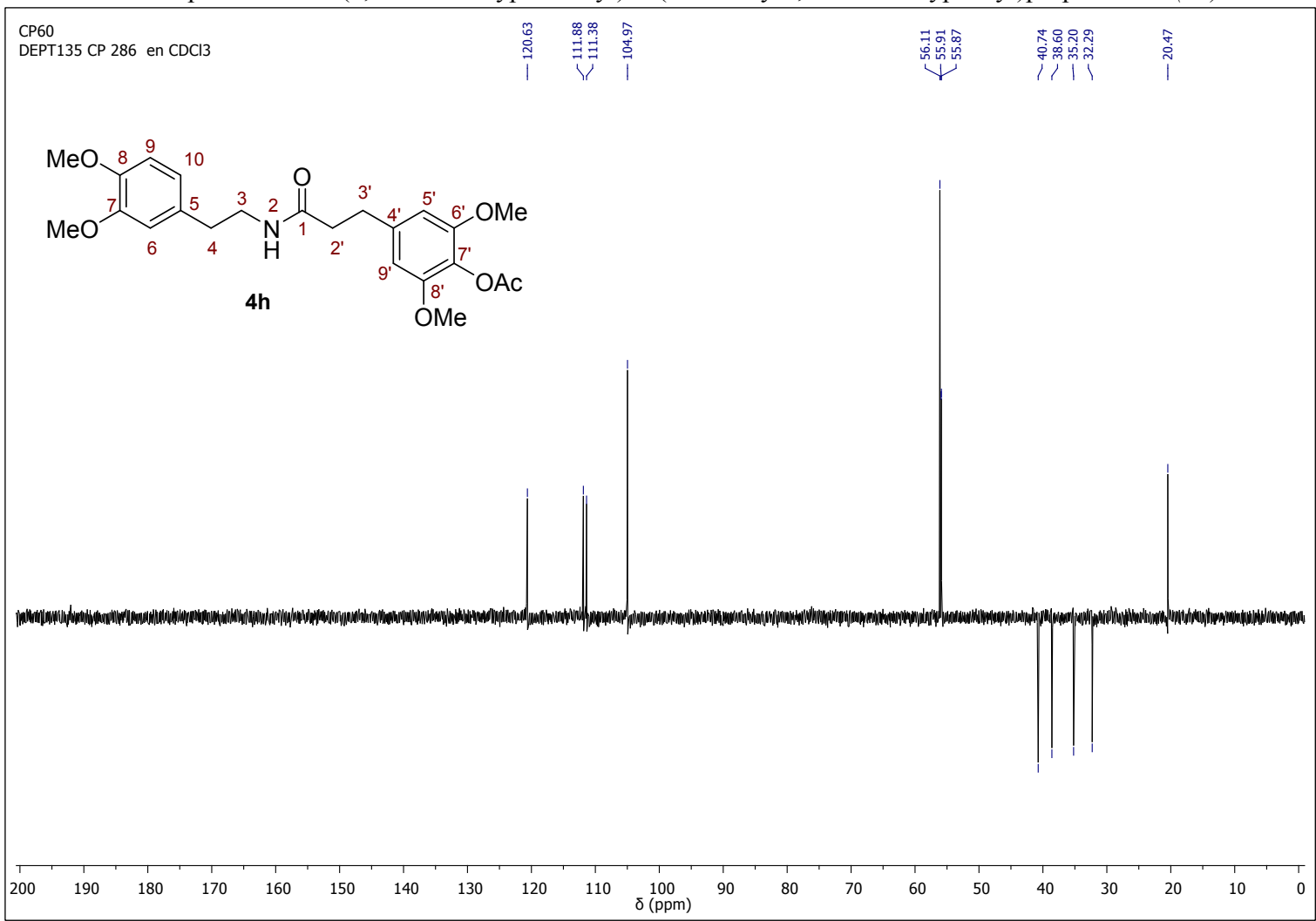




\section{Copies of ${ }^{1} \mathrm{H}$ NMR, ${ }^{13} \mathrm{C}$ NMR and DEPT-135 charts of 1-phenethyl-3,4-dihydroisoquinolines 5a-i}

Figure S46. ${ }^{1} \mathrm{H}$-NMR spectrum of 6,7-dimethoxy-1-phenethyl-3,4-dihydroisoquinoline $(\mathbf{5 a})$.

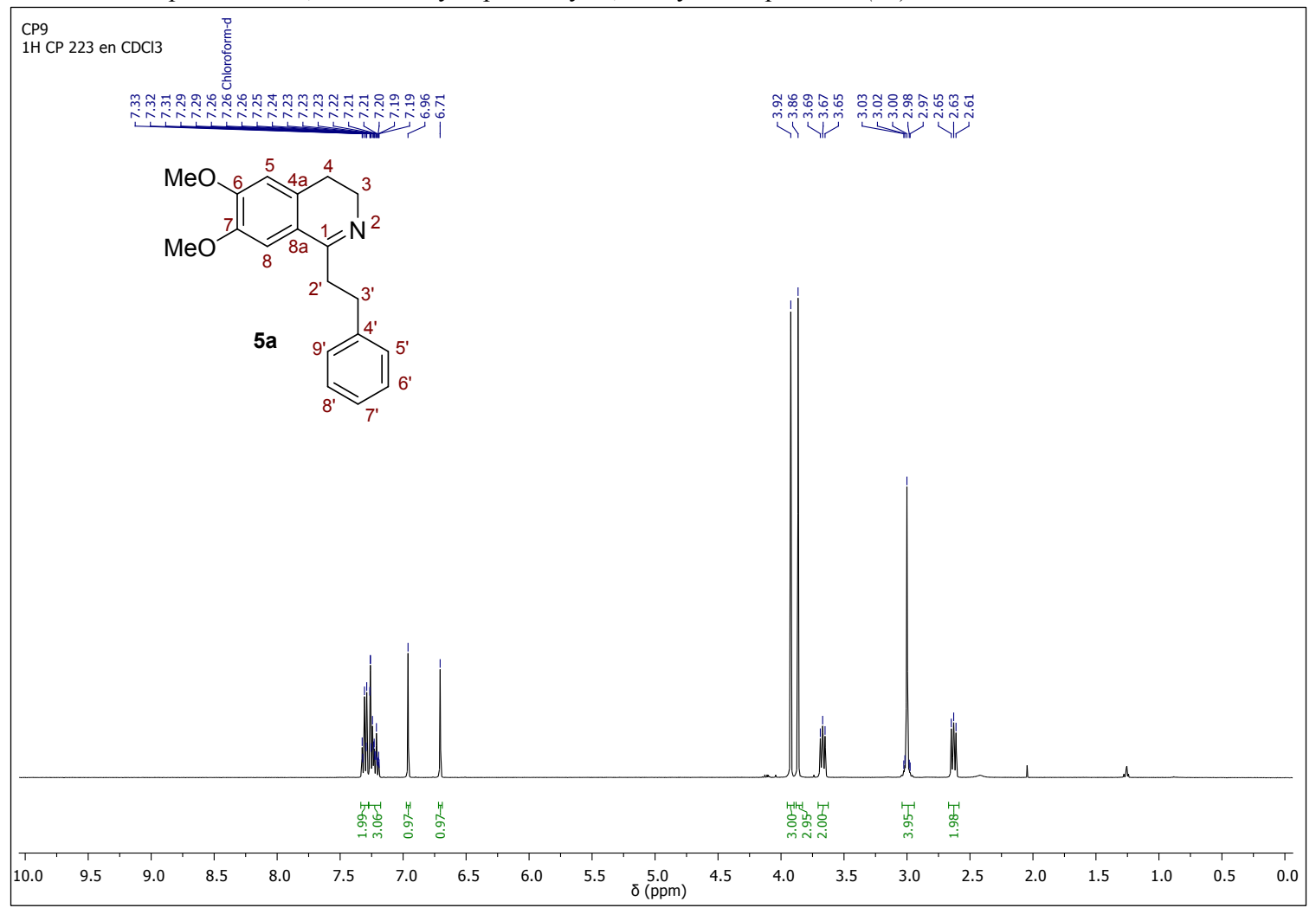

Figure S47. ${ }^{13} \mathrm{C}$-NMR spectrum of 6,7-dimethoxy-1-phenethyl-3,4-dihydroisoquinoline (5a).

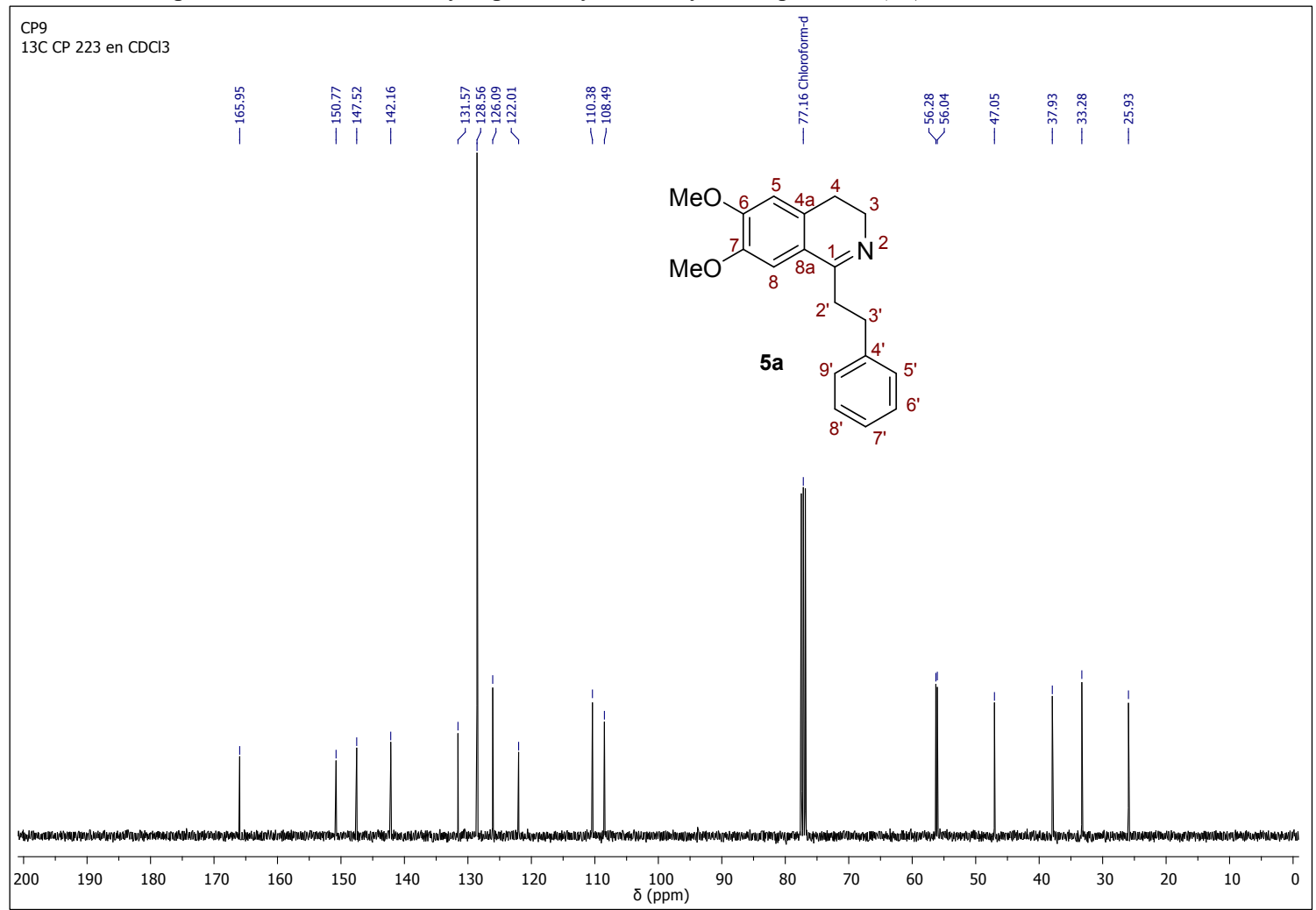


Figure S48. ${ }^{1} \mathrm{H}-\mathrm{NMR}$ spectrum of 6,7-dimethoxy-1-(4-methoxyphenethyl)-3,4-dihydroisoquinoline (5b).

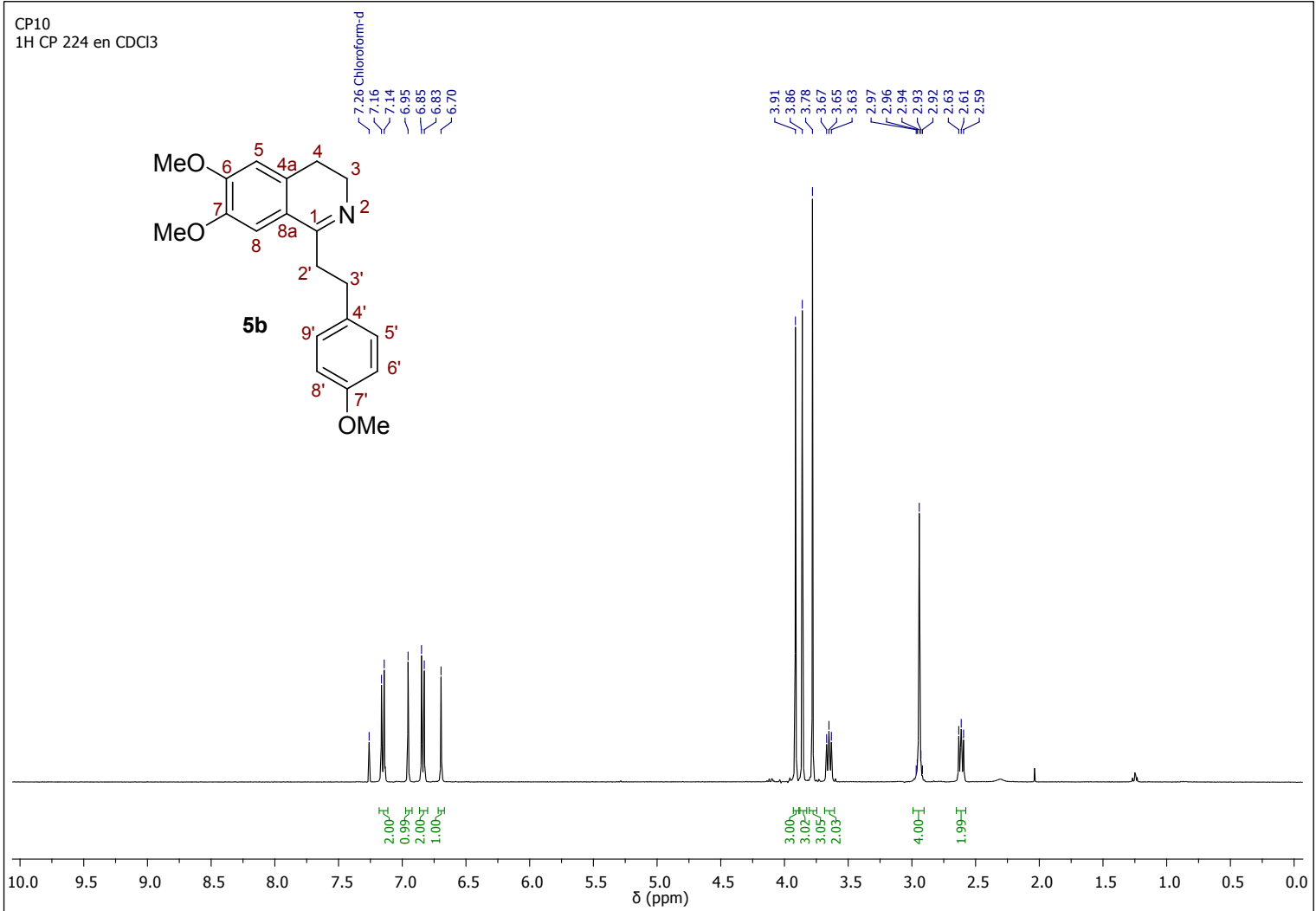

Figure S49. ${ }^{13} \mathrm{C}-\mathrm{NMR}$ spectrum of 6,7-dimethoxy-1-(4-methoxyphenethyl)-3,4-dihydroisoquinoline (5b).

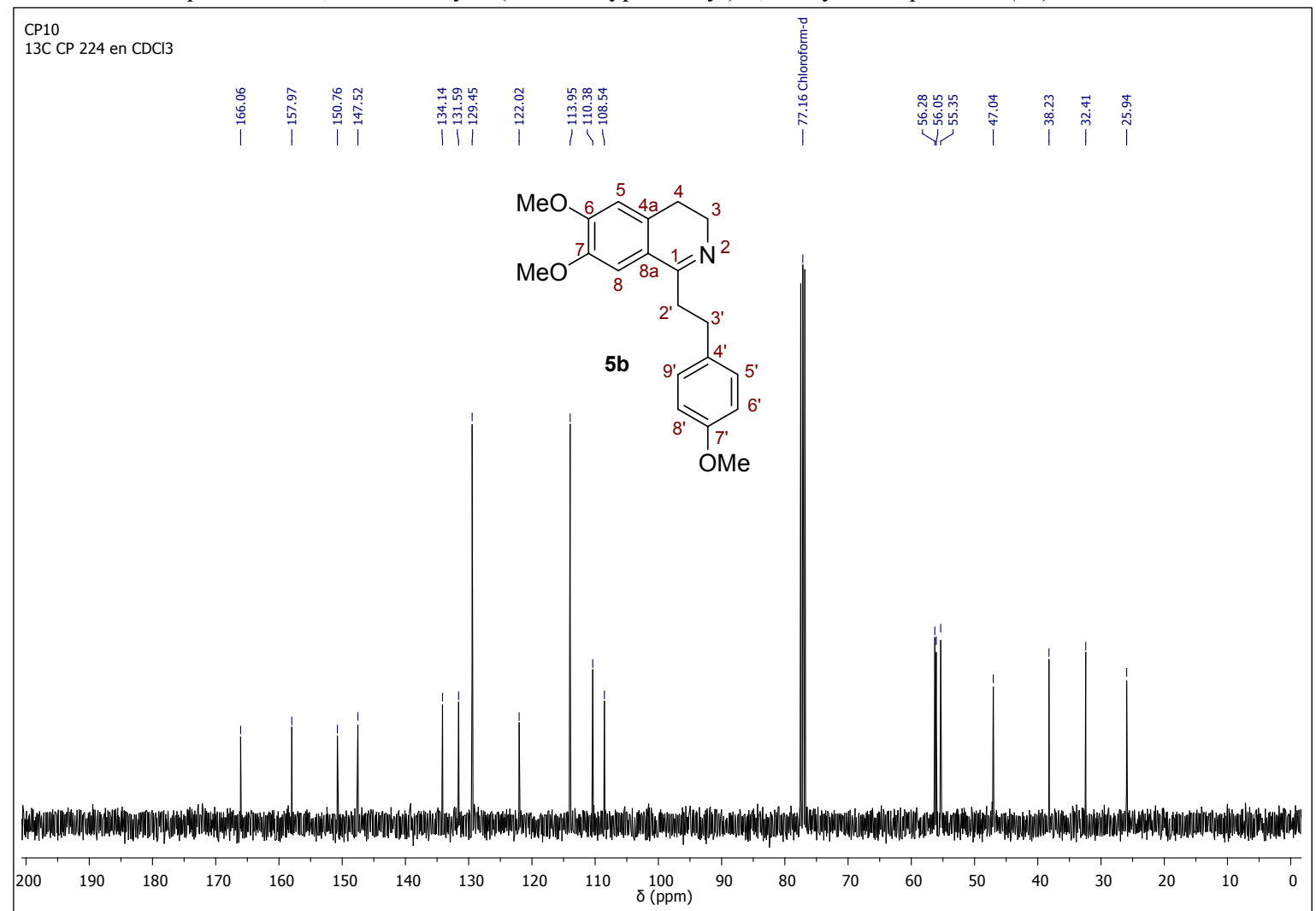


Figure S50. ${ }^{1}$ H-NMR spectrum of 6,7-dimethoxy-1-(3,4-dimethoxyphenethyl)-3,4-dihydroisoquinoline (5c).

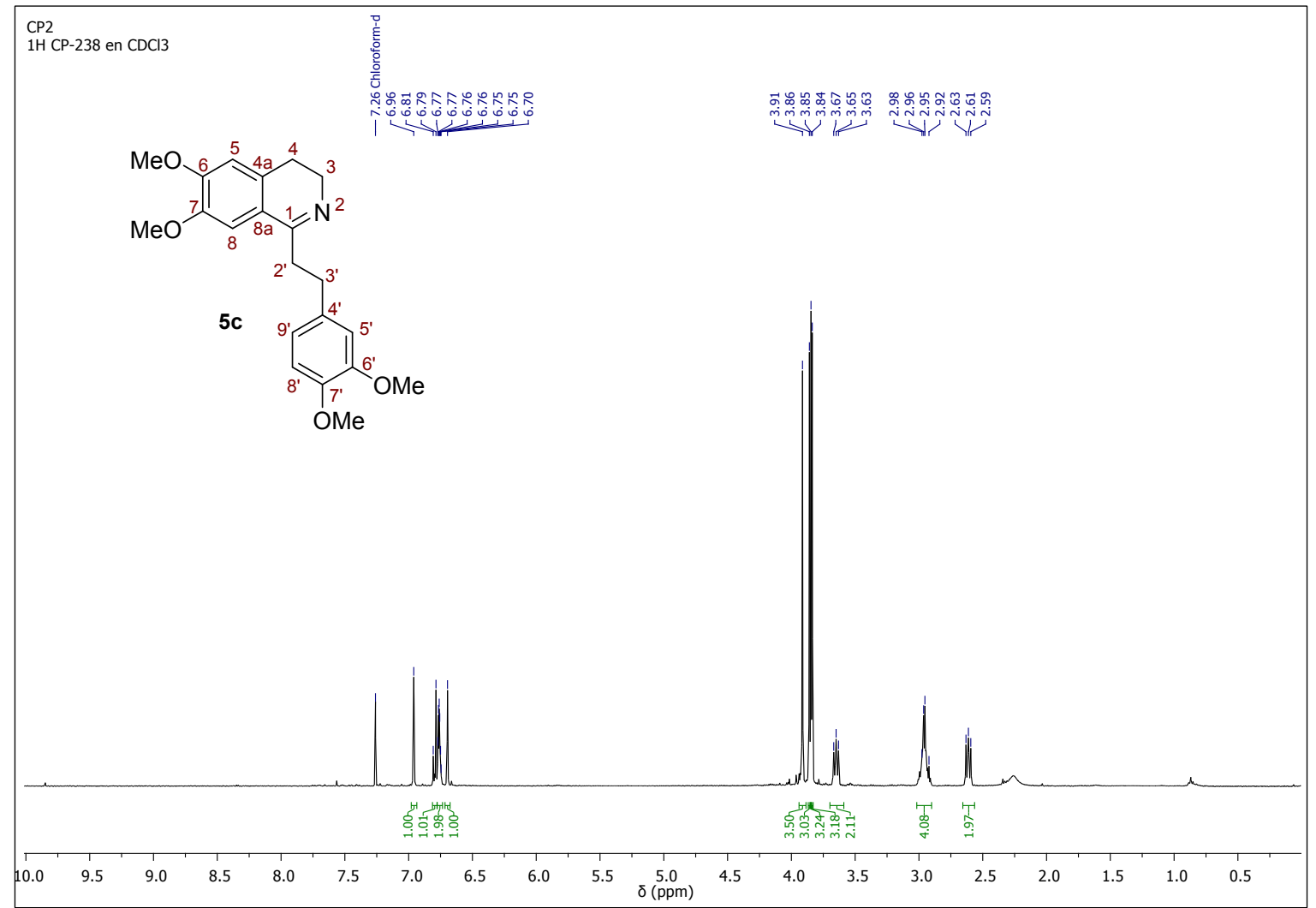

Figure S51. ${ }^{13}$ C-NMR spectrum of 6,7-dimethoxy-1-(3,4-dimethoxyphenethyl)-3,4-dihydroisoquinoline (5c).

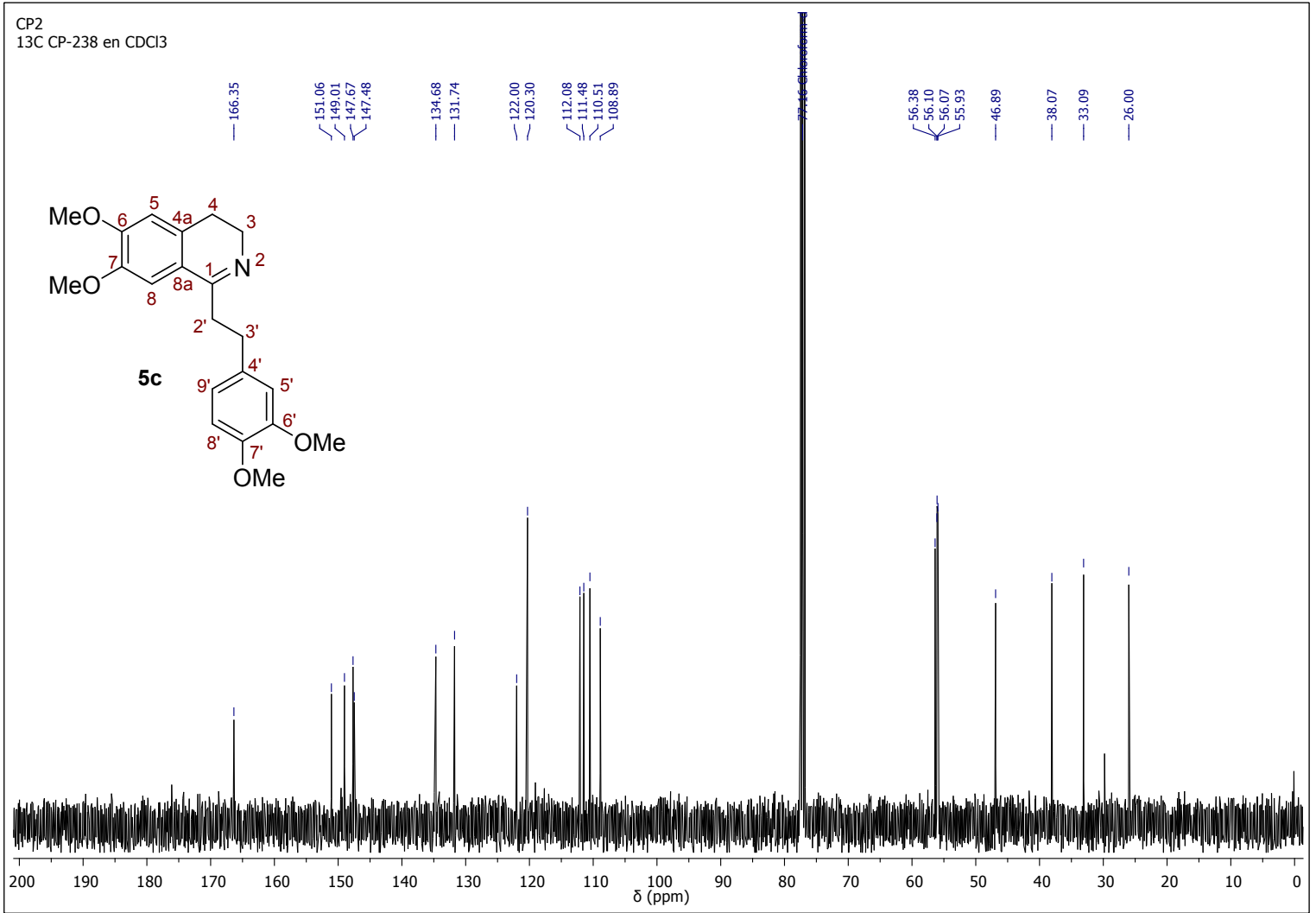


Figure S52. DEPT-135 spectrum of 6,7-dimethoxy-1-(3,4-dimethoxyphenethyl)-3,4-dihydroisoquinoline (5c).

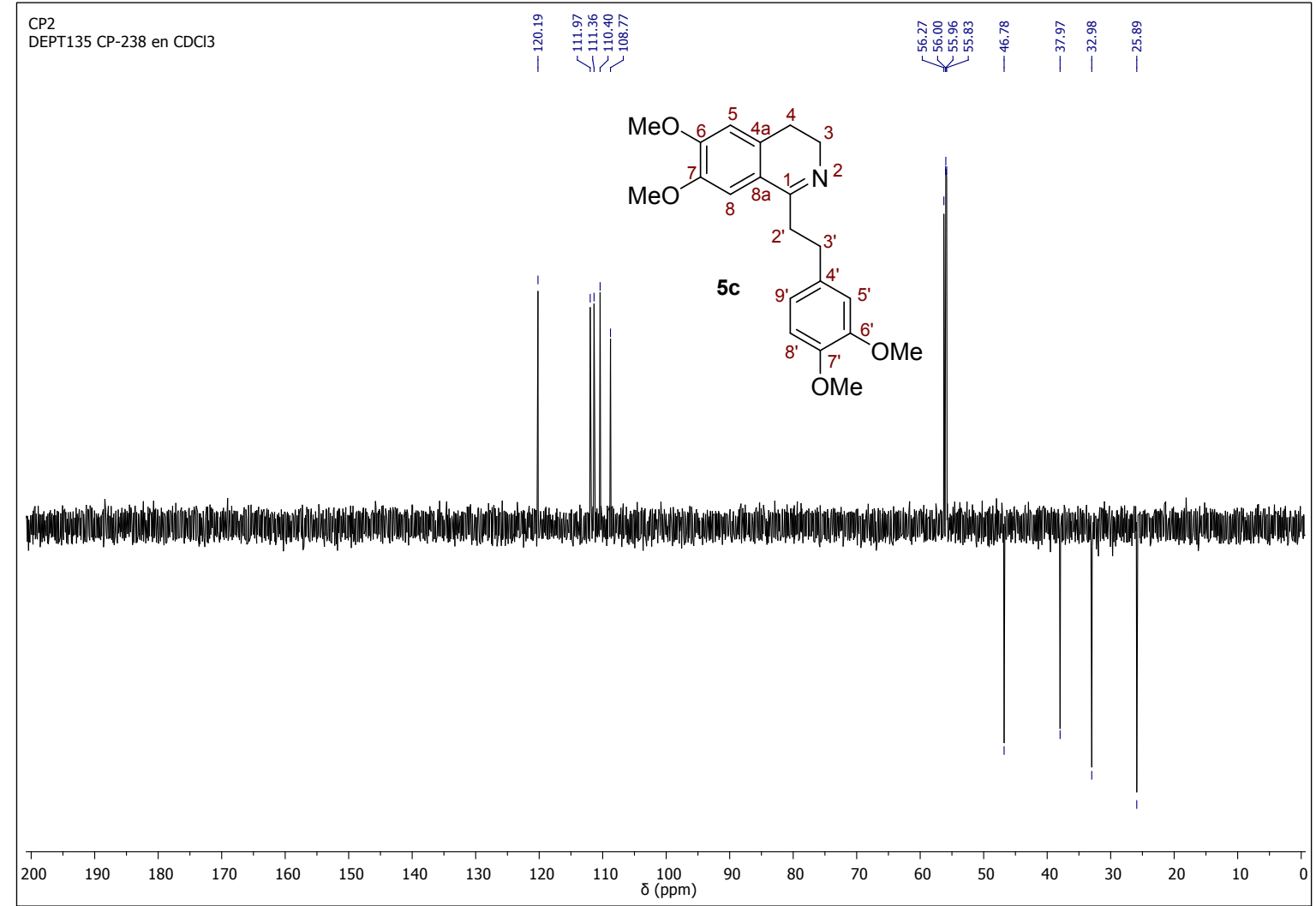

Figure S53. ${ }^{1} \mathrm{H}-\mathrm{NMR}$ spectrum of 6,7-dimethoxy-1-(3,4,5-trimethoxyphenethyl)-3,4-dihydroisoquinoline (5d).

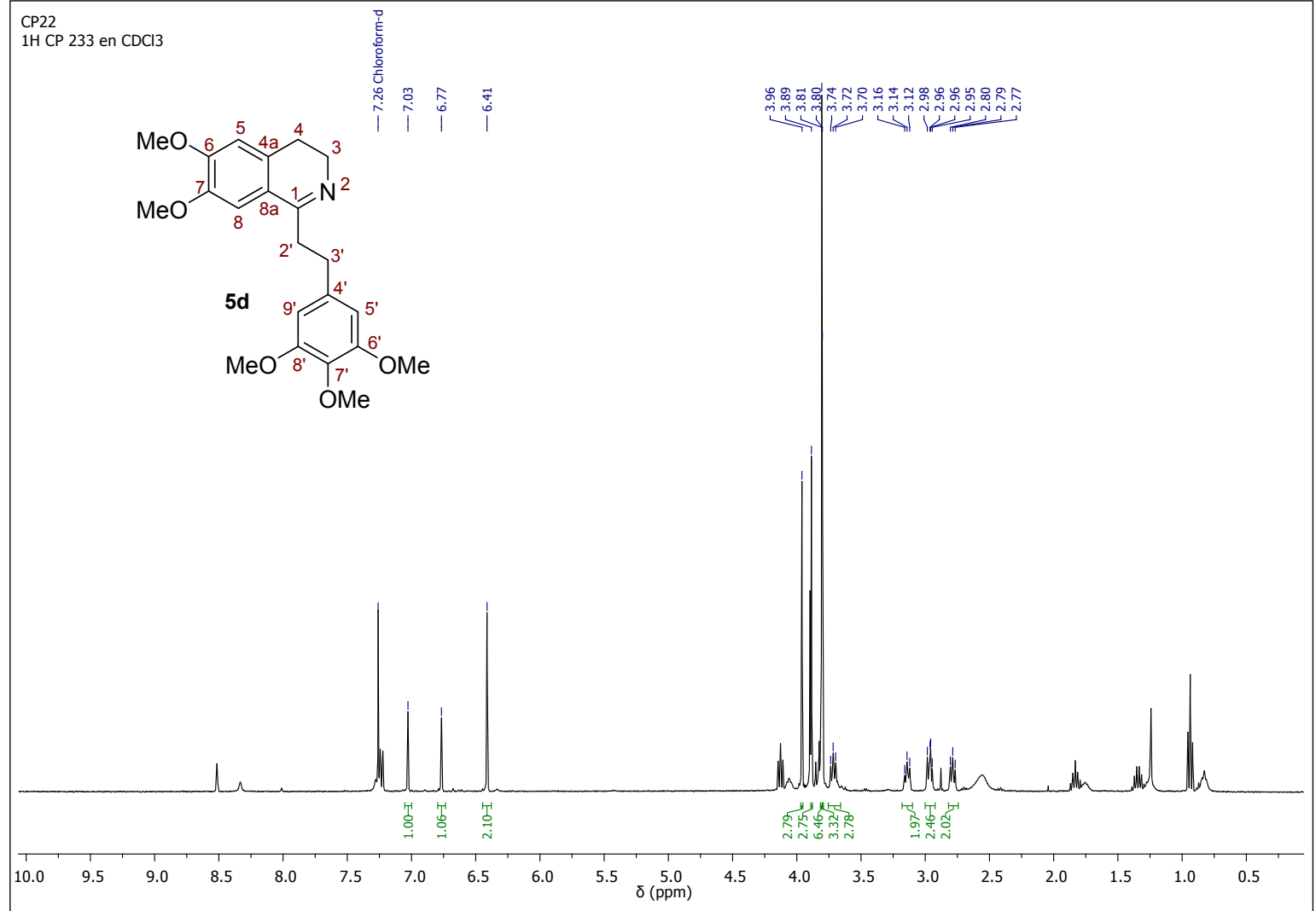


Figure S54. ${ }^{13} \mathrm{C}$-NMR spectrum of 6,7-dimethoxy-1-(3,4,5-trimethoxyphenethyl)-3,4-dihydroisoquinoline (5d).

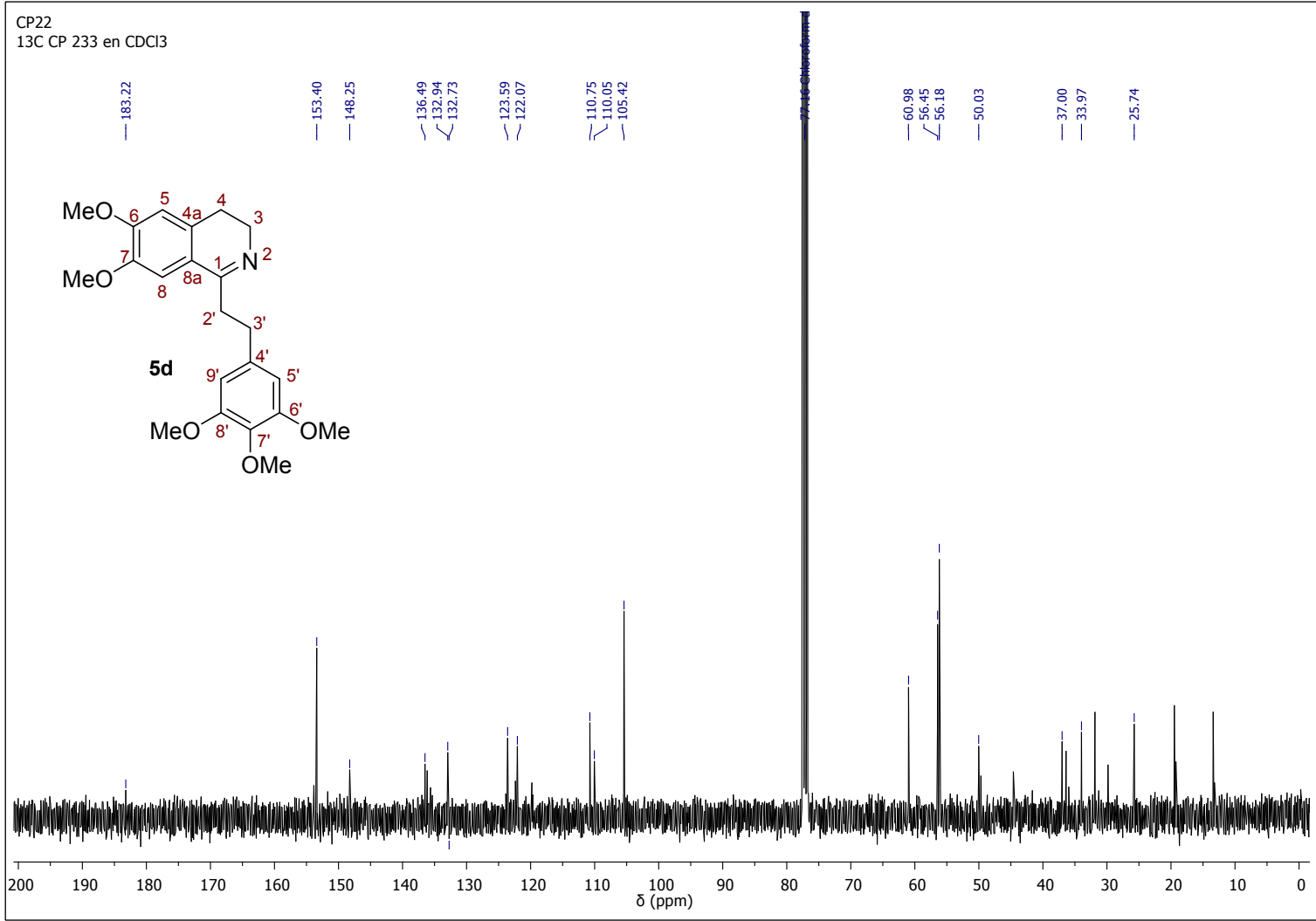

Figure S55. ${ }^{1}$ H-NMR spectrum of 6,7-dimethoxy-1-(3,4-methylendioxyphenethyl)-3,4-dihydroisoquinoline (5e).

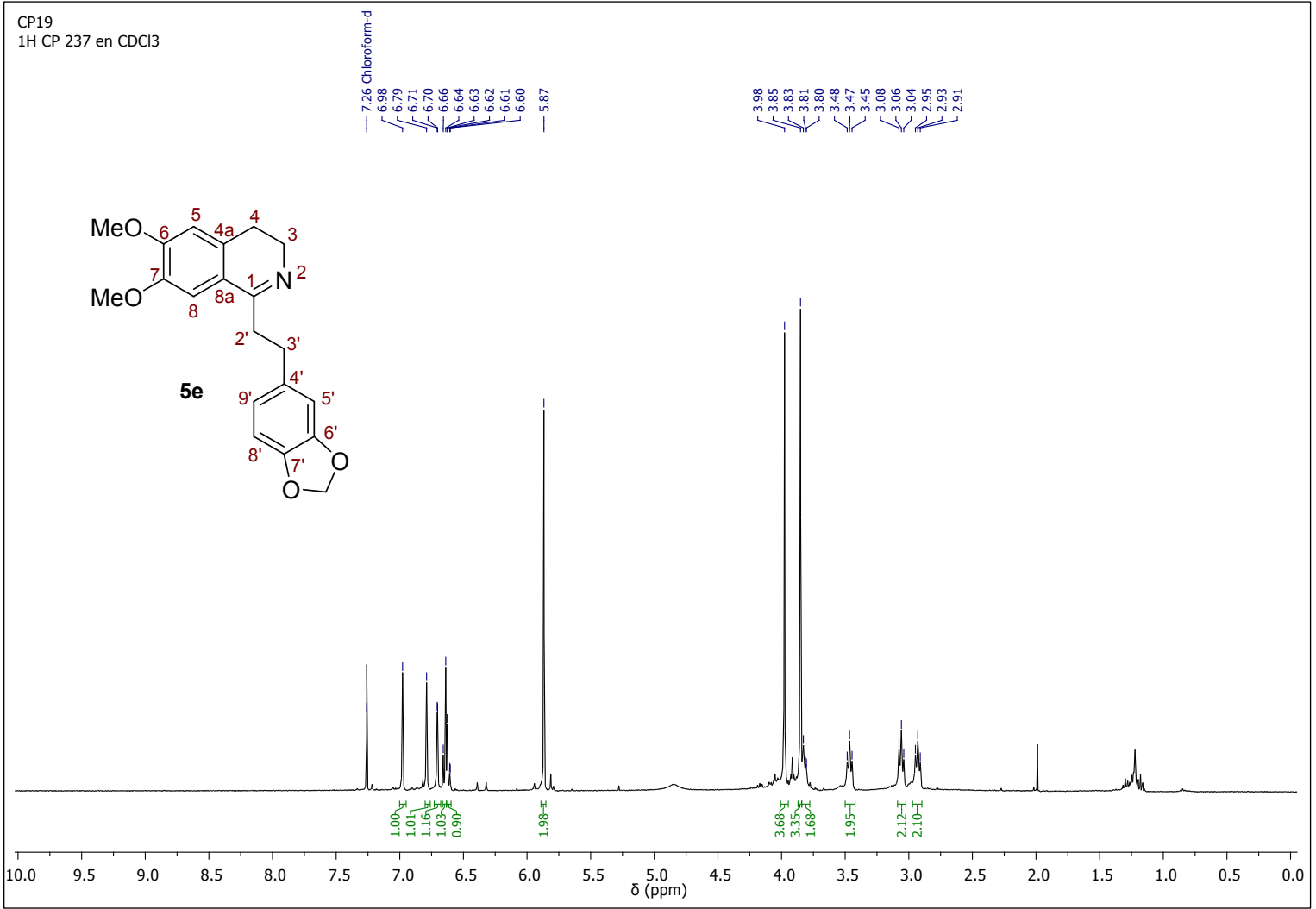


Figure S56. ${ }^{13} \mathrm{C}$-NMR spectrum of 6,7-dimethoxy-1-(3,4-methylendioxyphenethyl)-3,4-dihydroisoquinoline (5e).

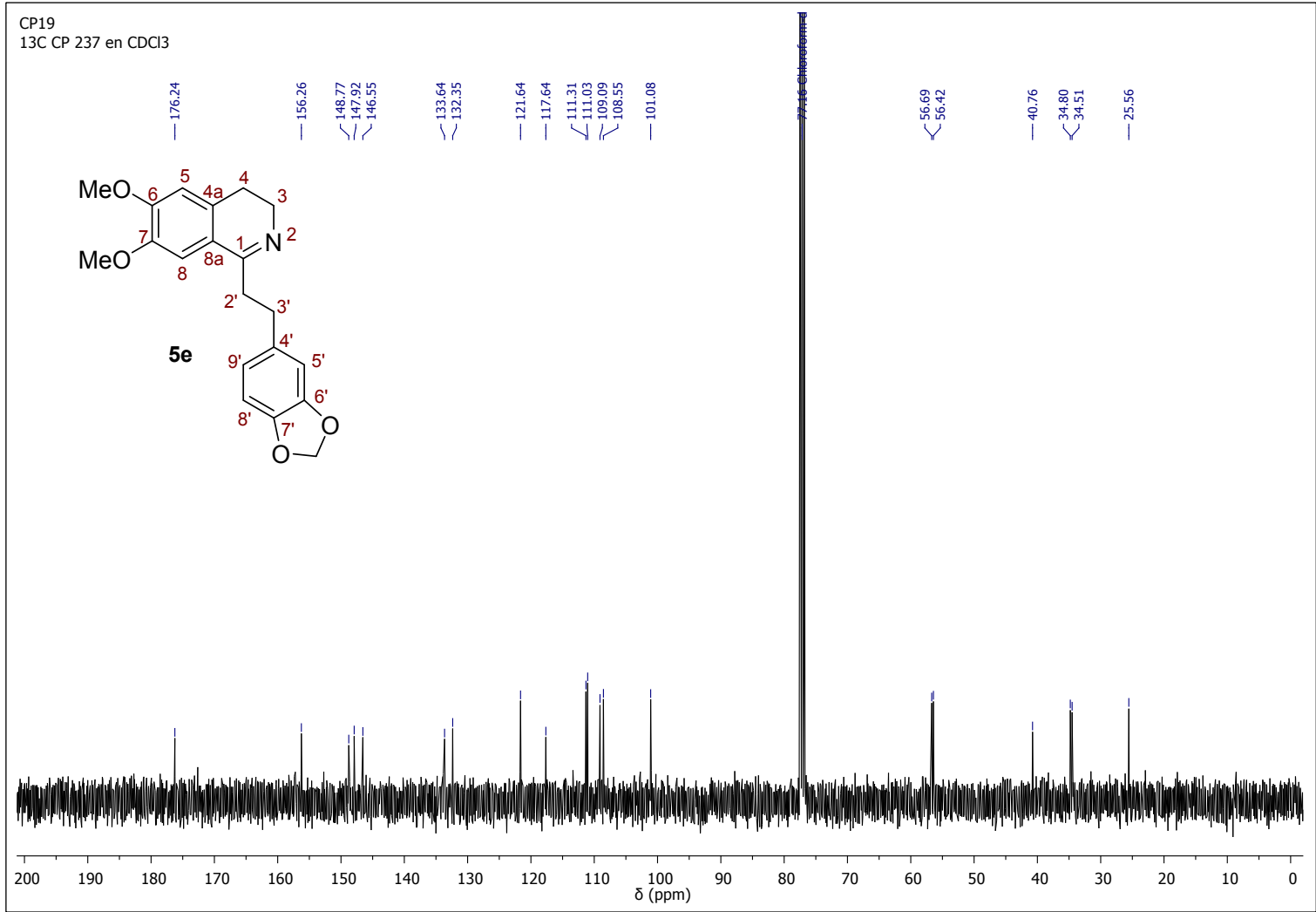

Figure S57. ${ }^{1} \mathrm{H}-\mathrm{NMR}$ spectrum of 6,7-dimethoxy-1-(4-acetoxyphenethyl)-3,4-dihydroisoquinoline (5f).

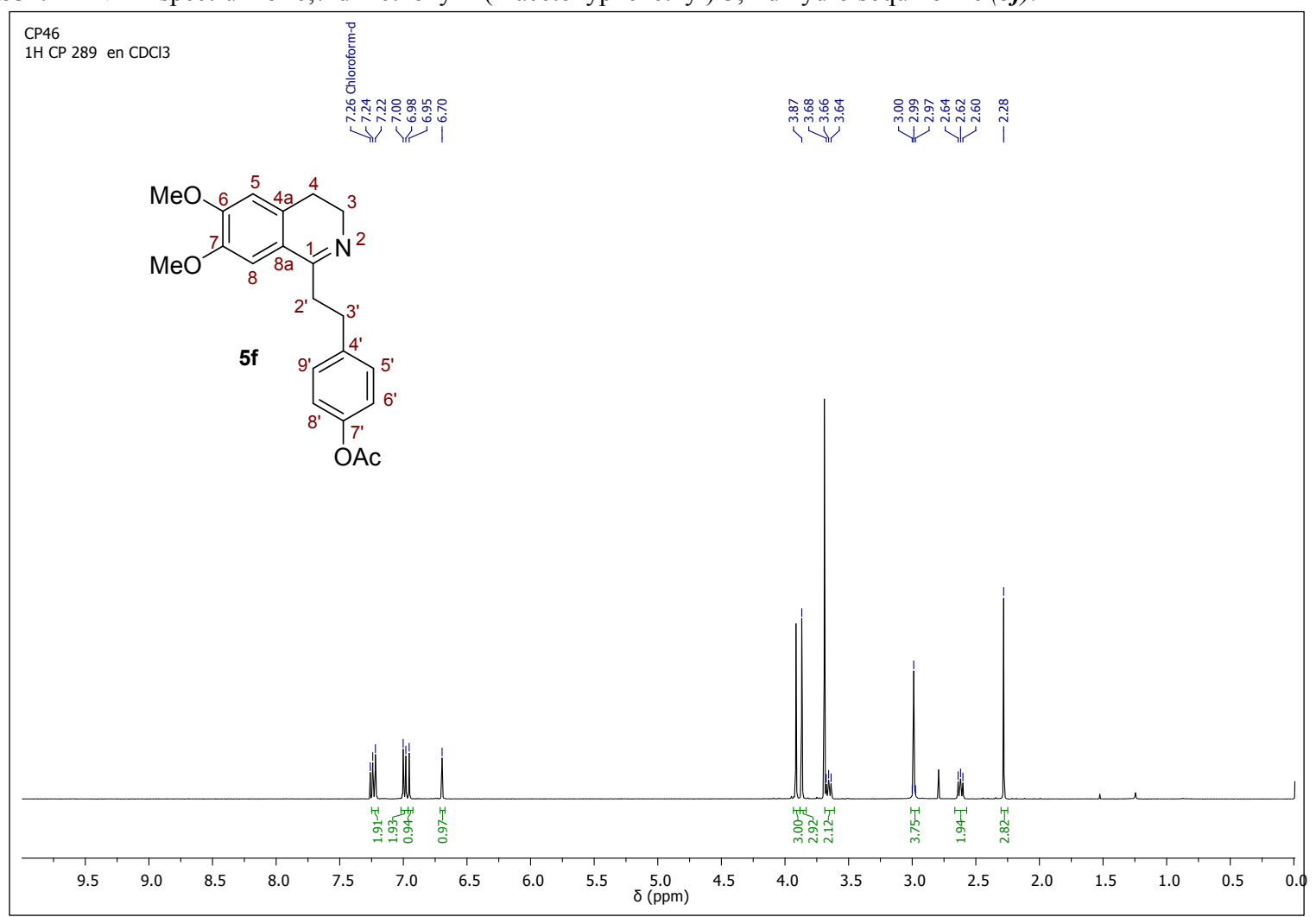


Figure S58. ${ }^{13}$ C-NMR spectrum of 6,7-dimethoxy-1-(4-acetoxyphenethyl)-3,4-dihydroisoquinoline (5f).

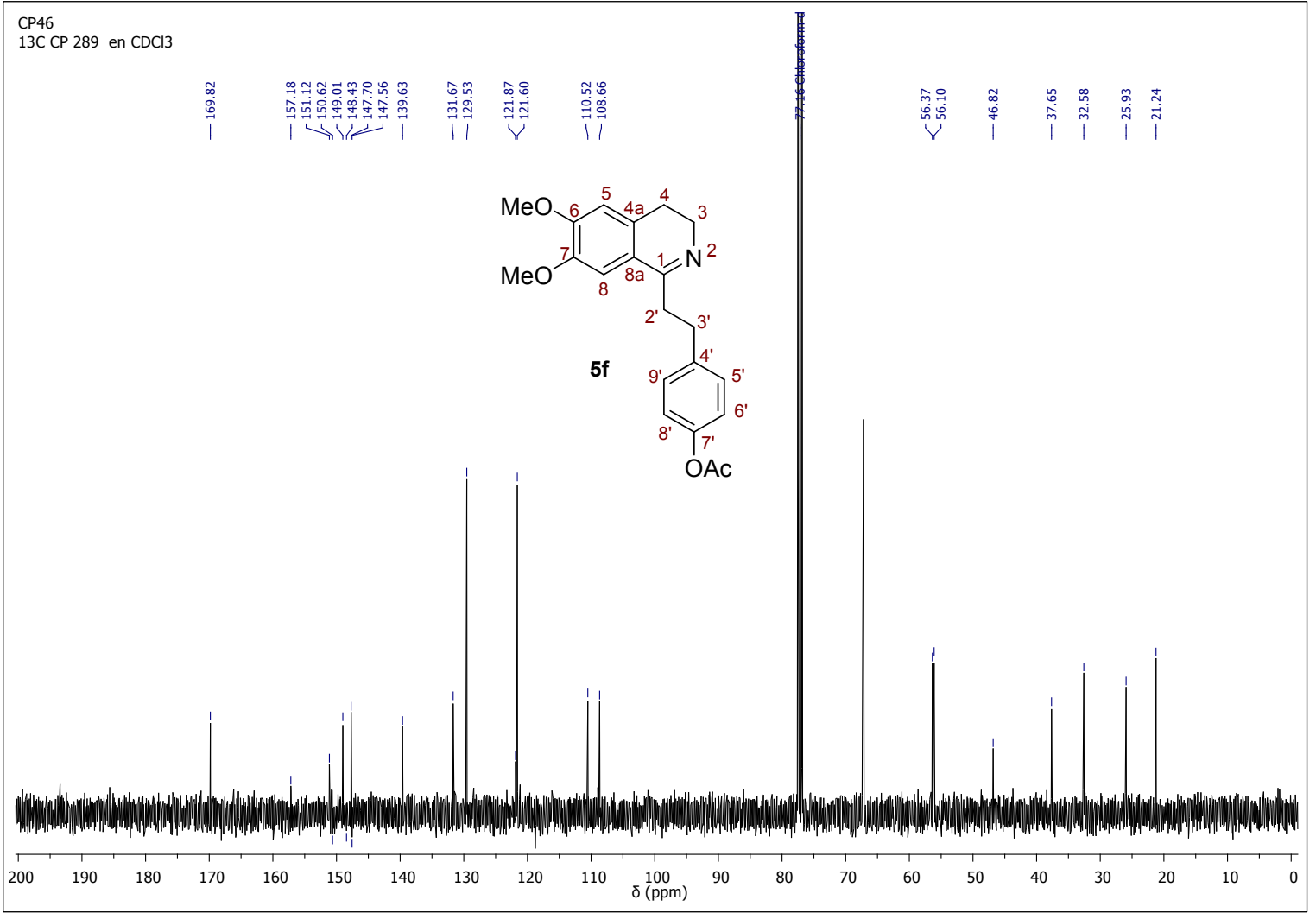

Figure S59. DEPT-135 spectrum of 6,7-dimethoxy-1-(4-acetoxyphenethyl)-3,4-dihydroisoquinoline (5f).

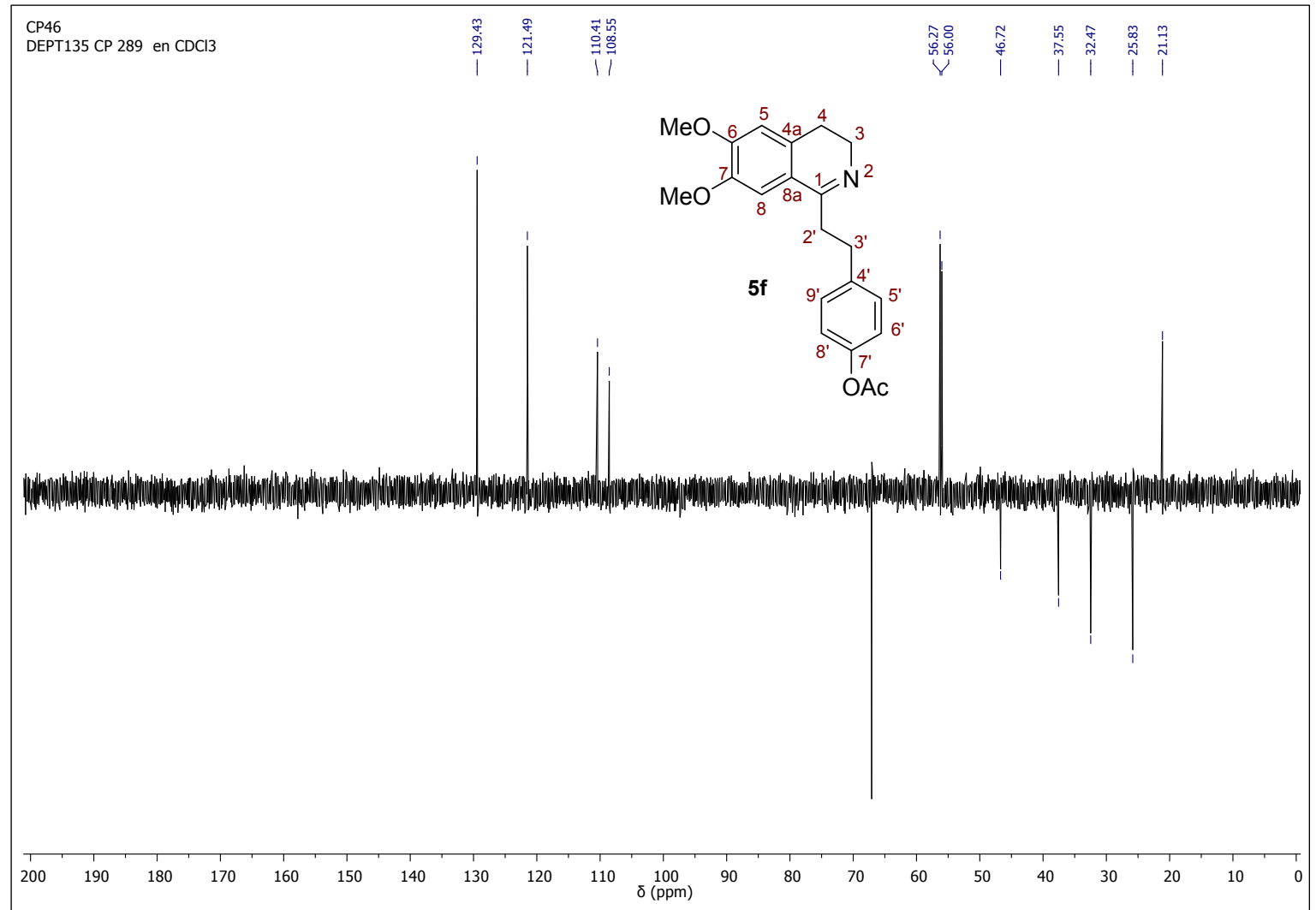


Figure S60. ${ }^{1}$ H-NMR spectrum of 6,7-dimethoxy-1-(4-acetoxy-3-methoxyphenethyl)-3,4-dihydroisoquinoline (5g).

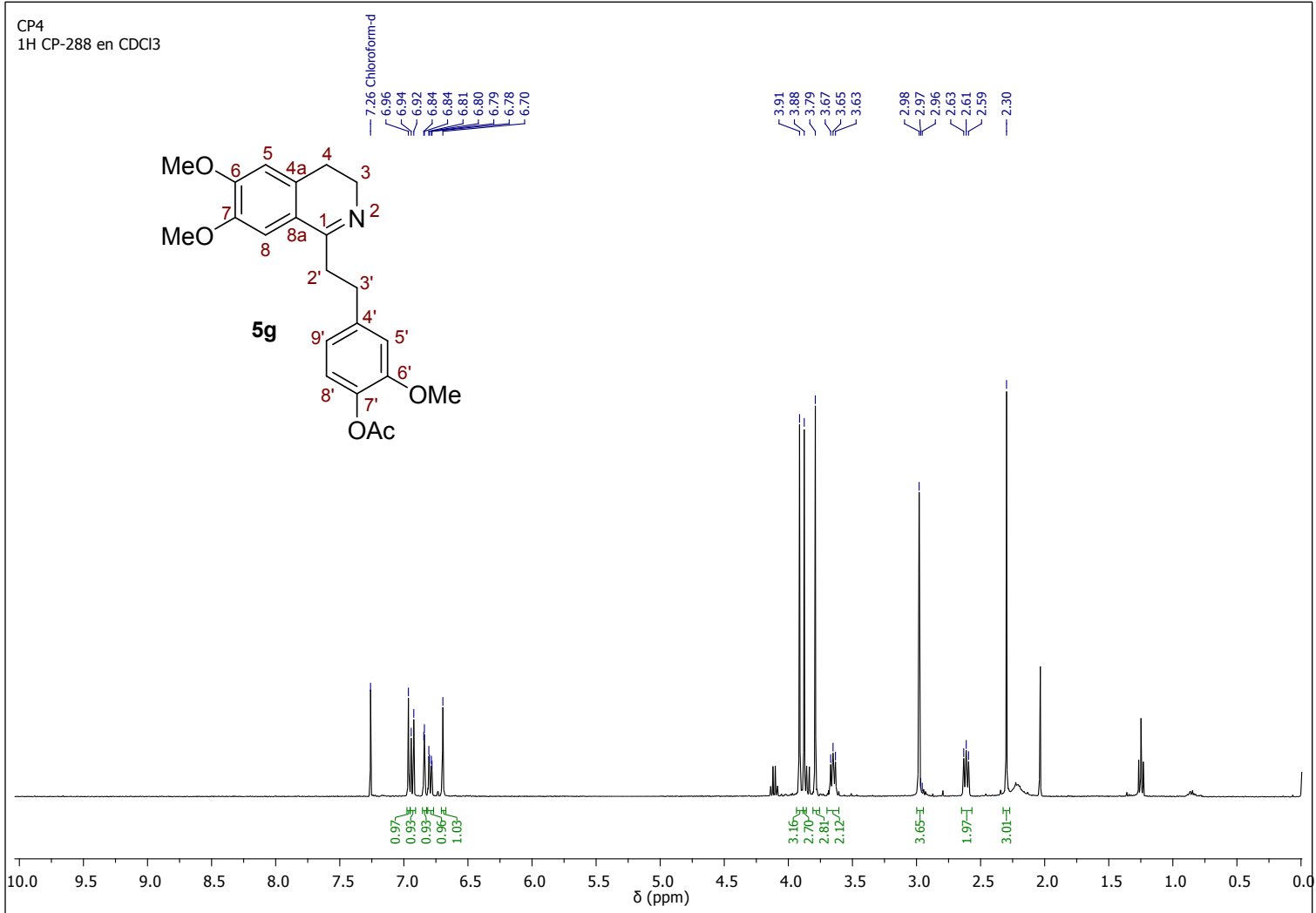

Figure S61. ${ }^{13} \mathrm{C}$-NMR spectrum of 6,7-dimethoxy-1-(4-acetoxy-3-methoxyphenethyl)-3,4-dihydroisoquinoline (5g).

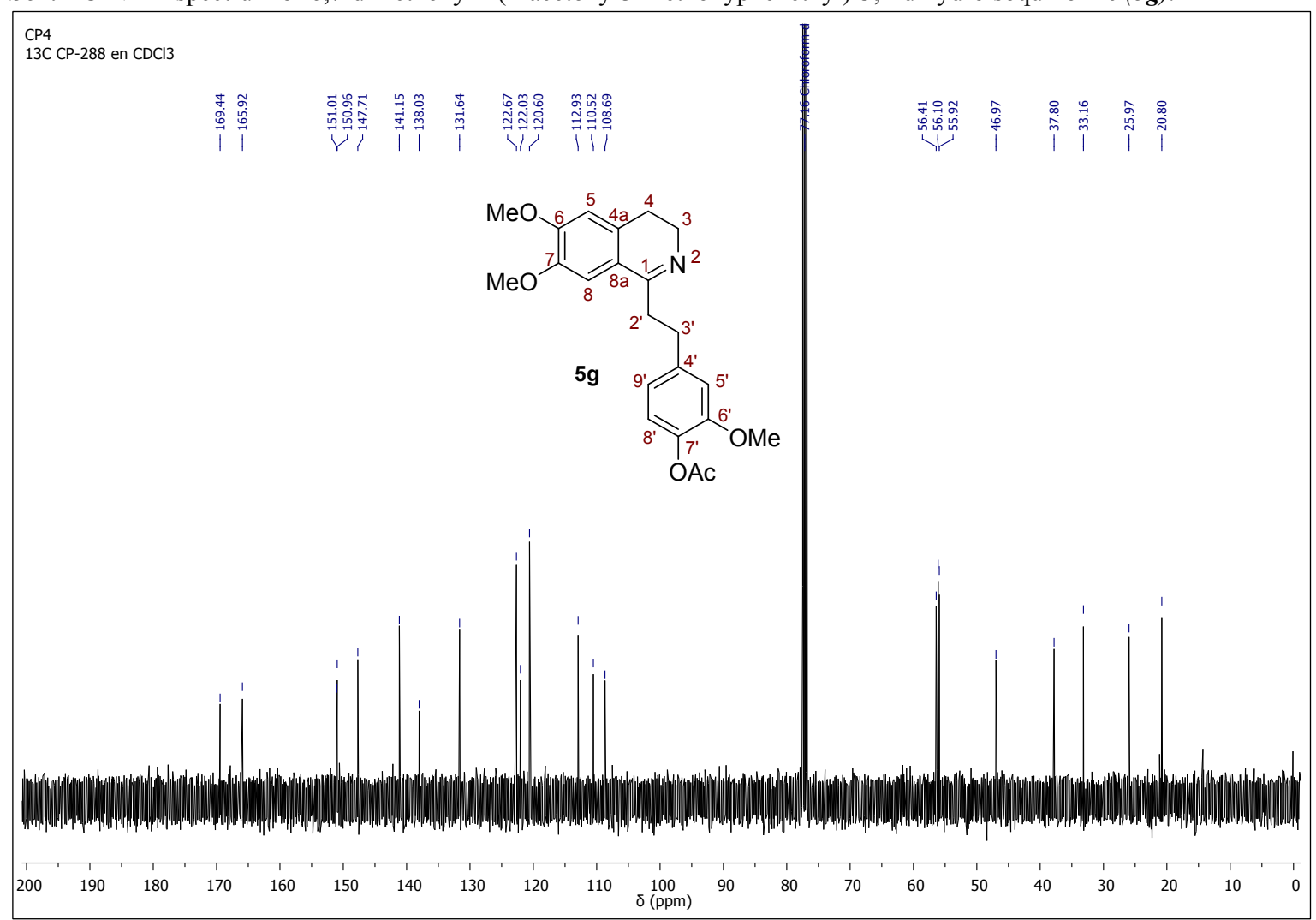


Figure S62. DEPT-135 spectrum of 6,7-dimethoxy-1-(4-acetoxy-3-methoxyphenethyl)-3,4-dihydroisoquinoline (5g).

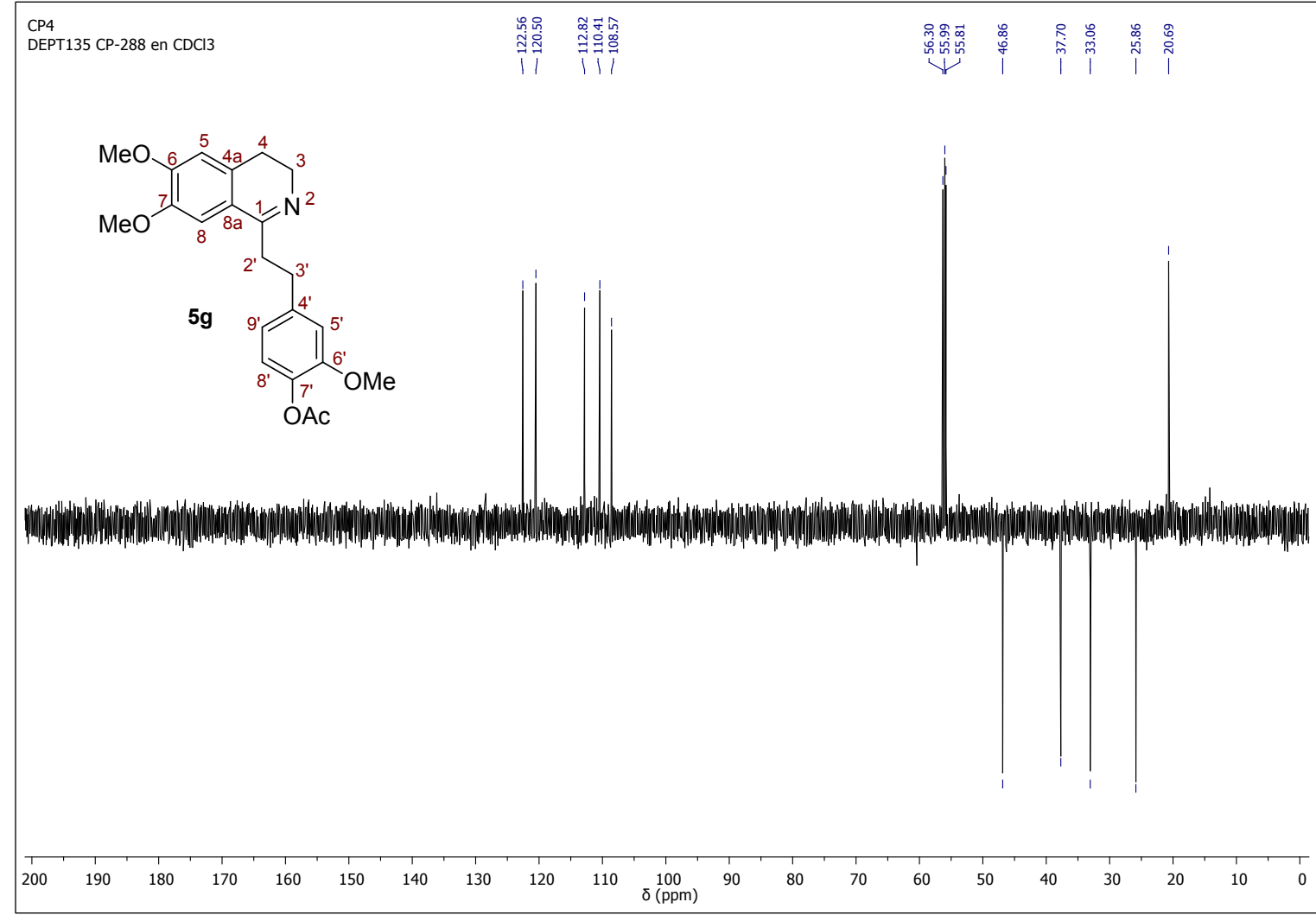

Figure S63. ${ }^{1}$ H-NMR spectrum of 6,7-dimethoxy-1-(3-acetoxy-4-methoxyphenethyl)-3,4-dihydroisoquinoline (5h).

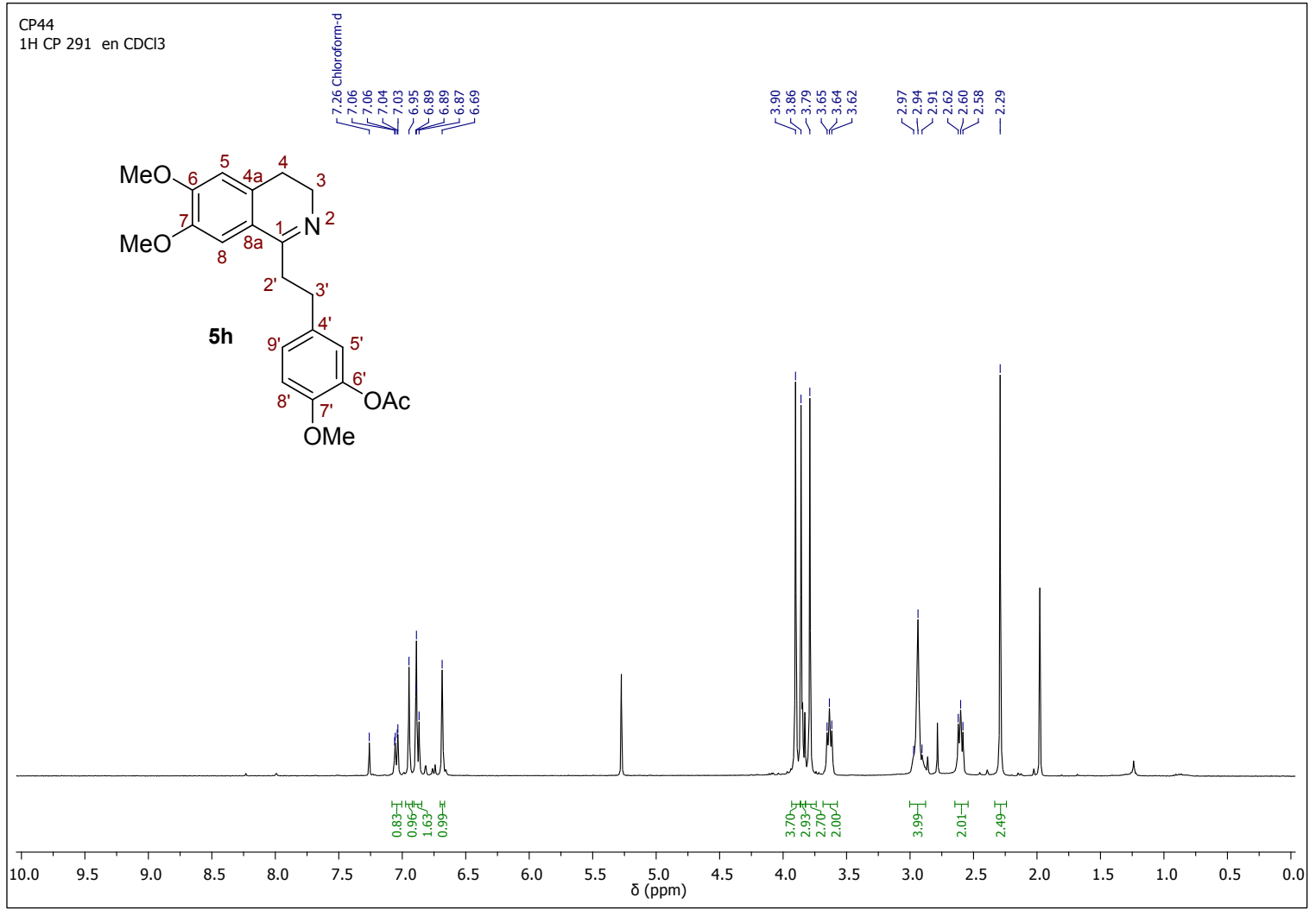


Figure S64. ${ }^{13}$ C-NMR spectrum of 6,7-dimethoxy-1-(3-acetoxy-4-methoxyphenethyl)-3,4-dihydroisoquinoline (5h).

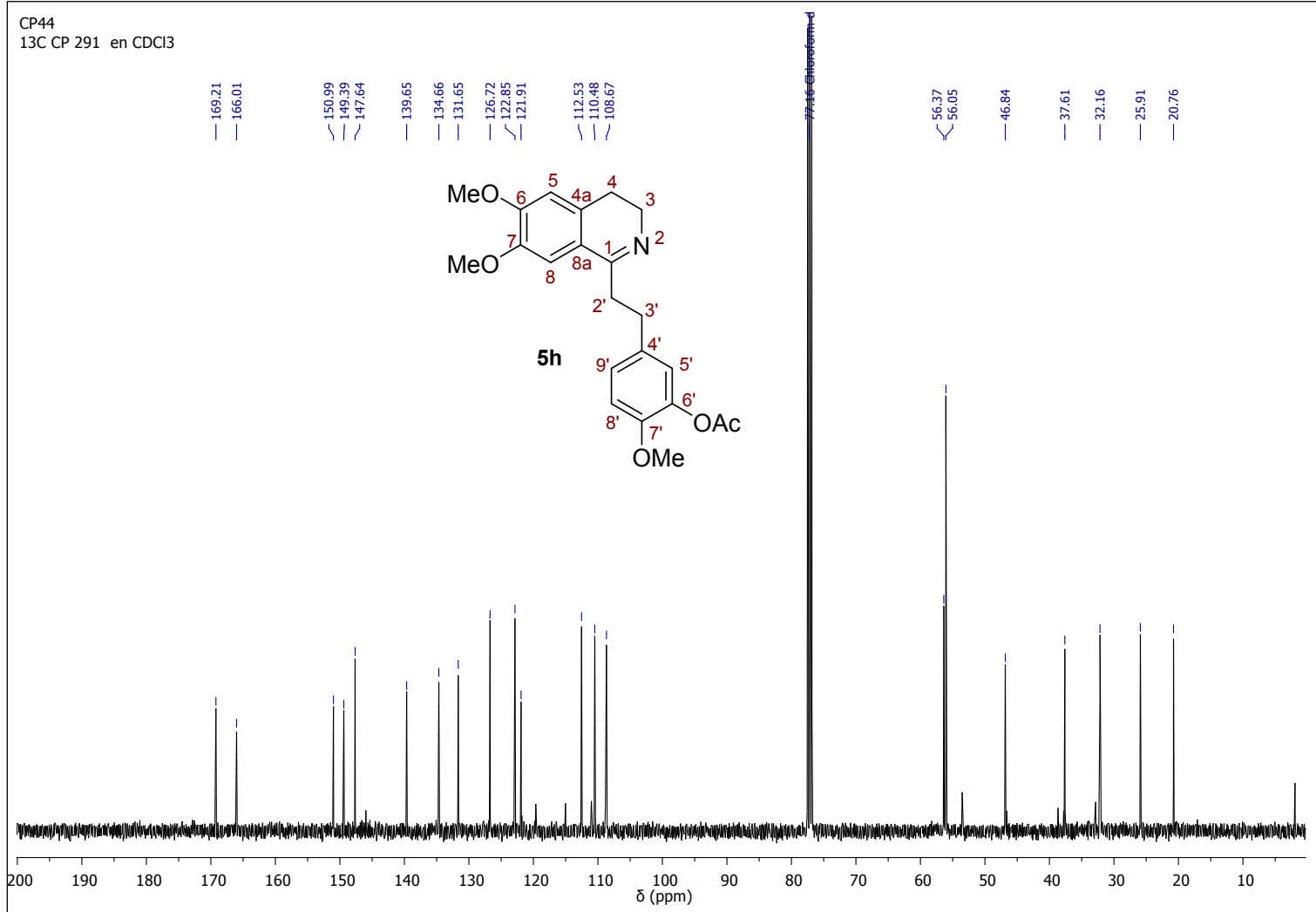

Figure S65. DEPT-135 spectrum of 6,7-dimethoxy-1-(3-acetoxy-4-methoxyphenethyl)-3,4-dihydroisoquinoline (5h).

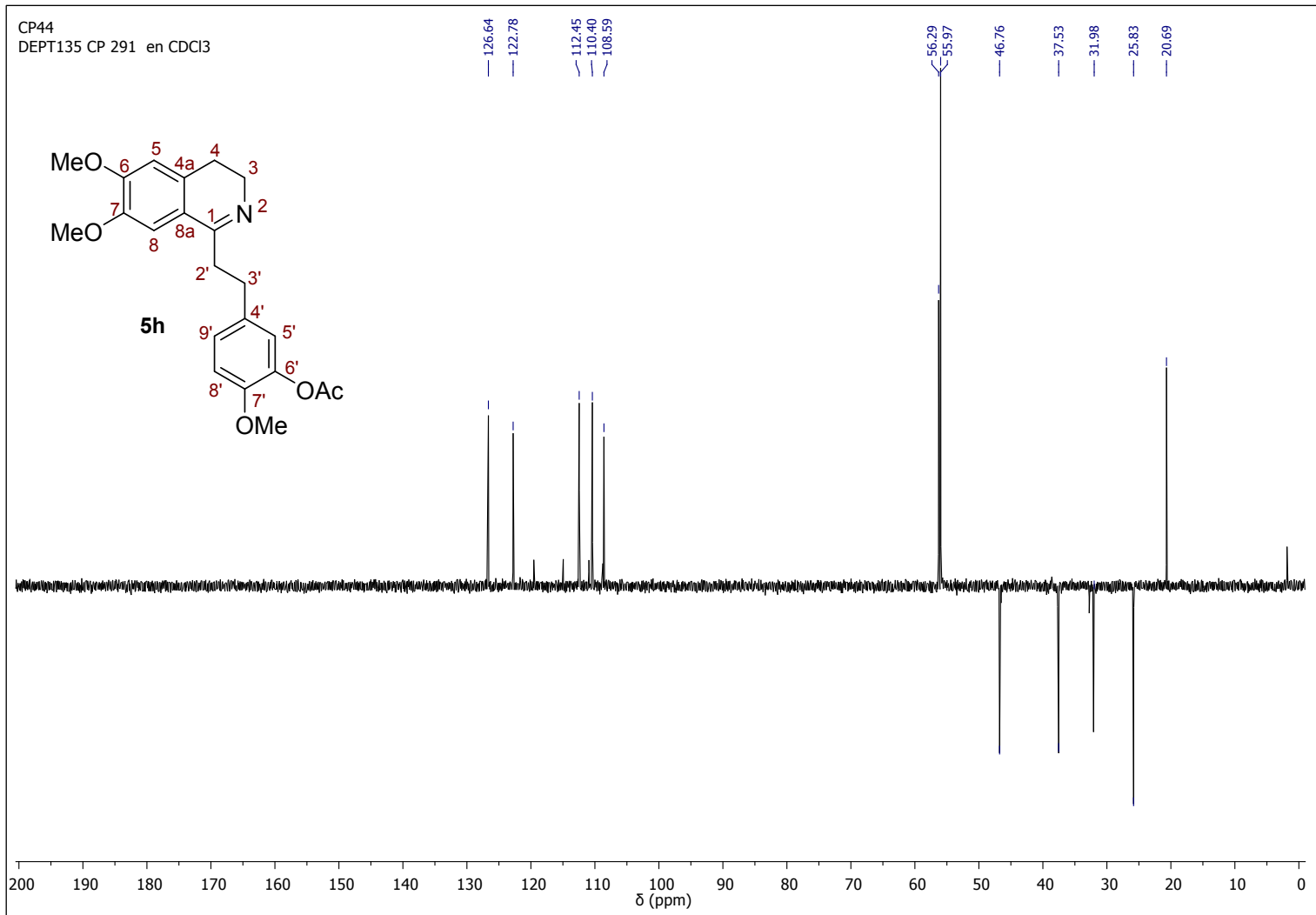


Figure S66. ${ }^{1}$ H-NMR spectrum of 6,7-dimethoxy-1-(3-acetoxy-3,4-dimethoxyphenethyl)-3,4-dihydroisoquinoline (5i).

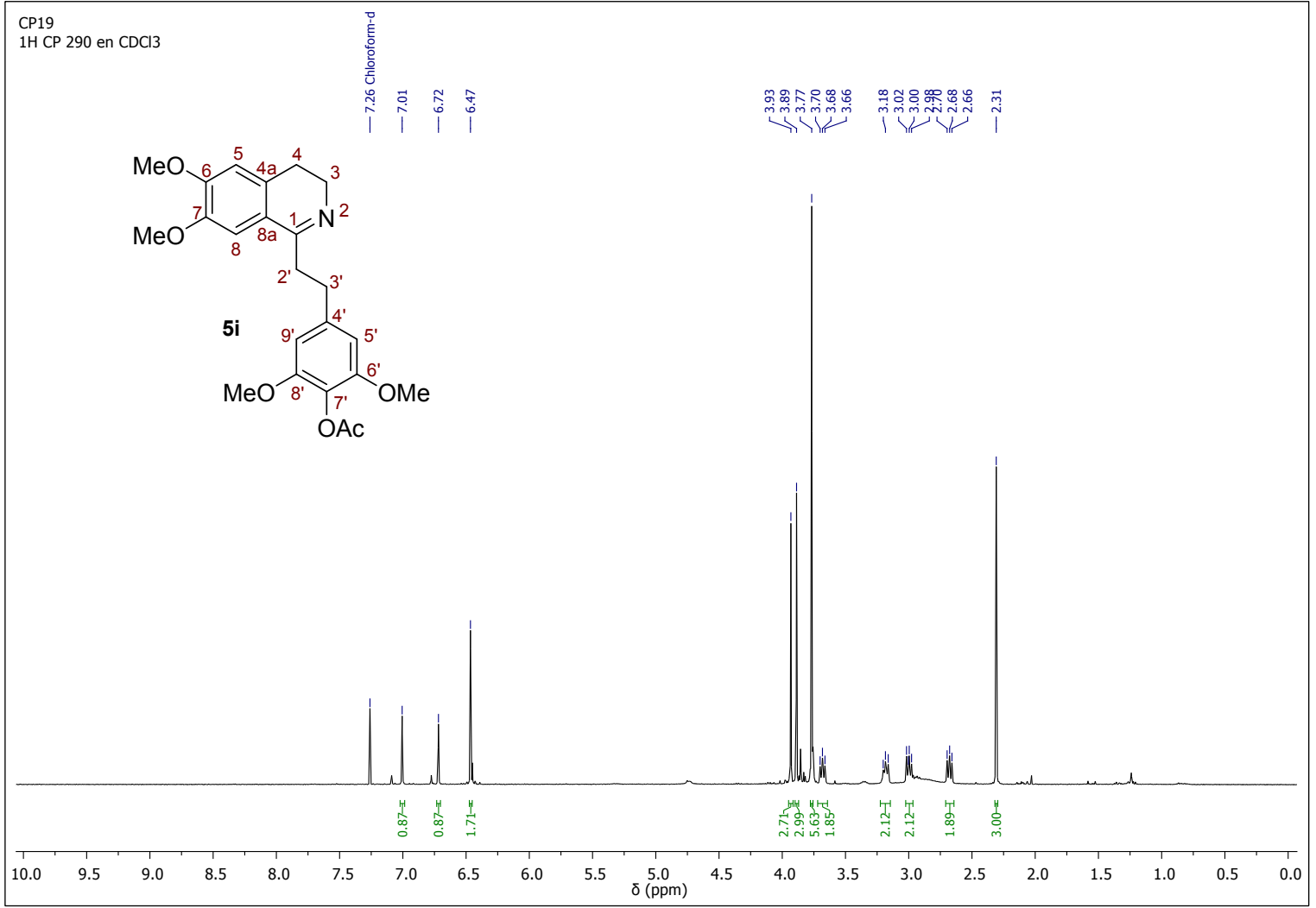

Figure S67. ${ }^{13} \mathrm{C}$-NMR spectrum of 6,7-dimethoxy-1-(3-acetoxy-3,4-dimethoxyphenethyl)-3,4-dihydroisoquinoline (5i).

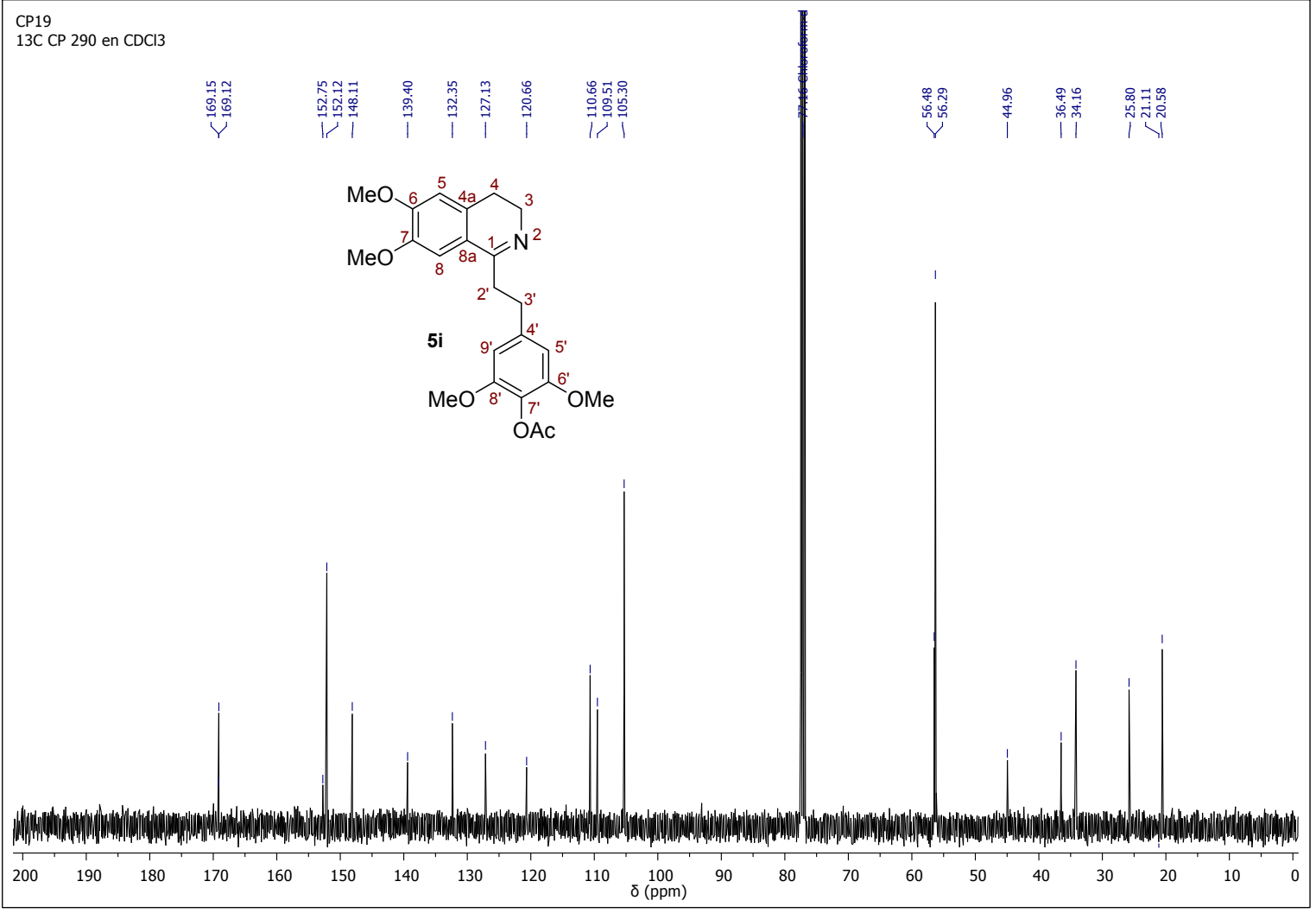


Figure S68. DEPT-135 spectrum of 6,7-dimethoxy-1-(3-acetoxy-3,4-dimethoxyphenethyl)-3,4-dihydroisoquinoline (5i).

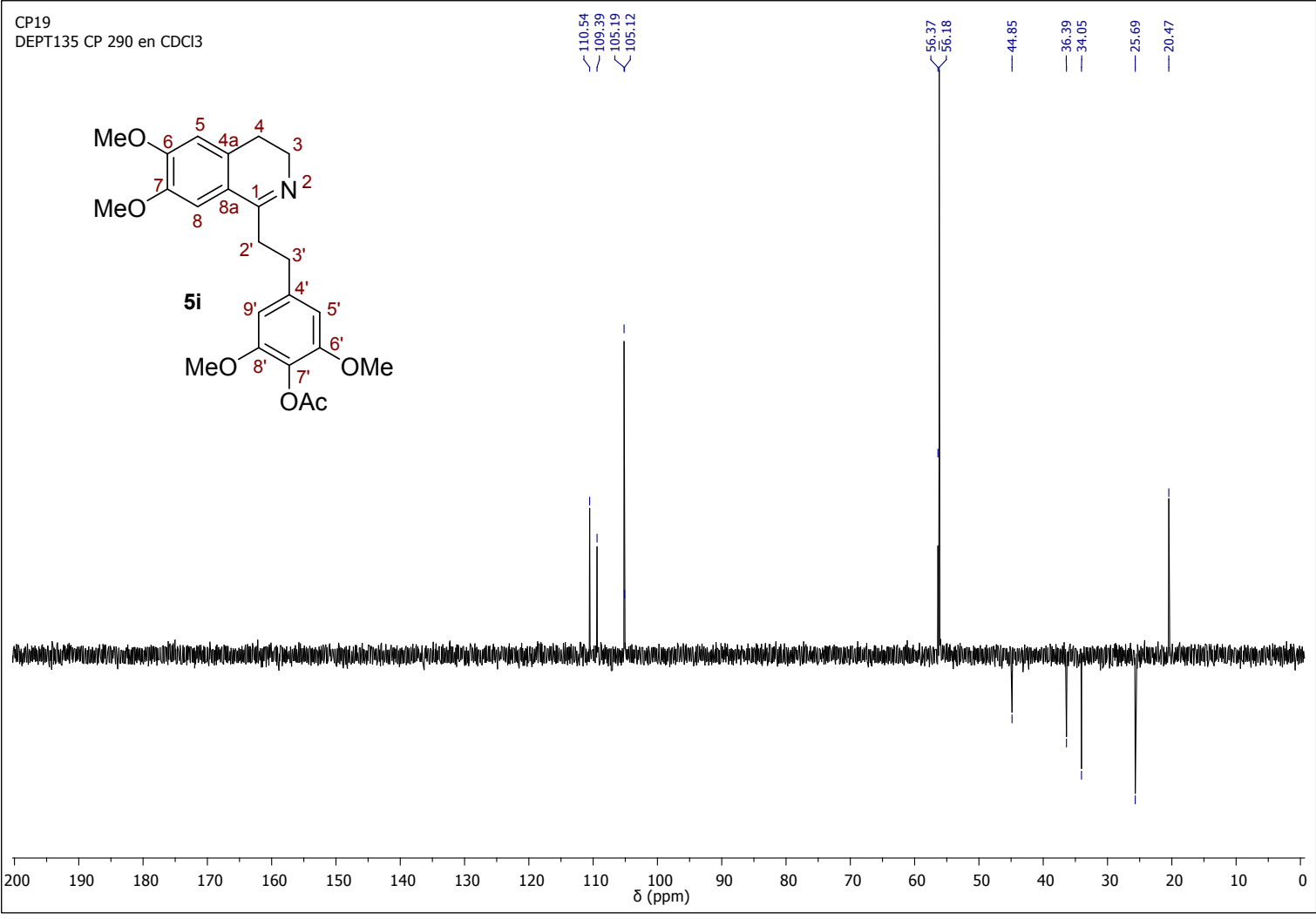




\section{Copies of ${ }^{1} \mathrm{H}$ NMR, ${ }^{13} \mathrm{C}$ NMR and DEPT-135 charts of $(R)-7$ a-i and $(S)$-7a-i Dysoxylum alkaloids}

Figure S69. ${ }^{1} \mathrm{H}$-NMR spectrum of $(R)$-6,7-dimethoxy- $N$-methyl-1-phenethyl-1,2,3,4-tetrahydroisoquinoline $(\boldsymbol{R})$-7 $\mathbf{a}$.

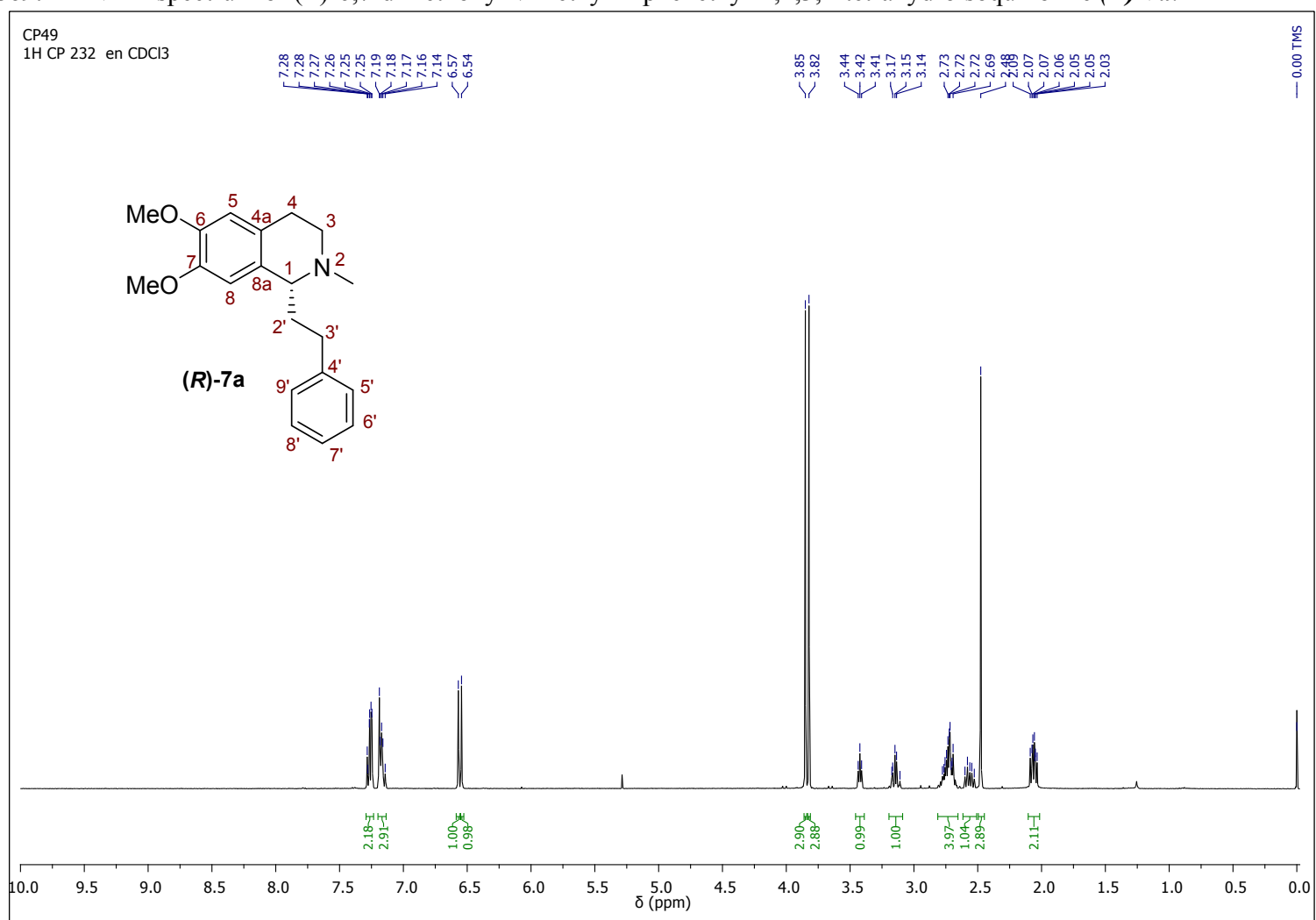

Figure S70. ${ }^{13} \mathrm{C}-\mathrm{NMR}$ spectrum of $(R)$-6,7-dimethoxy- $N$-methyl-1-phenethyl-1,2,3,4-tetrahydroisoquinoline $(\boldsymbol{R})$-7a

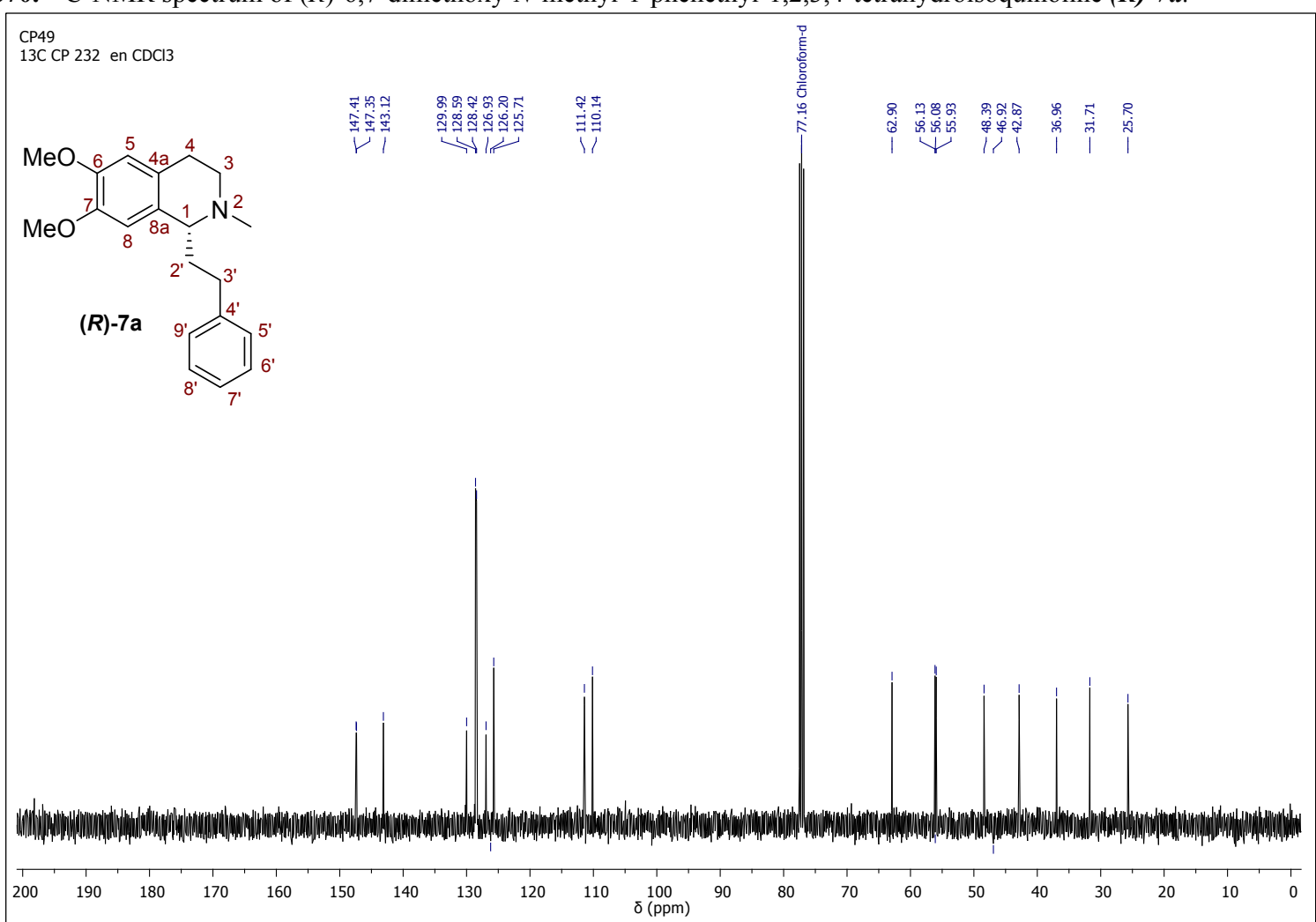


Figure S71. DEPT-135 spectrum of $(R)$-6,7-dimethoxy- $N$-methyl-1-phenethyl-1,2,3,4-tetrahydroisoquinoline (R)-7a.

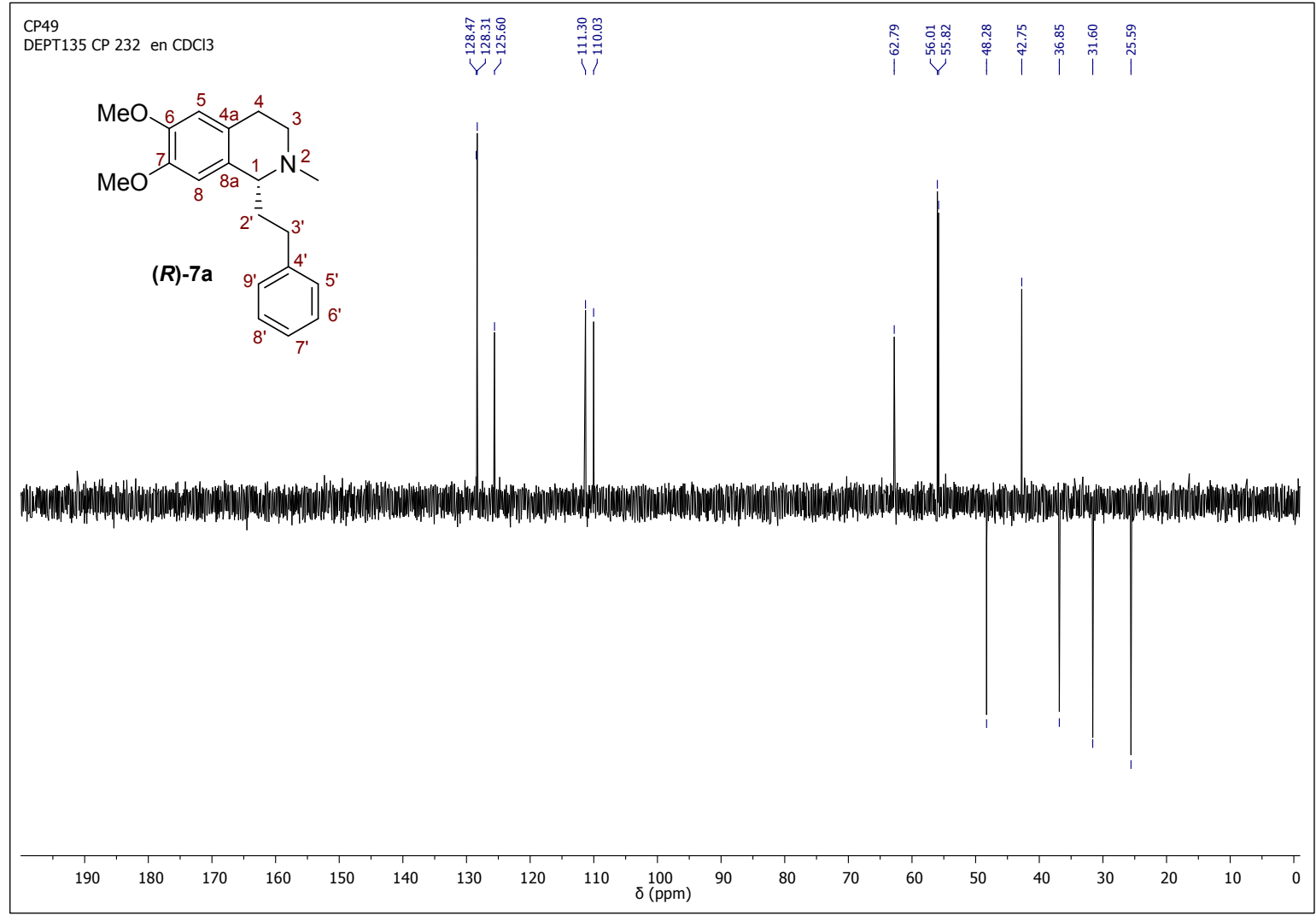

Figure S72. ${ }^{1} \mathrm{H}-\mathrm{NMR}$ spectrum of $(R)$-6,7-dimethoxy-1-(4-methoxyphenethyl)- $N$-methyl-1,2,3,4-tetrahydroisoquinoline (R)-7b.

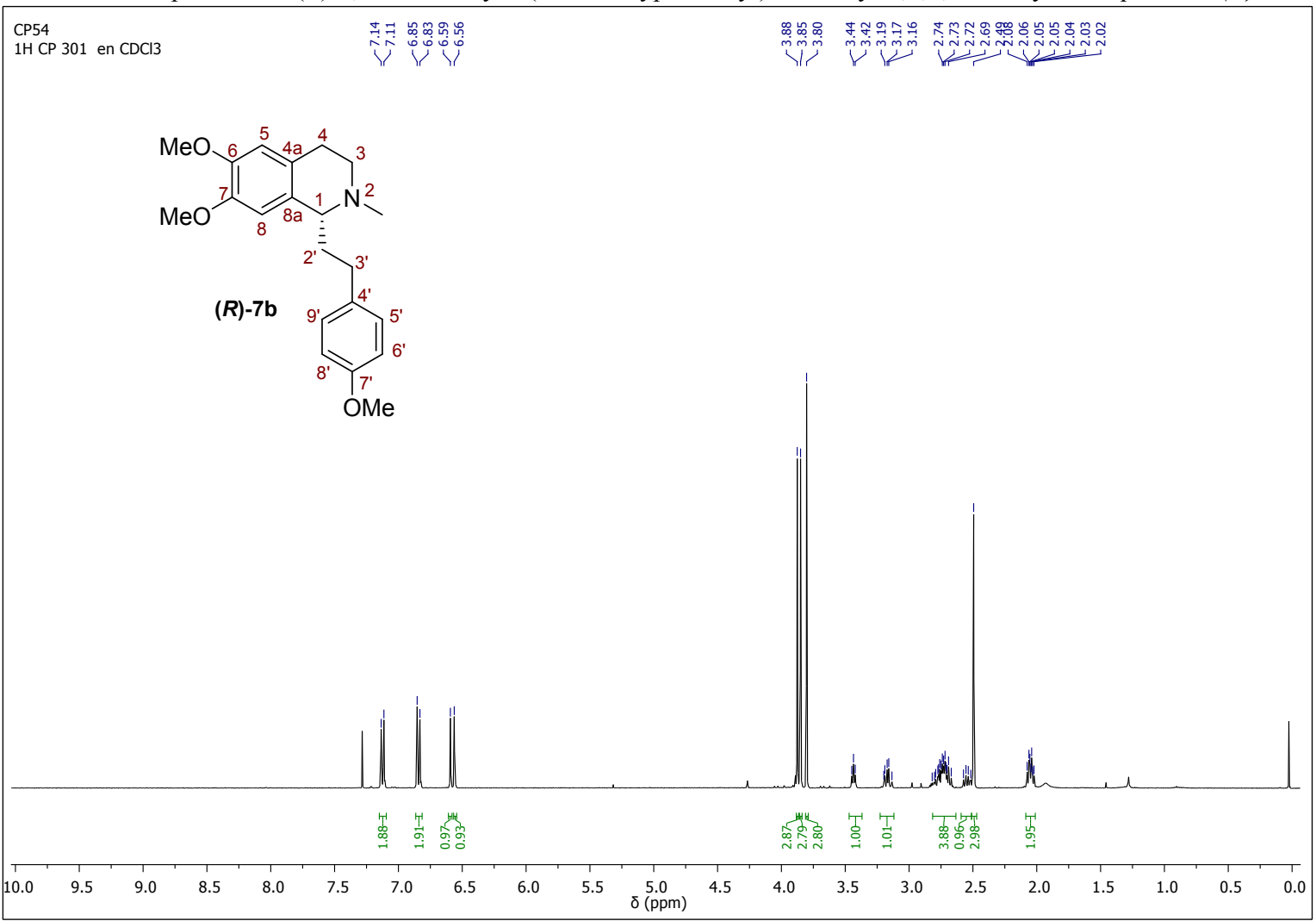


Figure S73. ${ }^{13} \mathrm{C}$-NMR spectrum of (R)-6,7-dimethoxy-1-(4-methoxyphenethyl)- $N$-methyl-1,2,3,4-tetrahydroisoquinoline (R)-7b

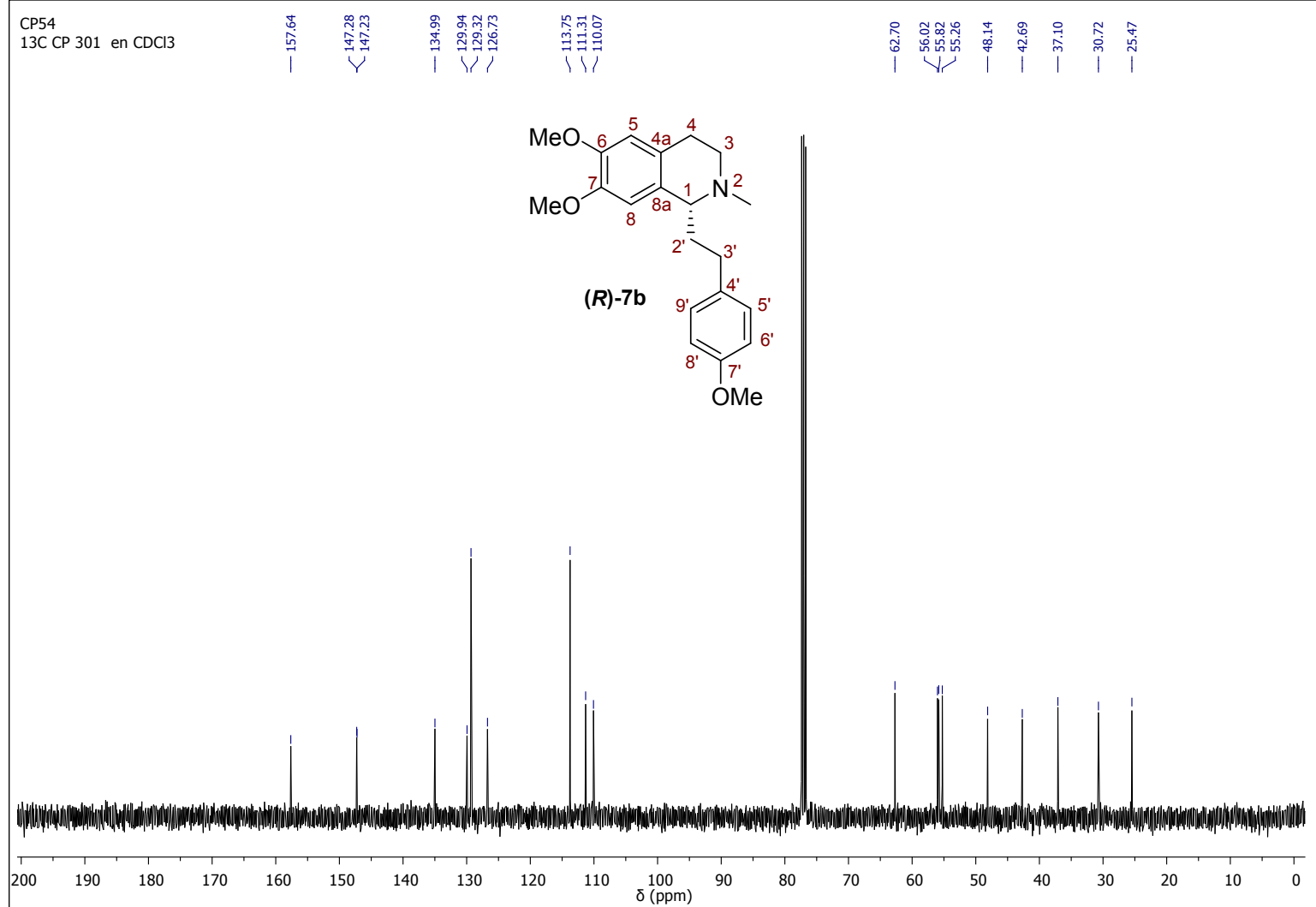

Figure S74. DEPT-135 spectrum of ( $R$ )-6,7-dimethoxy-1-(4-methoxyphenethyl)- $N$-methyl-1,2,3,4-tetrahydroisoquinoline (R)-7b .

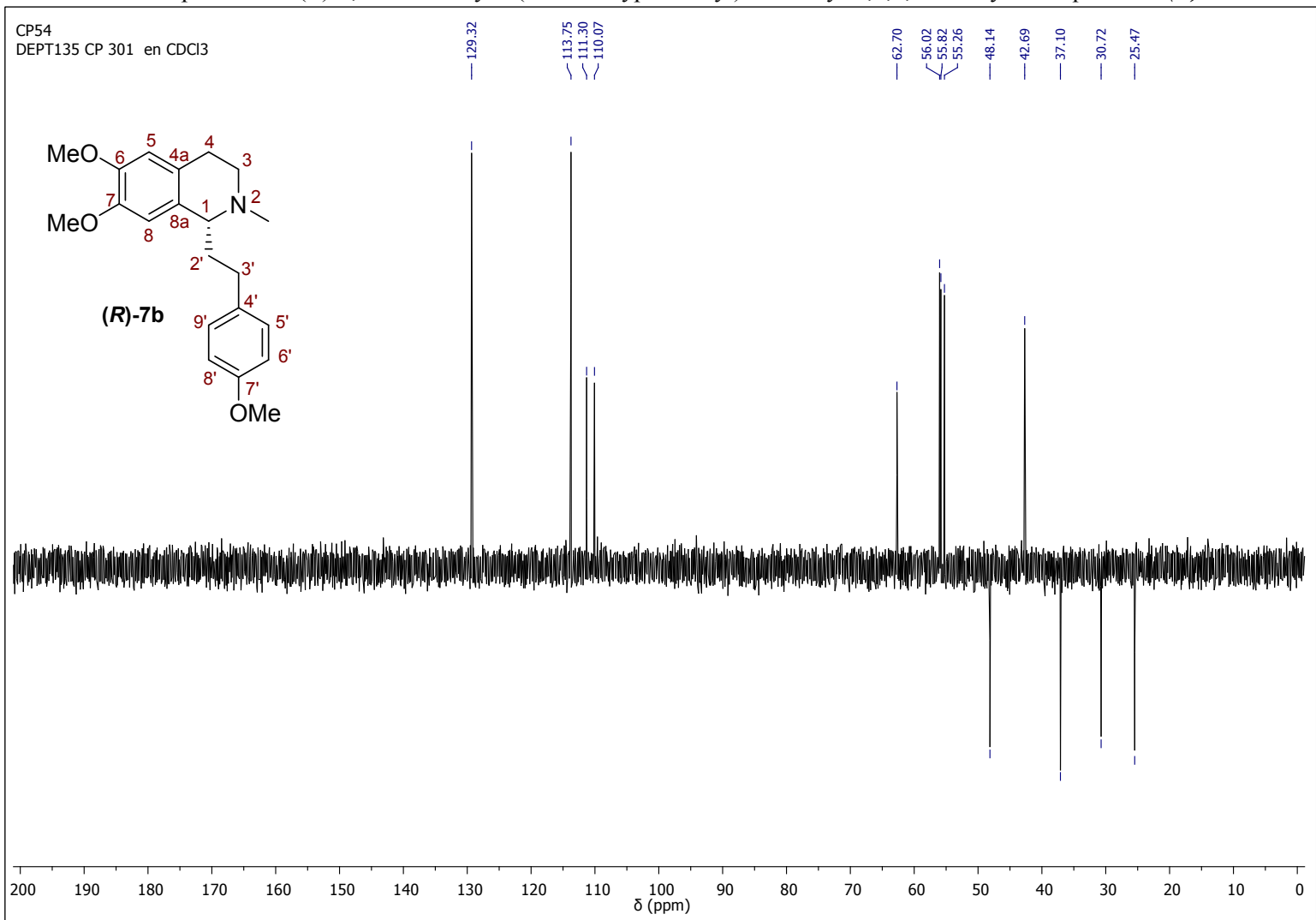


Figure S75. ${ }^{1} \mathrm{H}-\mathrm{NMR}$ spectrum of $(R)-6,7-\operatorname{dimethoxy}-1-(3,4-\operatorname{dimethoxyphenethyl)-~} N$-methyl-1,2,3,4-tetrahydroisoquinoline $(\boldsymbol{R})$ - $7 \boldsymbol{c}$.

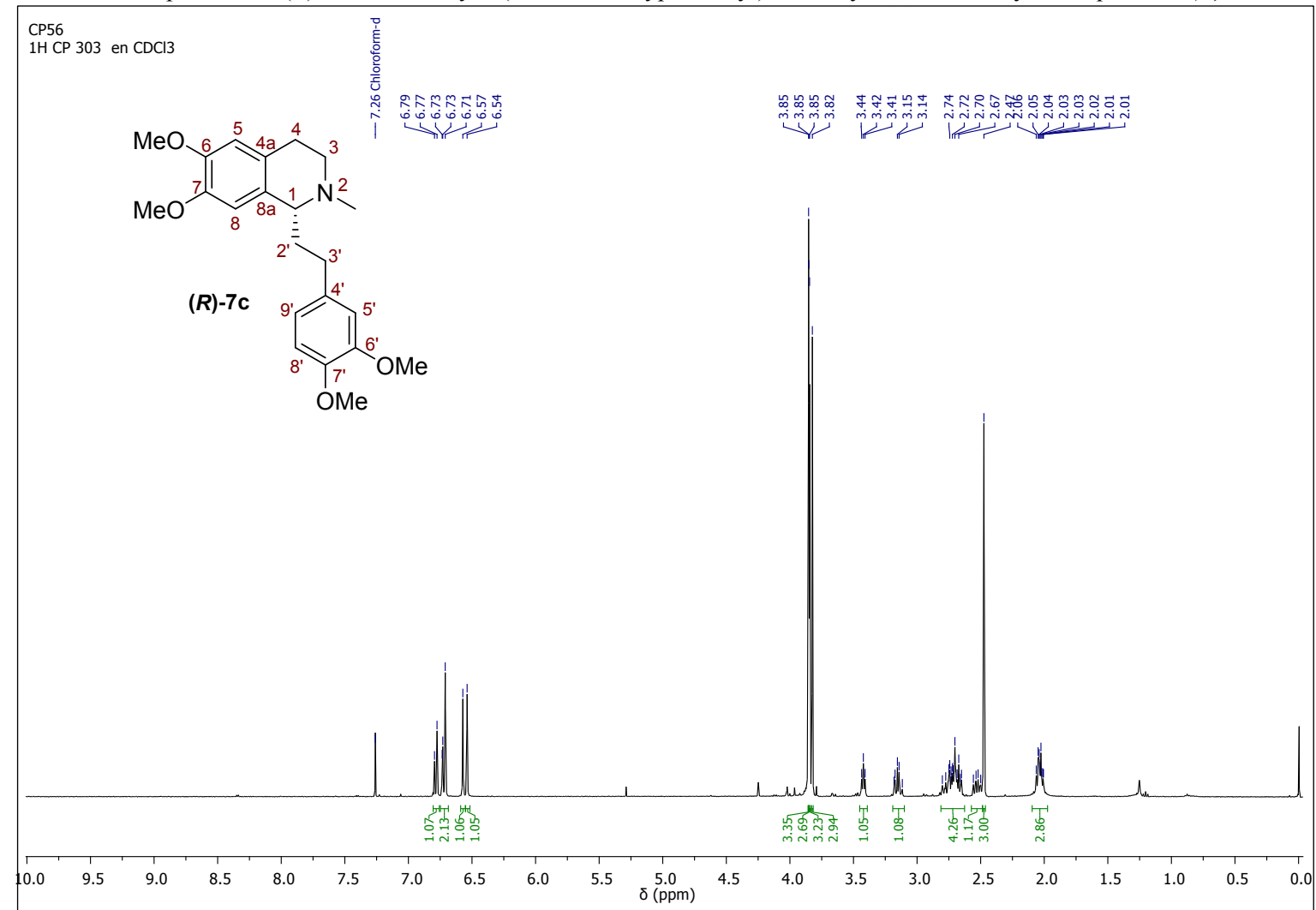

Figure S76. ${ }^{13} \mathrm{C}$-NMR spectrum of $(R)$-6,7-dimethoxy-1-(3,4-dimethoxyphenethyl)- $N$-methyl-1,2,3,4-tetrahydroisoquinoline (R)-7c

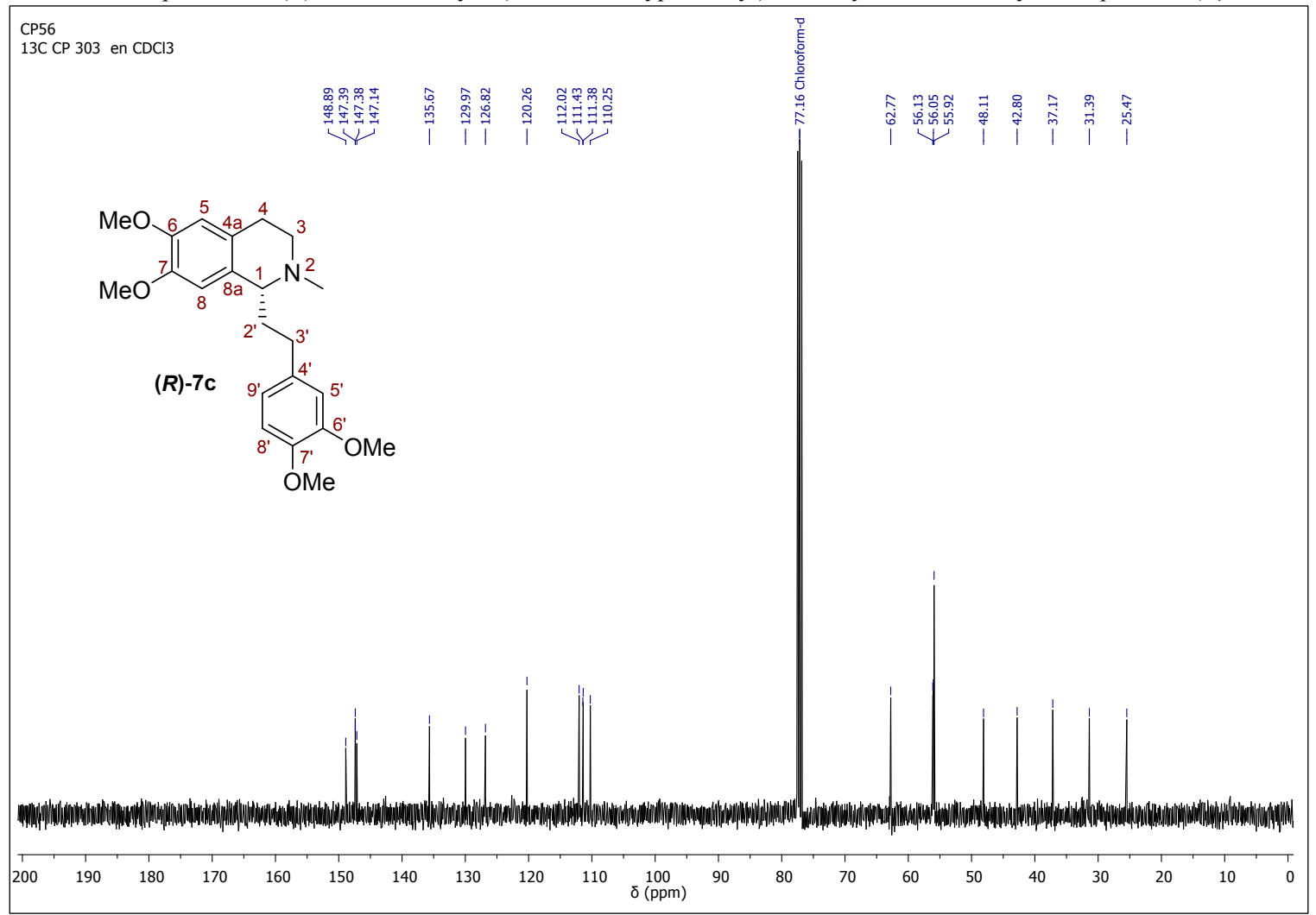

Figure S77. DEPT-135 spectrum of ( $R$ )-6,7-dimethoxy-1-(3,4-dimethoxyphenethyl)- $N$-methyl-1,2,3,4-tetrahydroisoquinoline (R)-7c. 


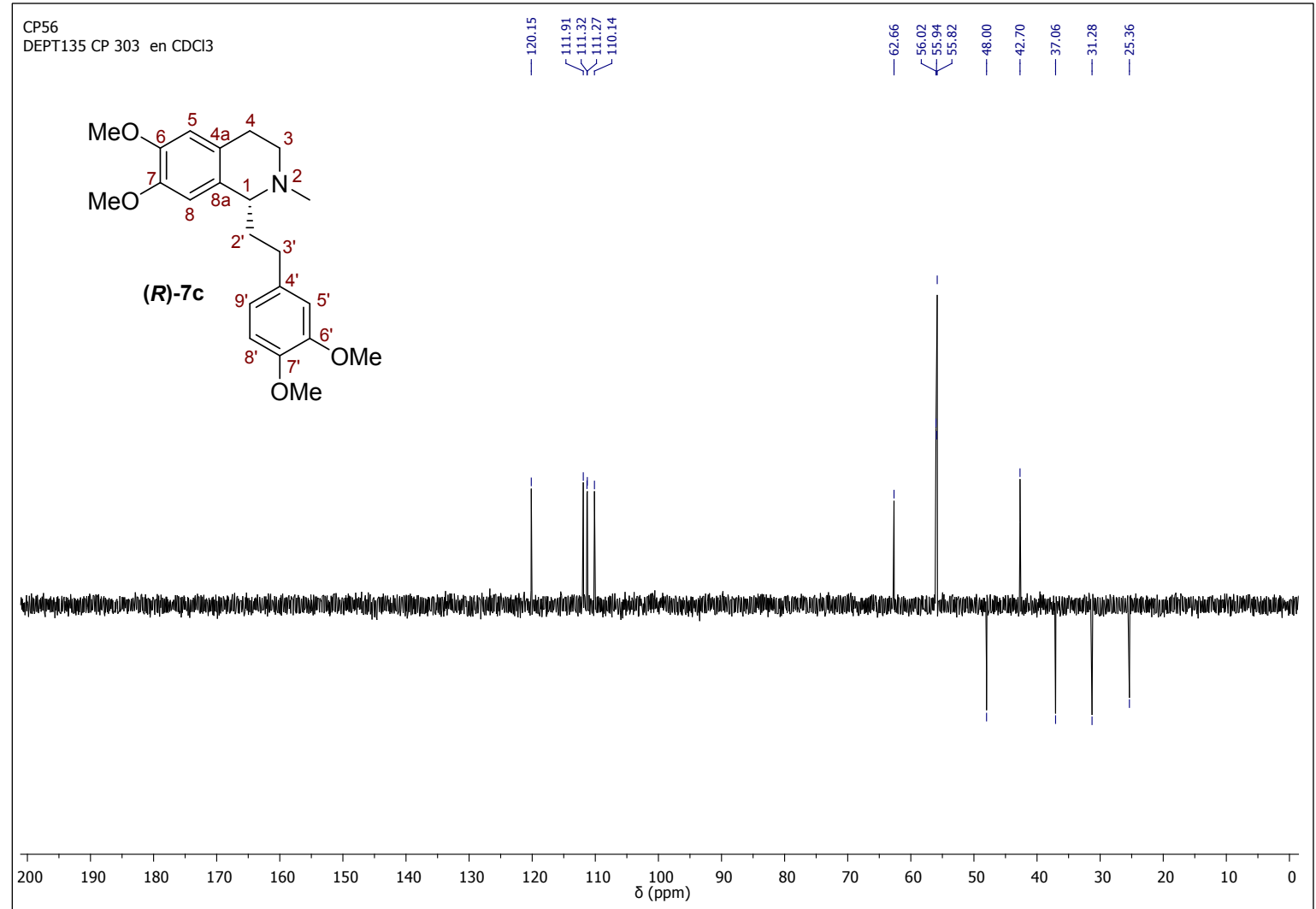

Figure S78. ${ }^{1} \mathrm{H}$-NMR spectrum of $(R)$-6,7-dimethoxy-1-(3,4,5-trimethoxyphenethyl)- $N$-methyl-1,2,3,4-tetrahydroisoquinoline (R)-7d.

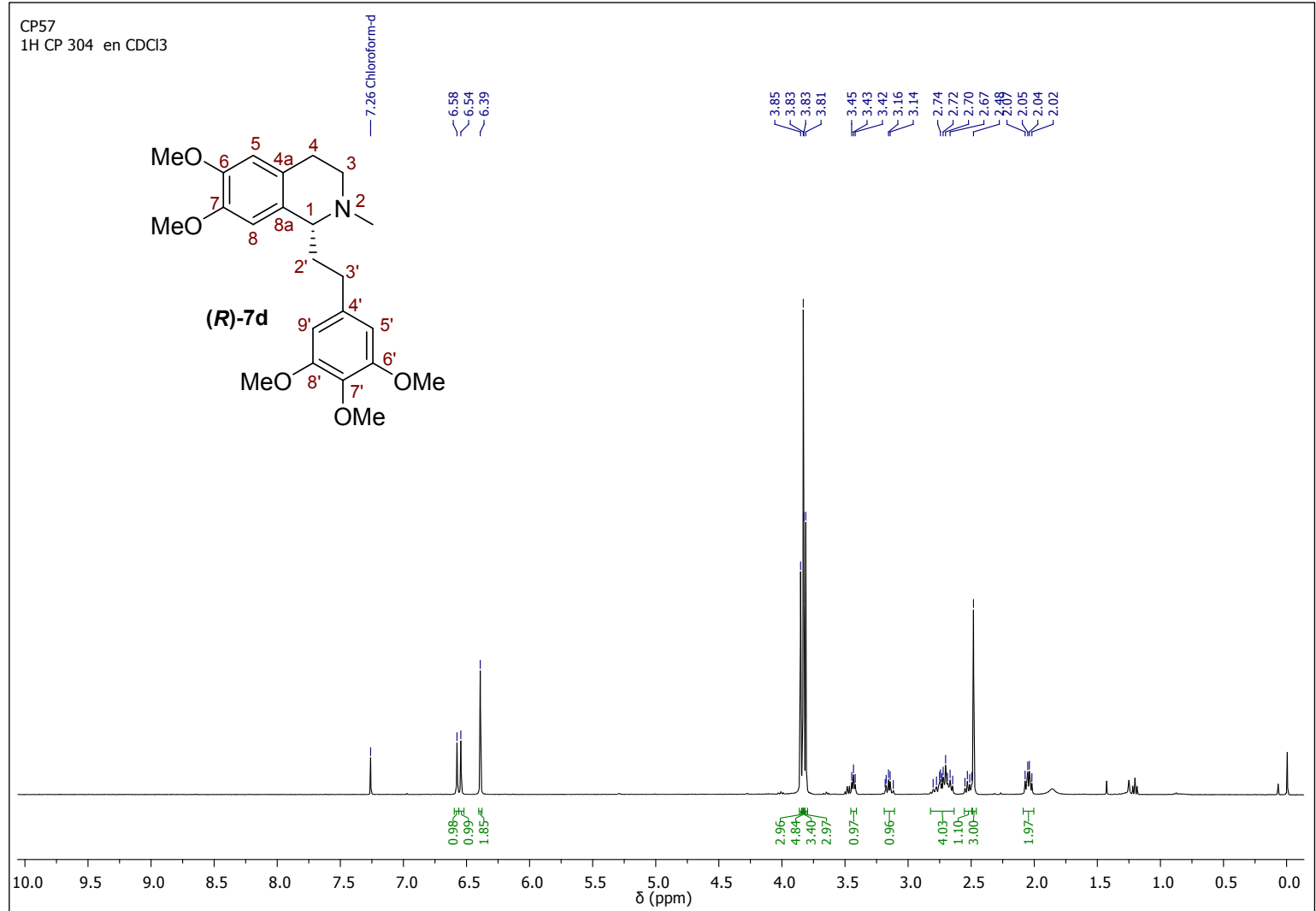

Figure S79. ${ }^{13} \mathrm{C}$-NMR spectrum of $(R)-6,7$-dimethoxy-1-(3,4,5-trimethoxyphenethyl)- $N$-methyl-1,2,3,4-tetrahydroisoquinoline (R)-7d 


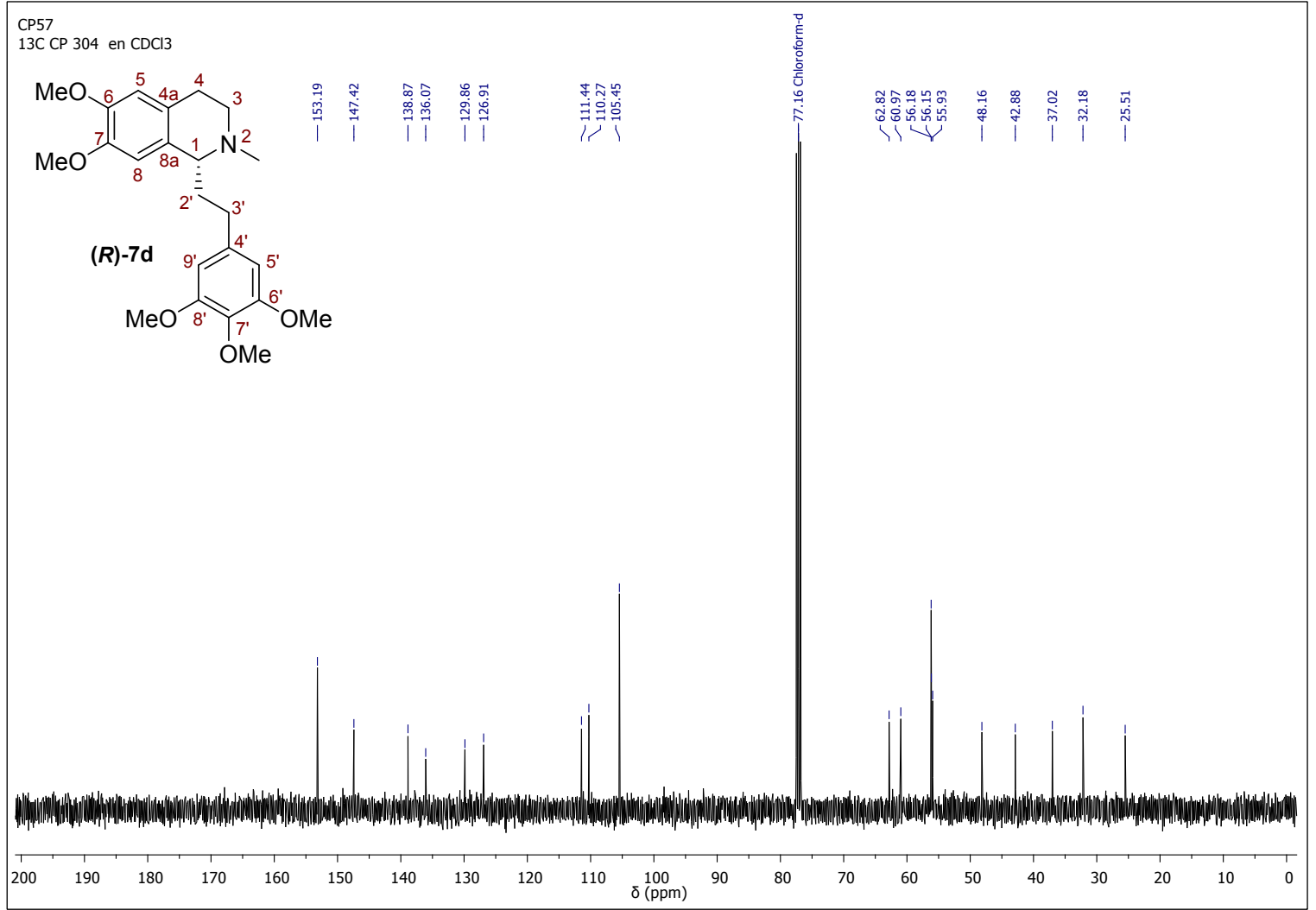

Figure S80. DEPT-135 spectrum of (R)-6,7-dimethoxy-1-(3,4,5-trimethoxyphenethyl)- $N$-methyl-1,2,3,4-tetrahydroisoquinoline (R)-7d .

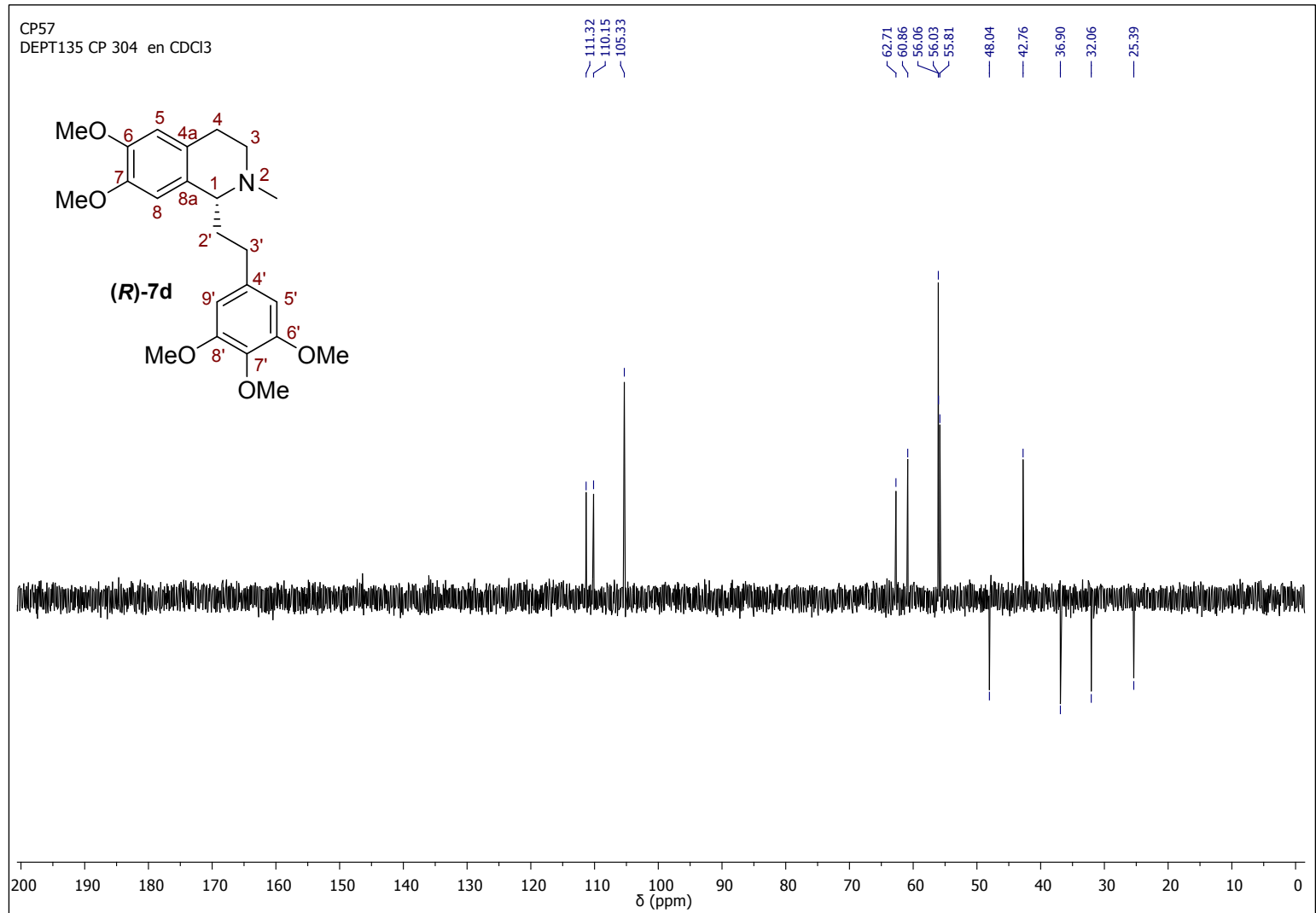

Figure S81. ${ }^{1} \mathrm{H}$-NMR spectrum of $(R)-6,7$-dimethoxy-1-(3,4-methylendioxyphenethyl)- $N$-methyl-1,2,3,4-tetrahydroisoquinoline (R)-7e . 


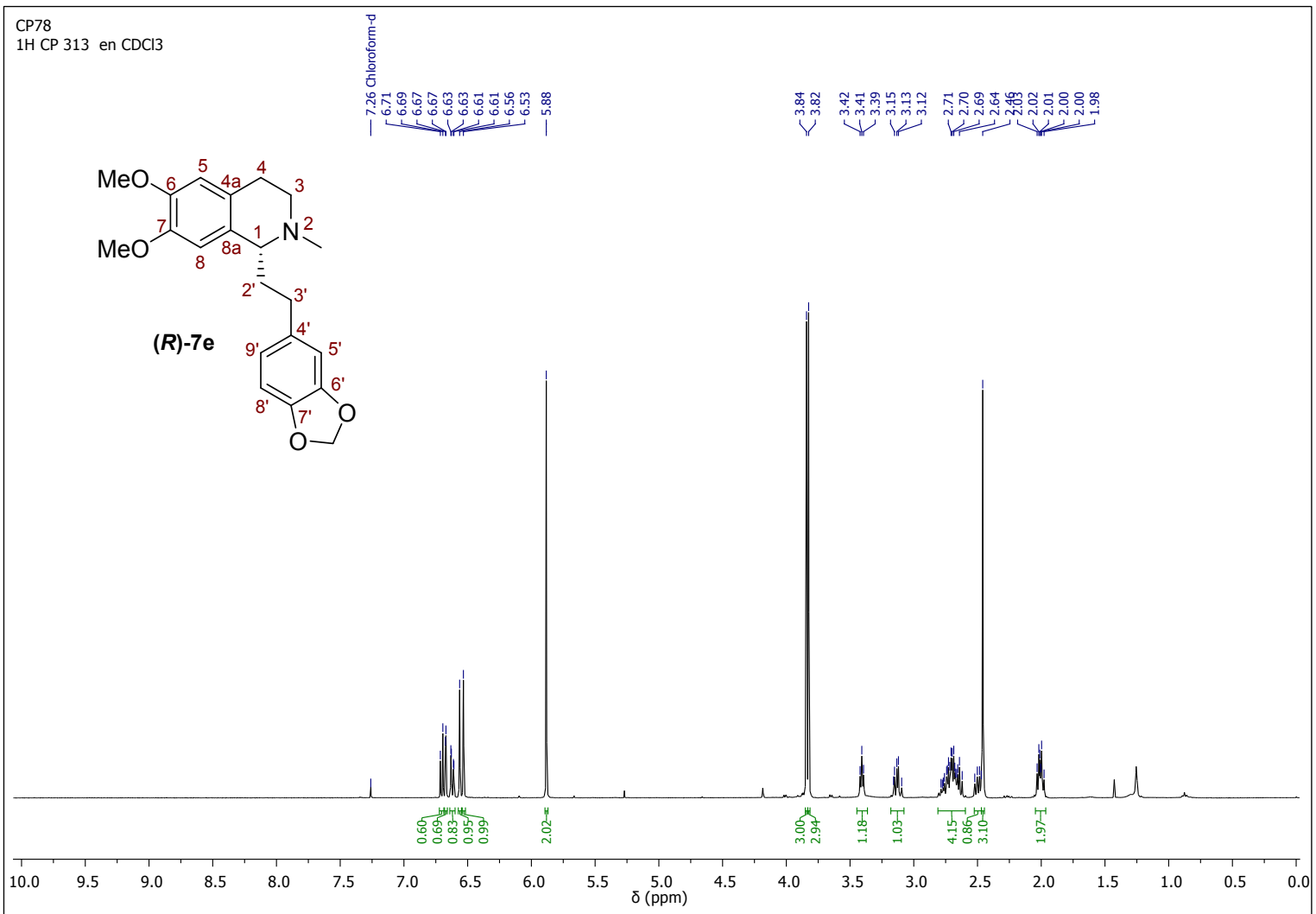

Figure S82. ${ }^{13} \mathrm{C}$-NMR spectrum of (R)-6,7-dimethoxy-1-(3,4-methylendioxyphenethyl)- $N$-methyl-1,2,3,4-tetrahydroisoquinoline (R)-7e .

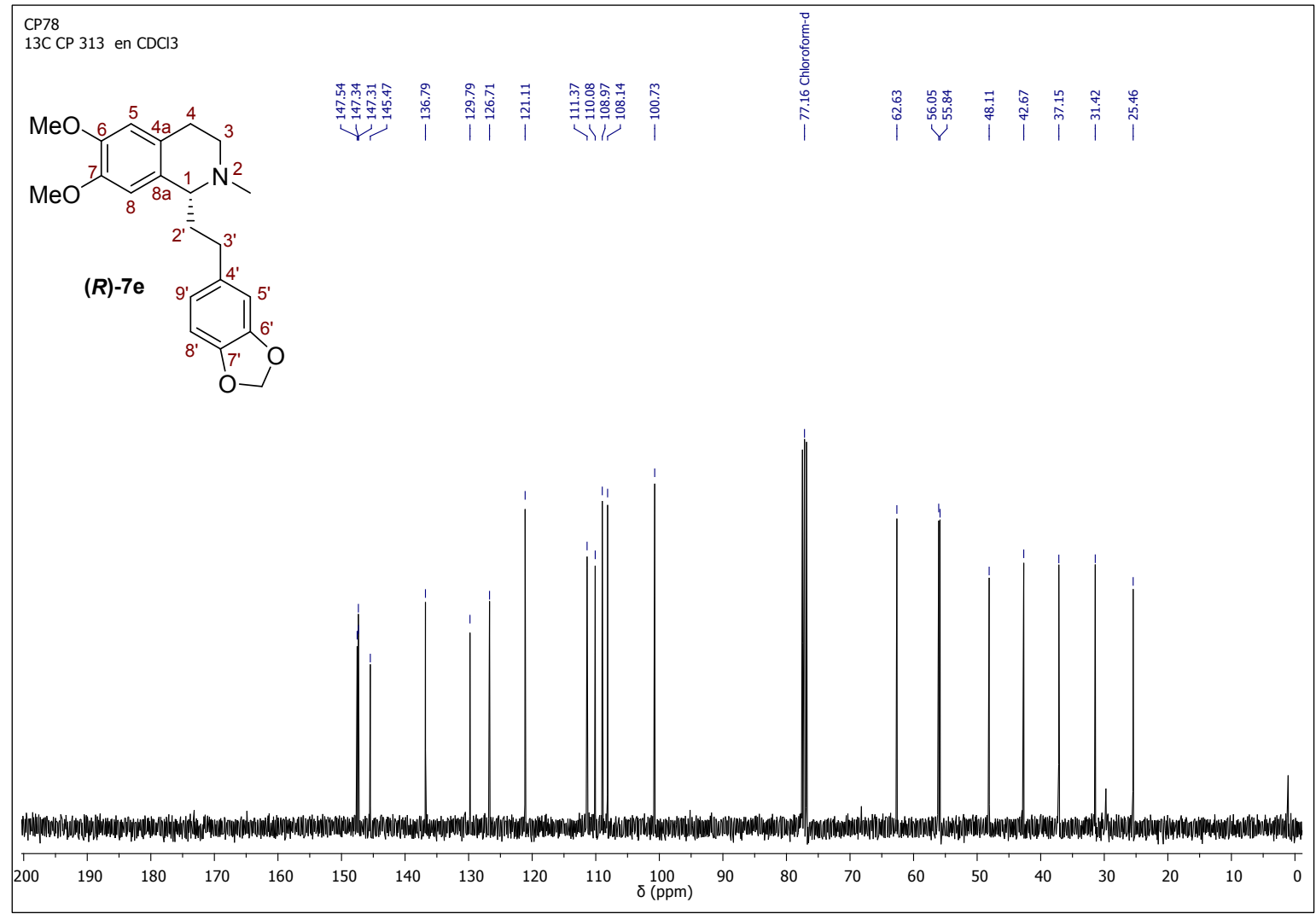

Figure S83. DEPT-135 spectrum of (R)-6,7-dimethoxy-1-(3,4-methylendioxyphenethyl)- $N$-methyl-1,2,3,4-tetrahydroisoquinoline (R)-7e 


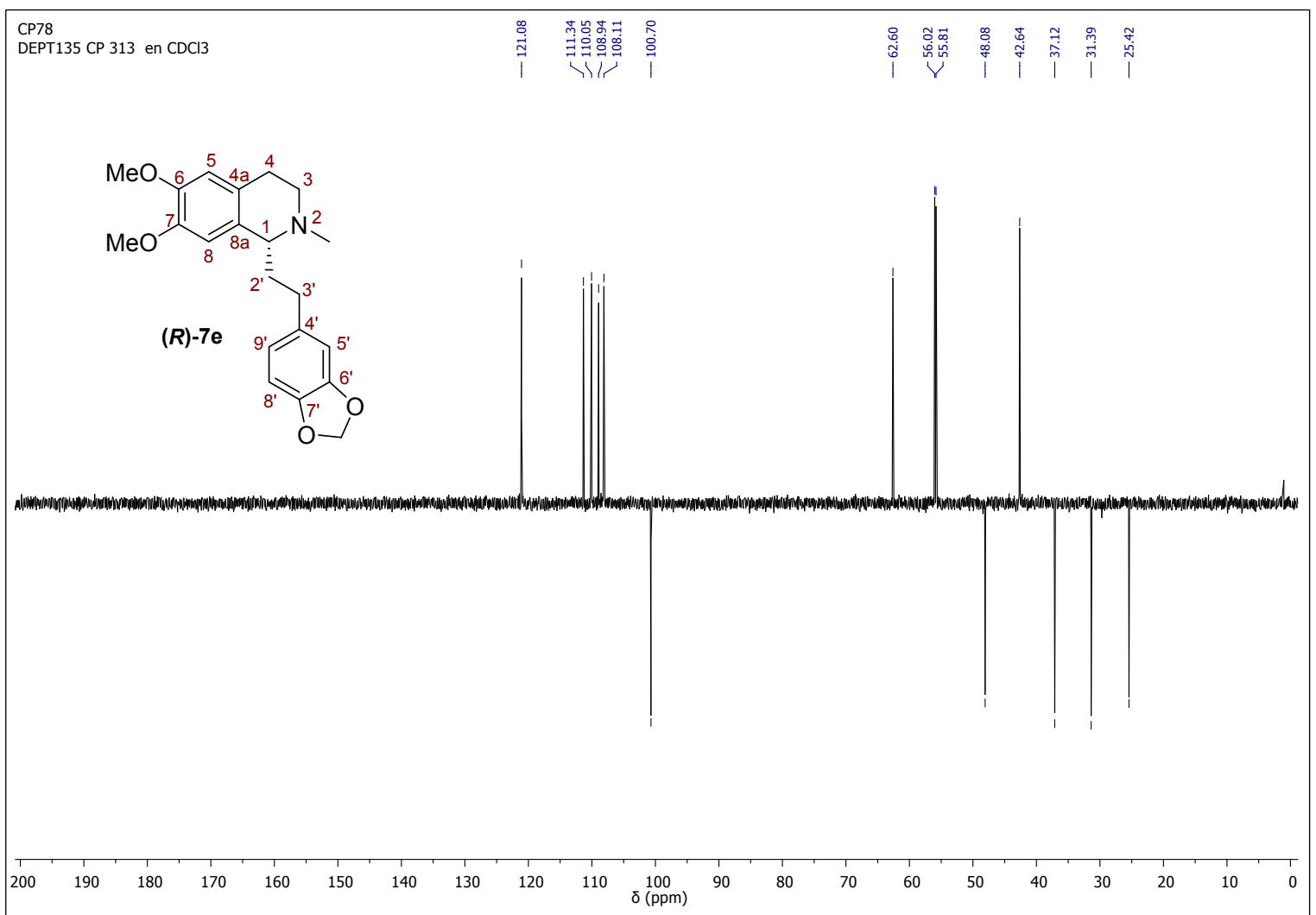

Figure S84. ${ }^{1} \mathrm{H}-\mathrm{NMR}$ spectrum of $(R)$-6,7-dimethoxy-1-(4-hydroxyphenethyl)- $N$-methyl-1,2,3,4-tetrahydroisoquinoline $(\boldsymbol{R})-7 \boldsymbol{f}$.

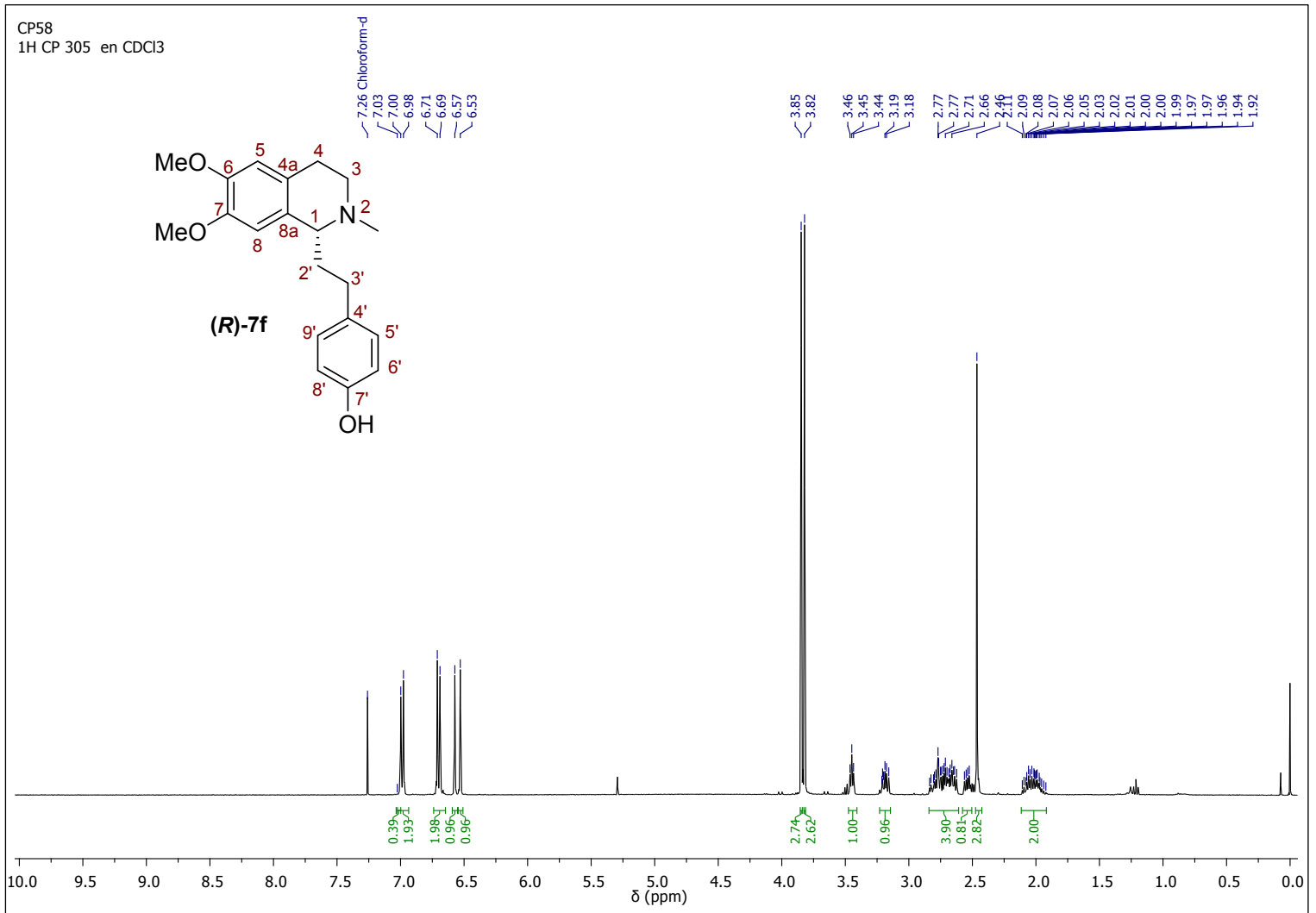

Figure S85. ${ }^{13} \mathrm{C}$-NMR spectrum of $(R)$-6,7-dimethoxy-1-(4-hydroxyphenethyl)- $N$-methyl-1,2,3,4-tetrahydroisoquinoline $(\boldsymbol{R})-7 \boldsymbol{f}$. 


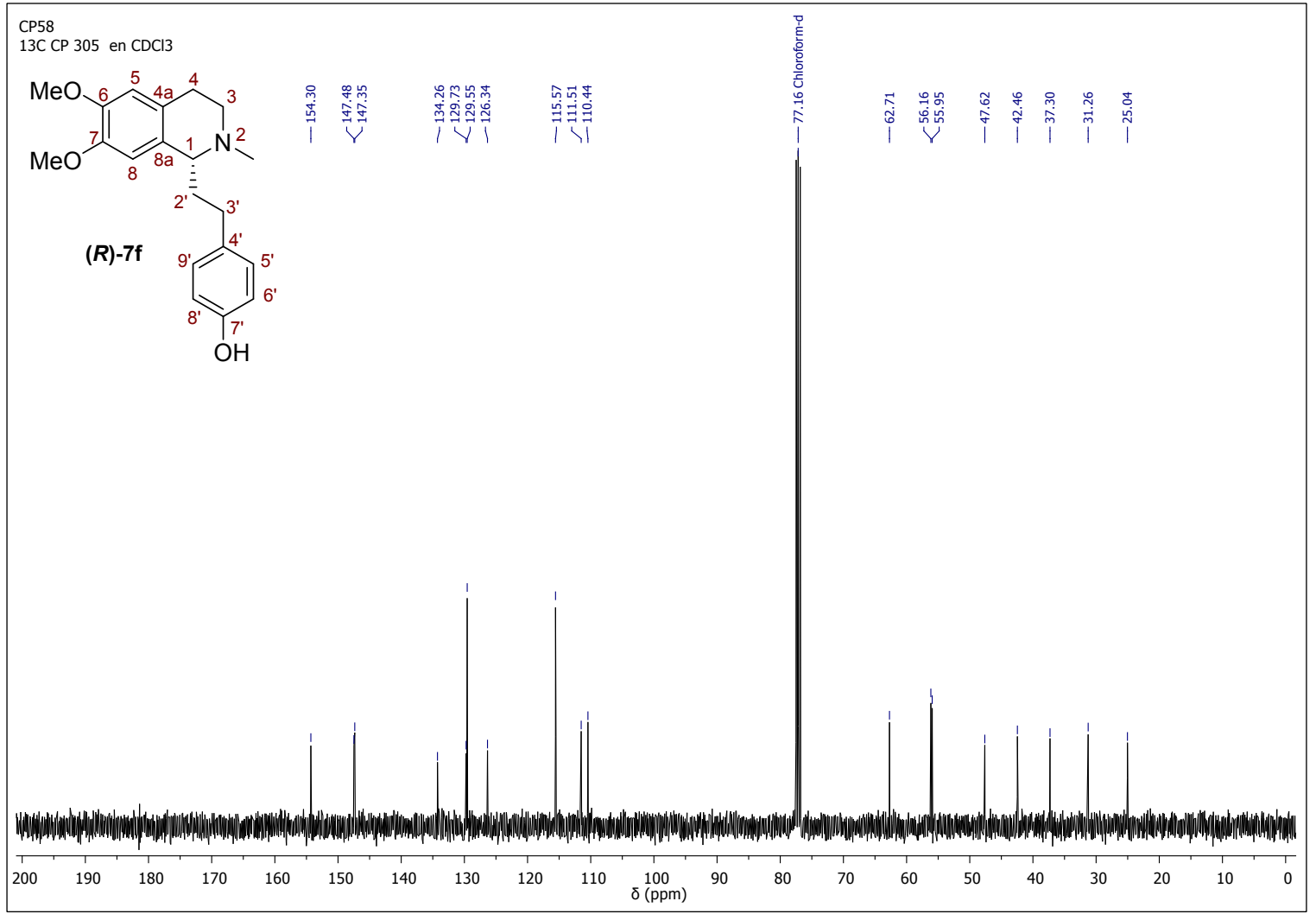

Figure S86. DEPT-135 spectrum of (R)-6,7-dimethoxy-1-(4-hydroxyphenethyl)- $N$-methyl-1,2,3,4-tetrahydroisoquinoline (R)-7f

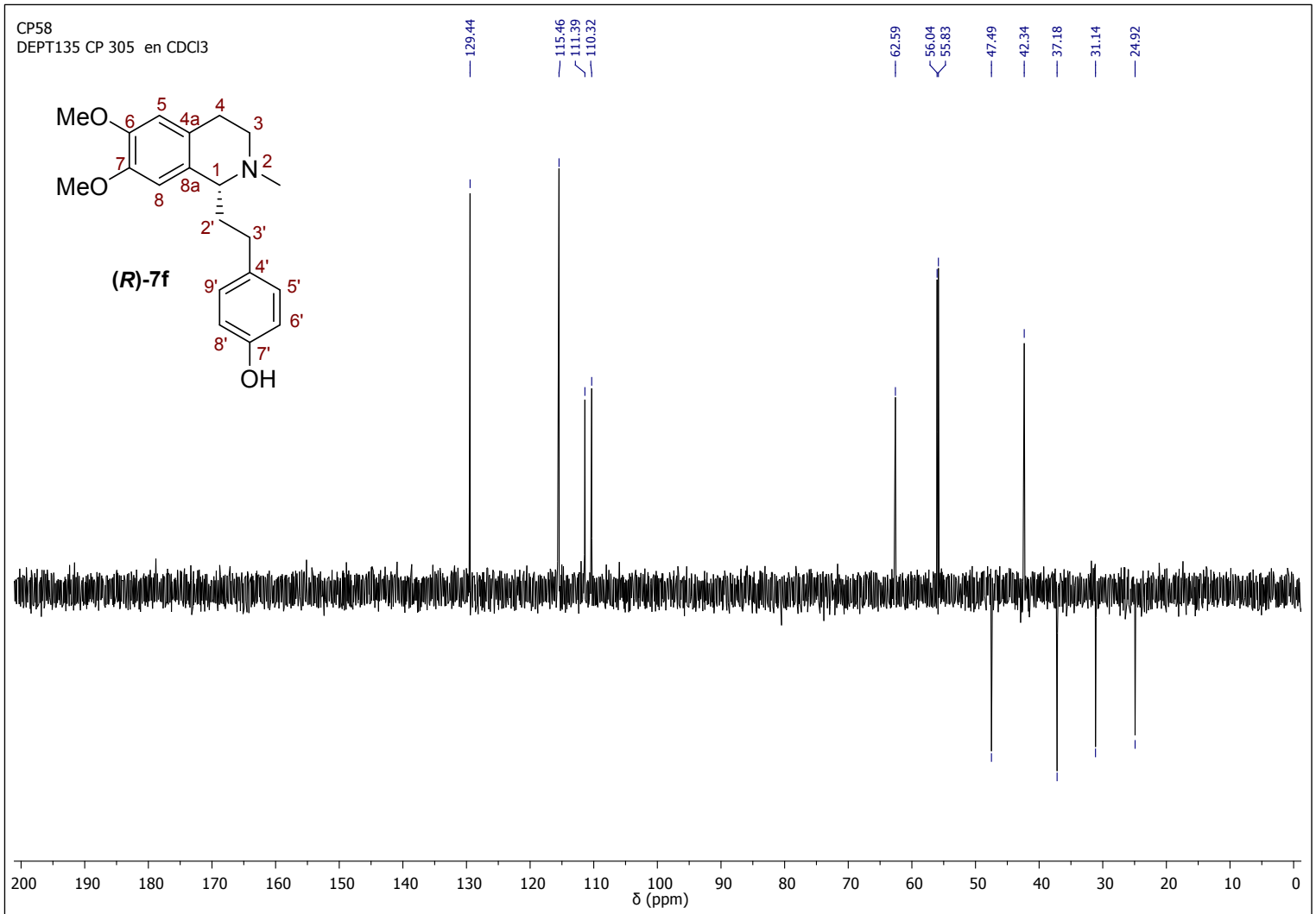

Figure S87. ${ }^{1} \mathrm{H}-\mathrm{NMR}$ spectrum of (R)-6,7-dimethoxy-1-(4-hydroxy-3-methoxyphenethyl)- $N$-methyl-1,2,3,4-tetrahydroisoquinoline (R)-7g. 


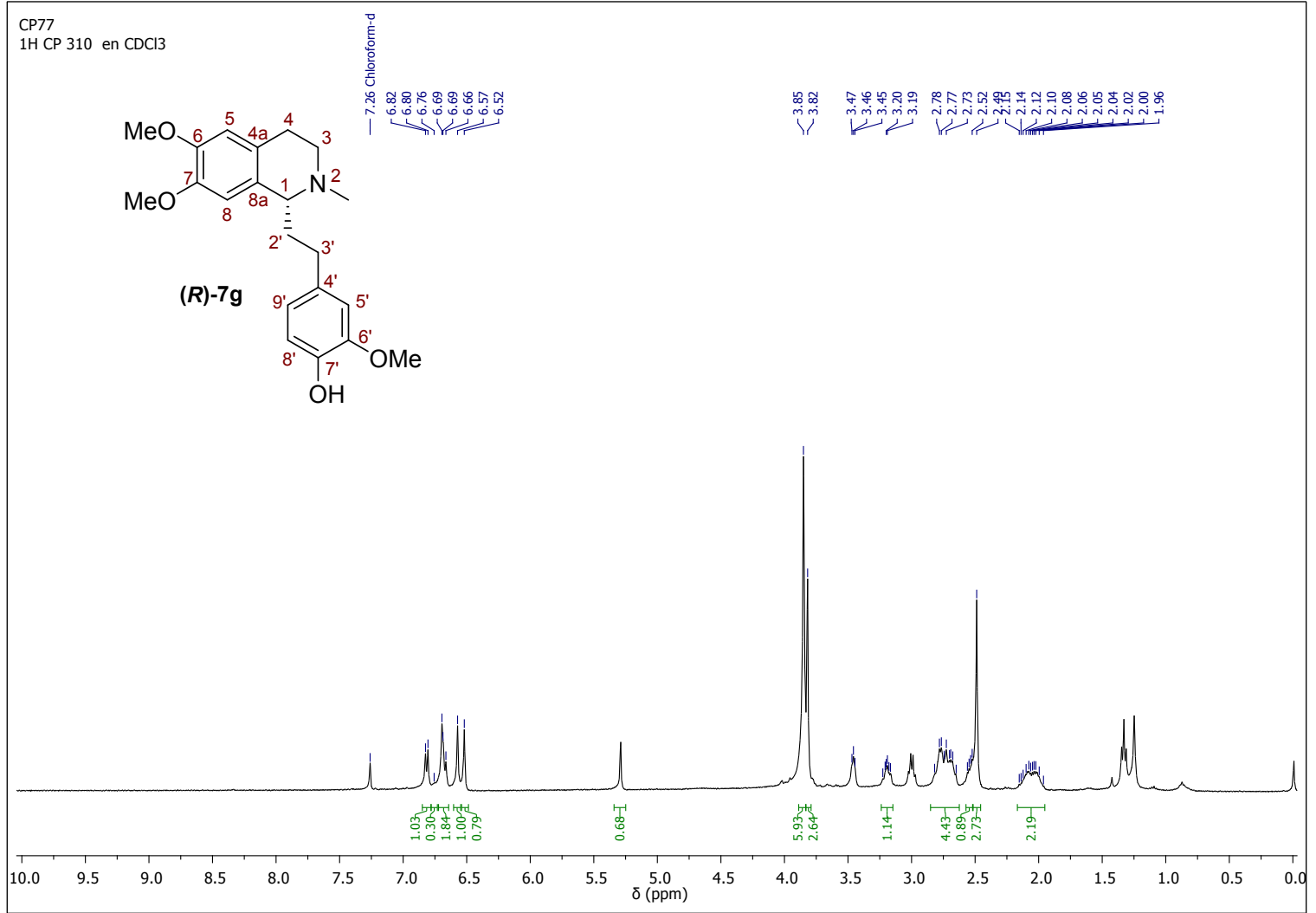

Figure S88. ${ }^{13} \mathrm{C}-\mathrm{NMR}$ spectrum of $(R)$-6,7-dimethoxy-1-(4-hydroxy-3-methoxyphenethyl)- $N$-methyl-1,2,3,4-tetrahydroisoquinoline (R)-7 $\mathbf{g}$

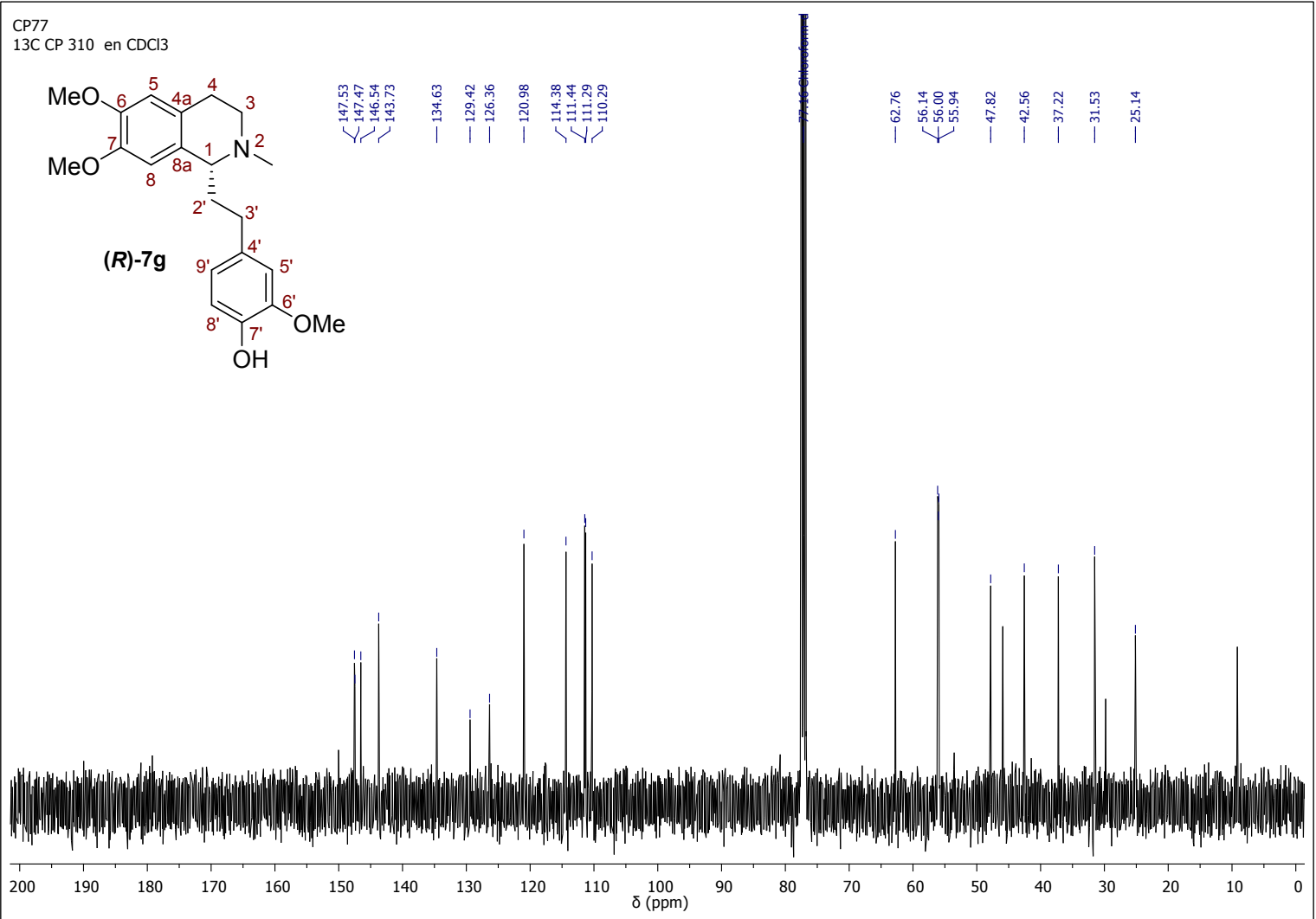

Figure S89. DEPT-135 spectrum of (R)-6,7-dimethoxy-1-(4-hydroxy-3-methoxyphenethyl)- $N$-methyl-1,2,3,4-tetrahydroisoquinoline (R)-7g. 


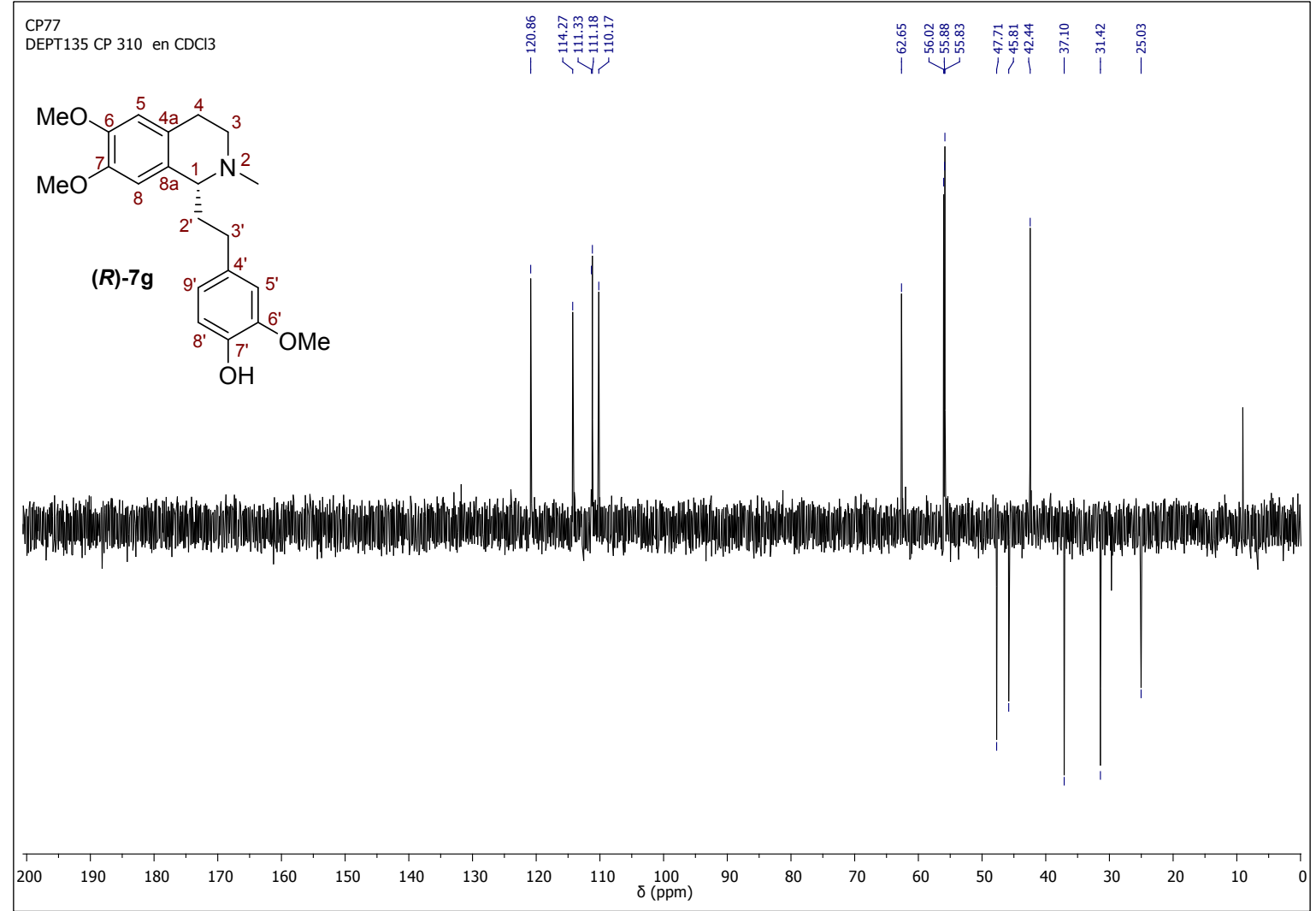

Figure S90. ${ }^{1} \mathrm{H}$-NMR spectrum of (R)-6,7-dimethoxy-1-(3-hydroxy-4-methoxyphenethyl)- $N$-methyl-1,2,3,4-tetrahydroisoquinoline (R)-7h

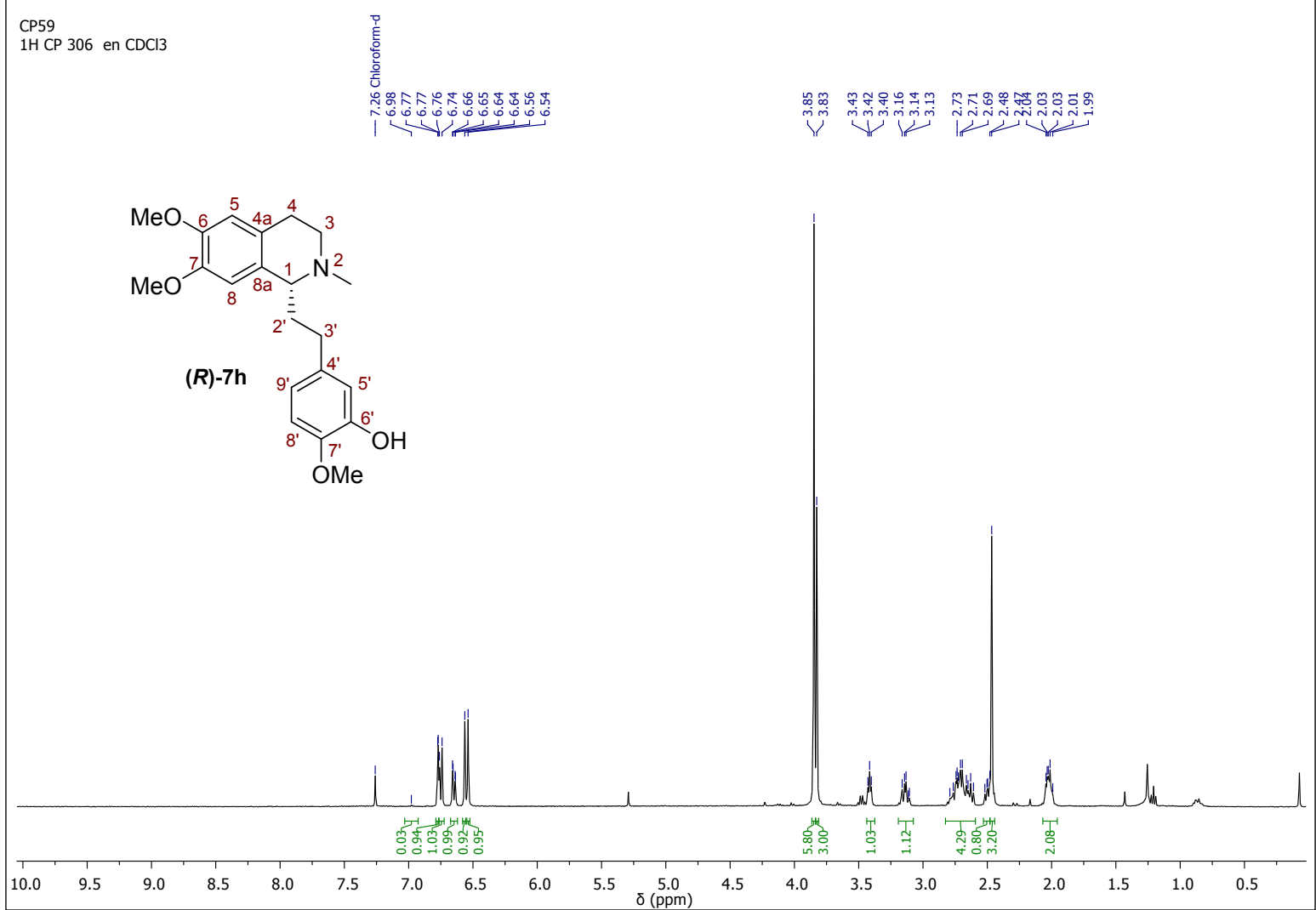

Figure S91. ${ }^{13} \mathrm{C}$-NMR spectrum of (R)-6,7-dimethoxy-1-(3-hydroxy-4-methoxyphenethyl)- $N$-methyl-1,2,3,4-tetrahydroisoquinoline (R)-7h . 


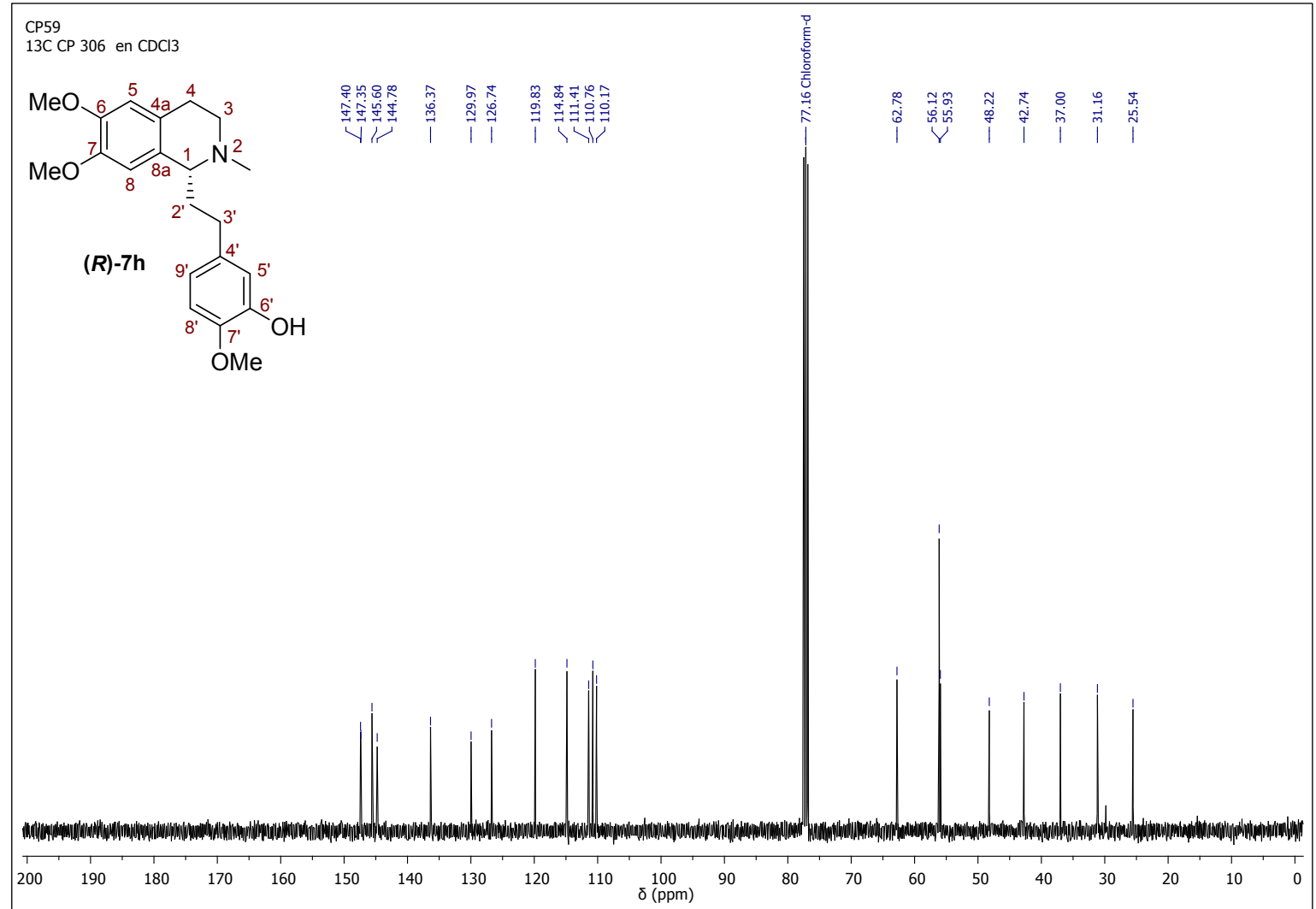

Figure S92. DEPT-135 spectrum of (R)-6,7-dimethoxy-1-(3-hydroxy-4-methoxyphenethyl)- $N$-methyl-1,2,3,4-tetrahydroisoquinoline (R)-7h

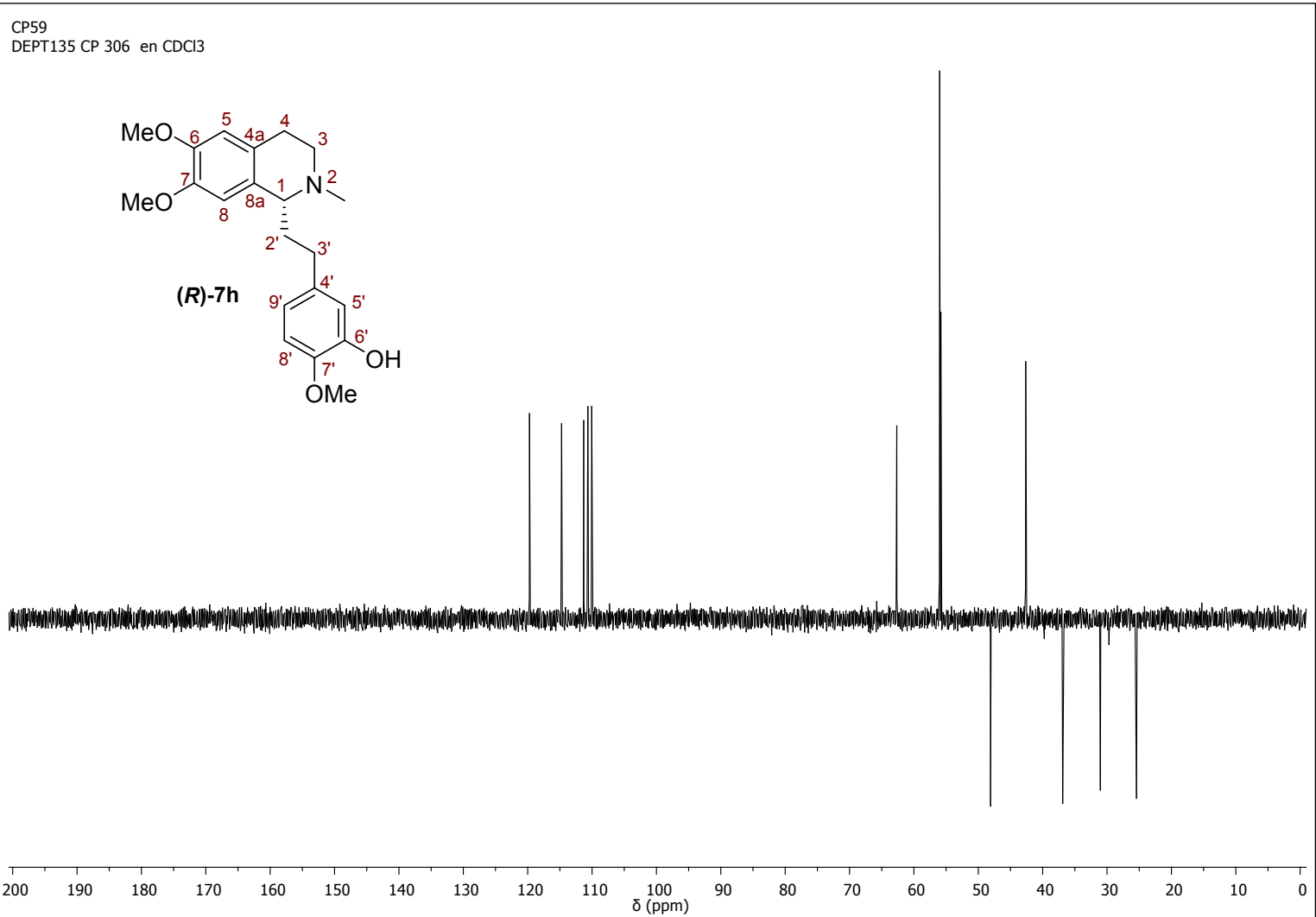

Figure S93. ${ }^{1} \mathrm{H}-\mathrm{NMR}$ spectrum of (R)-6,7-dimethoxy-1-(4-hydroxy-3,5-dimethoxyphenethyl)- $N$-methyl-1,2,3,4-tetrahydroisoquinoline (R)-7i. 


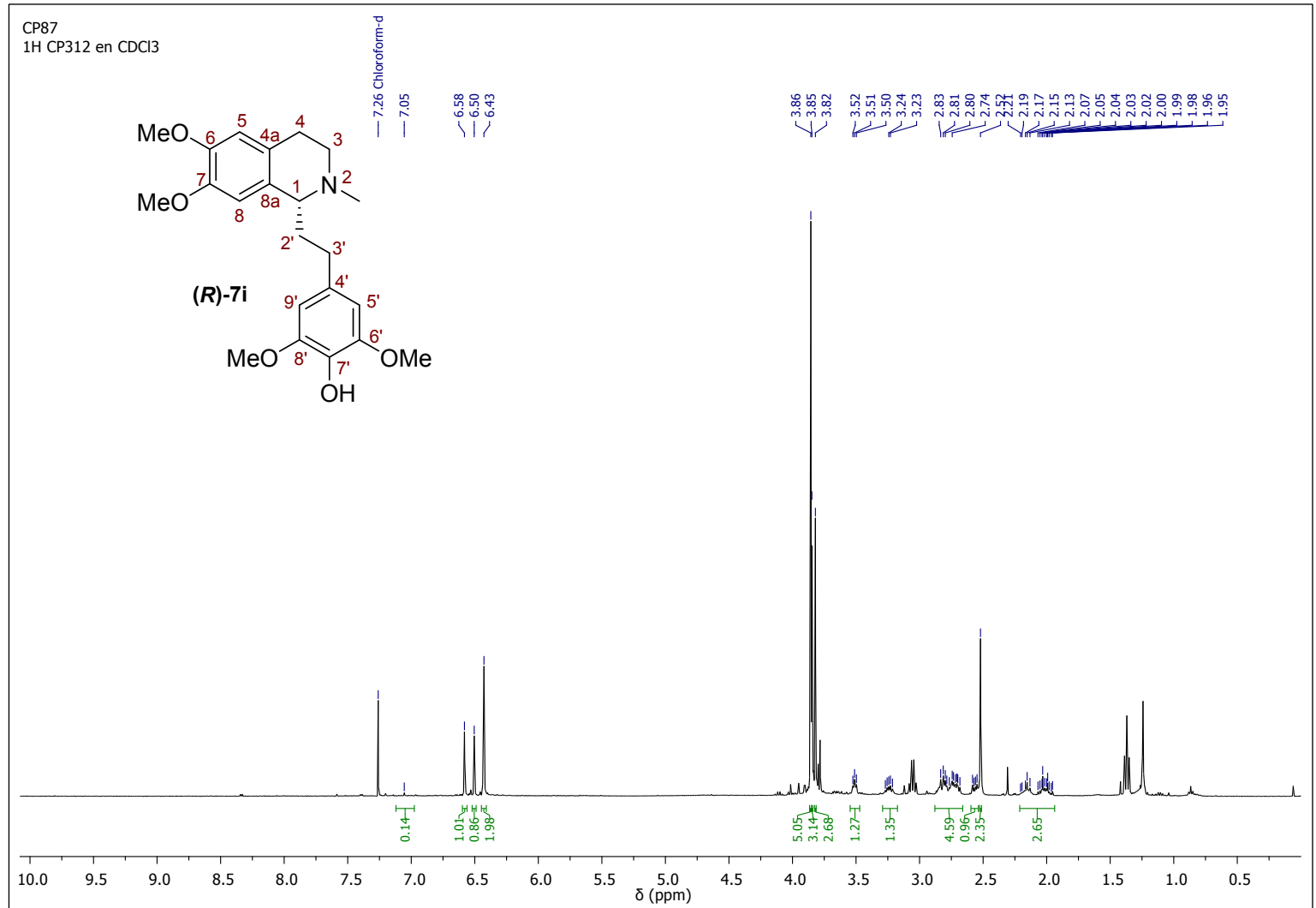

Figure S94. ${ }^{13} \mathrm{C}-\mathrm{NMR}$ spectrum of $(R)$-6,7-dimethoxy-1-(4-hydroxy-3,5-dimethoxyphenethyl)- $N$-methyl-1,2,3,4-tetrahydroisoquinoline (R)-7i.

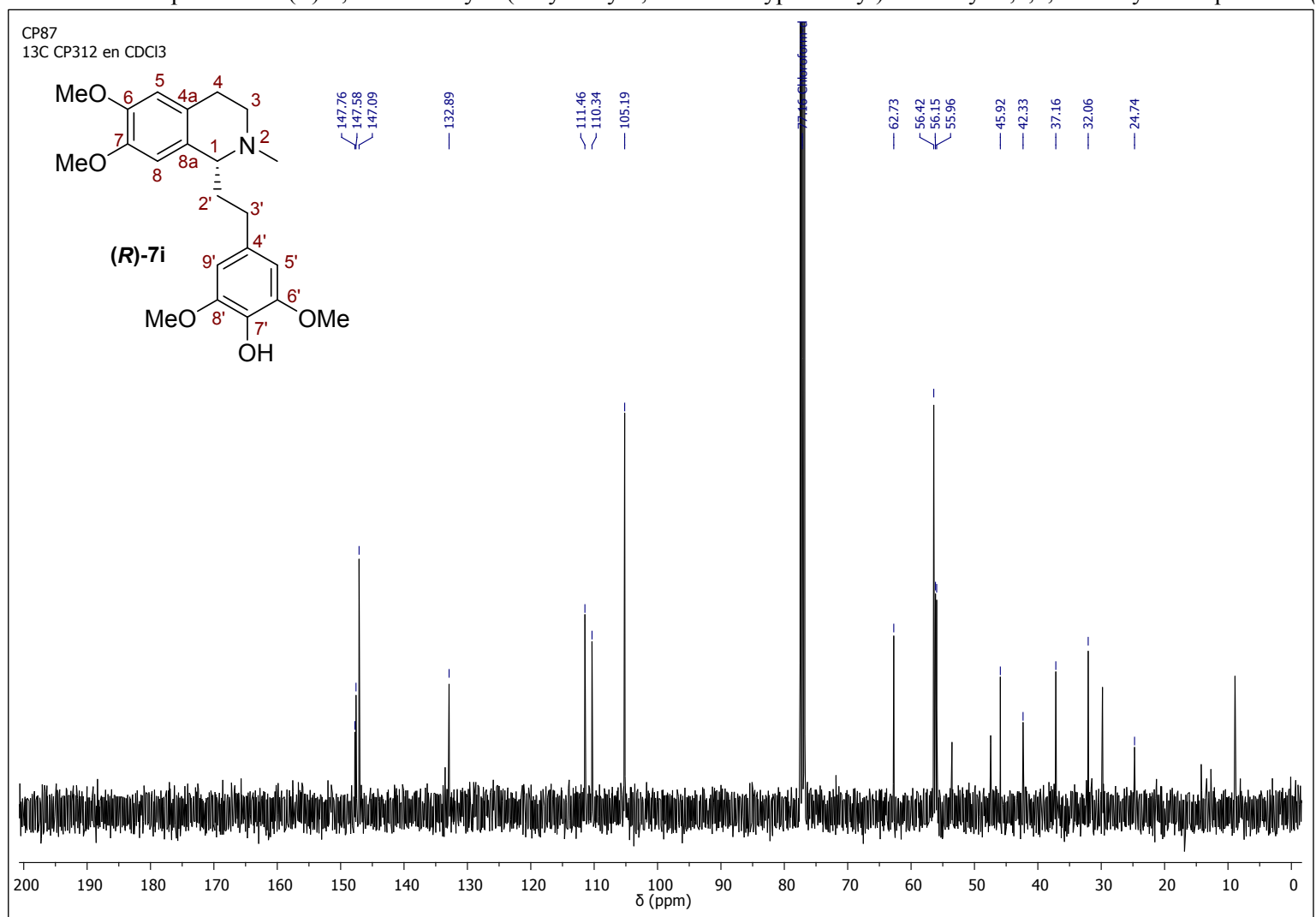

Figure S95. DEPT-135 spectrum of (R)-6,7-dimethoxy-1-(4-hydroxy-3,5-dimethoxyphenethyl)- $N$-methyl-1,2,3,4-tetrahydroisoquinoline (R)-7i. 


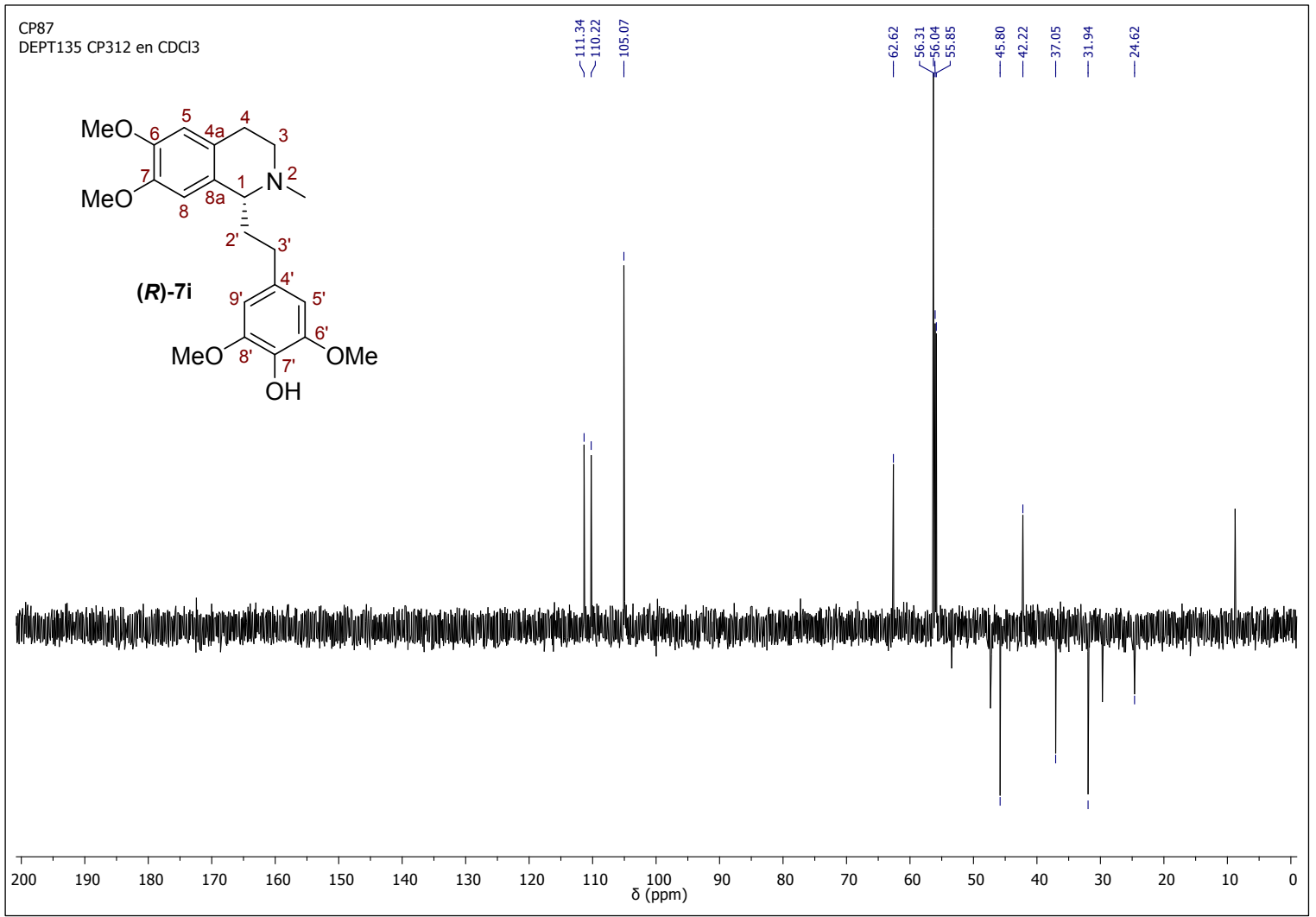

Figure S96. ${ }^{1} \mathrm{H}$-NMR spectrum of $(S)$-6,7-dimethoxy- $N$-methyl-1-phenethyl-1,2,3,4-tetrahydroisoquinoline $(\boldsymbol{S})$-7a .

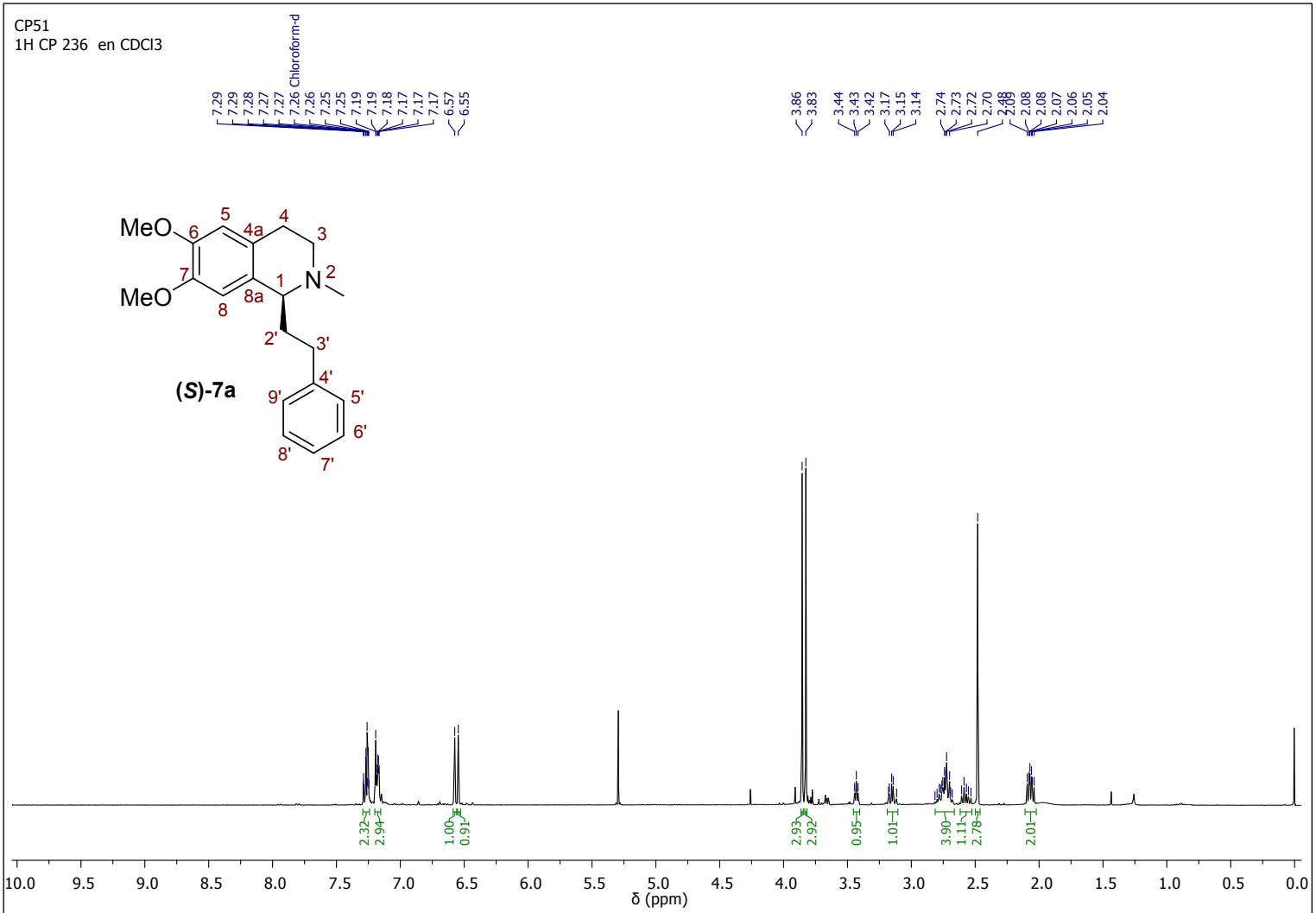

Figure S97. ${ }^{13} \mathrm{C}-\mathrm{NMR}$ spectrum of (S)-6,7-dimethoxy- $N$-methyl-1-phenethyl-1,2,3,4-tetrahydroisoquinoline (S)-7a. 


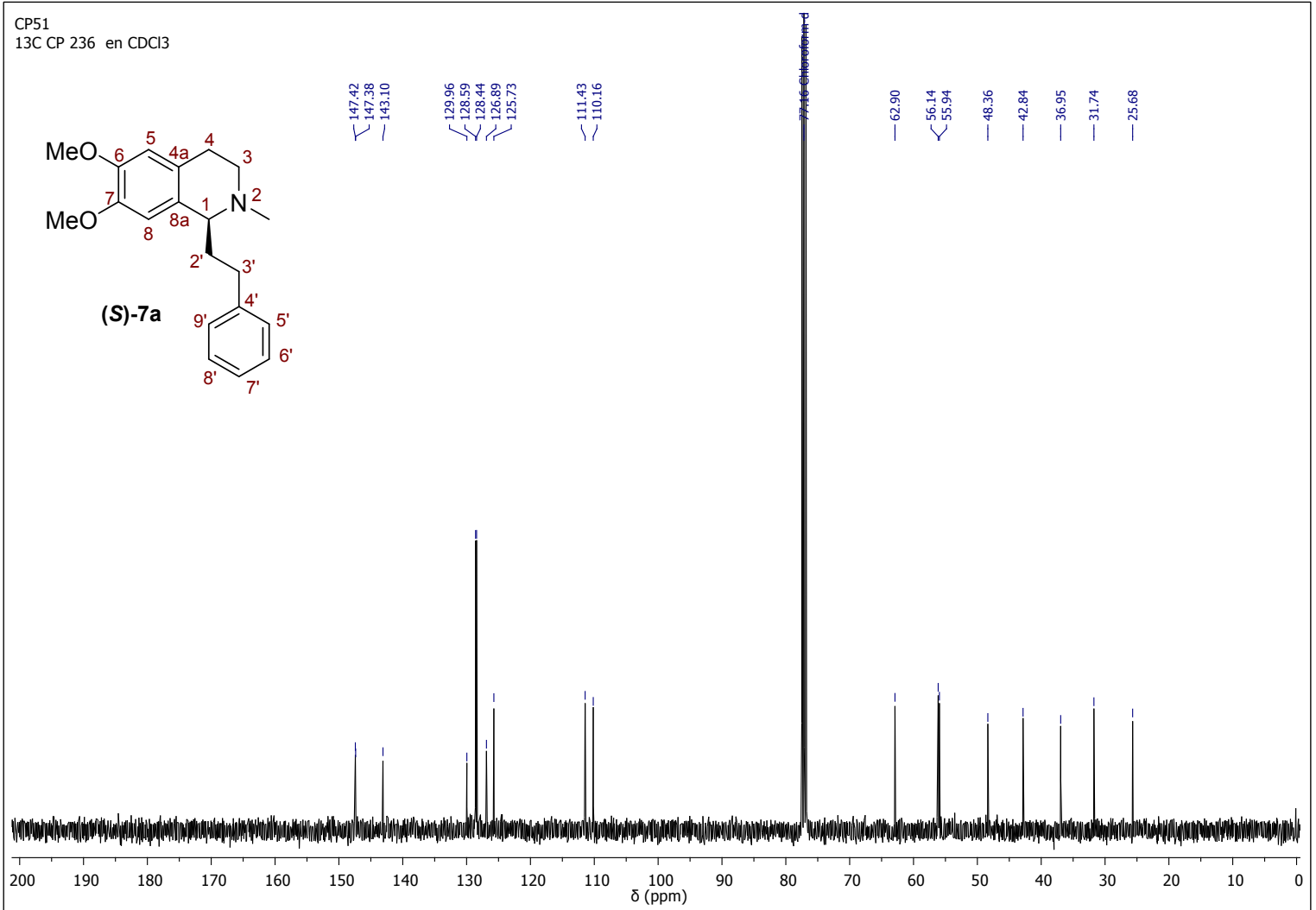

Figure S98. DEPT-135 spectrum of (S)-6,7-dimethoxy- $N$-methyl-1-phenethyl-1,2,3,4-tetrahydroisoquinoline (S)-7a.

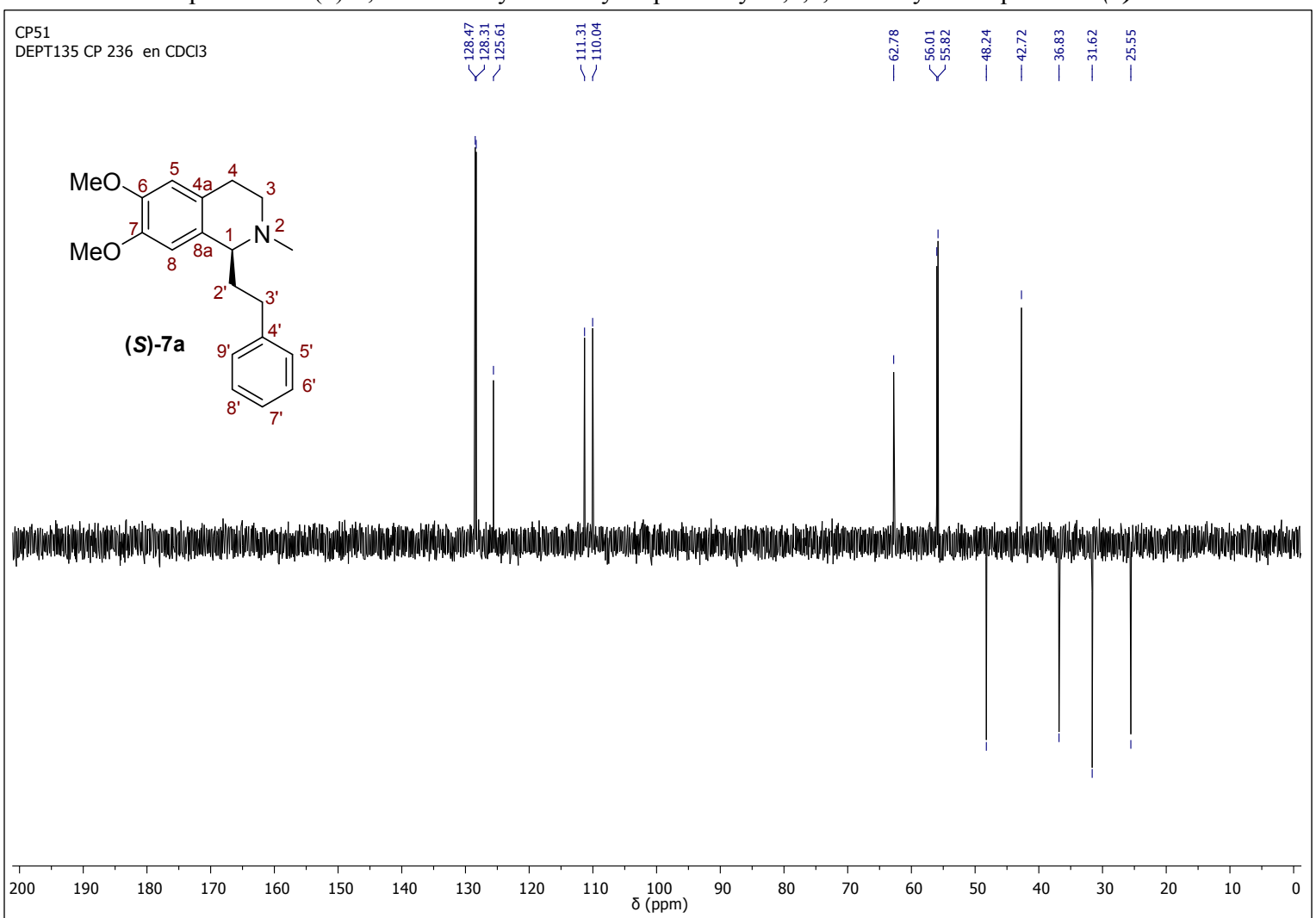

Figure S99. ${ }^{1} \mathrm{H}$-NMR spectrum of (S)-6,7-dimethoxy-1-(4-methoxyphenethyl)- $N$-methyl-1,2,3,4-tetrahydroisoquinoline (S)-7b. 


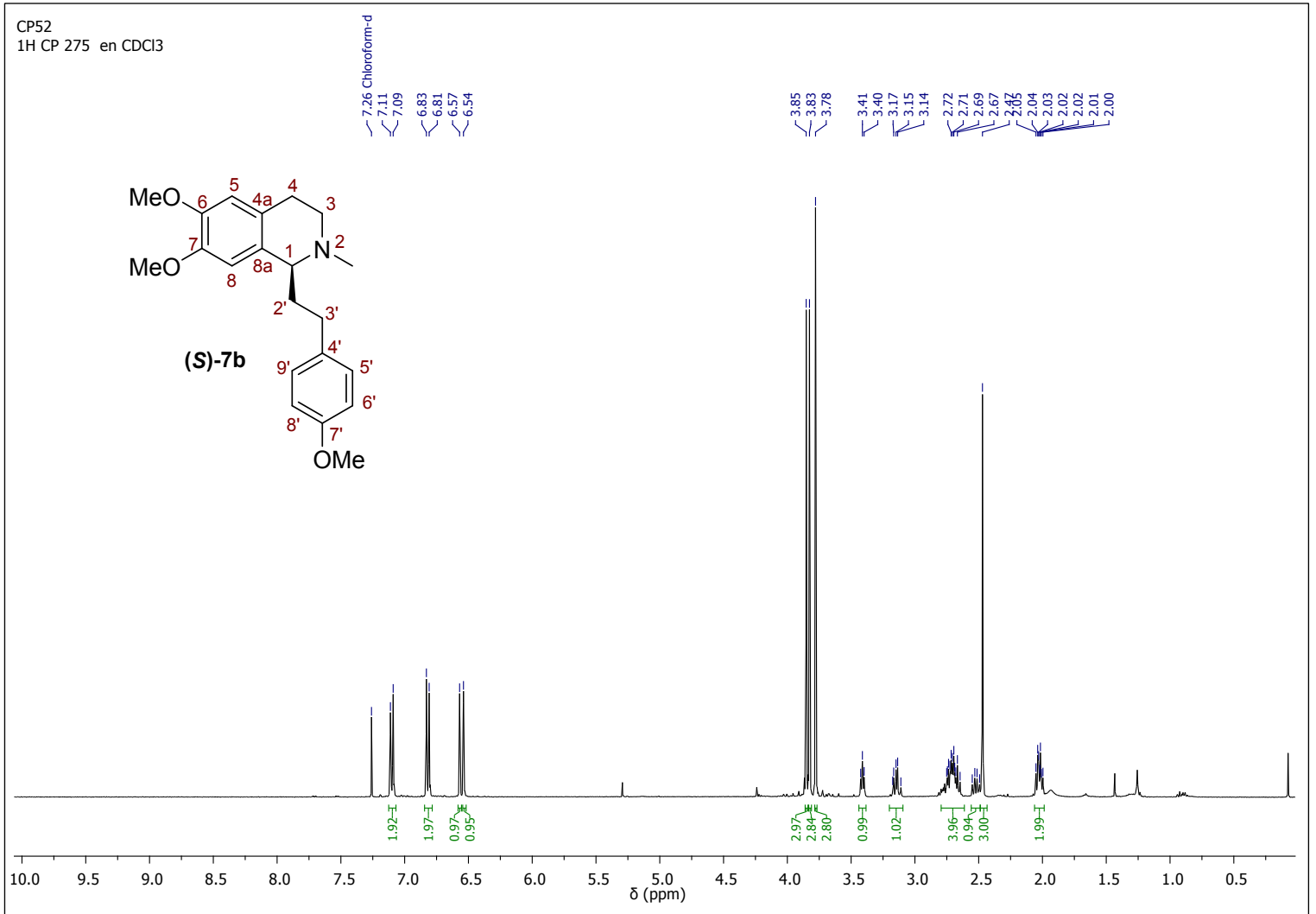

Figure S100. ${ }^{13} \mathrm{C}$-NMR spectrum of (S)-6,7-dimethoxy-1-(4-methoxyphenethyl)- $N$-methyl-1,2,3,4-tetrahydroisoquinoline (S)-7b.

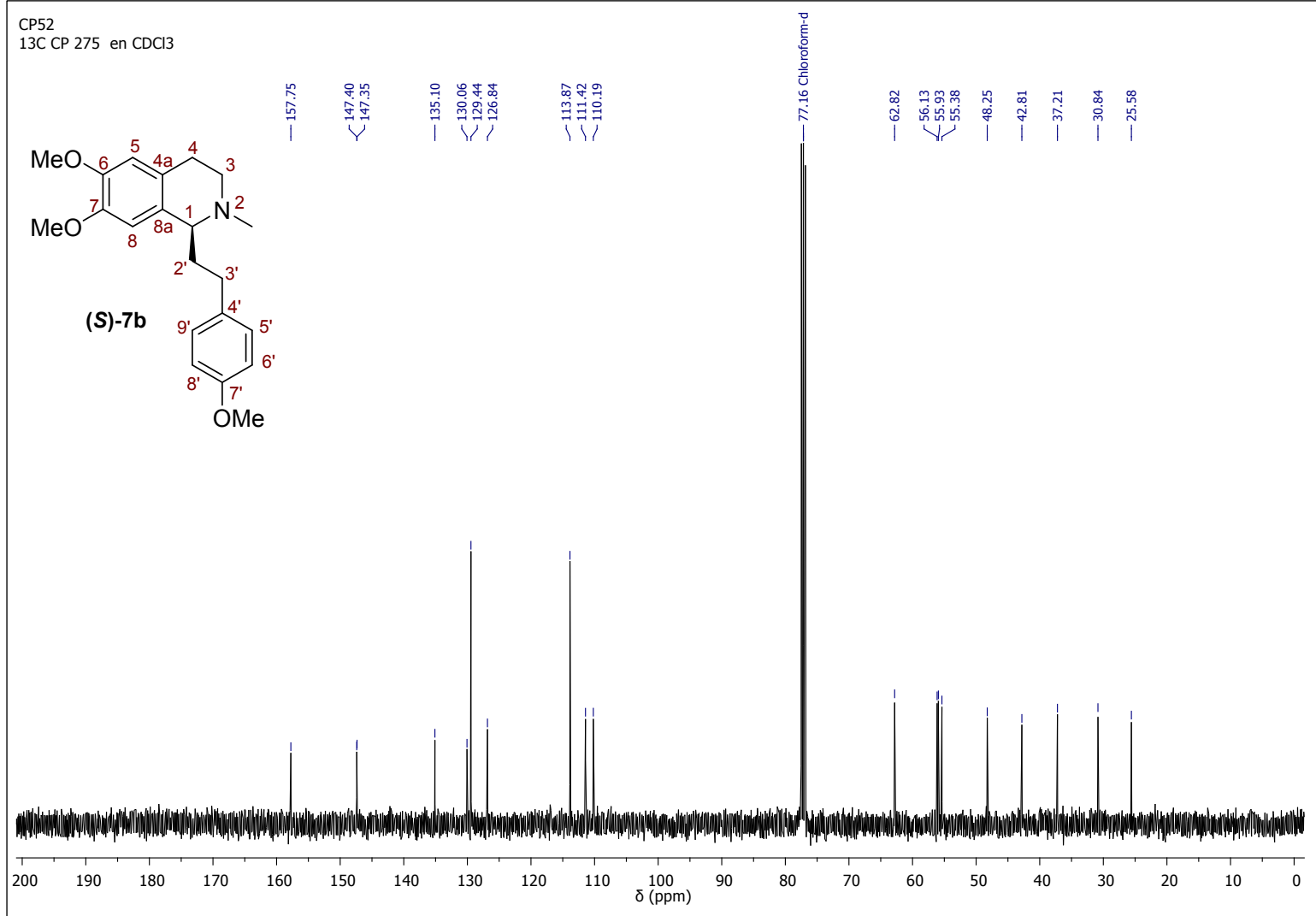

Figure S101. DEPT-135 spectrum of (S)-6,7-dimethoxy-1-(4-methoxyphenethyl)- $N$-methyl-1,2,3,4-tetrahydroisoquinoline (S)-7b. 


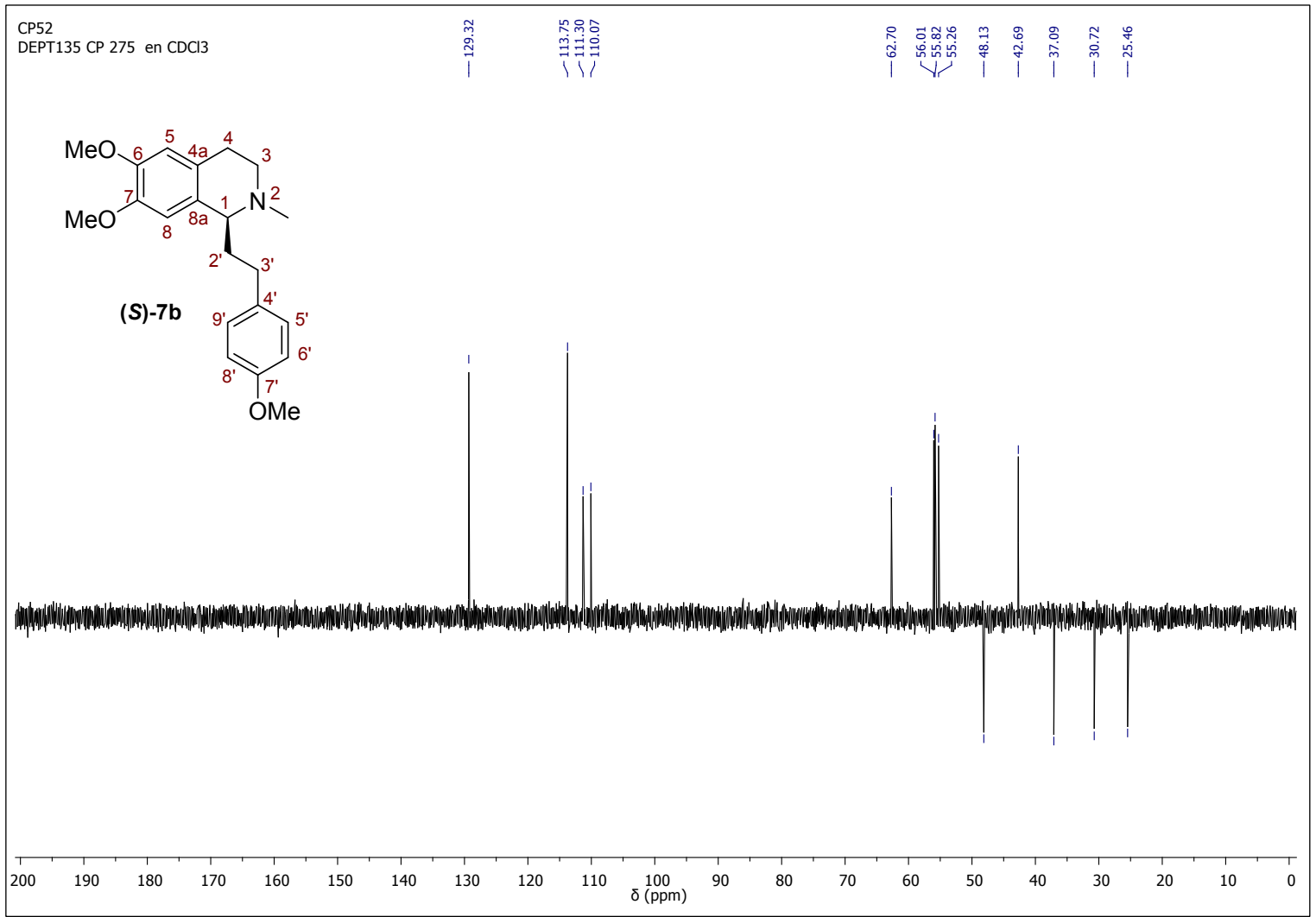

Figure S102. ${ }^{1} \mathrm{H}-\mathrm{NMR}$ spectrum of (S)-6,7-dimethoxy-1-(3,4-dimethoxyphenethyl)- $N$-methyl-1,2,3,4-tetrahydroisoquinoline (S)-7c

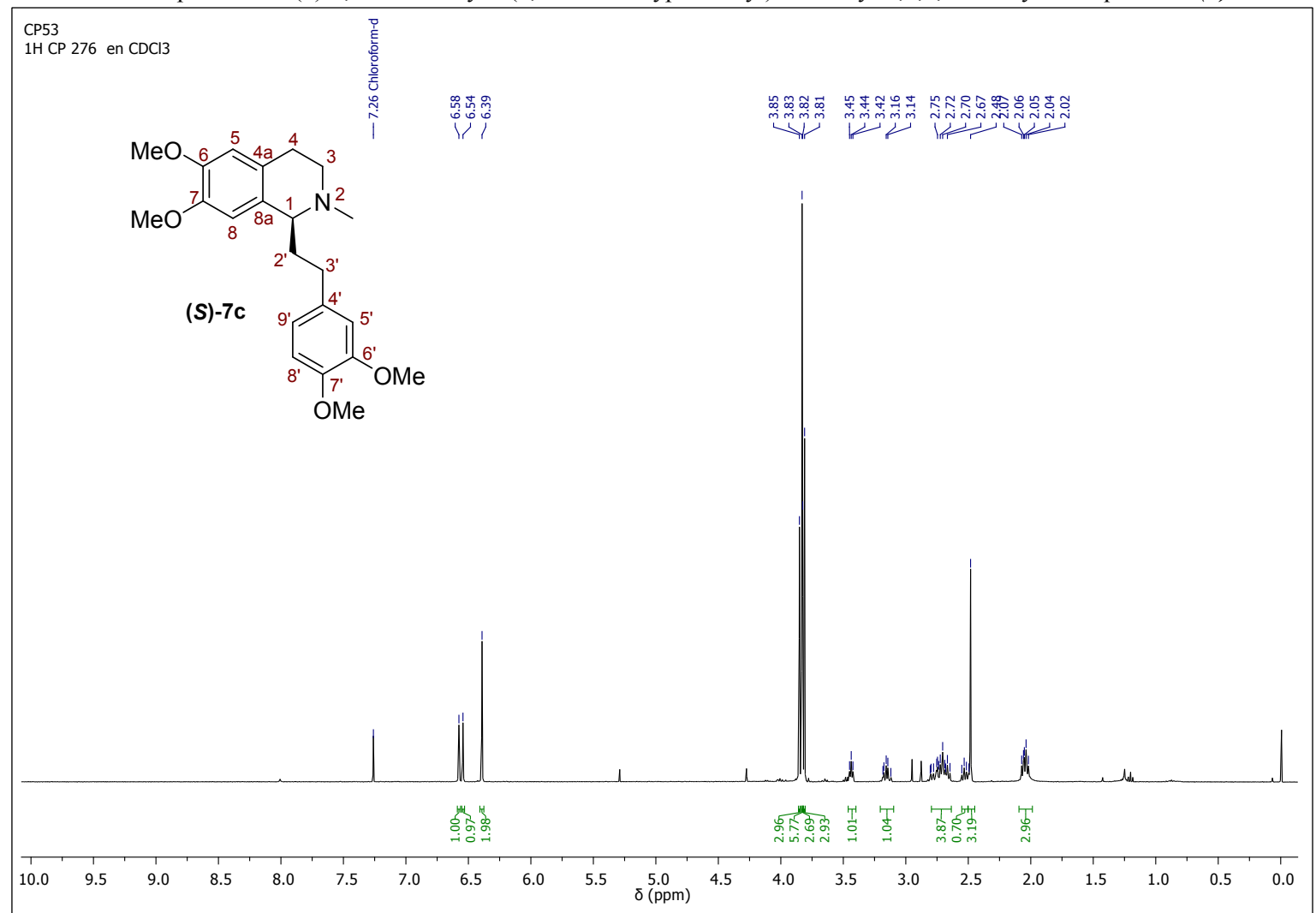

Figure S103. ${ }^{13} \mathrm{C}$-NMR spectrum of (S)-6,7-dimethoxy-1-(3,4-dimethoxyphenethyl)- $N$-methyl-1,2,3,4-tetrahydroisoquinoline (S)-7c . 


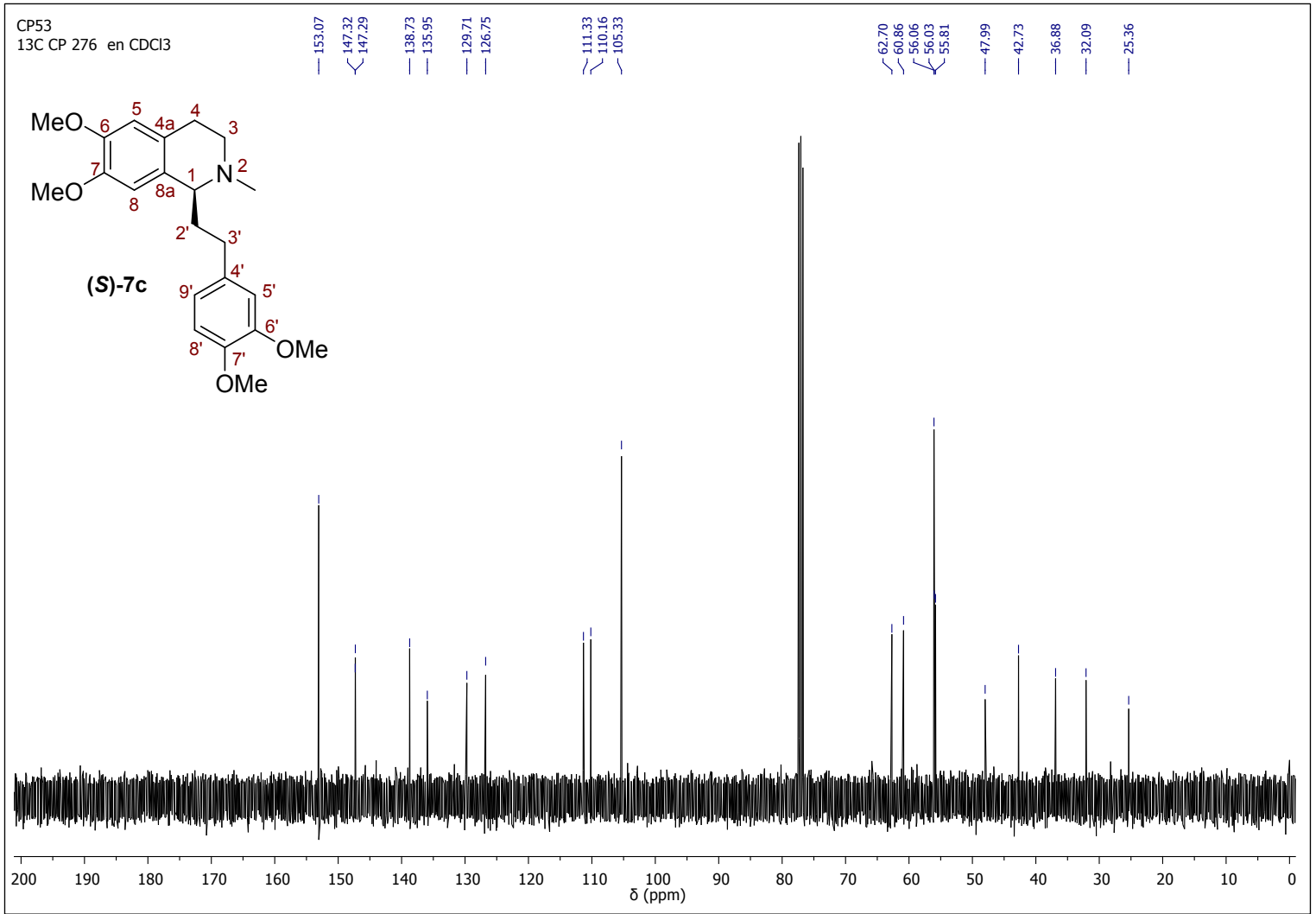

Figure S104. DEPT-135 spectrum of (S)-6,7-dimethoxy-1-(3,4-dimethoxyphenethyl)- $N$-methyl-1,2,3,4-tetrahydroisoquinoline (S)-7c.

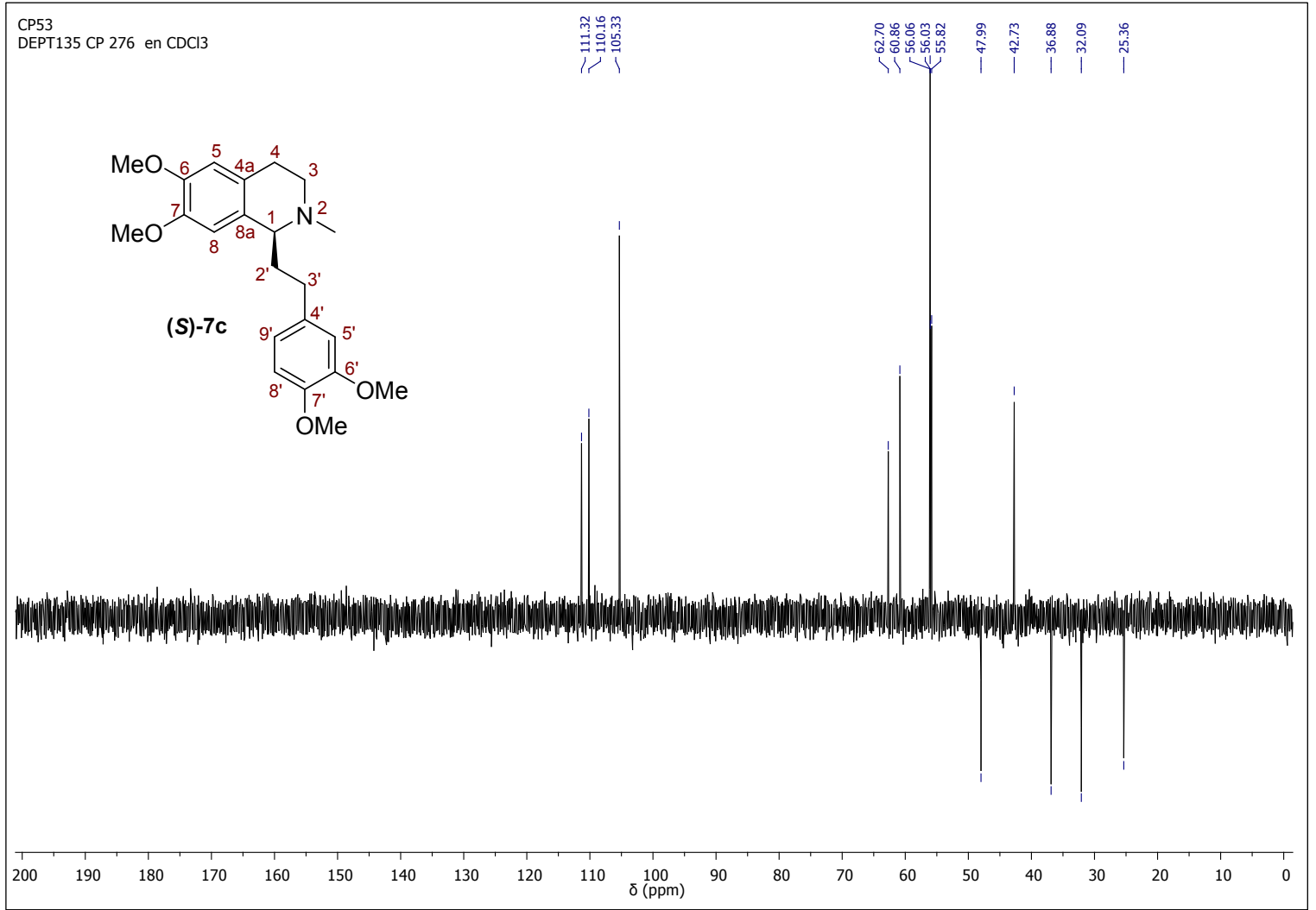

Figure S105. ${ }^{1} \mathrm{H}-\mathrm{NMR}$ spectrum of (S)-6,7-dimethoxy-1-(3,4,5-trimethoxyphenethyl)- $N$-methyl-1,2,3,4-tetrahydroisoquinoline (S)-7d. 


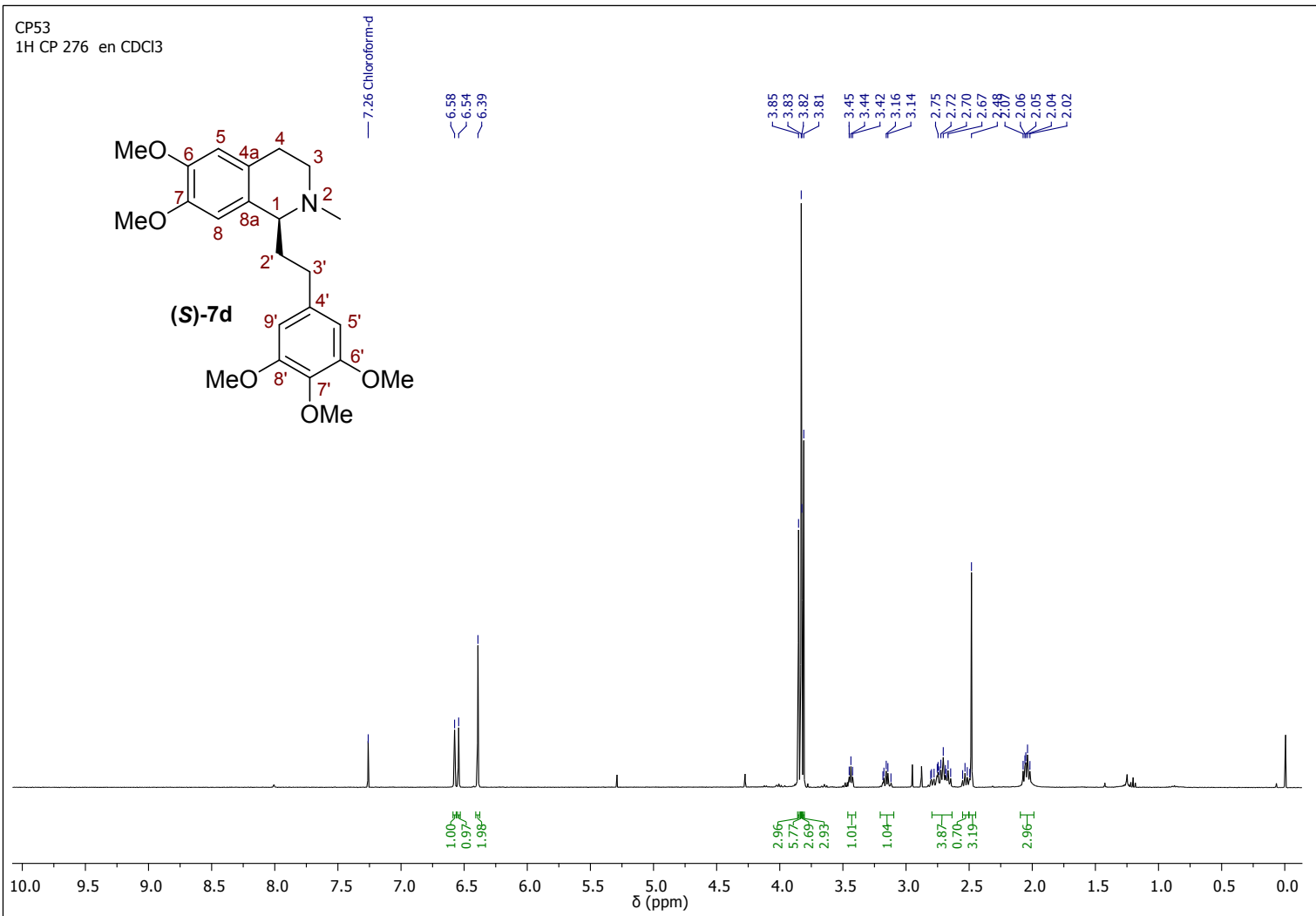

Figure S106. ${ }^{13} \mathrm{C}-\mathrm{NMR}$ spectrum of (S)-6,7-dimethoxy-1-(3,4,5-trimethoxyphenethyl)- $N$-methyl-1,2,3,4-tetrahydroisoquinoline (S)-7d .

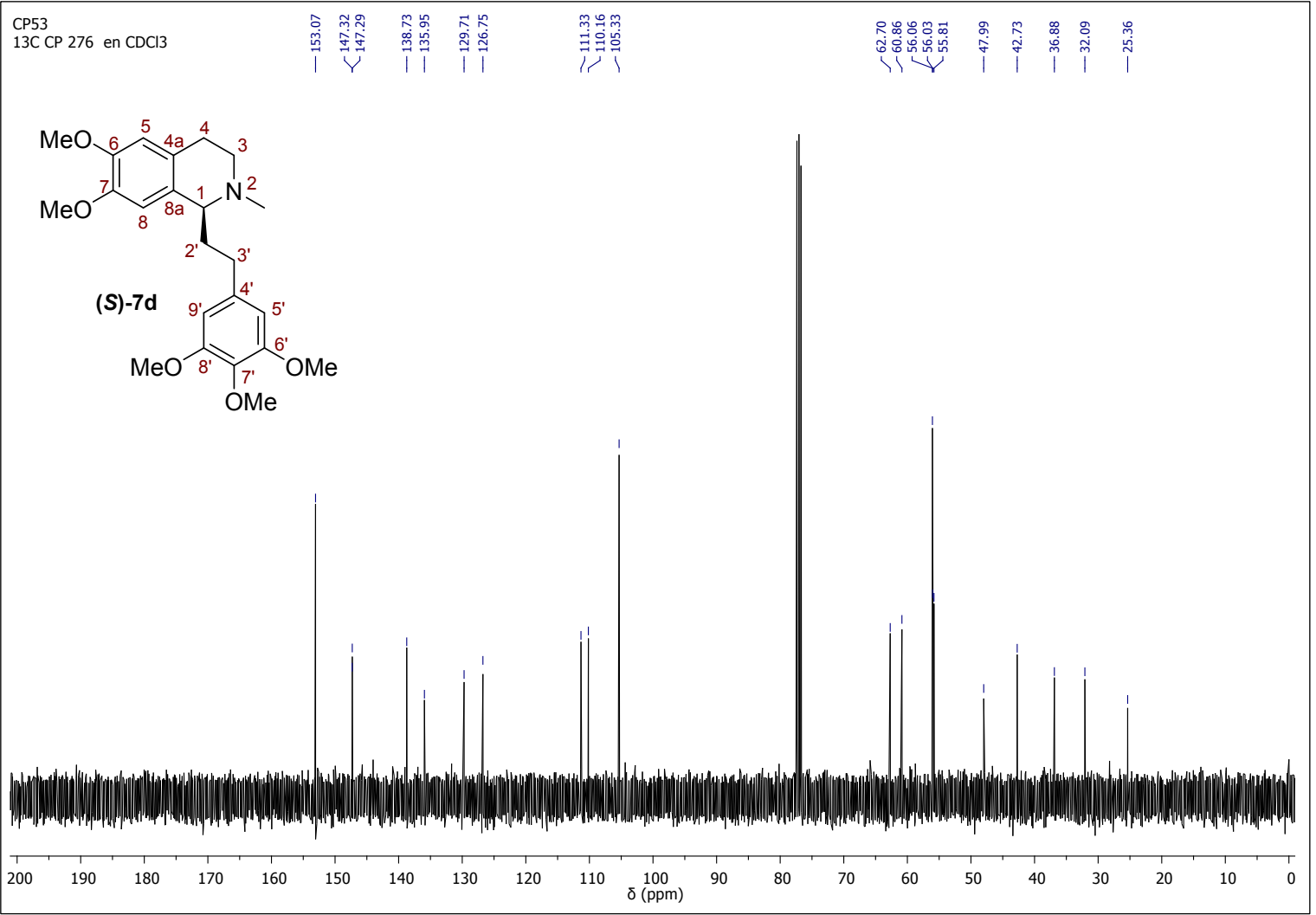

Figure S107. DEPT-135 spectrum of (S)-6,7-dimethoxy-1-(3,4,5-trimethoxyphenethyl)- $N$-methyl-1,2,3,4-tetrahydroisoquinoline (S)-7d . 


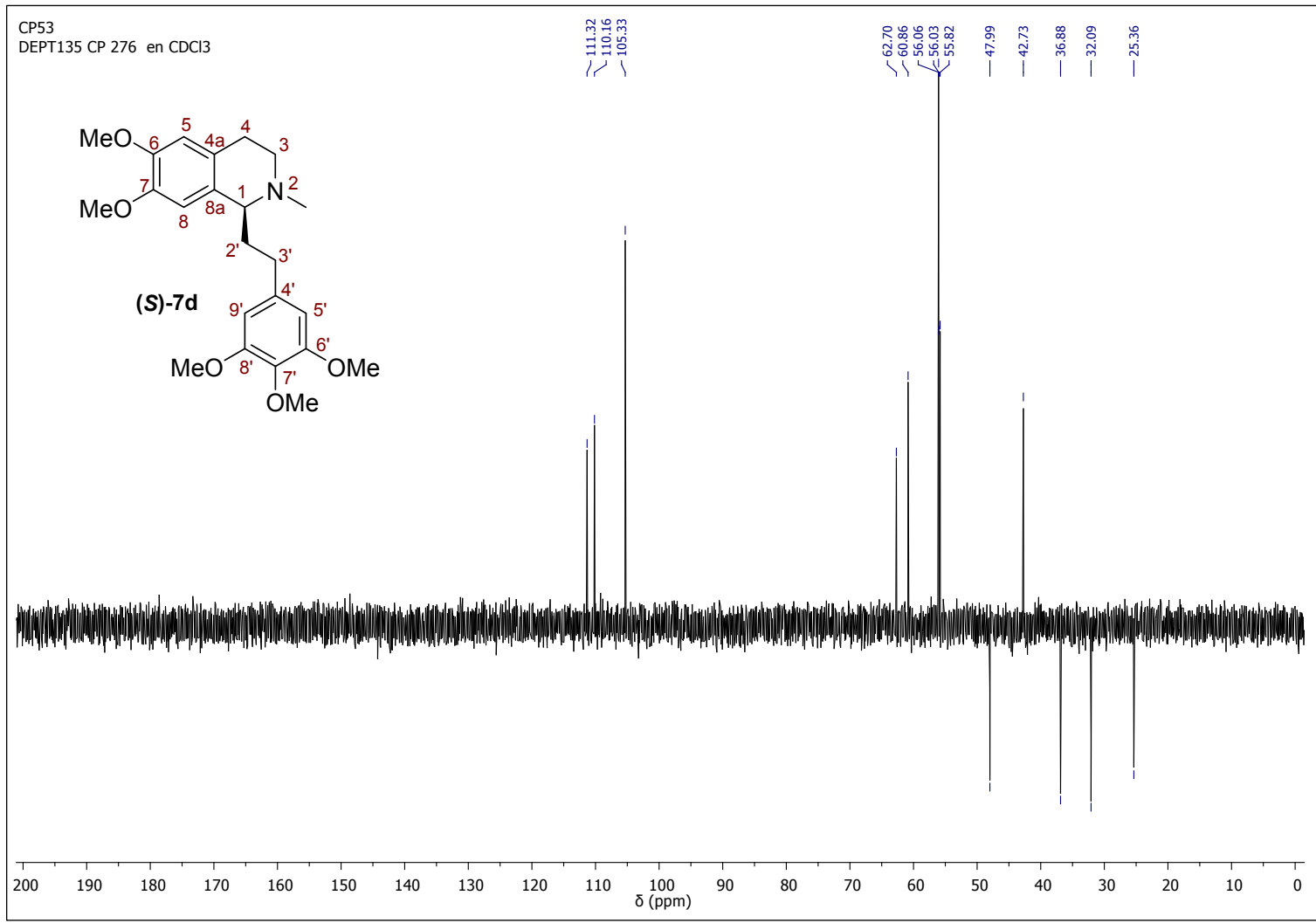

Figure S108. ${ }^{1} \mathrm{H}-\mathrm{NMR}$ spectrum of (S)-6,7-dimethoxy-1-(3,4-methylendioxyphenethyl)- $N$-methyl-1,2,3,4-tetrahydroisoquinoline (S)-7e

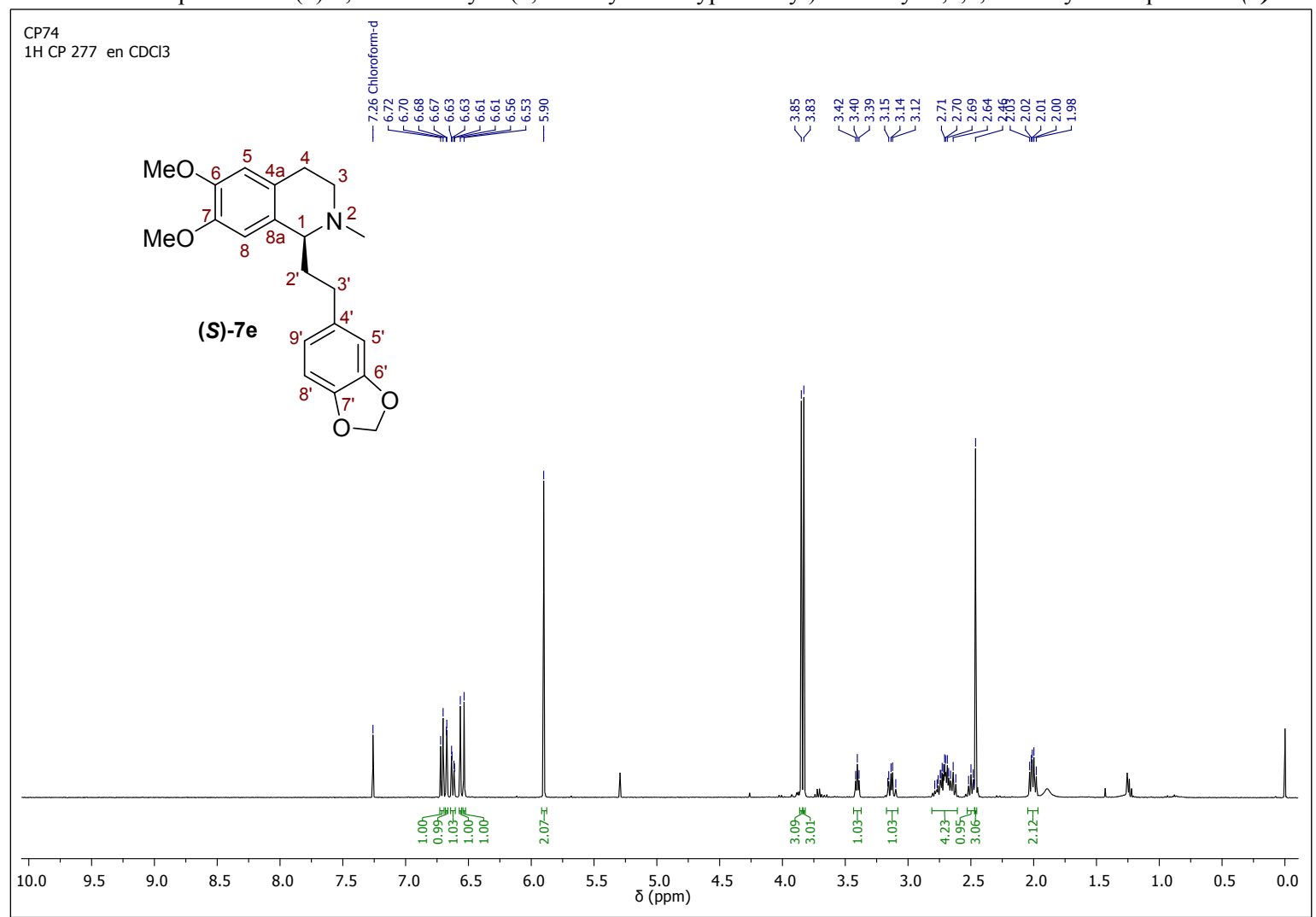

Figure S109. ${ }^{13} \mathrm{C}$-NMR spectrum of (S)-6,7-dimethoxy-1-(3,4-methylendioxyphenethyl)- $N$-methyl-1,2,3,4-tetrahydroisoquinoline (S)-7e 


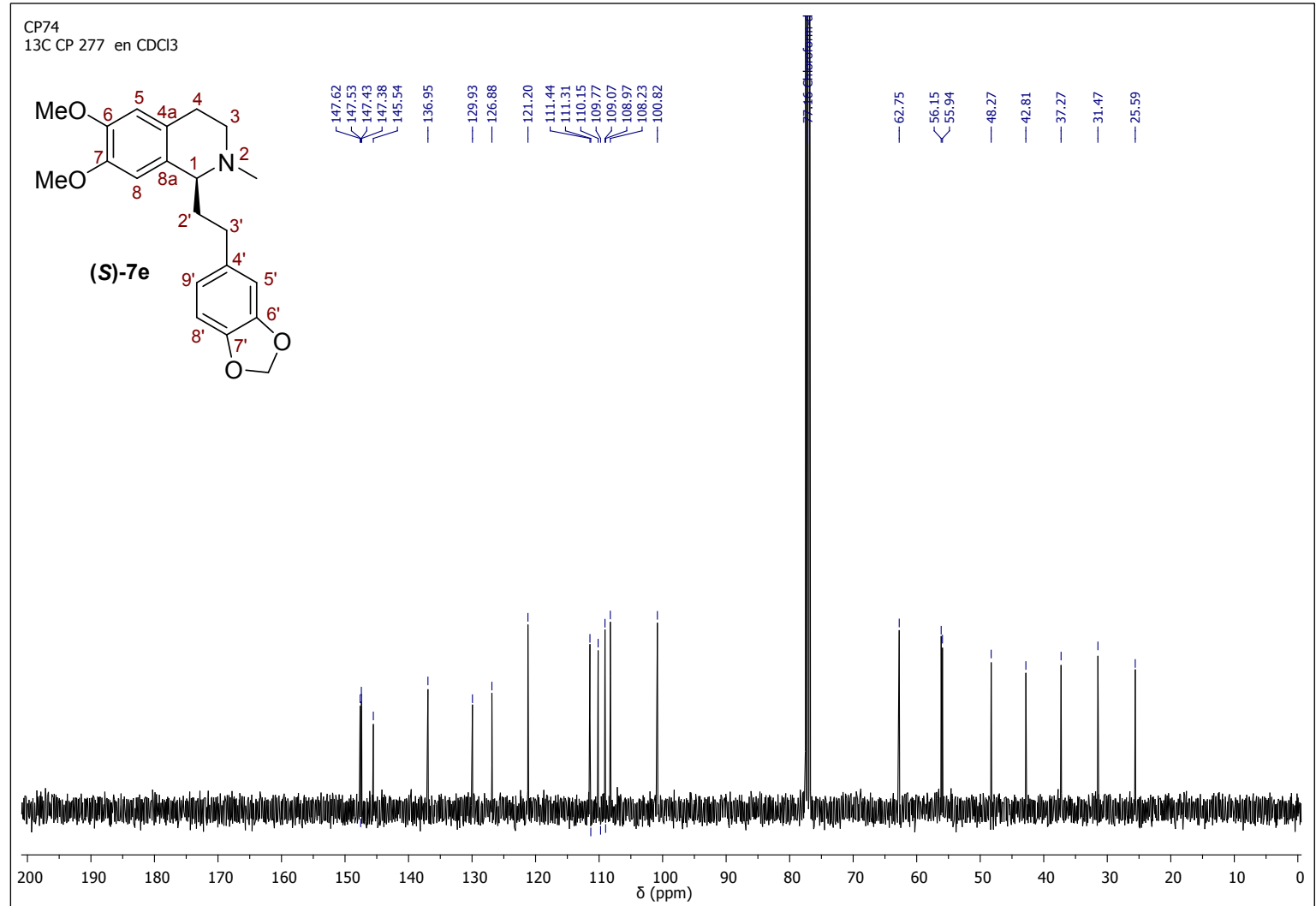

Figure S110. DEPT-135 spectrum of (S)-6,7-dimethoxy-1-(3,4-methylendioxyphenethyl)- $N$-methyl-1,2,3,4-tetrahydroisoquinoline (S)-7e .

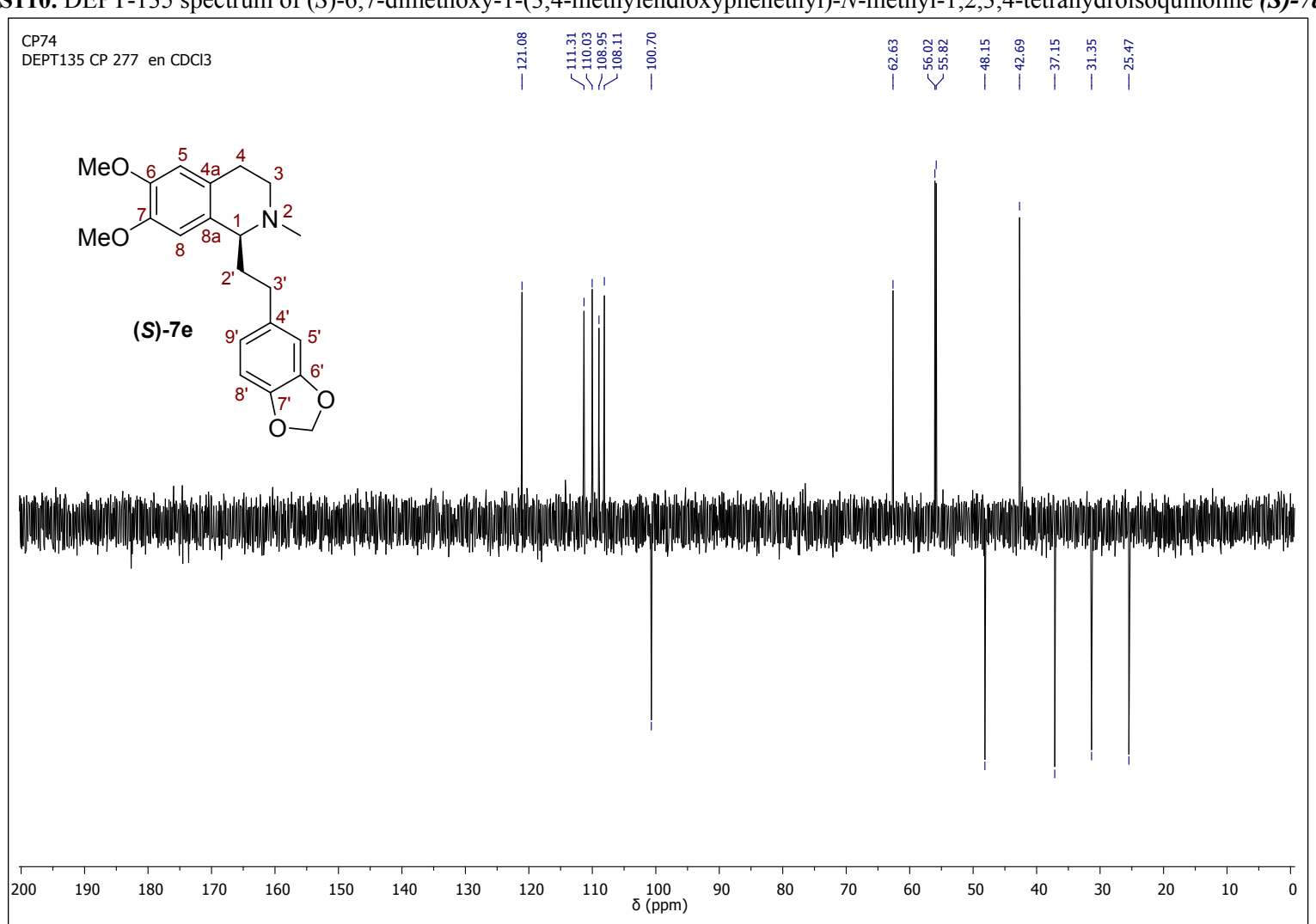

Figure S111. ${ }^{1} \mathrm{H}-\mathrm{NMR}$ spectrum of (S)-6,7-dimethoxy-1-(4-hydroxyphenethyl)- $N$-methyl-1,2,3,4-tetrahydroisoquinoline (R)-7f . 


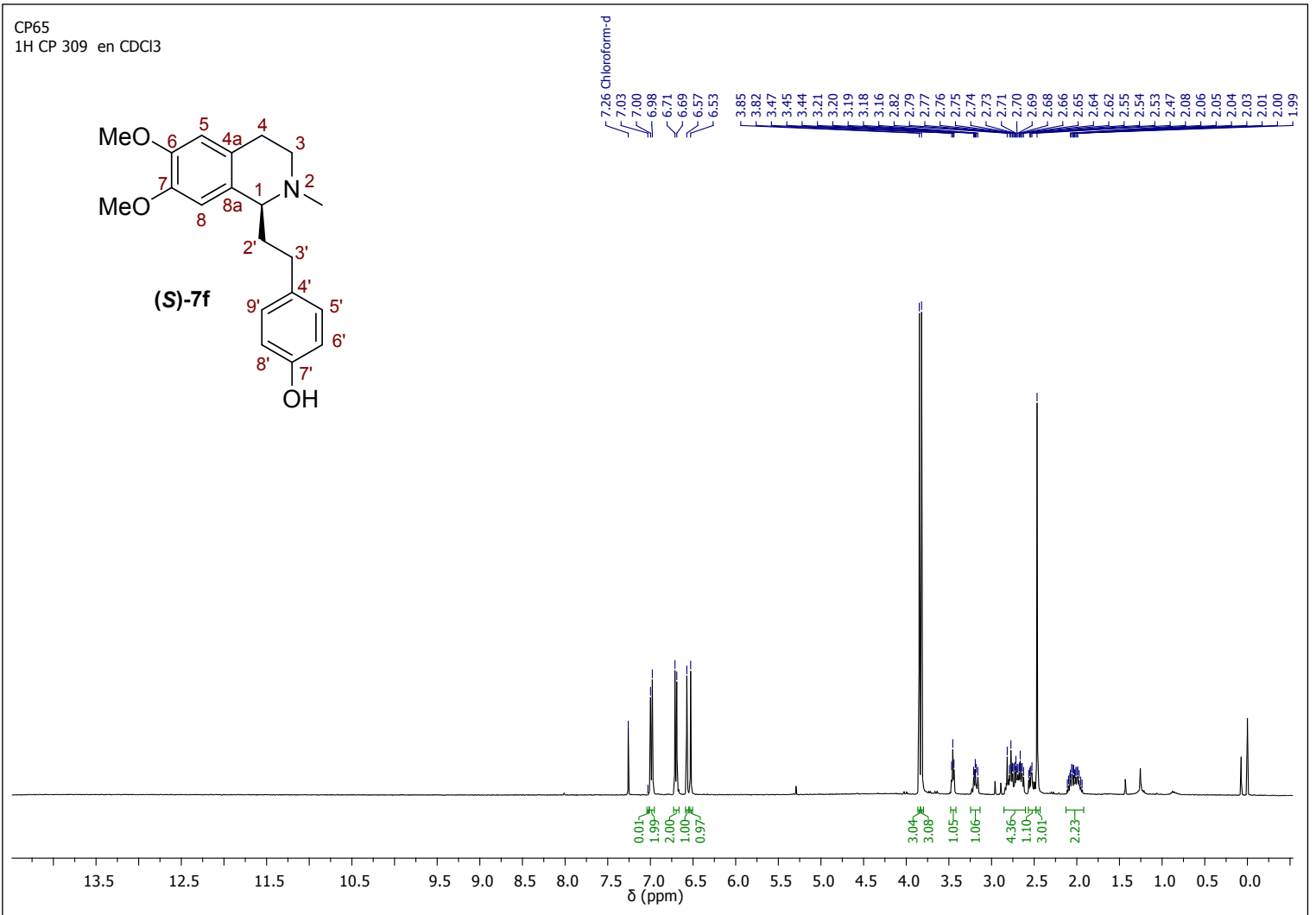

Figure S112. ${ }^{13} \mathrm{C}$-NMR spectrum of (S)-6,7-dimethoxy-1-(4-hydroxyphenethyl)- $N$-methyl-1,2,3,4-tetrahydroisoquinoline (S)-7f.

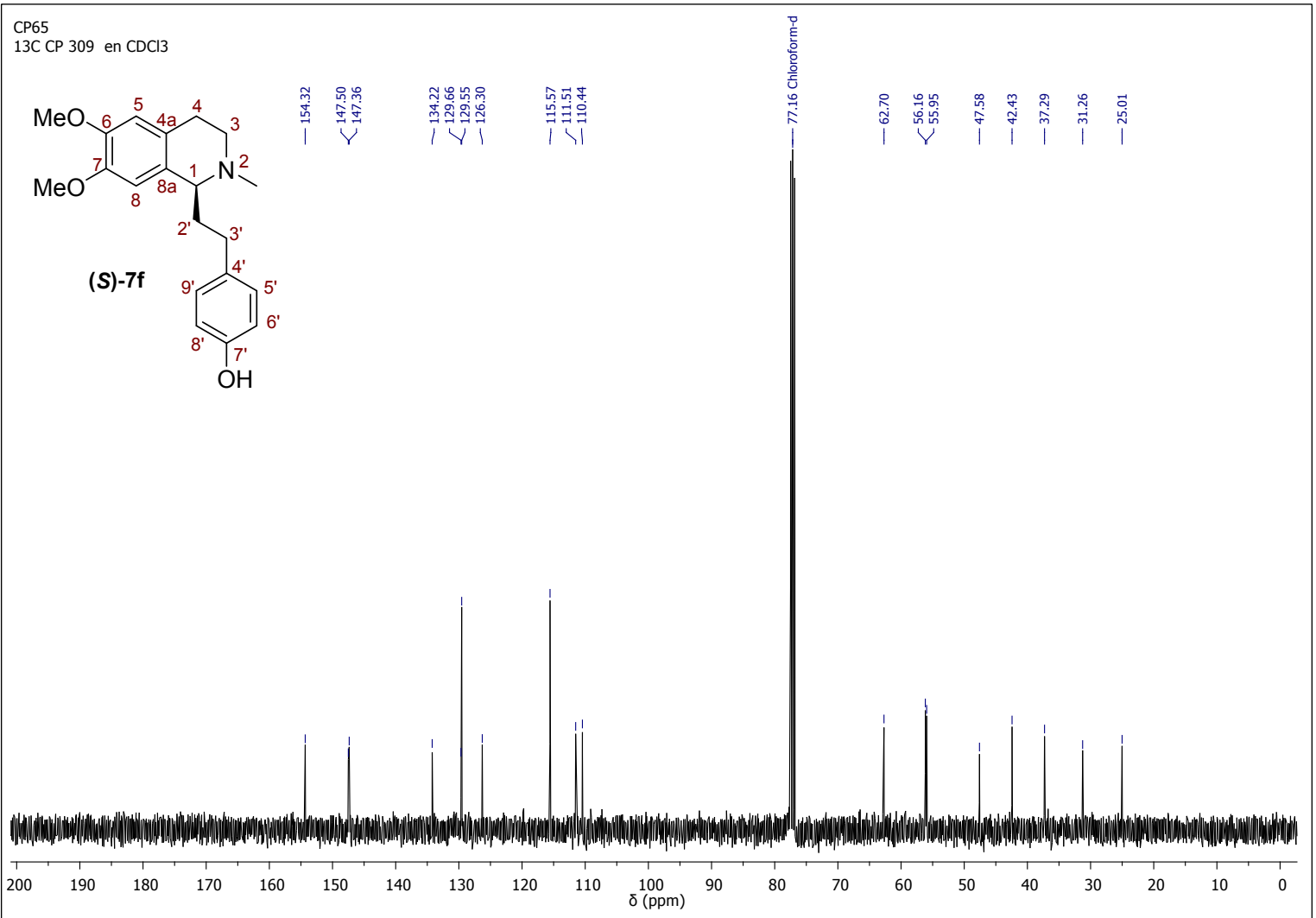

Figure S113. DEPT-135 spectrum of (S)-6,7-dimethoxy-1-(4-hydroxyphenethyl)- $N$-methyl-1,2,3,4-tetrahydroisoquinoline (S)-7f. 


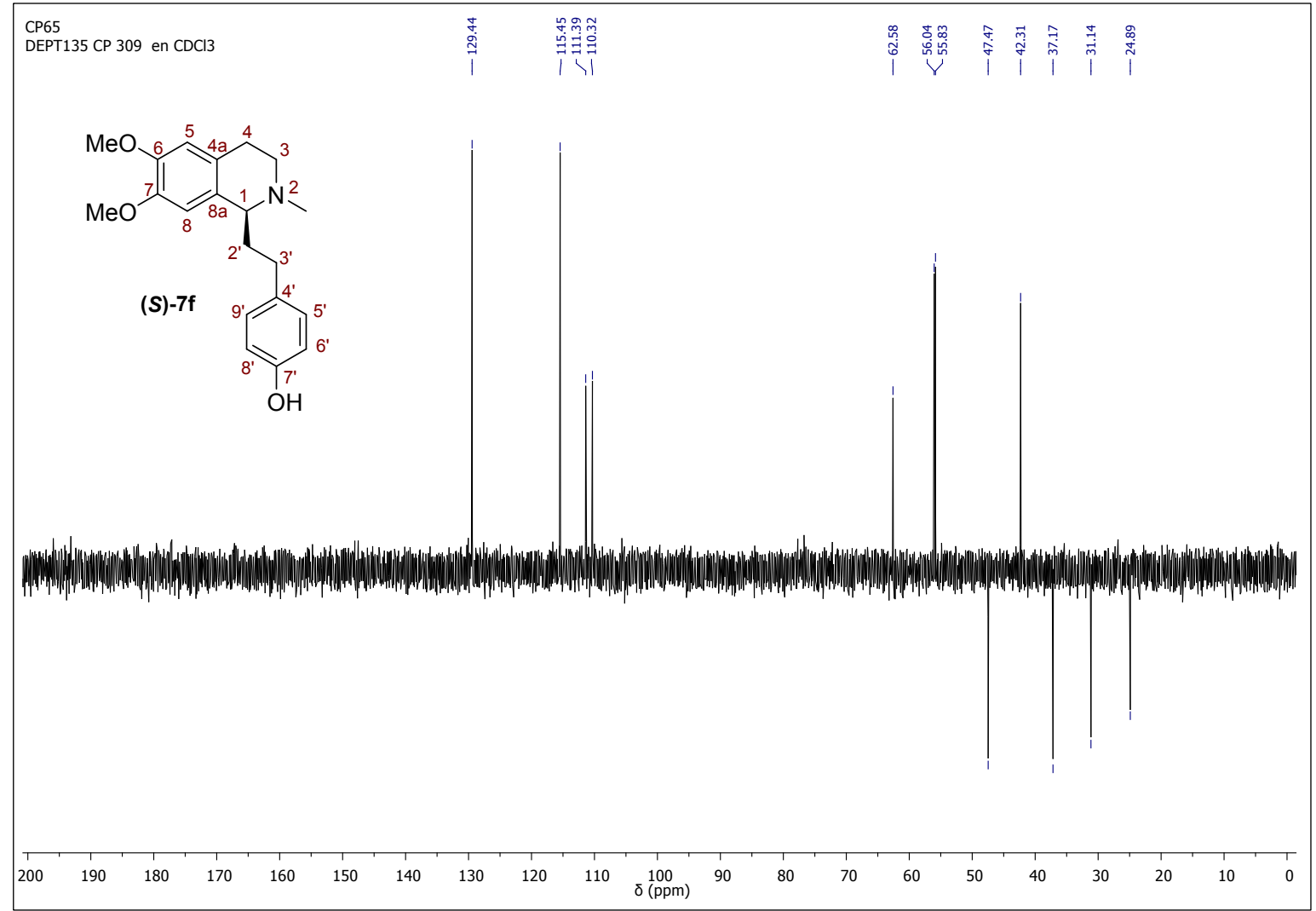

Figure S114. ${ }^{1} \mathrm{H}-\mathrm{NMR}$ spectrum of (S)-6,7-dimethoxy-1-(4-hydroxy-3-methoxyphenethyl)- $N$-methyl-1,2,3,4-tetrahydroisoquinoline (S)-7g

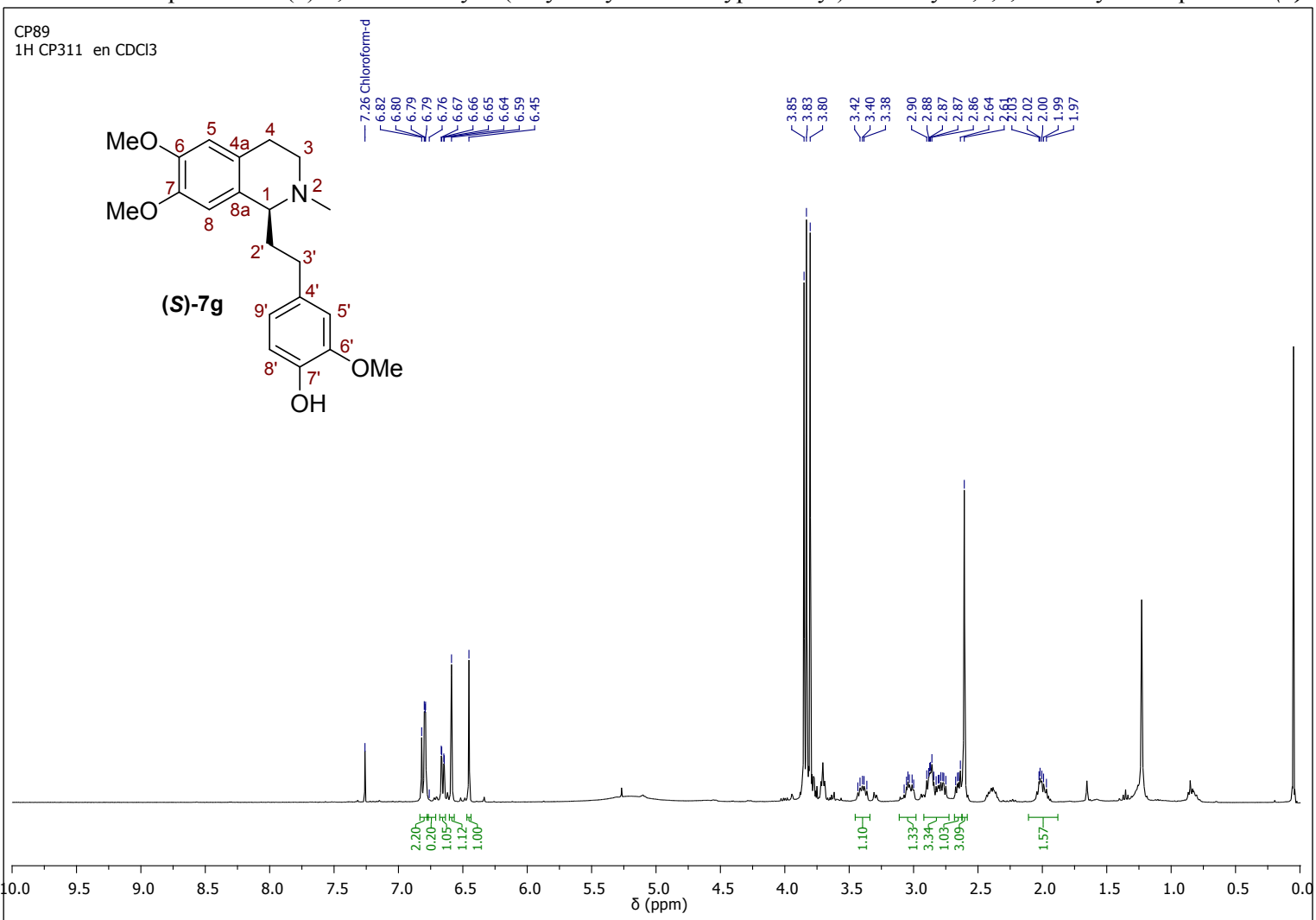


Figure S115. ${ }^{13} \mathrm{C}-\mathrm{NMR}$ spectrum of (S)-6,7-dimethoxy-1-(4-hydroxy-3-methoxyphenethyl)- $N$-methyl-1,2,3,4-tetrahydroisoquinoline (S)-7g

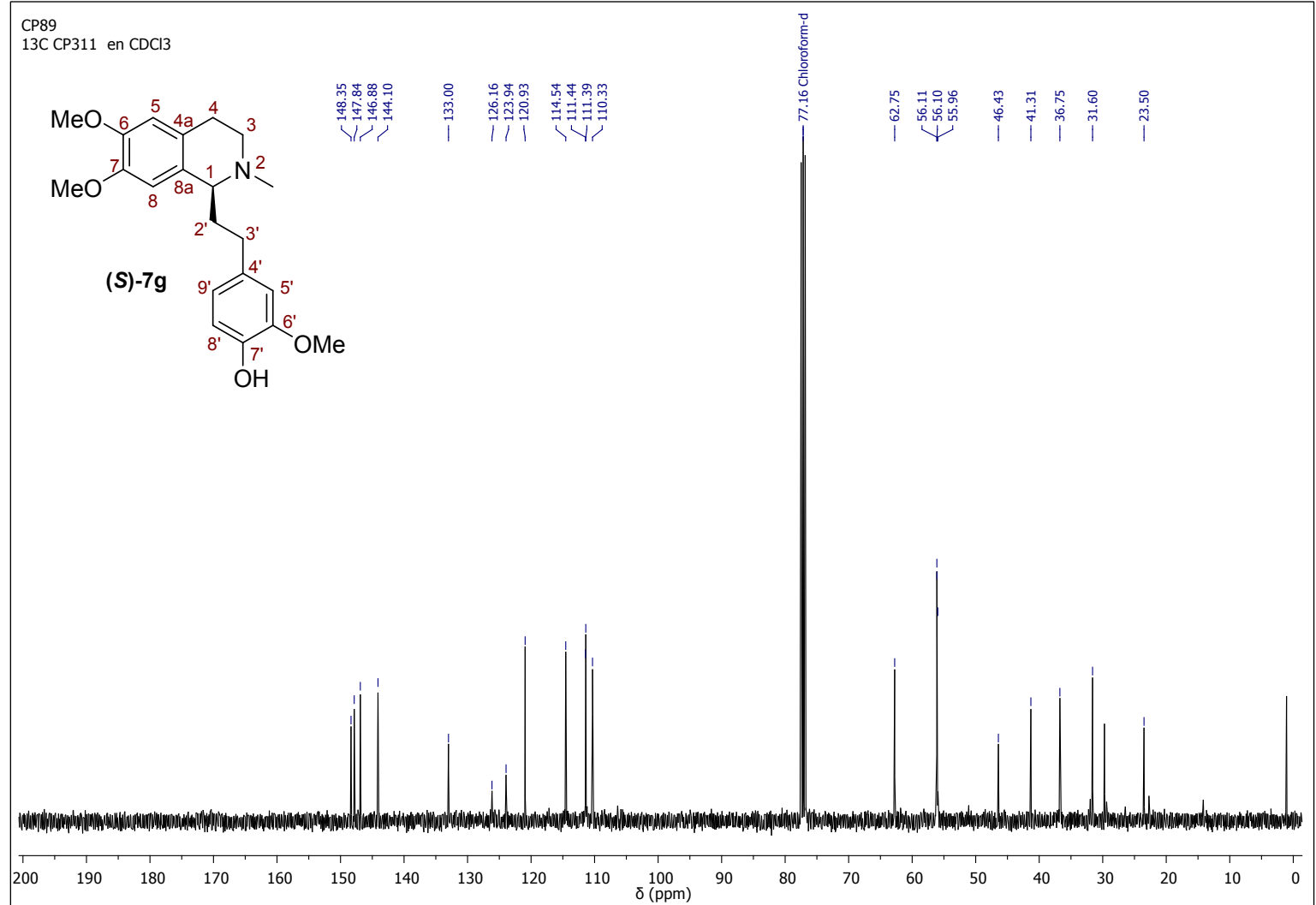

Figure S116. DEPT-135 spectrum of (S)-6,7-dimethoxy-1-(4-hydroxy-3-methoxyphenethyl)- $N$-methyl-1,2,3,4-tetrahydroisoquinoline (S)-7g

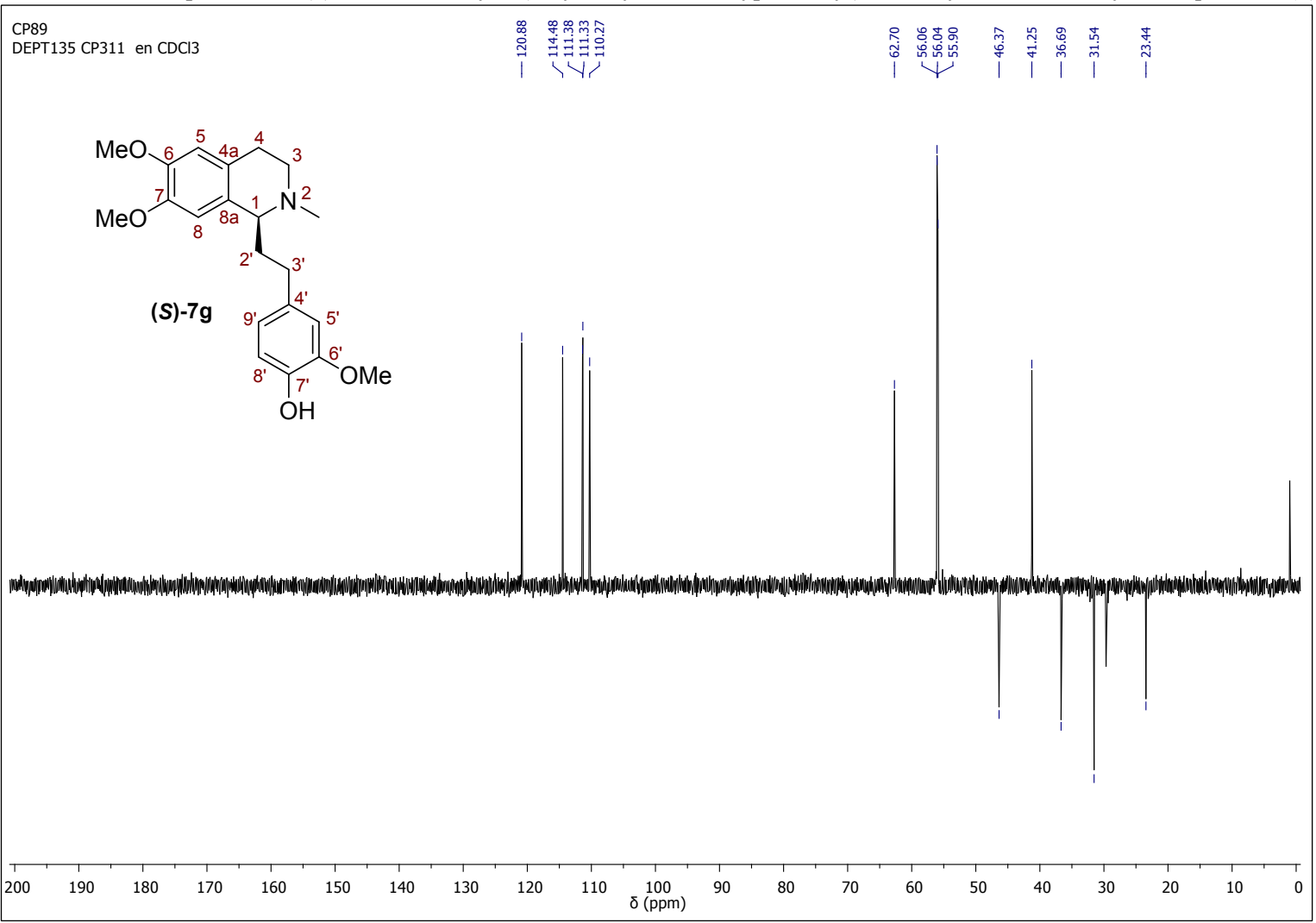


Figure S117. ${ }^{1} \mathrm{H}-\mathrm{NMR}$ spectrum of (S)-6,7-dimethoxy-1-(3-hydroxy-4-methoxyphenethyl)- $N$-methyl-1,2,3,4-tetrahydroisoquinoline (S)-7h .

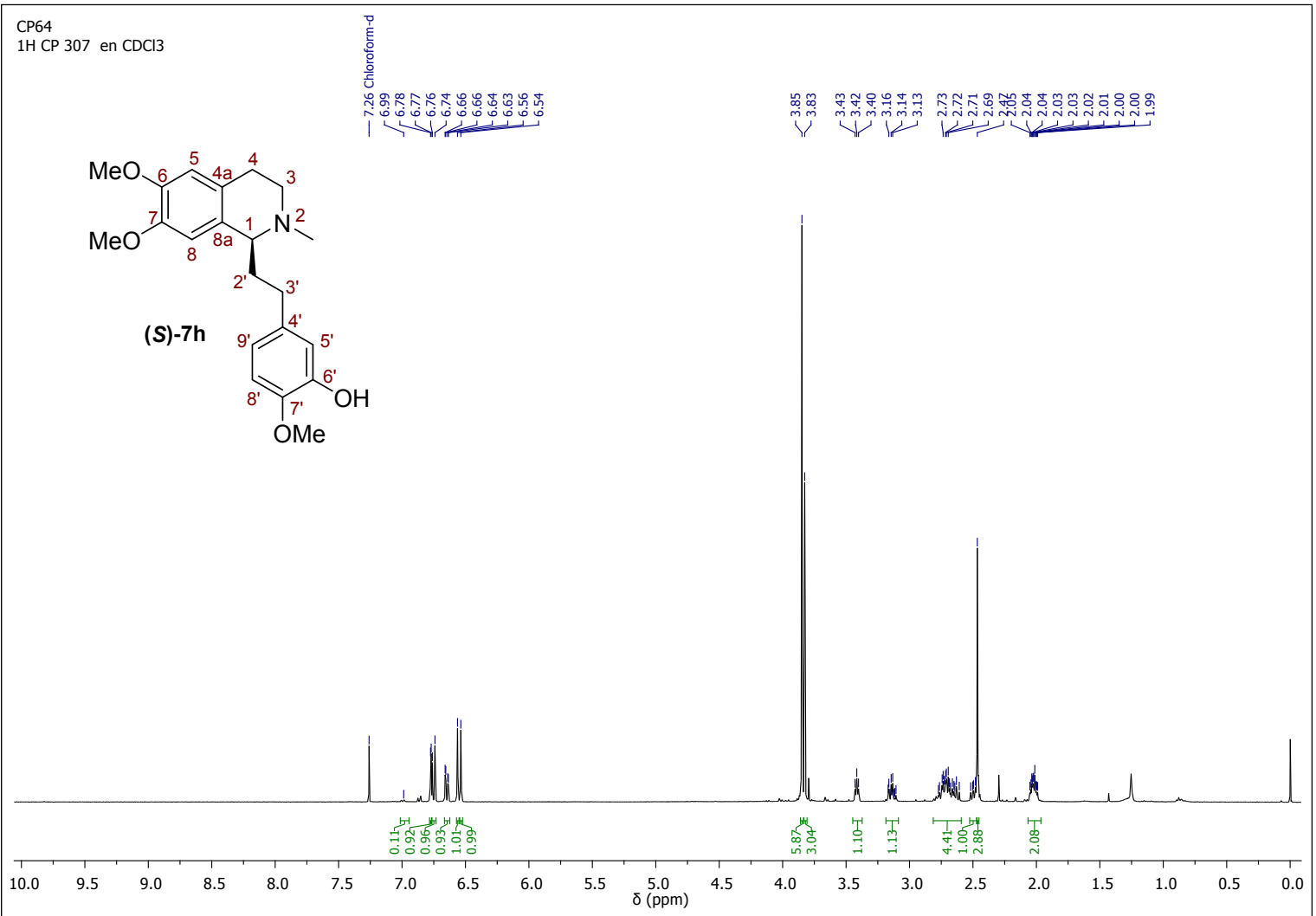

Figure S118. ${ }^{13} \mathrm{C}-\mathrm{NMR}$ spectrum of (S)-6,7-dimethoxy-1-(3-hydroxy-4-methoxyphenethyl)- $N$-methyl-1,2,3,4-tetrahydroisoquinoline (S)-7h

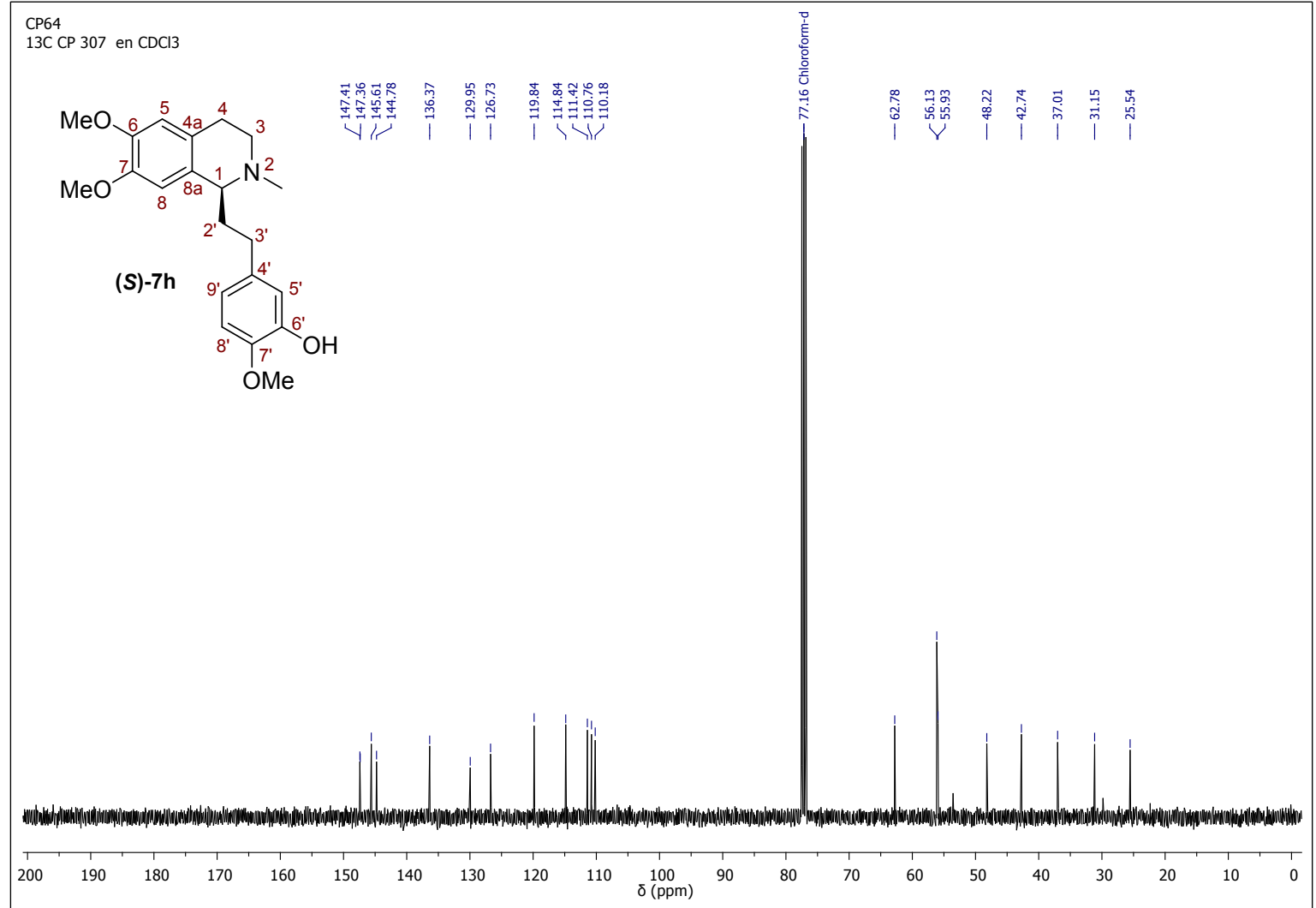


Figure S119. DEPT-135 spectrum of (S)-6,7-dimethoxy-1-(3-hydroxy-4-methoxyphenethyl)- $N$-methyl-1,2,3,4-tetrahydroisoquinoline (S)-7h

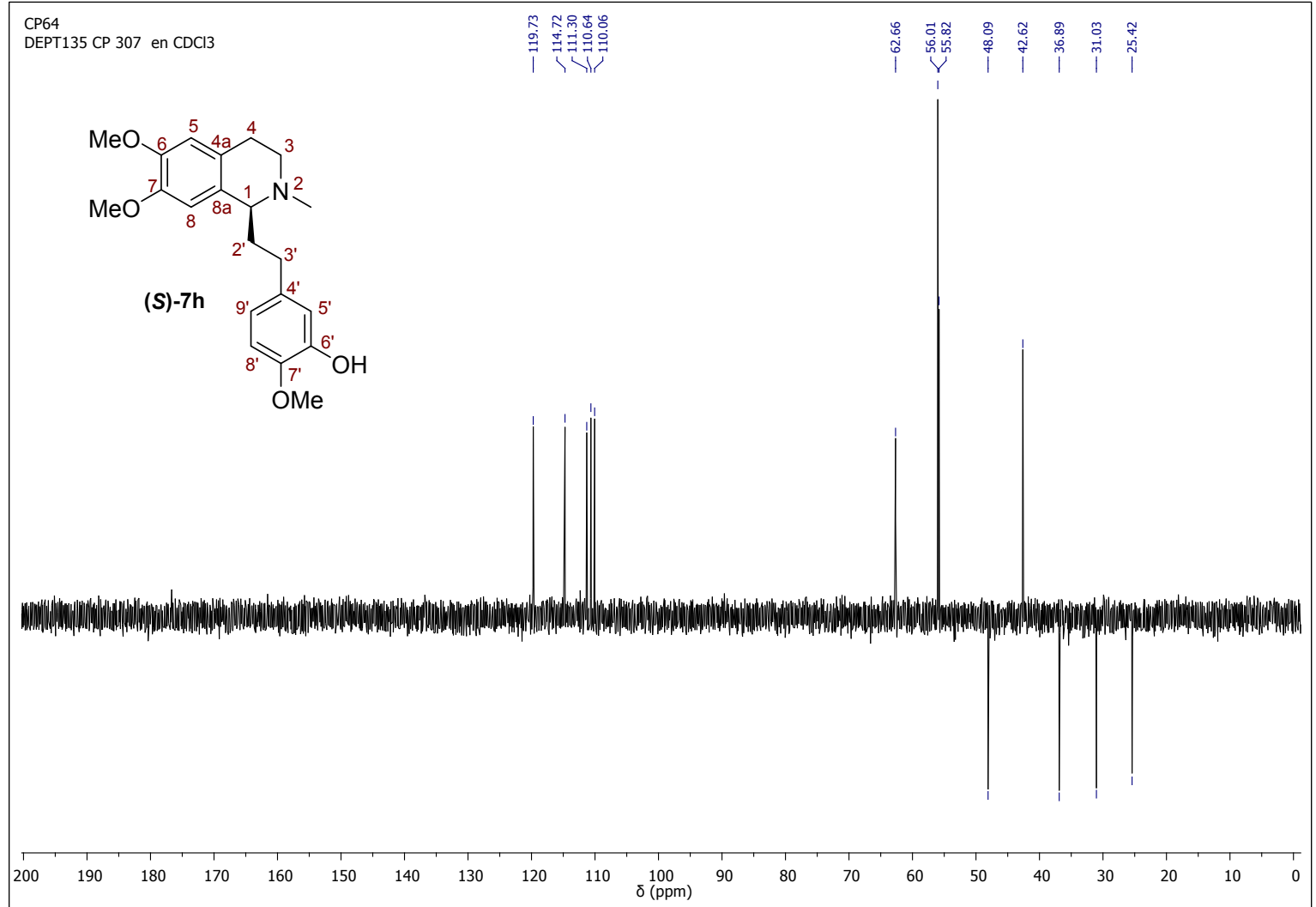

Figure S120. ${ }^{1}$ H-NMR spectrum of (S)-6,7-dimethoxy-1-(4-hydroxy-3,5-dimethoxyphenethyl)- $N$-methyl-1,2,3,4-tetrahydroisoquinoline (S)-7i.

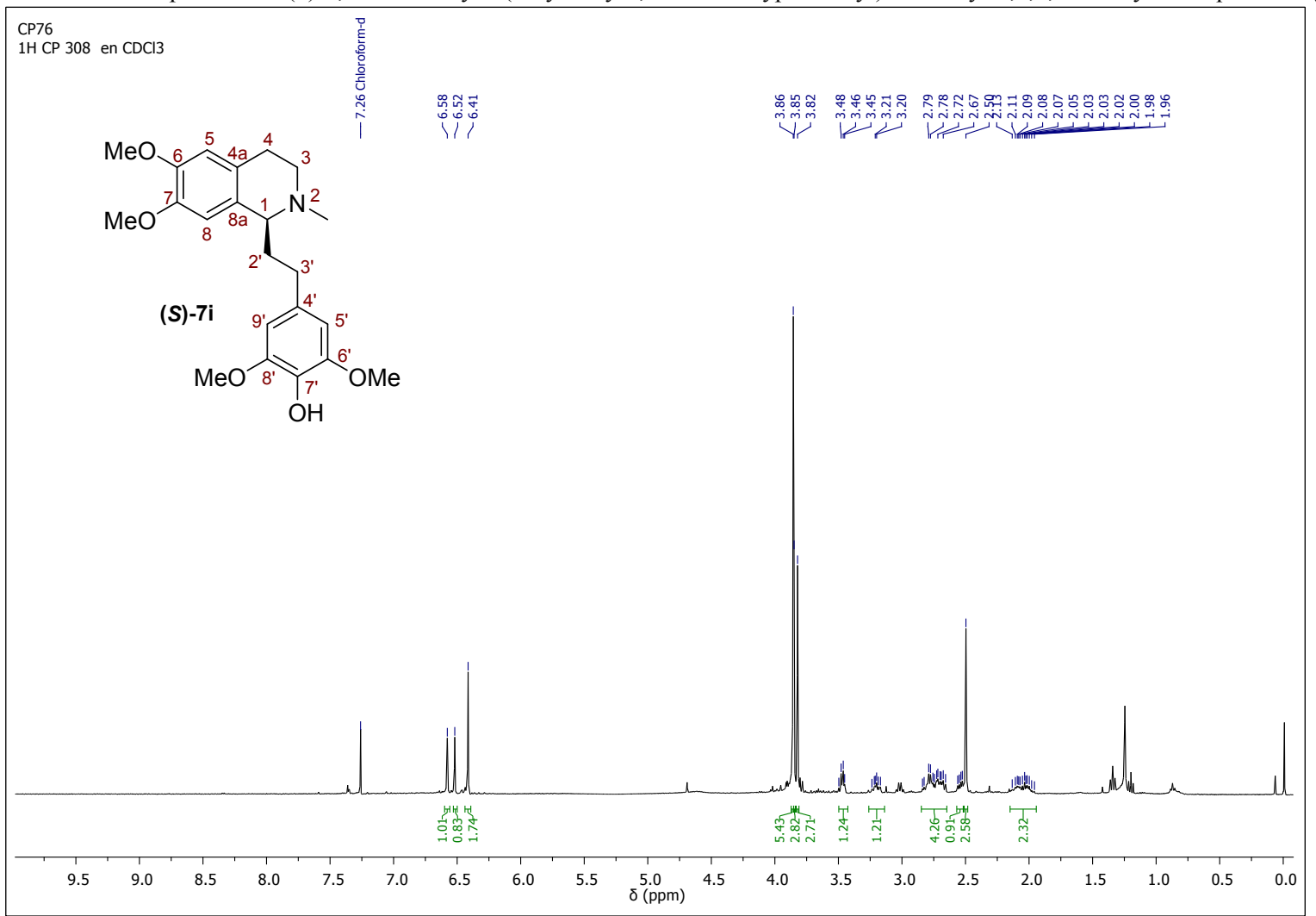


Figure S121. ${ }^{13} \mathrm{C}-\mathrm{NMR}$ spectrum of (S)-6,7-dimethoxy-1-(4-hydroxy-3,5-dimethoxyphenethyl)- $N$-methyl-1,2,3,4-tetrahydroisoquinoline (S)-7i.

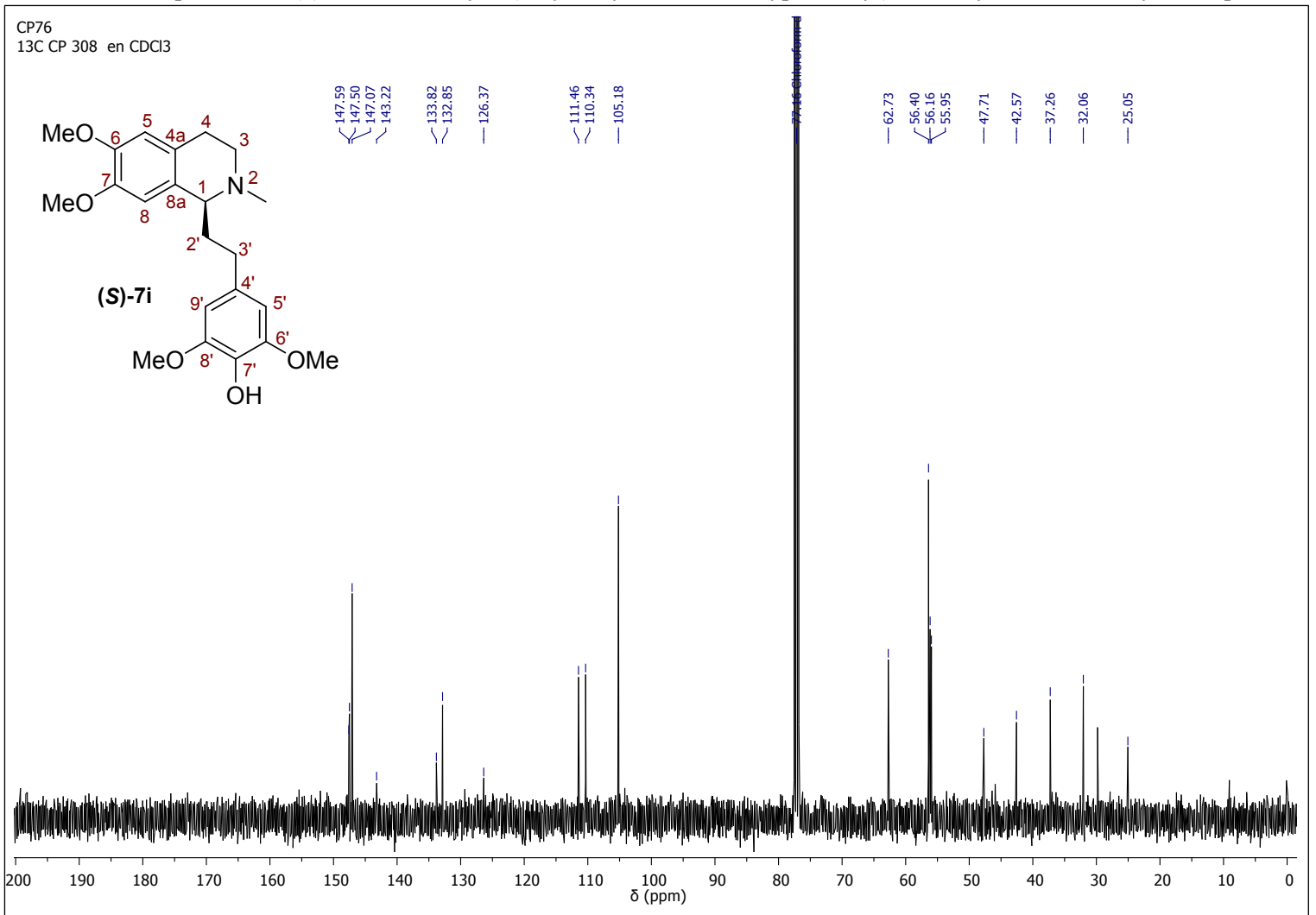

Figure S122. DEPT-135 spectrum of (S)-6,7-dimethoxy-1-(4-hydroxy-3,5-dimethoxyphenethyl)- $N$-methyl-1,2,3,4-tetrahydroisoquinoline (S)-7i.

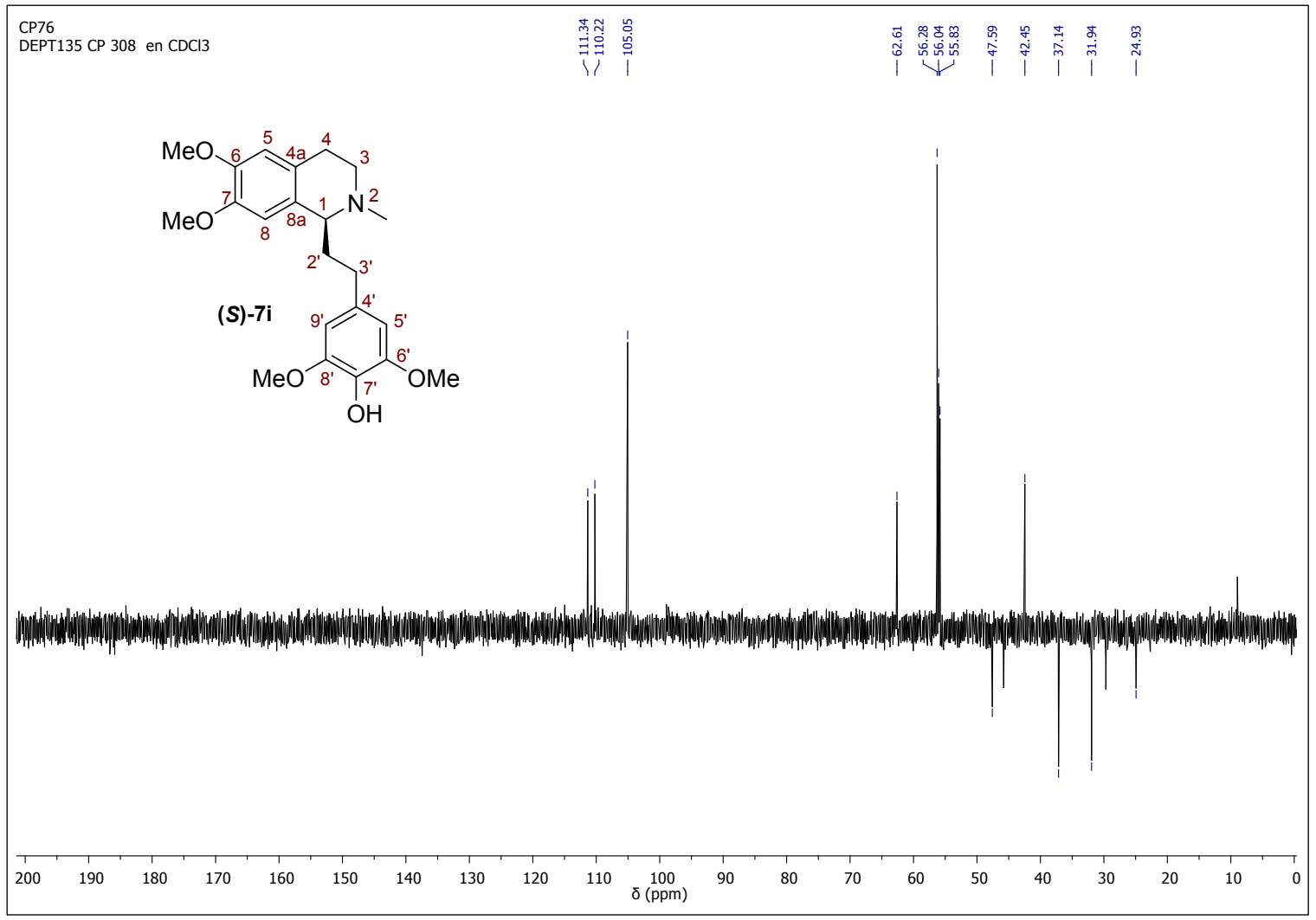




\section{Copies of chiral HPLC Chromatograms of (R)-7a-i and (S)-7a-i Dysoxylum alkaloids}

Figure S123. Chiral HPLC chromatogram of (R)-6,7-dimethoxy- $N$-methyl-1-phenethyl-1,2,3,4-tetrahydroisoquinoline (R)-7a

\begin{tabular}{cccccccc} 
Sample & Peak & $\begin{array}{c}\text { RT } \\
{[\mathrm{min}]}\end{array}$ & $\begin{array}{c}\text { Peak Height } \\
{[\mathrm{mAU}]}\end{array}$ & $\begin{array}{c}\text { Integral } \\
{[\%]}\end{array}$ & $\begin{array}{c}\text { Peak Area } \\
{[\mathrm{mAU} \text { s }]}\end{array}$ & $\begin{array}{c}\text { FWHM } \\
{[\mathrm{min}]}\end{array}$ & $\begin{array}{c}\text { Integral Region } \\
{[\mathrm{min}]}\end{array}$ \\
\hline & 1 & 6.06 & 2.016 & 2.58 & 11599.59 & 0.262 & $5.97-6.36$ \\
CP232 & 2 & 8.36 & 26.031 & 97.42 & 437996.74 & 0.396 & $8.16-8.97$ \\
\hline
\end{tabular}

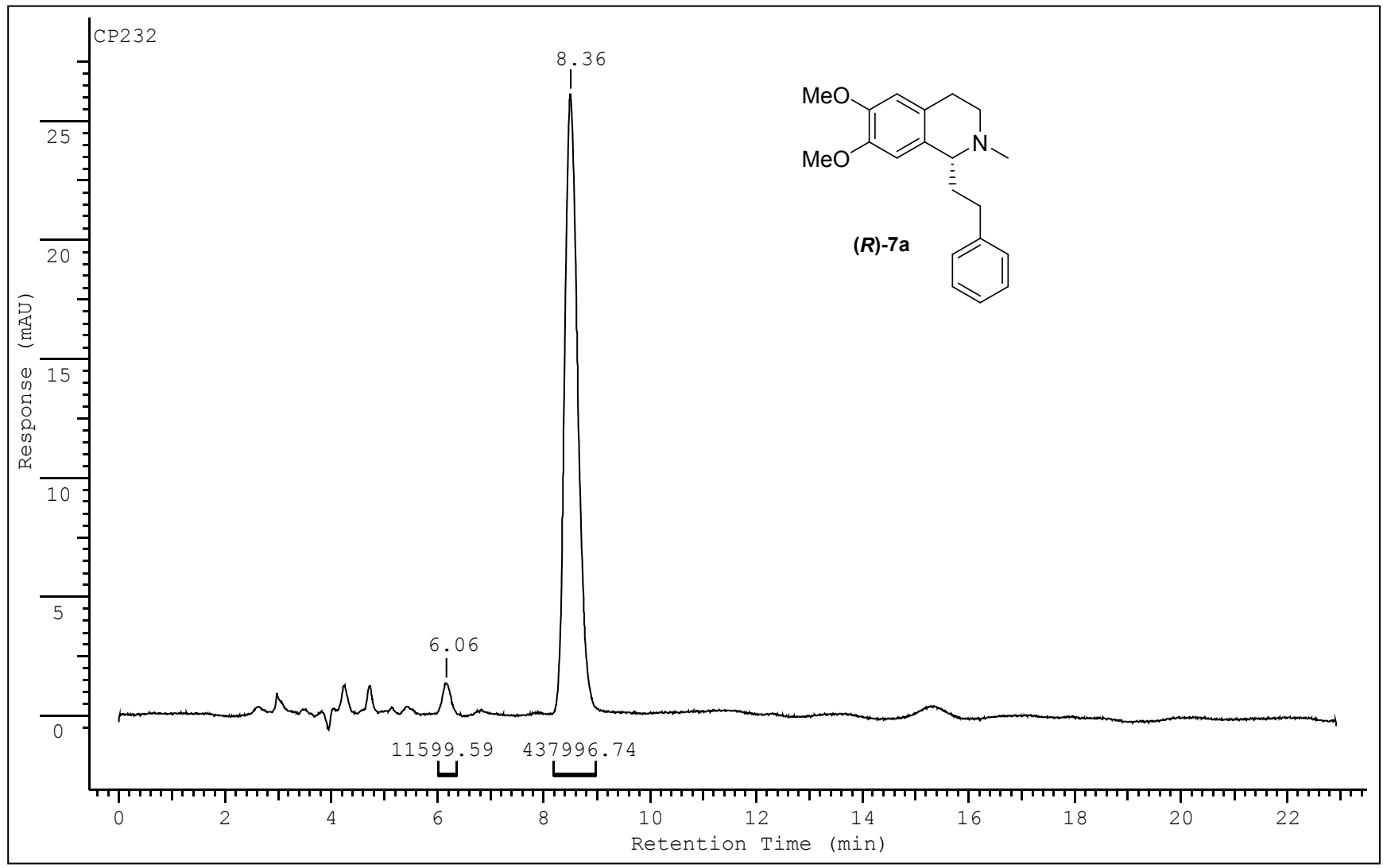


Figure S124. Chiral HPLC chromatogram of (R)-6,7-dimethoxy-1-(4-methoxyphenethyl)- $N$-methyl-1,2,3,4-tetrahydroisoquinoline (R)-7b

\begin{tabular}{ccccccccc} 
Sample & Peak & $\begin{array}{c}\text { RT } \\
{[\mathrm{min}]}\end{array}$ & $\begin{array}{c}\text { Peak Height } \\
{[\mathrm{mAU}]}\end{array}$ & $\begin{array}{c}\text { Integral } \\
{[\%]}\end{array}$ & $\begin{array}{c}\text { Peak Area } \\
{[\mathrm{mAU} \text { s }]}\end{array}$ & $\begin{array}{c}\text { FWHM } \\
{[\mathrm{min}]}\end{array}$ & $\begin{array}{c}\text { Integral Region } \\
{[\mathrm{min}]}\end{array}$ & $\begin{array}{c}\text { Anotation } \\
\text { CP301 }\end{array}$ \\
\hline & 1 & 6.88 & 0.248 & 4.70 & 133837.59 & 0.129 & $6.01-7.22$ \\
& 11.07 & 23.769 & 95.30 & 2713873.25 & 0.845 & $10.94-13.07$ \\
\hline
\end{tabular}

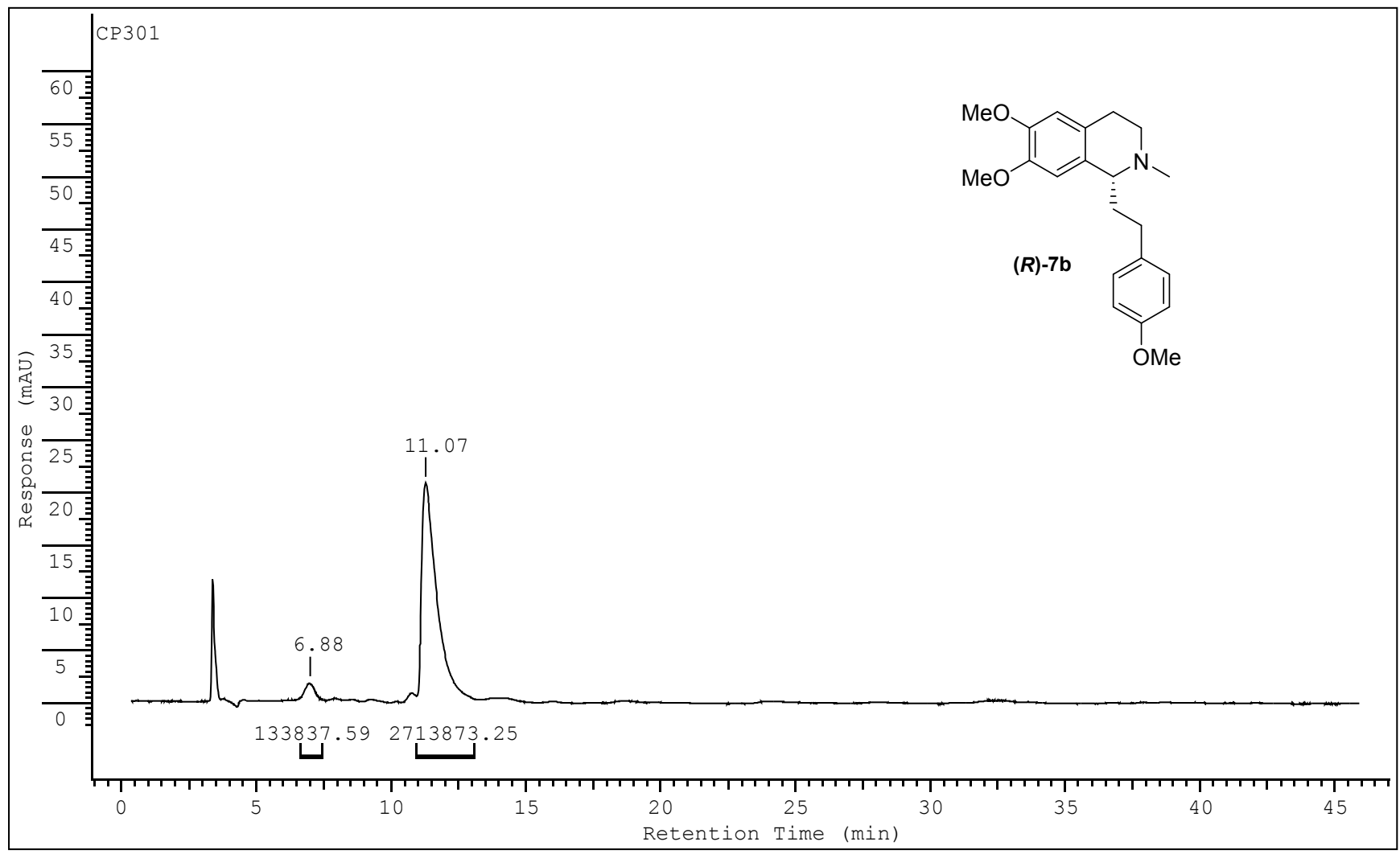


Figure S125. Chiral HPLC chromatogram of (R)-6,7-dimethoxy-1-(3,4-dimethoxyphenethyl)- $N$-methyl-1,2,3,4-tetrahydroisoquinoline (R)-7c

\begin{tabular}{cccccccc} 
Sample & Peak & $\begin{array}{c}\text { RT } \\
{[\mathrm{min}]}\end{array}$ & $\begin{array}{c}\text { Peak Height } \\
{[\mathrm{mAU}]}\end{array}$ & $\begin{array}{c}\text { Integral } \\
{[\%]}\end{array}$ & $\begin{array}{c}\text { Peak Area } \\
{[\mathrm{mAU} \text { s }]}\end{array}$ & $\begin{array}{c}\text { FWHM } \\
{[\mathrm{min}]}\end{array}$ & $\begin{array}{c}\text { Integral Region } \\
{[\mathrm{min}]}\end{array}$ \\
\hline & 1 & 13.35 & 12.428 & 4.40 & 442741.47 & 0.997 & $13.03-14.61$ \\
CP303 & 2 & 21.84 & 77.603 & 95.60 & 9626483.00 & 2.733 & $21.36-26.52$ \\
\hline
\end{tabular}

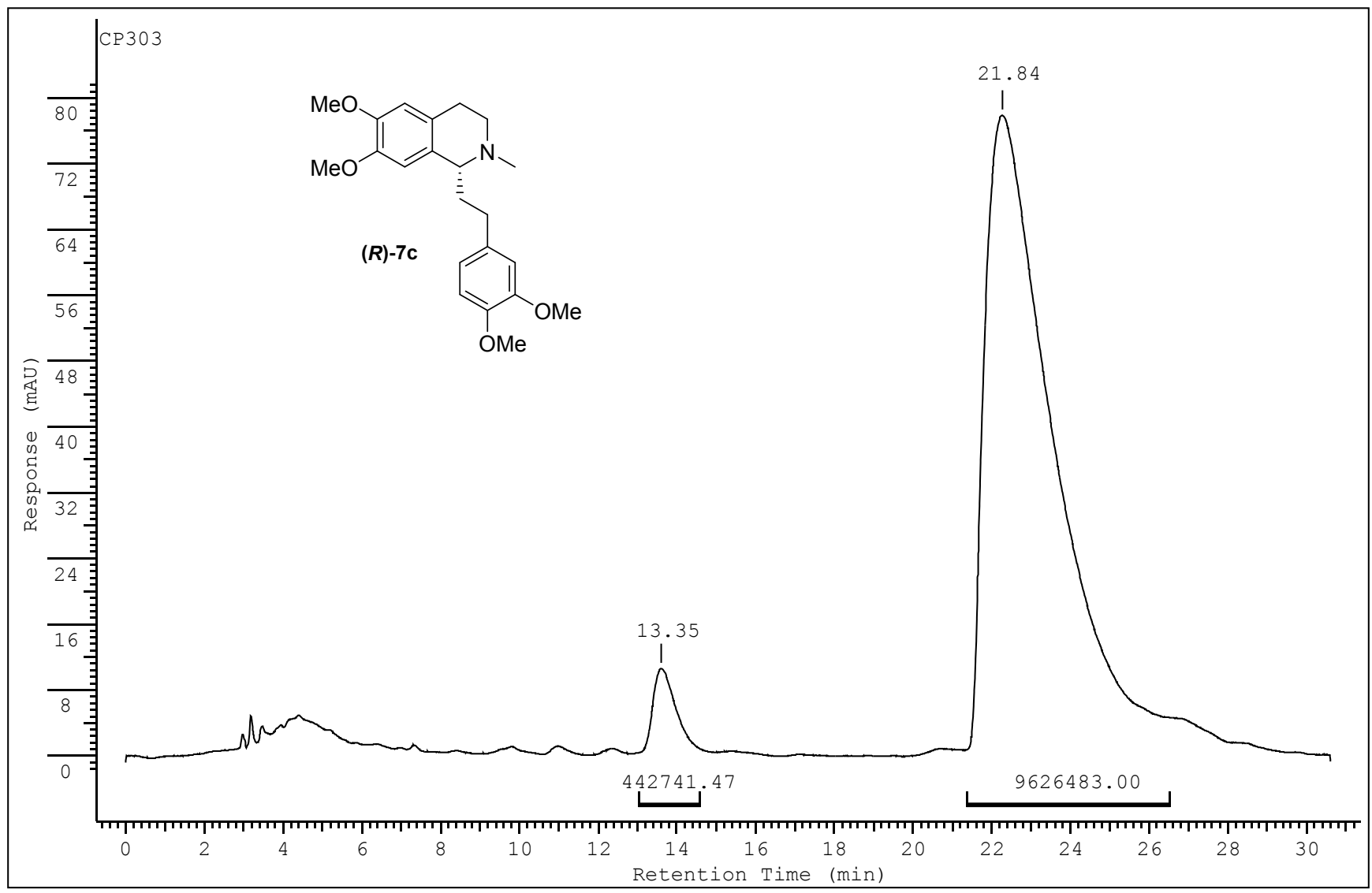


Figure S126. Chiral HPLC chromatogram of (R)-6,7-dimethoxy-1-(3,4,5-trimethoxyphenethyl)- $N$-methyl-1,2,3,4-tetrahydroisoquinoline (R)-7d

\begin{tabular}{cccccccc} 
Sample & Peak & $\begin{array}{c}\text { RT } \\
{[\mathrm{min}]}\end{array}$ & $\begin{array}{c}\text { Peak Height } \\
{[\mathrm{mAU}]}\end{array}$ & $\begin{array}{c}\text { Integral } \\
{[\%]}\end{array}$ & $\begin{array}{c}\text { Peak Area } \\
{[\mathrm{mAU} \text { s] }}\end{array}$ & $\begin{array}{c}\text { FWHM } \\
{[\mathrm{min}]}\end{array}$ & $\begin{array}{c}\text { Integral Region } \\
{[\mathrm{min}]}\end{array}$ \\
\hline & 1 & 12.48 & 1.187 & 5.93 & 115563.46 & 0.898 & $12.23-12.74$ \\
CP304 & 2 & 20.75 & 6.584 & 94.07 & 1833651.75 & 1.728 & $20.37-23.73$ \\
\hline
\end{tabular}

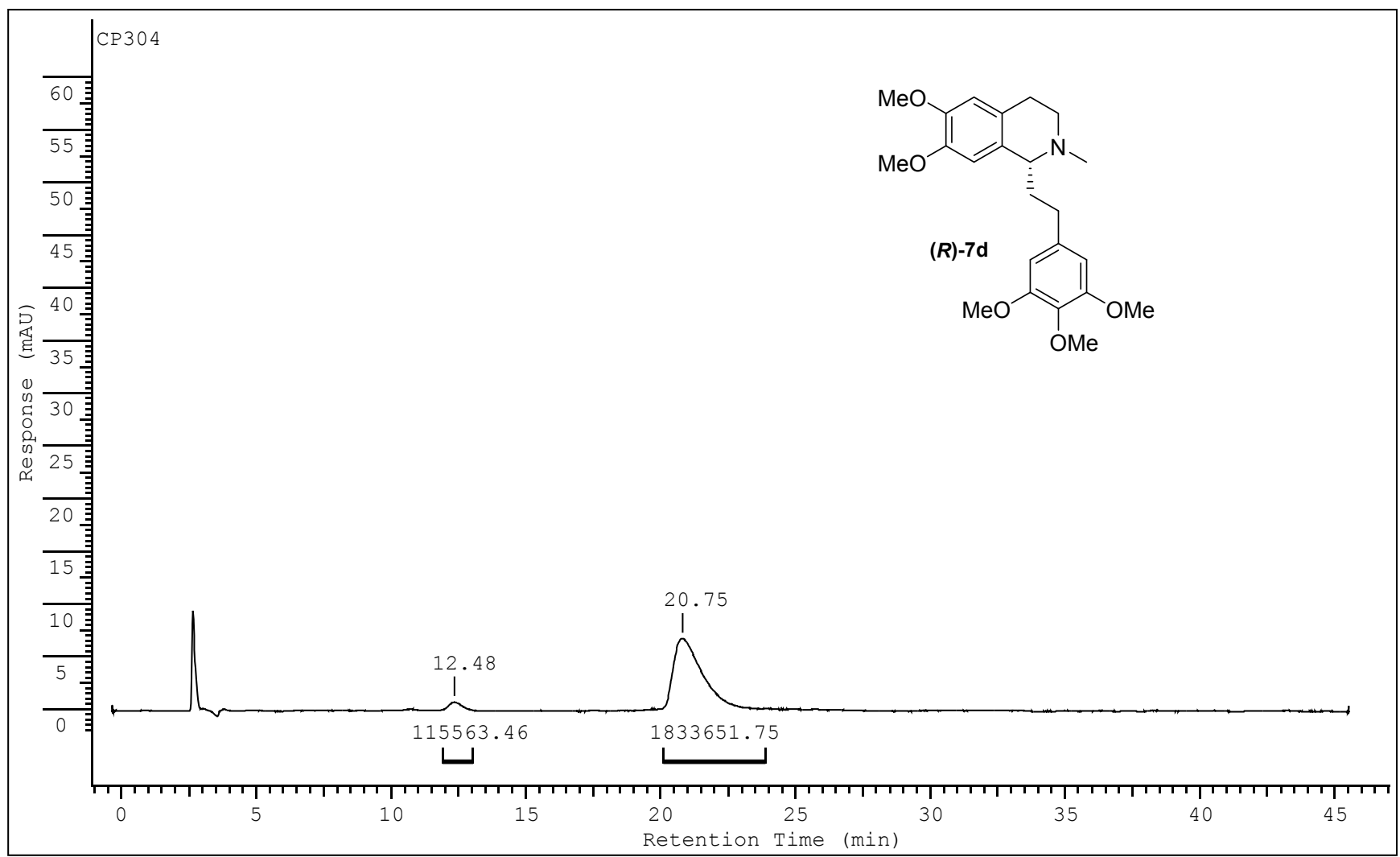


Figure S127. Chiral HPLC chromatogram of (R)-6,7-dimethoxy-1-(3,4-methylendioxyphenethyl)- $N$-methyl-1,2,3,4-tetrahydroisoquinoline (R)$7 e$

\begin{tabular}{cccccccc} 
Sample & Peak & $\begin{array}{c}\text { RT } \\
{[\mathrm{min}]}\end{array}$ & $\begin{array}{c}\text { Peak Height } \\
{[\mathrm{mAU}]}\end{array}$ & $\begin{array}{c}\text { Integral } \\
{[\%]}\end{array}$ & $\begin{array}{c}\text { Peak Area } \\
{[\mathrm{mAU} \text { s] }}\end{array}$ & $\begin{array}{c}\text { FWHM } \\
{[\mathrm{min}]}\end{array}$ & $\begin{array}{c}\text { Integral Region } \\
{[\mathrm{min}]}\end{array}$ \\
\hline & 1 & 7.69 & 42.938 & 6.39 & 831764.44 & 0.411 & $7.55-8.25$ \\
CP313 & 2 & 11.81 & 285.354 & 93.61 & 12182332.00 & 0.987 & $11.57-13.24$ \\
\hline
\end{tabular}

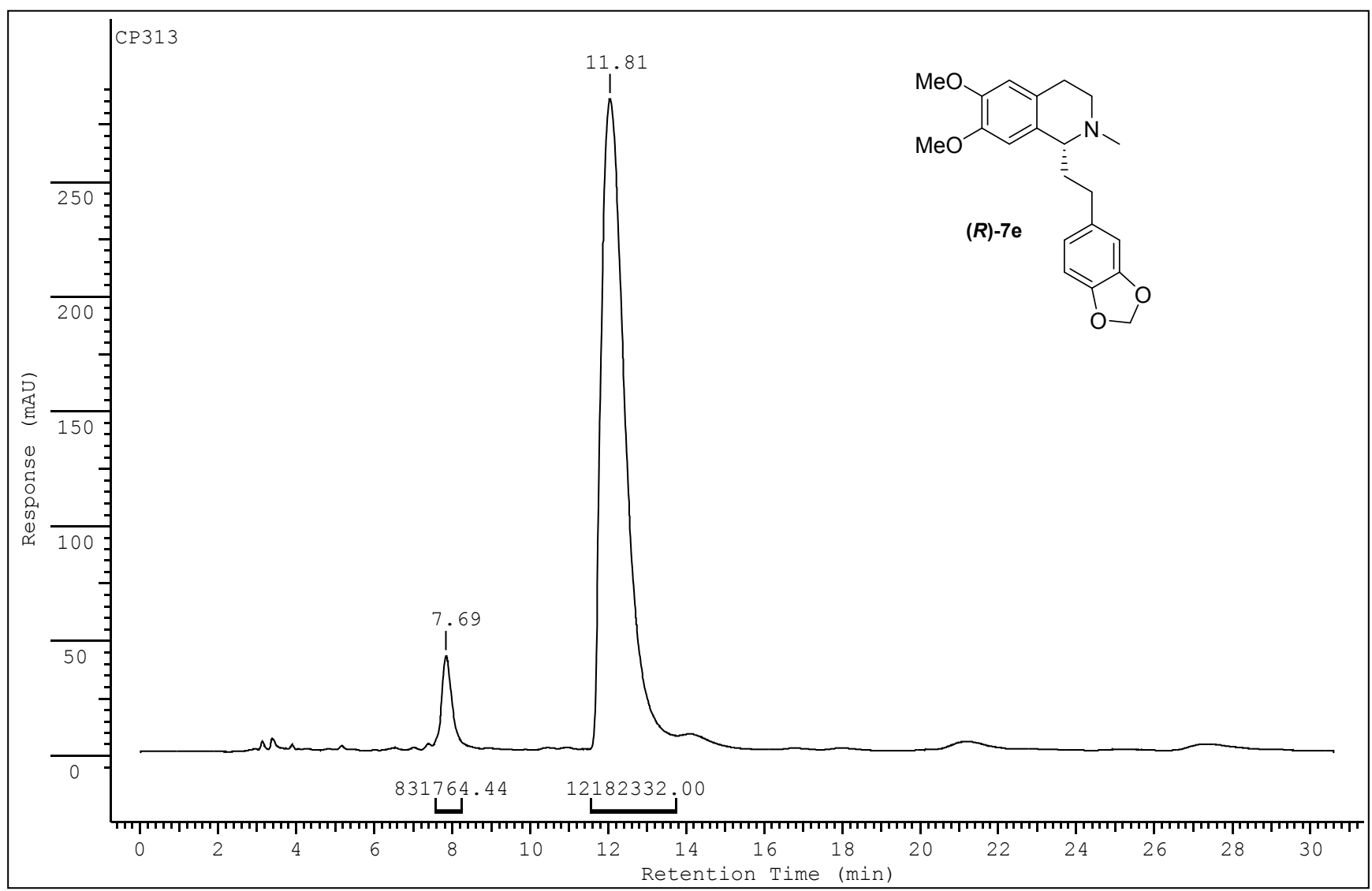


Figure S128. Chiral HPLC chromatogram of (R)-6,7-dimethoxy-1-(4-hydroxyphenethyl)- $N$-methyl-1,2,3,4-tetrahydroisoquinoline (R)-7f

\begin{tabular}{cccccccc} 
Sample & Peak & $\begin{array}{c}\text { RT } \\
{[\mathrm{min}]}\end{array}$ & $\begin{array}{c}\text { Peak Height } \\
{[\mathrm{mAU}]}\end{array}$ & $\begin{array}{c}\text { Integral } \\
{[\%]}\end{array}$ & $\begin{array}{c}\text { Peak Area } \\
{[\mathrm{mAU} \text { s }]}\end{array}$ & $\begin{array}{c}\text { FWHM } \\
{[\mathrm{min}]}\end{array}$ & $\begin{array}{c}\text { Integral Region } \\
{[\mathrm{min}]}\end{array}$ \\
\hline & 1 & 13.13 & 2.263 & 5.22 & 50056.43 & 1.451 & $12.83-13.84$ \\
CP305 & 2 & 32.37 & 6.704 & 94.78 & 909523.50 & 3.296 & $31.61-36.38$ \\
\hline
\end{tabular}

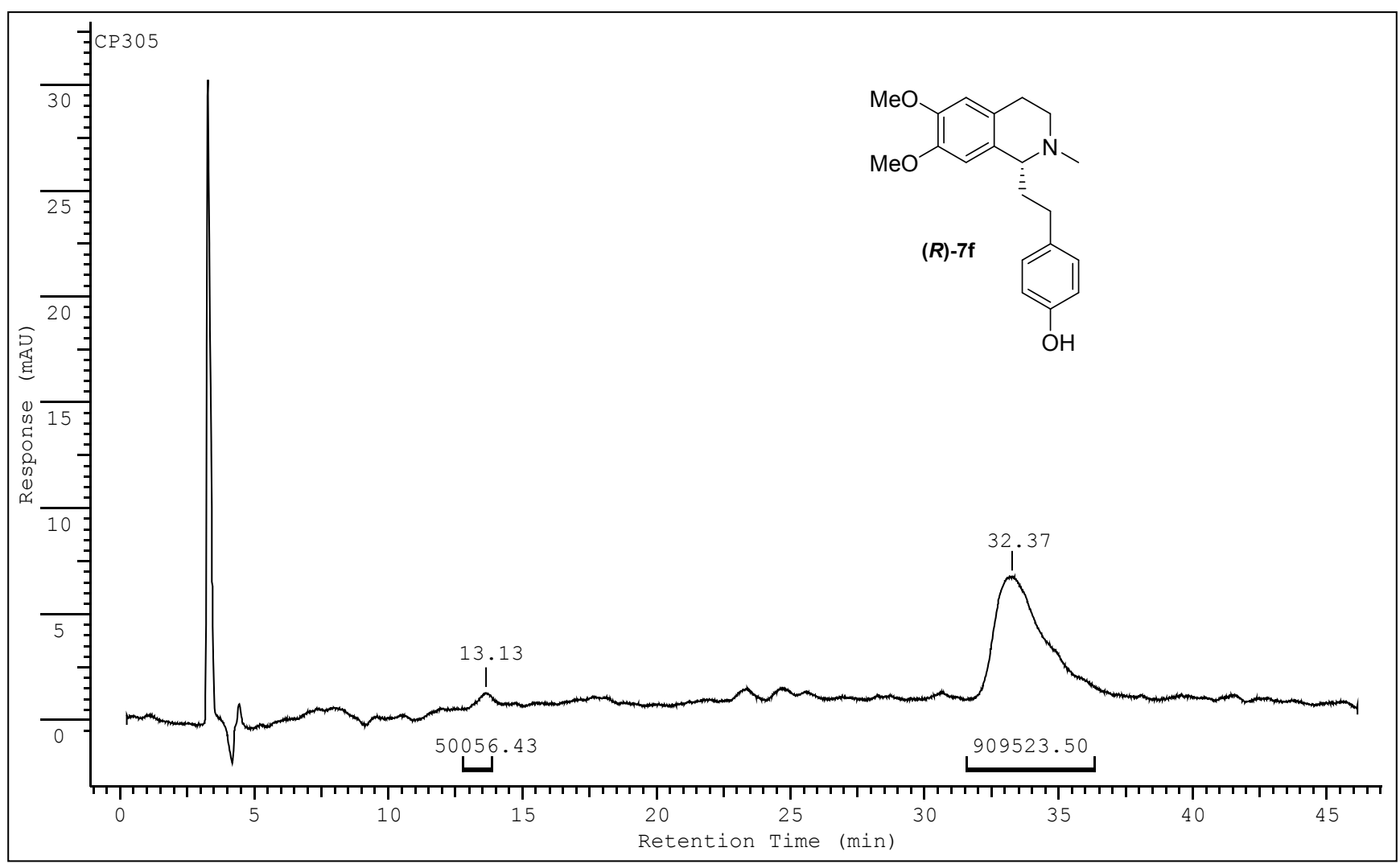


Figure S129. Chiral HPLC chromatogram of (R)-6,7-dimethoxy-1-(4-hydroxy-3-methoxyphenethyl)- $N$-methyl-1,2,3,4-tetrahydroisoquinoline (R)-7g

\begin{tabular}{cccccccc} 
Sample & Peak & $\begin{array}{c}\text { RT } \\
{[\mathrm{min}]}\end{array}$ & $\begin{array}{c}\text { Peak Height } \\
{[\mathrm{mAU}]}\end{array}$ & $\begin{array}{c}\text { Integral } \\
{[\%]}\end{array}$ & $\begin{array}{c}\text { Peak Area } \\
{[\mathrm{mAU} \text { s }]}\end{array}$ & $\begin{array}{c}\text { FWHM } \\
{[\mathrm{min}]}\end{array}$ & $\begin{array}{c}\text { Integral Region } \\
{[\mathrm{min}]}\end{array}$ \\
\hline & 1 & 16.70 & 3.957 & 5.55 & 181255.36 & 1.048 & $16.21-18.05$ \\
CP310 & 2 & 31.86 & 25.014 & 94.45 & 3085736.50 & 2.900 & $31.29-36.64$ \\
\hline
\end{tabular}

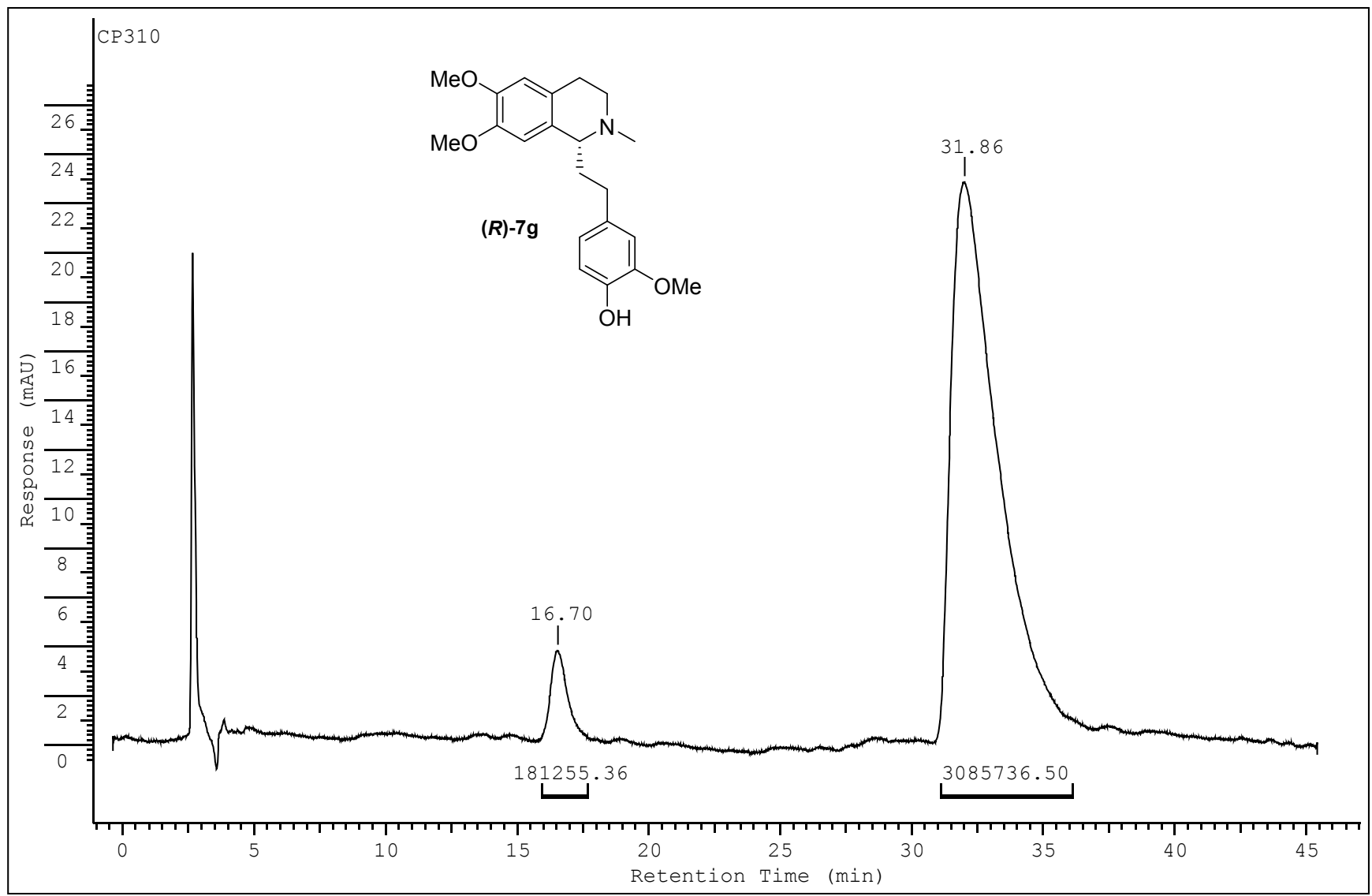


Figure S130. Chiral HPLC chromatogram of (R)-6,7-dimethoxy-1-(3-hydroxy-4-methoxyphenethyl)- $N$-methyl-1,2,3,4-tetrahydroisoquinoline (R)-7h

\begin{tabular}{cccccccc} 
Sample & Peak & $\begin{array}{c}\text { RT } \\
{[\mathrm{min}]}\end{array}$ & $\begin{array}{c}\text { Peak Height } \\
{[\mathrm{mAU}]}\end{array}$ & $\begin{array}{c}\text { Integral } \\
{[\%]}\end{array}$ & $\begin{array}{c}\text { Peak Area } \\
{[\mathrm{mAU} \text { s }]}\end{array}$ & $\begin{array}{c}\text { FWHM } \\
{[\mathrm{min}]}\end{array}$ & $\begin{array}{c}\text { Integral Region } \\
{[\mathrm{min}]}\end{array}$ \\
\hline & 1 & 17.06 & 8.034 & 5.69 & 319521.91 & 1.076 & $16.57-18.38$ \\
CP306 & 2 & 25.01 & 38.764 & 94.31 & 4961253.00 & 2.215 & $24.05-28.31$ \\
\hline
\end{tabular}

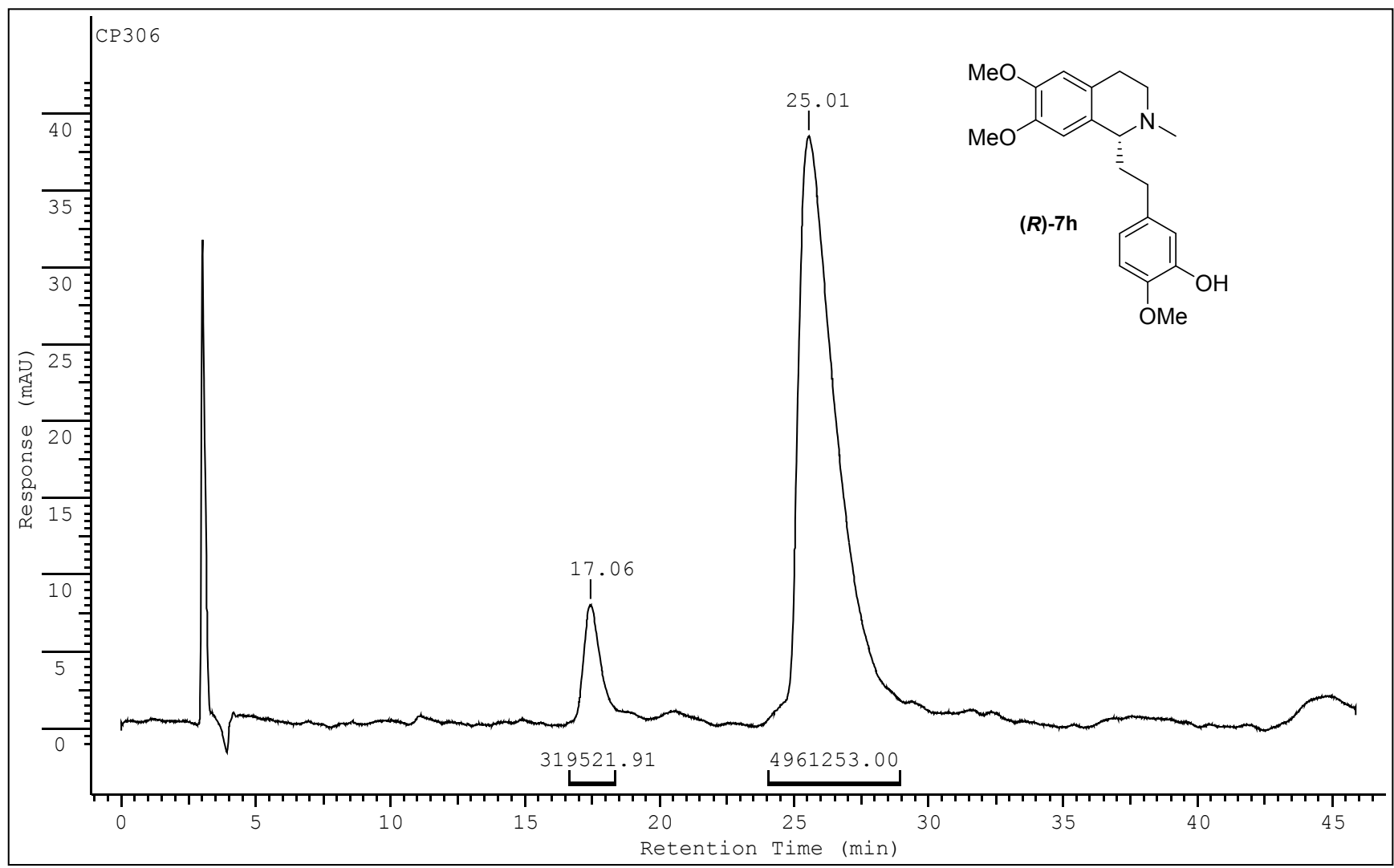


Figure S131. Chiral HPLC chromatogram of $(R)$-6,7-dimethoxy-1-(4-hydroxy-3,5-dimethoxyphenethyl)- $N$-methyl-1,2,3,4tetrahydroisoquinoline $(\boldsymbol{R})-\mathbf{7} \boldsymbol{i}$

\begin{tabular}{cccccccc} 
Sample & Peak & $\begin{array}{c}\text { RT } \\
{[\mathrm{min}]}\end{array}$ & $\begin{array}{c}\text { Peak Height } \\
{[\mathrm{mAU}]}\end{array}$ & $\begin{array}{c}\text { Integral } \\
{[\%]}\end{array}$ & $\begin{array}{c}\text { Peak Area } \\
{[\mathrm{mAU} \text { s }]}\end{array}$ & $\begin{array}{c}\text { FWHM } \\
{[\mathrm{min}]}\end{array}$ & $\begin{array}{c}\text { Integral Region } \\
{[\mathrm{min}]}\end{array}$ \\
\hline & 1 & 27.10 & 2.504 & 3.03 & 115471.95 & 8.483 & $26.71-29.11$ \\
CP312 & 2 & 36.48 & 27.204 & 96.97 & 3689808.25 & 3.080 & $35.79-41.37$ \\
\hline
\end{tabular}

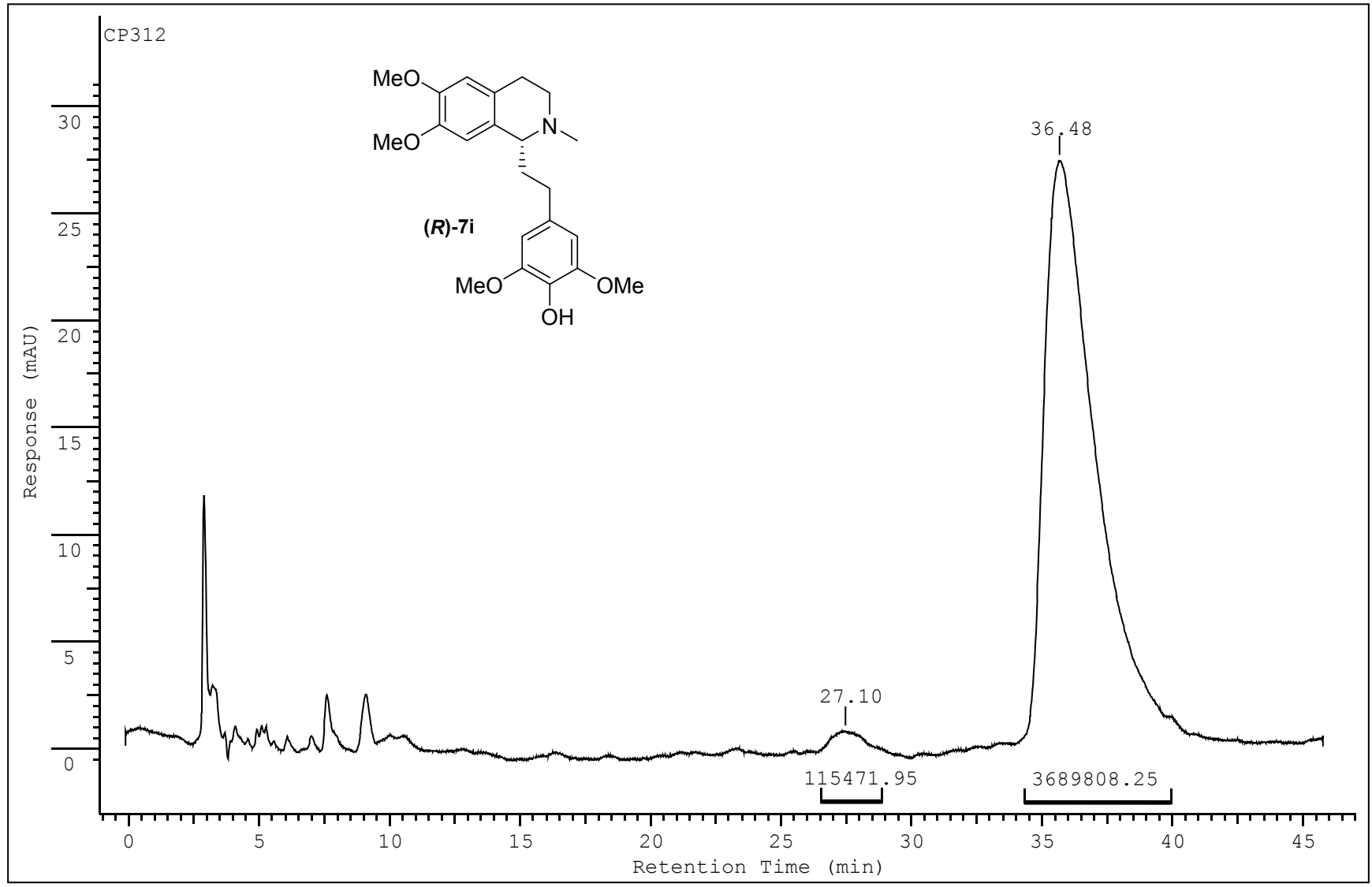


Figure S132. Chiral HPLC chromatogram of (S)-6,7-dimethoxy- $N$-methyl-1-phenethyl-1,2,3,4-tetrahydroisoquinoline (S)-7a

\begin{tabular}{cccccccc} 
Sample & Peak & $\begin{array}{c}\text { RT } \\
{[\mathrm{min}]}\end{array}$ & $\begin{array}{c}\text { Peak Height } \\
{[\mathrm{mAU}]}\end{array}$ & $\begin{array}{c}\text { Integral } \\
{[\%]}\end{array}$ & $\begin{array}{c}\text { Peak Area } \\
{[\mathrm{mAU} \text { s }]}\end{array}$ & $\begin{array}{c}\text { FWHM } \\
{[\mathrm{min}]}\end{array}$ & $\begin{array}{c}\text { Integral Region } \\
{[\mathrm{min}]}\end{array}$ \\
\hline & 1 & 5.97 & 57.850 & 96.53 & 631863.04 & 0.256 & $5.66-6.48$ \\
CP236 & 2 & 8.33 & 1.640 & 3.47 & 22713.81 & 0.383 & $8.18-8.74$ \\
\hline
\end{tabular}

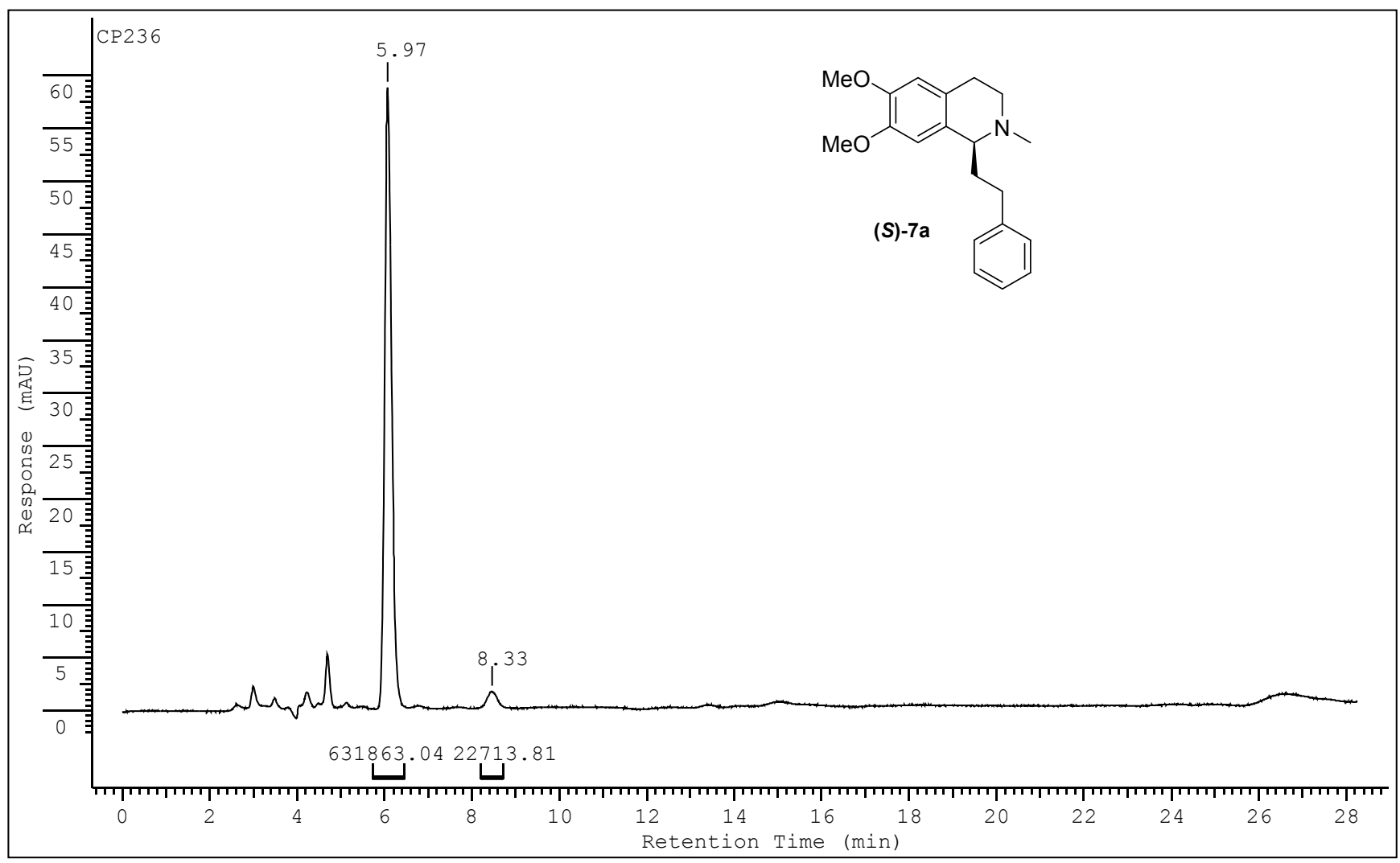


Figure S133. Chiral HPLC chromatogram of (S)-6,7-dimethoxy-1-(4-methoxyphenethyl)- $N$-methyl-1,2,3,4-tetrahydroisoquinoline ( $\boldsymbol{S}$ )-7b

\begin{tabular}{|c|c|c|c|c|c|c|c|c|}
\hline Sample & Peak & $\begin{array}{c}\mathrm{RT} \\
{[\mathrm{min}]}\end{array}$ & $\begin{array}{c}\text { Peak Height } \\
{[\mathrm{mAU}]}\end{array}$ & $\begin{array}{c}\text { Integral } \\
{[\%]}\end{array}$ & $\begin{array}{c}\text { Peak Area } \\
{\left[\mathrm{mAU}^{\star} \mathrm{s}\right]}\end{array}$ & $\begin{array}{l}\text { EWHM } \\
\text { [min] }\end{array}$ & $\begin{array}{c}\text { Integral Region } \\
\text { [min] }\end{array}$ & Anotation \\
\hline & 1 & 6.45 & 476.199 & 95.85 & 8675240.00 & 0.381 & $6.31-7.34$ & \\
\hline CP275 & 2 & 11.05 & 16.529 & 4.15 & 375421.84 & 0.837 & $10.97-11.67$ & \\
\hline
\end{tabular}

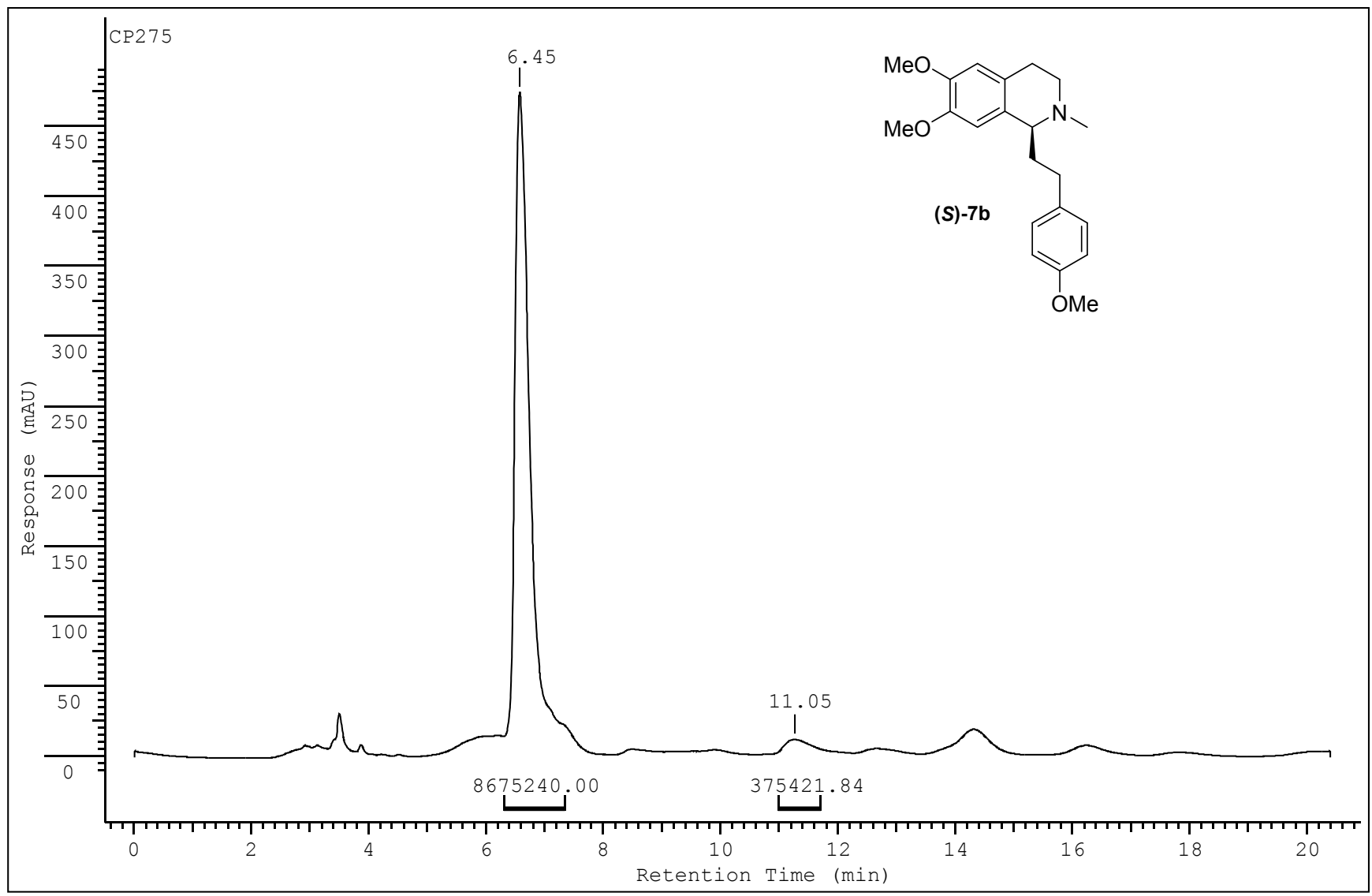


Figure S134. Chiral HPLC chromatogram of (S)-6,7-dimethoxy-1-(3,4-dimethoxyphenethyl)- $N$-methyl-1,2,3,4-tetrahydroisoquinoline (S)-7c

\begin{tabular}{cccccccc} 
Sample & Peak & $\begin{array}{c}\text { RT } \\
{[\mathrm{min}]}\end{array}$ & $\begin{array}{c}\text { Peak Height } \\
{[\mathrm{mAU}]}\end{array}$ & $\begin{array}{c}\text { Integral } \\
{[\%]}\end{array}$ & $\begin{array}{c}\text { Peak Area } \\
{[\mathrm{mAU} \text { s }]}\end{array}$ & $\begin{array}{c}\text { FWHM } \\
{[\mathrm{min}]}\end{array}$ & $\begin{array}{c}\text { Integral Region } \\
{[\mathrm{min}]}\end{array}$ \\
\hline & 1 & 12.51 & 143.011 & 95.42 & 8606440.00 & 1.304 & $12.07-15.98$ \\
CP302 & 2 & 23.52 & 7.27 & 4.58 & 413089.44 & 1.809 & $23.16-25.75$ \\
\hline
\end{tabular}

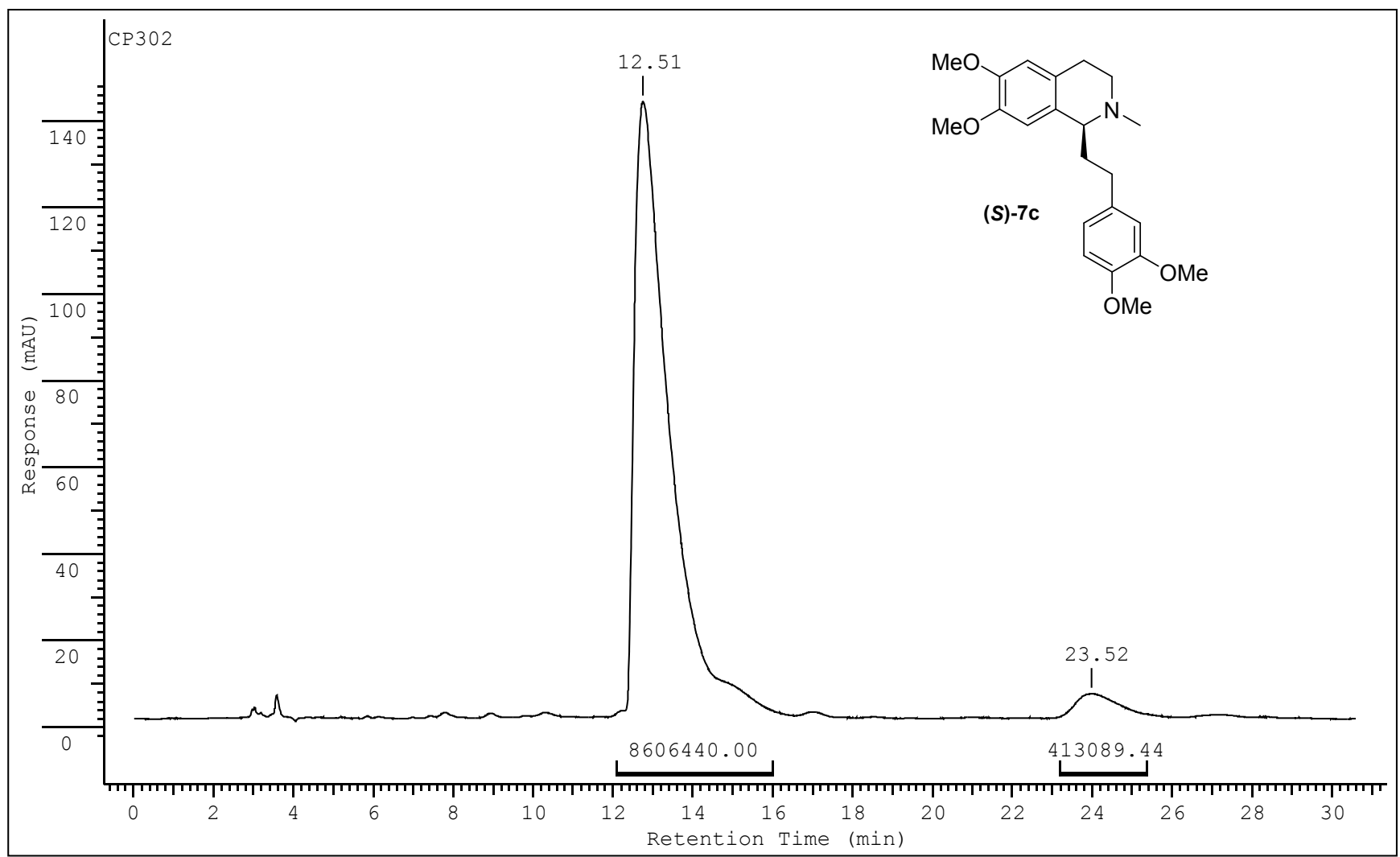


Figure S135. Chiral HPLC chromatogram of (S)-6,7-dimethoxy-1-(3,4,5-trimethoxyphenethyl)- $N$-methyl-1,2,3,4-tetrahydroisoquinoline (S)-7d

\begin{tabular}{|c|c|c|c|c|c|c|c|c|}
\hline Sample & Peak & $\begin{array}{c}\mathrm{RT} \\
{[\mathrm{min}]}\end{array}$ & $\begin{array}{l}\text { Peak Height } \\
{[\mathrm{mAU}]}\end{array}$ & $\begin{array}{c}\text { Integral } \\
{[\%]}\end{array}$ & $\begin{array}{l}\text { Peak Area } \\
{\left[\mathrm{mAU}^{\star} \mathrm{s}\right]}\end{array}$ & $\begin{array}{l}\text { FWHM } \\
{[\mathrm{min}]}\end{array}$ & $\begin{array}{c}\text { Integral Region } \\
\text { [min] }\end{array}$ & Anotation \\
\hline & 1 & 13.07 & 215.735 & 97.04 & 9250636.00 & 1.022 & $12.88-13.71$ & \\
\hline CP276 & 2 & 21.18 & 13.743 & 2.96 & 282589.69 & 1.489 & $20.12-21.39$ & \\
\hline
\end{tabular}

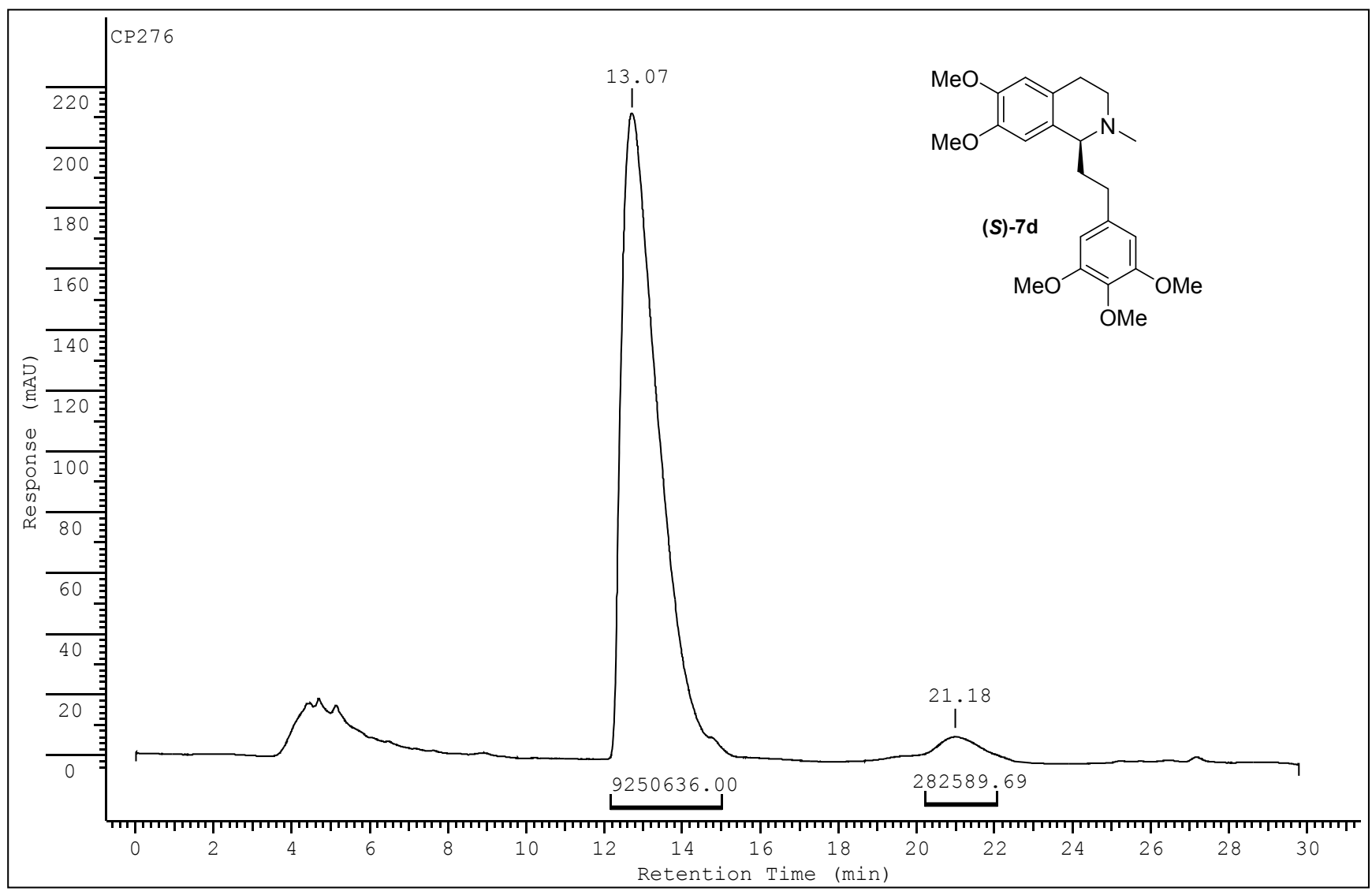


Figure S136. Chiral HPLC chromatogram of (S)-6,7-dimethoxy-1-(3,4-methylendioxyphenethyl)- $N$-methyl-1,2,3,4-tetrahydroisoquinoline (S)$7 e$

\begin{tabular}{|c|c|c|c|c|c|c|c|c|}
\hline Sample & Peak & $\begin{array}{c}\mathrm{RT} \\
{[\mathrm{min}]}\end{array}$ & $\begin{array}{l}\text { Peak Height } \\
{[\mathrm{mAU}]}\end{array}$ & $\begin{array}{c}\text { Integral } \\
{[\%]}\end{array}$ & $\begin{array}{l}\text { Peak Area } \\
{\left[\mathrm{mAU}^{\star} \mathrm{s}\right]}\end{array}$ & $\begin{array}{l}\text { FWHM } \\
{[\mathrm{min}]}\end{array}$ & $\begin{array}{c}\text { Integral Region } \\
\text { [min] }\end{array}$ & Anotation \\
\hline & 1 & 7.83 & 12.086 & 96.69 & 182960.81 & 0.358 & $7.59-8.49$ & \\
\hline CP2 277 & 2 & 12.85 & 0.482 & 3.31 & 6264.90 & 1.596 & $12.55-13.11$ & \\
\hline
\end{tabular}

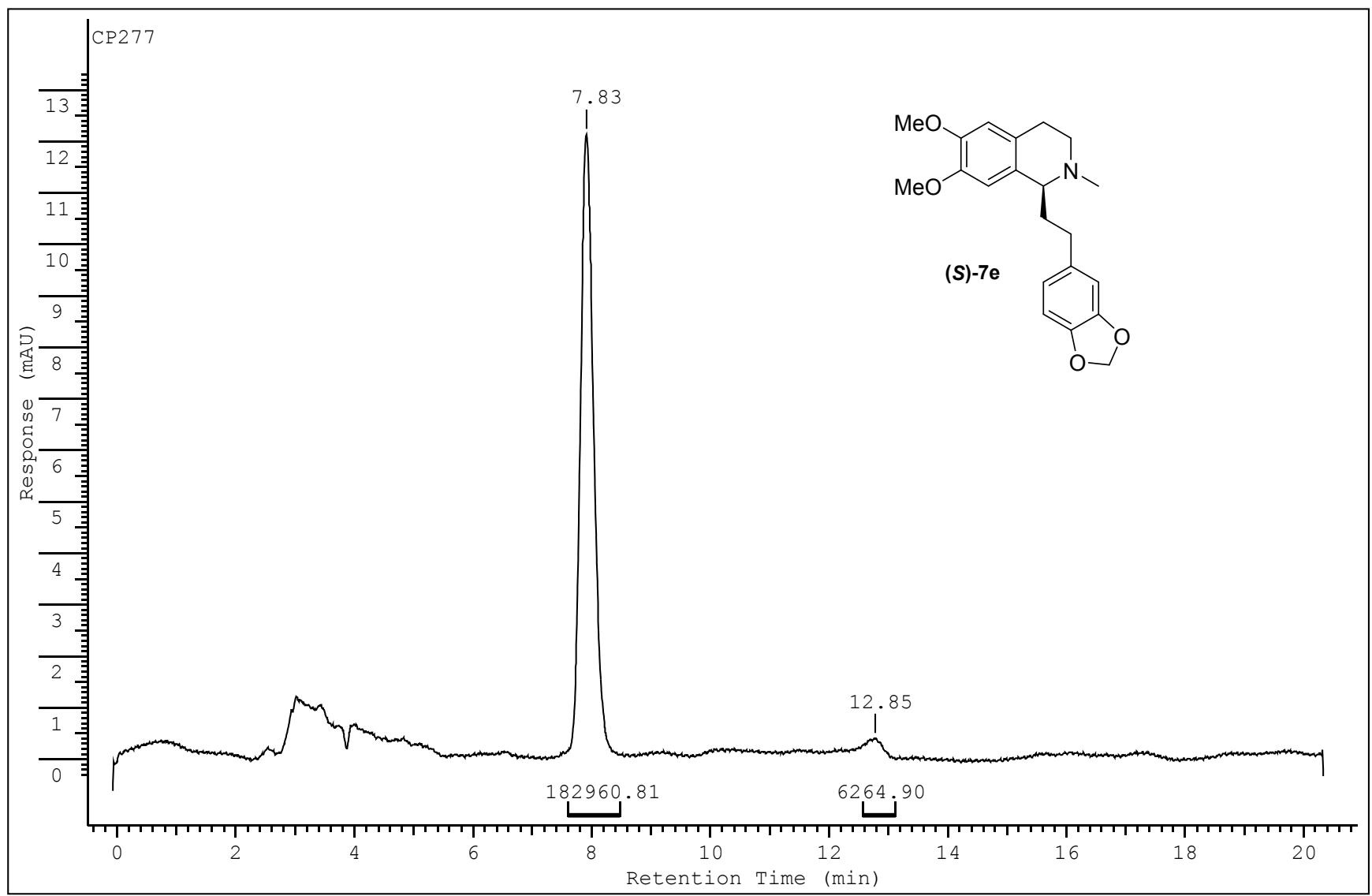


Figure S137. Chiral HPLC chromatogram of (S)-6,7-dimethoxy-1-(4-hydroxyphenethyl)- $N$-methyl-1,2,3,4-tetrahydroisoquinoline (S)-7f

\begin{tabular}{cccccccc} 
Sample & Peak & $\begin{array}{c}\text { RT } \\
{[\mathrm{min}]}\end{array}$ & $\begin{array}{c}\text { Peak Height } \\
{[\mathrm{mAU}]}\end{array}$ & $\begin{array}{c}\text { Integral } \\
{[\%]}\end{array}$ & $\begin{array}{c}\text { Peak Area } \\
{[\mathrm{mAU} \text { s }]}\end{array}$ & $\begin{array}{c}\text { FWHM } \\
{[\mathrm{min}]}\end{array}$ & $\begin{array}{c}\text { Integral Region } \\
{[\mathrm{min}]}\end{array}$ \\
\hline & 1 & 13.21 & 23.013 & 96.66 & 854786.94 & 0.865 & $12.64-15.07$ \\
CP309 & 2 & 32.23 & 0.953 & 3.34 & 29506.00 & 3.811 & $31.84-33.95$ \\
\hline
\end{tabular}

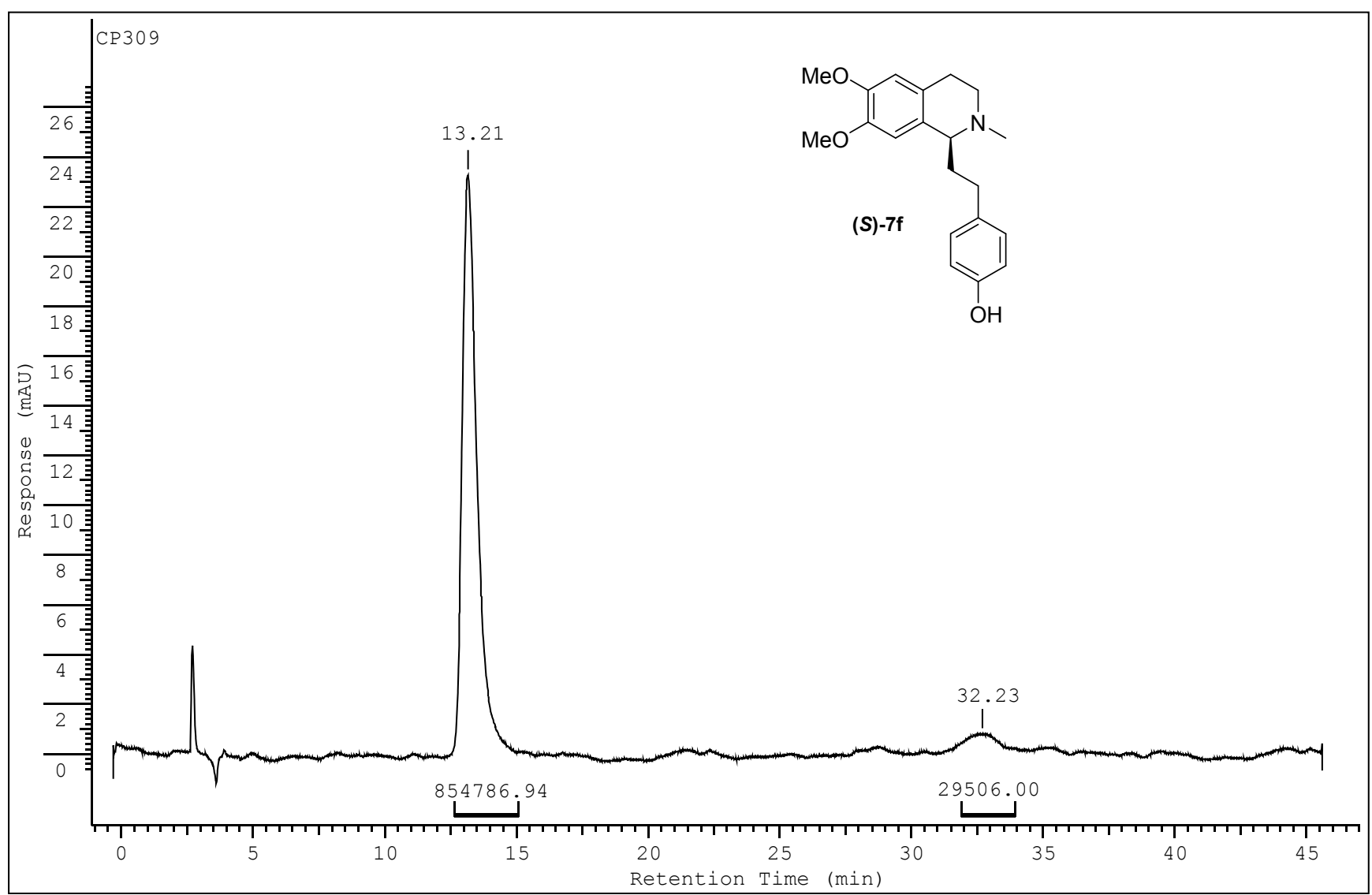


Figure S138. Chiral HPLC chromatogram of (S)-6,7-dimethoxy-1-(4-hydroxy-3-methoxyphenethyl)- $N$-methyl-1,2,3,4-tetrahydroisoquinoline (S)-7g

\begin{tabular}{|c|c|c|c|c|c|c|c|c|}
\hline Sample & Peak & $\begin{array}{c}\mathrm{RT} \\
{[\mathrm{min}]}\end{array}$ & $\begin{array}{c}\text { Peak Height } \\
{[\mathrm{mAU}]}\end{array}$ & $\begin{array}{c}\text { Integral } \\
{[\%]}\end{array}$ & $\begin{array}{c}\text { Peak Area } \\
{\left[\mathrm{mAU}^{*} \mathrm{~s}\right]}\end{array}$ & $\begin{array}{l}\text { FWHM } \\
{[\mathrm{min}]}\end{array}$ & $\begin{array}{c}\text { Integral Region } \\
\text { [min] }\end{array}$ & Anotation \\
\hline & 1 & 16.53 & 34.864 & 98.28 & 1712402.63 & 1.104 & $16.08-18.64$ & \\
\hline CP311 & 2 & 31.15 & 0.134 & 1.72 & 29912.46 & 1.152 & $31.42-34.19$ & \\
\hline
\end{tabular}

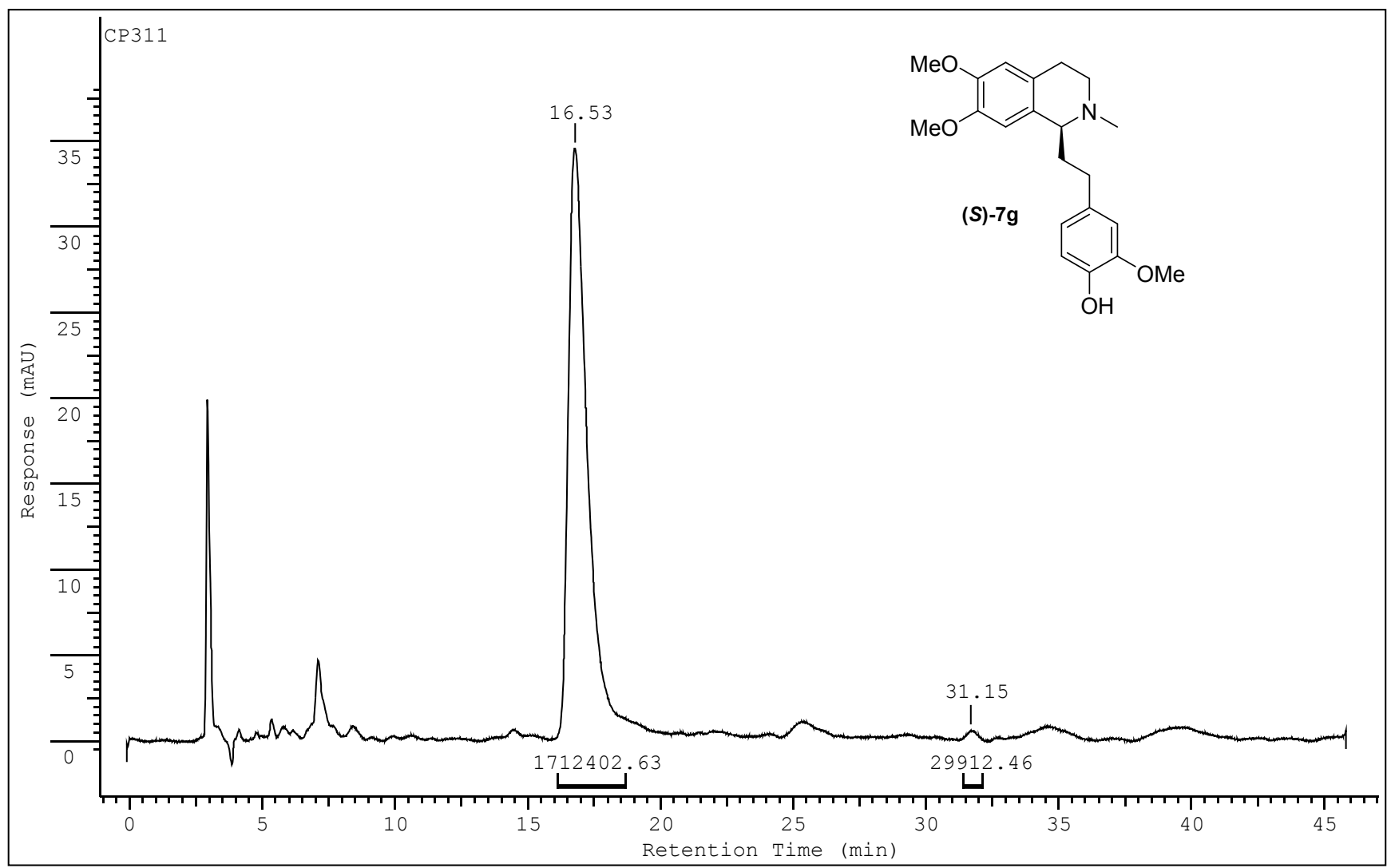


Figure S139. Chiral HPLC chromatogram of (S)-6,7-dimethoxy-1-(3-hydroxy-4-methoxyphenethyl)- $N$-methyl-1,2,3,4-tetrahydroisoquinoline (S)-7h

\begin{tabular}{cccccccc} 
Sample & Peak & $\begin{array}{c}\text { RT } \\
{[\mathrm{min}]}\end{array}$ & $\begin{array}{c}\text { Peak Height } \\
{[\mathrm{mAU}]}\end{array}$ & $\begin{array}{c}\text { Integral } \\
{[\%]}\end{array}$ & $\begin{array}{c}\text { Peak Area } \\
{[\mathrm{mAU} \text { * }]}\end{array}$ & $\begin{array}{c}\text { FWHM } \\
{[\mathrm{min}]}\end{array}$ & $\begin{array}{c}\text { Integral Region } \\
{[\mathrm{min}]}\end{array}$ \\
\hline & 1 & 16.67 & 71.574 & 94.62 & 3953349.25 & 1.253 & $16.37-19.38$ \\
CP307 & 2 & 26.48 & 8.036 & 5.38 & 224725.94 & 0.634 & $26.03-26.91$ \\
\hline
\end{tabular}

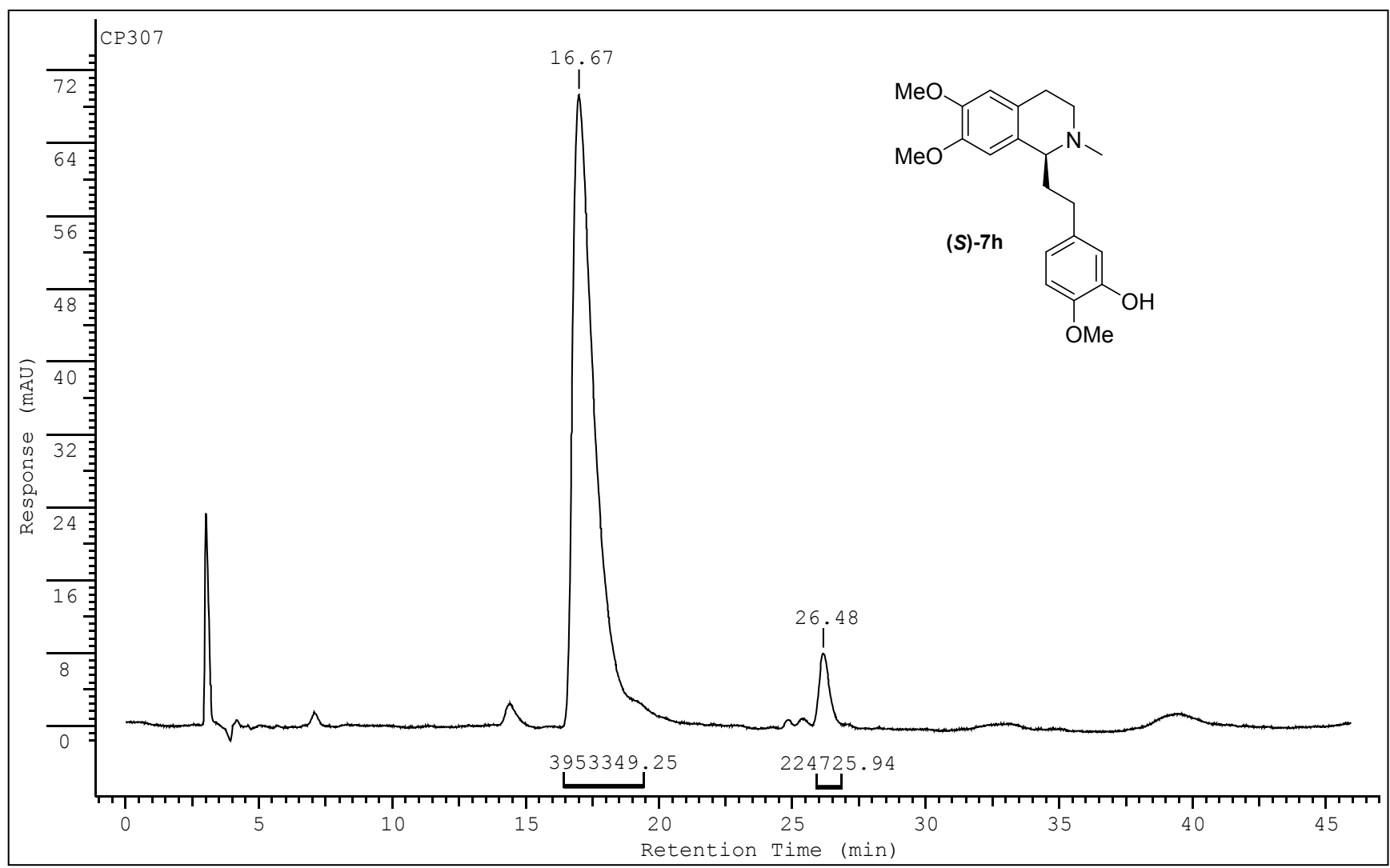


Figure S140. Chiral HPLC chromatogram of (S)-6,7-dimethoxy-1-(4-hydroxy-3,5-dimethoxyphenethyl)- $N$-methyl-1,2,3,4tetrahydroisoquinoline $(\boldsymbol{S})-\mathbf{- 7 i}$

\begin{tabular}{cccccccc} 
Sample & Peak & $\begin{array}{c}\text { RT } \\
{[\mathrm{min}]}\end{array}$ & $\begin{array}{c}\text { Peak Height } \\
{[\mathrm{mAU}]}\end{array}$ & $\begin{array}{c}\text { Integral } \\
{[\%]}\end{array}$ & $\begin{array}{c}\text { Peak Area } \\
{[\mathrm{mAU} \text { s }]}\end{array}$ & $\begin{array}{c}\text { FWHM } \\
{[\mathrm{min}]}\end{array}$ & $\begin{array}{c}\text { Integral Region } \\
{[\mathrm{min}]}\end{array}$ \\
\hline & 1 & 24.58 & 23.463 & 97.52 & 450107.25 & 1.809 & $24.12-27.10$ \\
CP308 & 2 & 36.19 & 1.779 & 2.48 & 11449.97 & 0.561 & $35.74-36.28$ \\
\hline
\end{tabular}

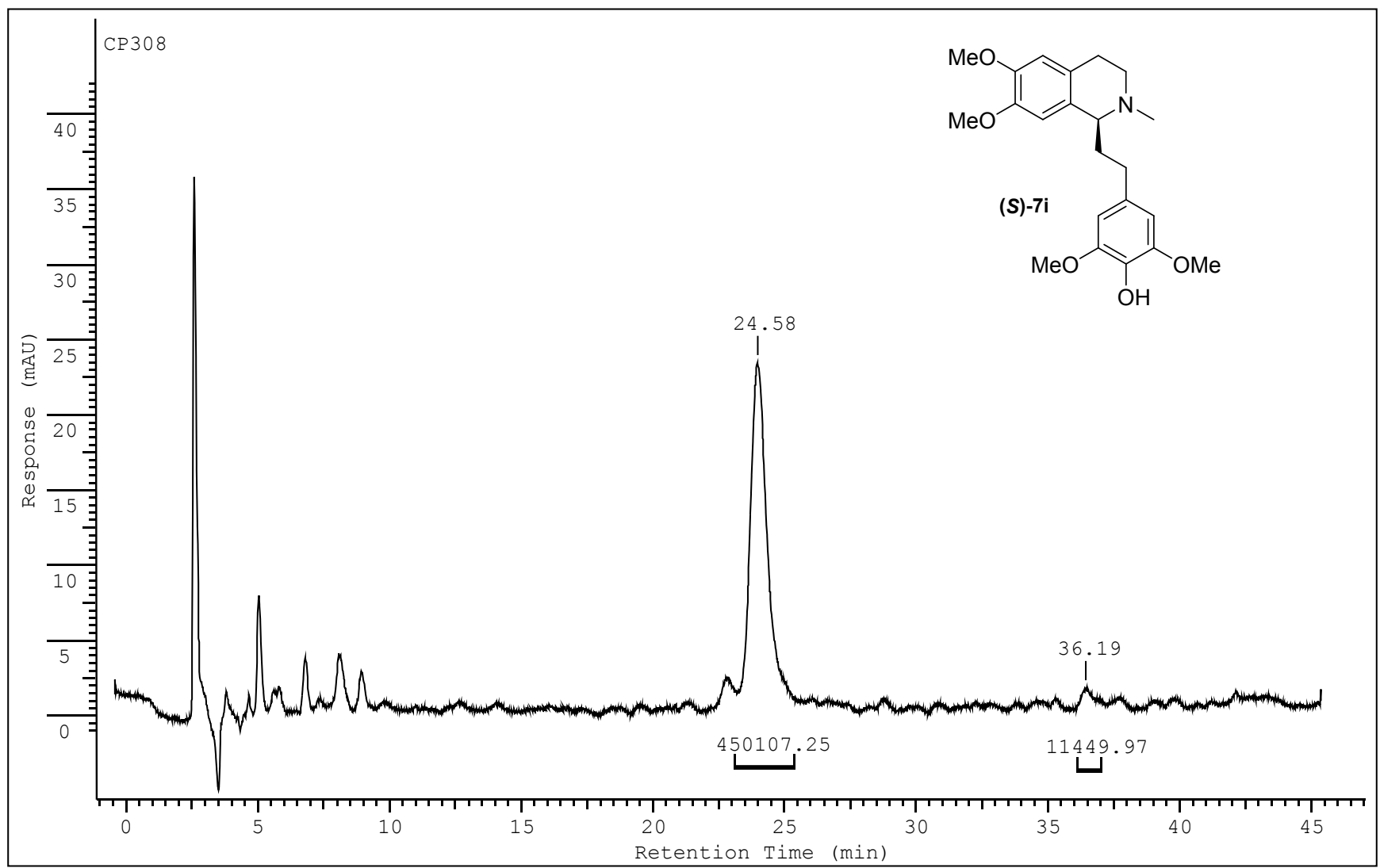

